\title{
Copyright
}

by

Raquel Yvonne Reynolds

2016 
The Dissertation Committee for Raquel Yvonne Reynolds Certifies that this is the approved version of the following dissertation:

\section{Prevalence and Characteristics of Prediabetes in Workers in Industry}

\section{Committee:}

Donna Lynn Rew, Supervisor

Tracie Harrison

Alexandra Garcia

Karen Johnson

Roberta Jeanne Ruiz 


\title{
Prevalence and Characteristics of Prediabetes in Workers in Industry
}

\section{by}

\section{Raquel Yvonne Reynolds, BSN; MSN}

\author{
Dissertation \\ Presented to the Faculty of the Graduate School of \\ The University of Texas at Austin \\ in Partial Fulfillment \\ of the Requirements \\ for the Degree of \\ Doctor of Philosophy
}

The University of Texas at Austin

May 2016 


\section{Dedication}

This dissertation is dedicated to my daughter, Marcella. She has been my cheerleader, my coach, my shoulder to cry on for six years. Her unfailing support has carried me through my journey. Thank you, Sweet Pea. It is also dedicated to my husband. He has been my anchor, keeping me steady and focused. Thank you, Jason. 


\section{Acknowledgements}

Thank you, Dr. Jerry Trzeciakowski for volunteering to help me with my statistics. You were amazing to work with and a great asset. 


\title{
Prevalence and Characteristics of Prediabetes in Workers in Industry
}

\author{
Raquel Yvonne Reynolds, PhD \\ The University of Texas at Austin, 2016
}

Supervisor: Lynn Rew

Type 2 diabetes is a chronic, progressive disease with serious health consequences. It affects an estimated 79 million (35\%) adults in the United States aged 20 years and older. Progression to Type 2 diabetes occurs slowly, with steady progression from normal glucose metabolism to impairment severe enough to indicate illness. Prediabetes is the interim period between normal glucose metabolism and impaired glucose metabolism, where glucose metabolism is abnormal but not yet serious enough to meet the diagnostic criteria for Type 2 diabetes. Without treatment or intervention, a person with prediabetes will develop Type 2 diabetes in 10 years. Risk for prediabetes and subsequent Type 2 diabetes is multifactorial. Empirical evidence has shown that risk factors include demographic, sociocultural, and health behavior variables. Therefore, the purposes of this dissertation study were to: (1) describe the prevalence of work stress (job strain and job imbalance) and prediabetes in full-time employees at the central Texas headquarters of a large, computer technology company (2) describe the relationship among stress, work stress, and prediabetes, (3) explore the potential interactive effects of socio-cultural factors, stress, work stress, health behaviors, and coping on prediabetes, and (4) explore the potential mediating effects of health behaviors and coping on the relationship between work stress and prediabetes. 
This study used a descriptive, cross-sectional design. It was conducted in a single, computer technology company in central Texas. A conceptual framework, The Conceptual Model for Metabolic Risk, was developed for this study. The concepts in this model included known risk factors for prediabetes and additional risk factors that were theorized. Well established instruments were used to measure: job roles, smoking, alcohol, diet, exercise, sleep, stress, work stress (job strain, job imbalance), and coping. Physiologic data were extracted from the participants' electronic medical record. The results indicated that prevalence of prediabetes was lower and the prevalence of work stress was higher in the participant sample than what is reported in the US population. Work stress and global stress were significantly correlated. Three variables predicted prediabetes: alcohol, job imbalance, and sleep. Job imbalance was inversely related to prediabetes $(\varrho=-0.192, p=0.022)$. The study findings suggested that job imbalance lowers risk of prediabetes $(\mathrm{OR}=0.05,95 \% \mathrm{CI}, 0.003-0.72, p=0.028)$. The inverse relationship between job imbalance and prediabetes contradicts previous empirical data. A mediation model was unsuccessful at finding a mediating relationship of health behaviors on the relationship between job imbalance and prediabetes.

Future prediabetes research should continue to explore the relationship between work stress and prediabetes and sleep and prediabetes. It would be especially important to include individual work-related characteristics, such as motivation, commitment, complacency, or passivity, to explore potential, underlying factors that may contribute to work stress. Individual work-related characteristics may explain the inverse relationship between job imbalance and prediabetes found in this study.

Patient assessment and education are the hallmarks of nursing practice. During prediabetes screening exams, nurses should include assessments of new risk factors (sleep, stress, and work stress) with assessments of traditional risk factors (age, BMI). 
Patient education aimed at reducing the risk of prediabetes should include information about coping with stress and maintaining healthy sleep habits. Work-site education programs should include programs on work-life balance, coping with stress, and the significance of sleep. 


\section{Table of Contents}

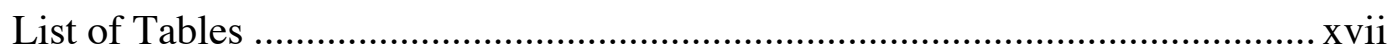

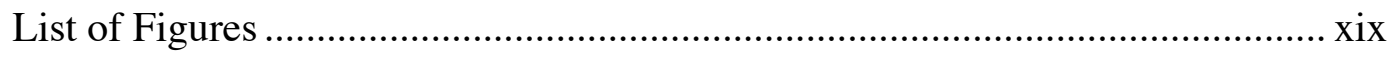

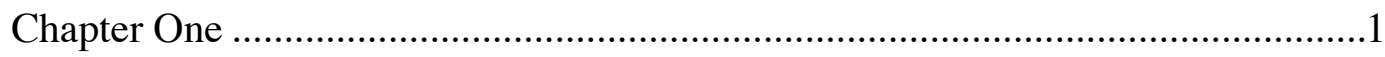

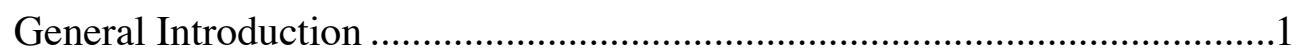

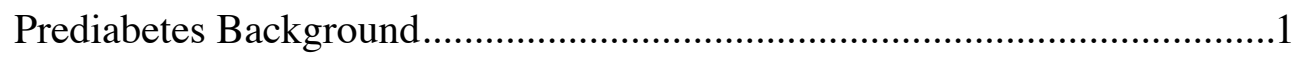

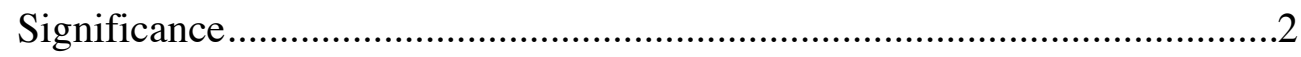

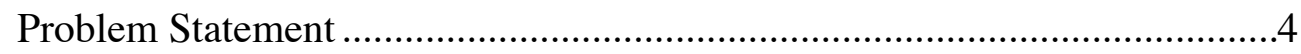

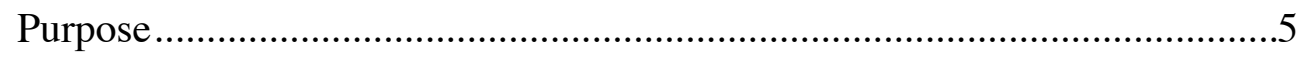

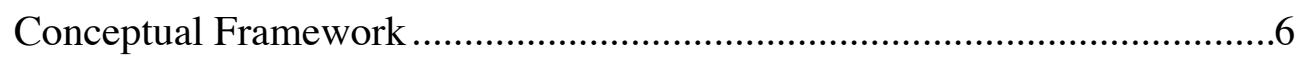

The Conceptual Model for Metabolic Risk …………………………..........

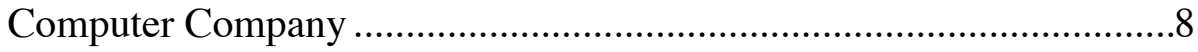

Socio-cultural Factors ………………………………………….....

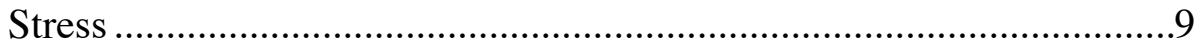

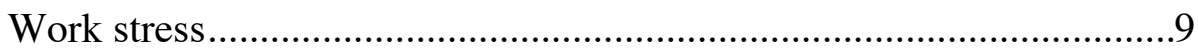

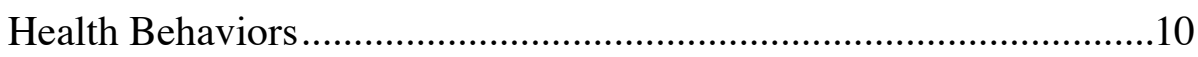

Metabolic Profile ………………………….......................................11

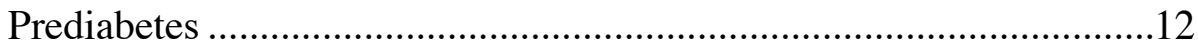

Concepts and Proposed Relationships ………………..............................12

Socio-Cultural Factors .....................................................................13

Socio-Cultural Factors and Work Stress ......................................13

Socio-Cultural Factors and Health Behaviors..............................13

Socio-Cultural Factors and Prediabetes within the Metabolic Profile . .14

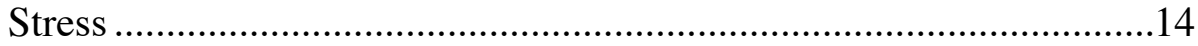

Stress and Work Stress.........................................................15

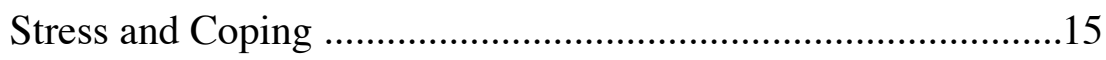

Work Stress and Prediabetes within the Metabolic Profile ........16

Work Stress and Health Behaviors .............................................16 


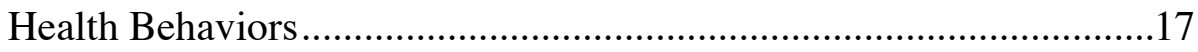

Health Behaviors and Work Stress ............................................17

Health Behaviors and Prediabetes within the Metabolic Profile 17

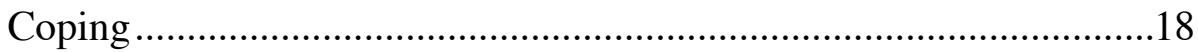

Coping and Prediabetes within the Metabolic Profile ................18

Prediabetes within the Metabolic Profile .......................................18

Conceptual and Operational Definitions........................................................19

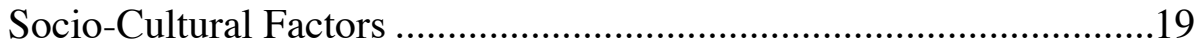

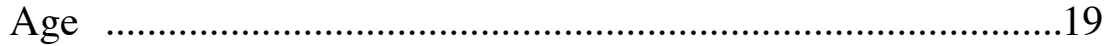

Sex

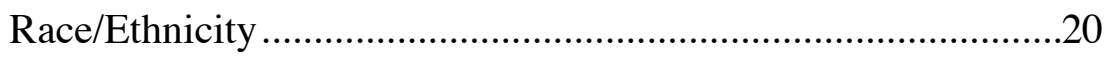

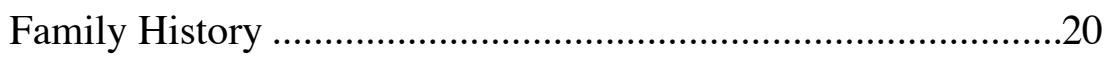

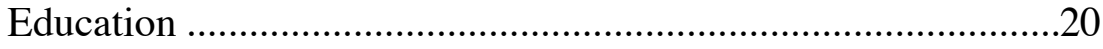

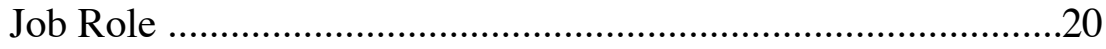

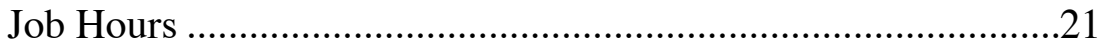

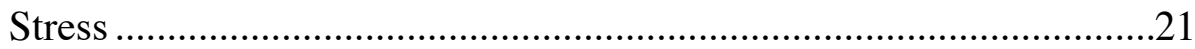

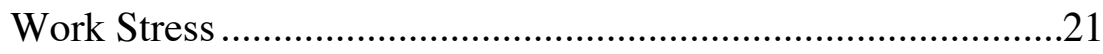

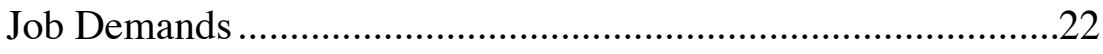

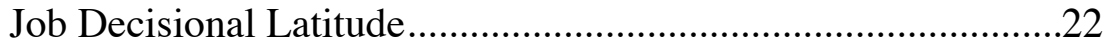

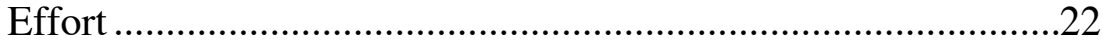

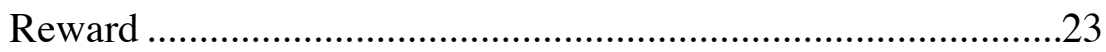

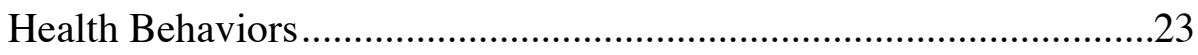

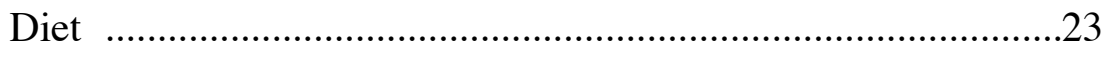

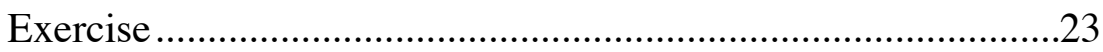

Sleep

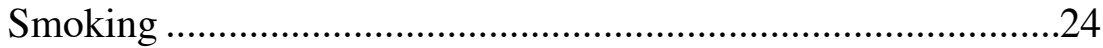

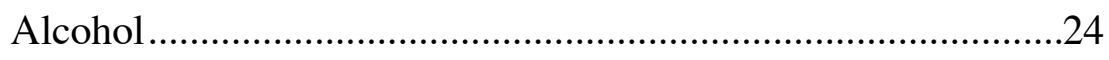

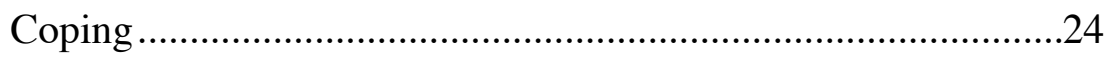

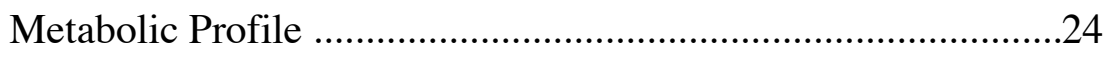

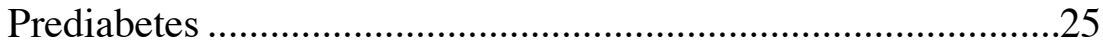




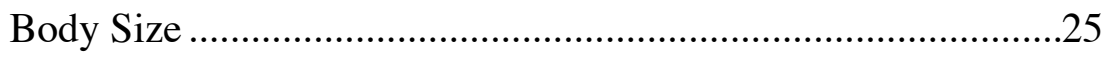

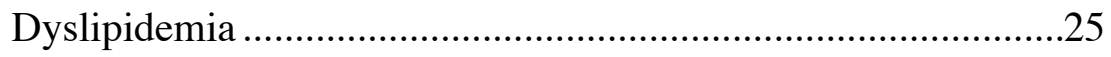

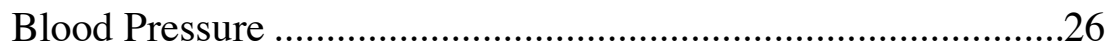

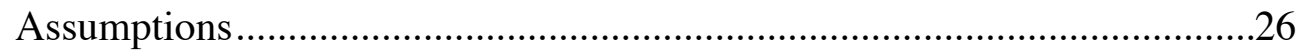

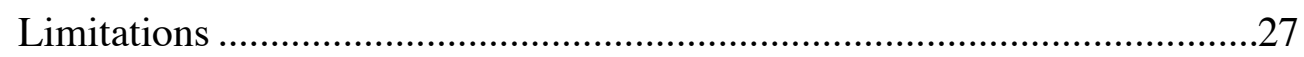

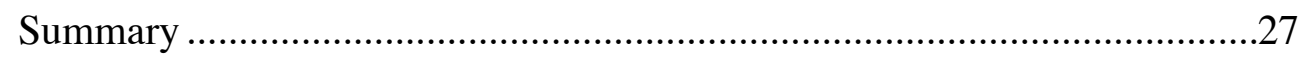

Chapter Two

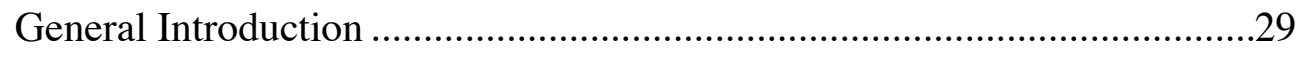

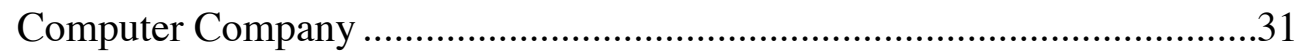

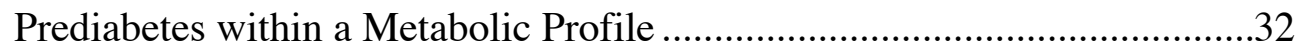

Body Size: Waist Circumference and Body Mass Index ..................................34

Dyslipidemia: HDL and Triglycerides.........................................................37

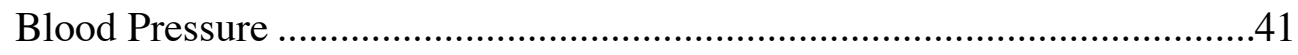

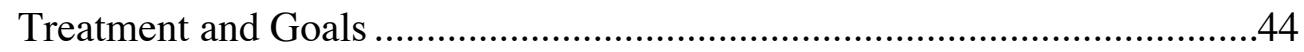

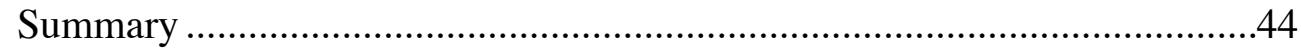

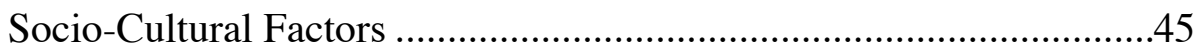

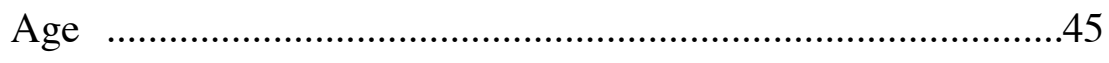

Sex

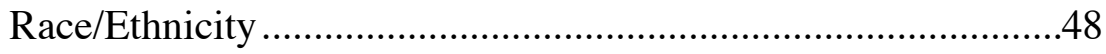

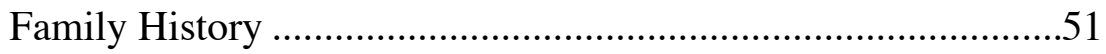

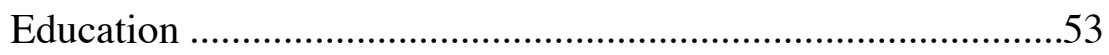

Job Characteristics: Role and Hours ……………………….......55

Summary of Socio-Cultural Factors ……………………………........

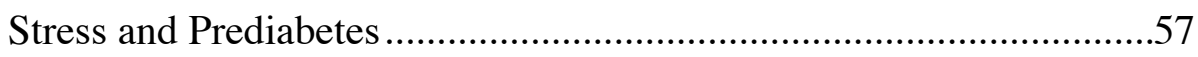

Summary of Stress and Prediabetes ........................................................60

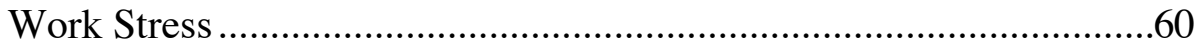

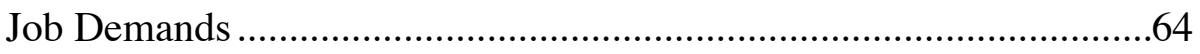

Decisional Latitude .............................................................................

Interaction Between Job Demands and Decisional Latitude .........................75

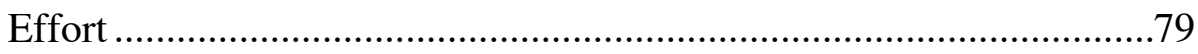




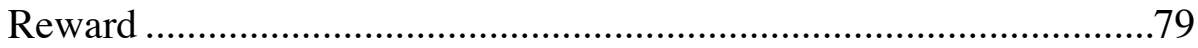

Interaction between Effort and Reward: Job Imbalance ...............................79

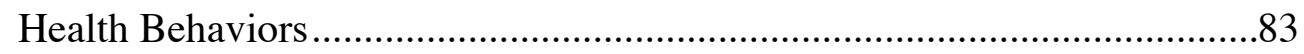

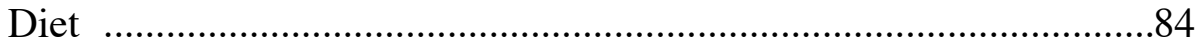

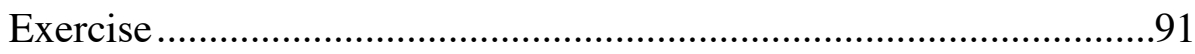

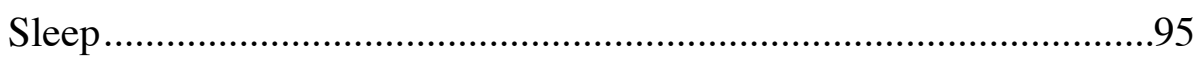

Smoking and Alcohol .......................................................................100

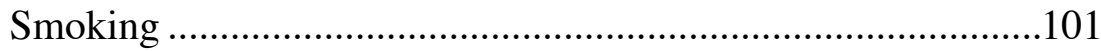

Alcohol ..........................................................................102

Summary of Health Behaviors ...................................................................106

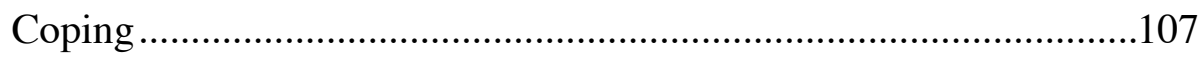

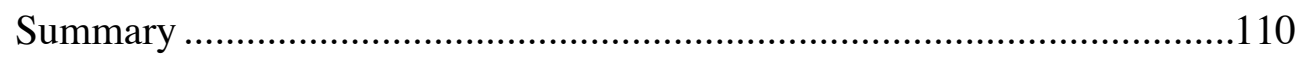

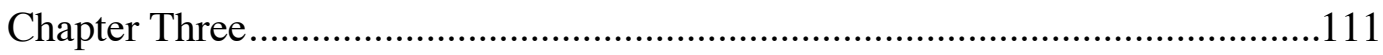

Methods and Research Design ...............................................................111

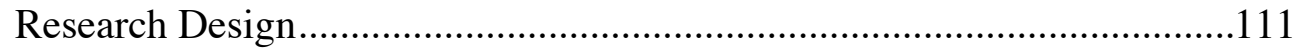

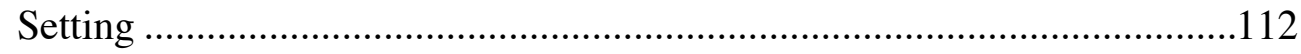

Sample

Inclusion Criteria …………………………………................113

Exclusion Criteria …………………………………………….........

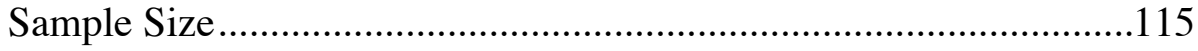

Human Subject Protection ………………………………………...115

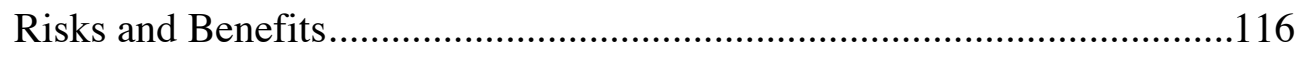

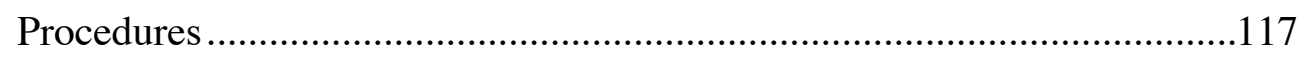

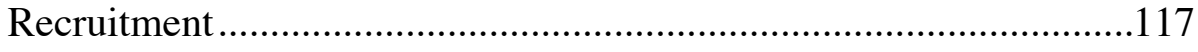

Consent and Enrollment.......................................................................

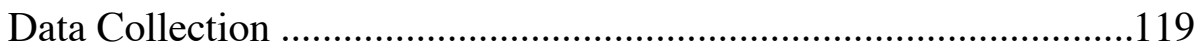

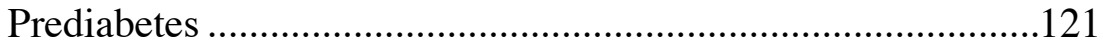

Metabolic Profile ………………………………………...122

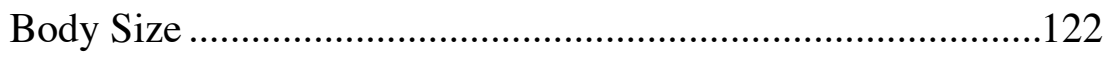

Body Mass ………………………………………………....122

xii 


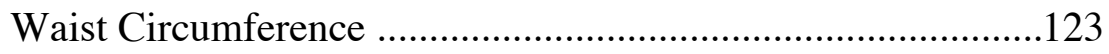

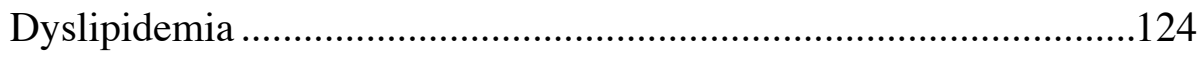

High-Density Lipids................................................................124

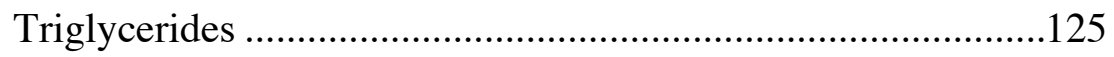

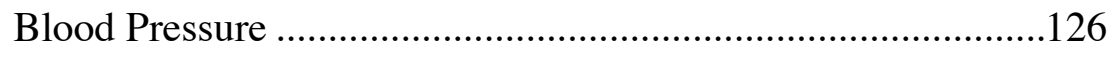

Socio-cultural Aspects ...............................................................127

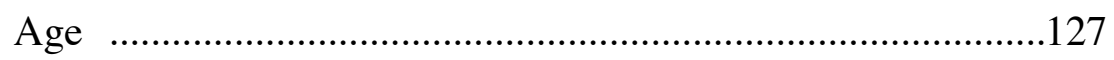

Sex

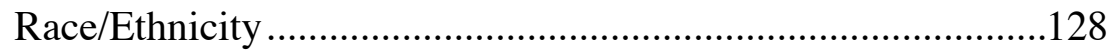

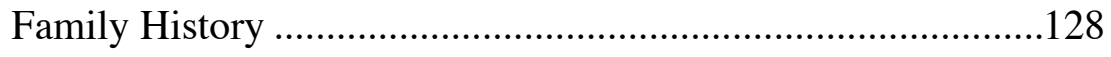

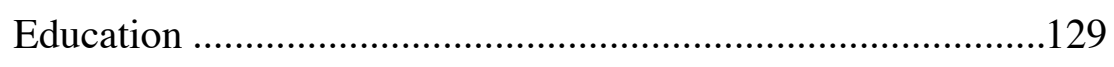

Job Role and Job Hours .......................................................130

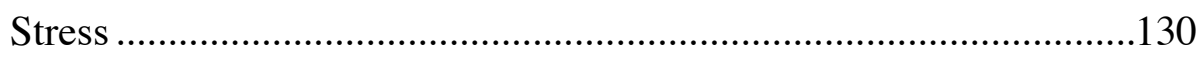

Perceived Stress Scale...........................................................131

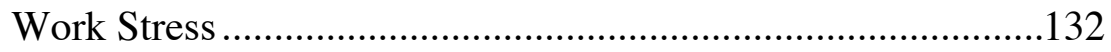

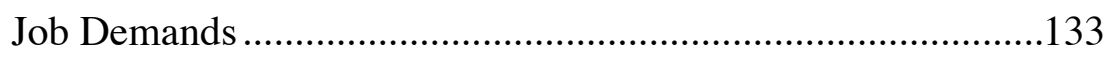

Decisional Latitude ....................................................................

Job Content Questionnaire ...........................................................134

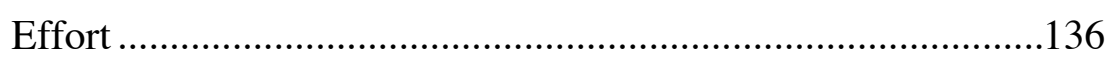

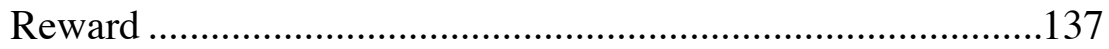

Effort Reward Imbalance Questionnaire ……………………...137

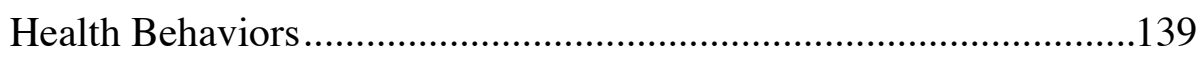

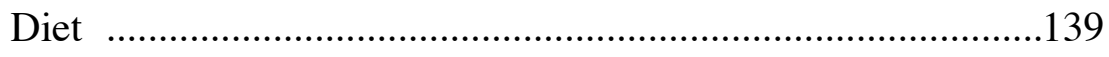

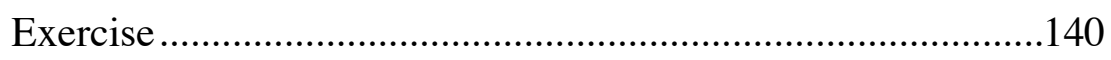

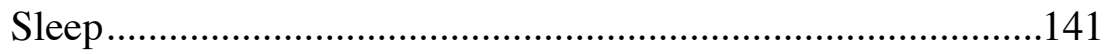

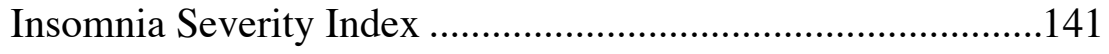

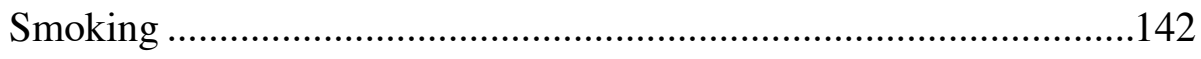

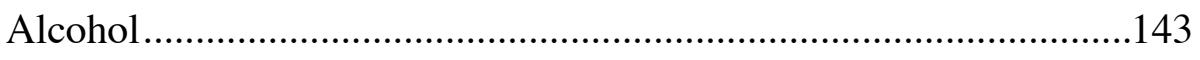

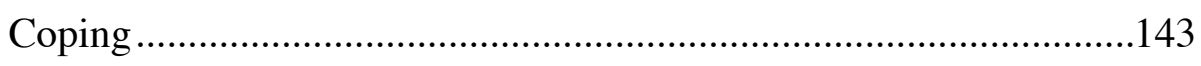

Brief Approach/Avoidance Coping Questionnaire ....................143 


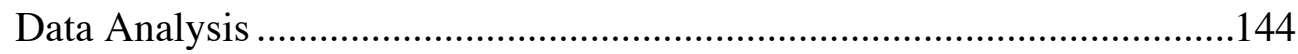

Data Management and Cleaning ........................................................144

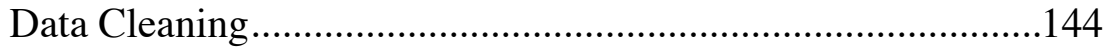

Research question 1: What is the prevalence of prediabetes in full-time

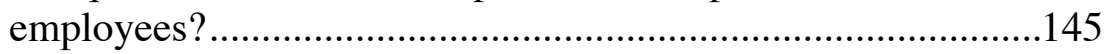

Research question number 2: What is the prevalence of job strain in fulltime employees?..................................................................146

Research question number 3: What is the prevalence of job imbalance in full-time employees? ...............................................................146

Research question number 4: What is the relationship between job strain and prediabetes in full-time employees?...................................147

Research question number 5: What is the relationship between job imbalance and prediabetes in full-time employees? ..................147

Research question number 6: What is the relationship between job strain

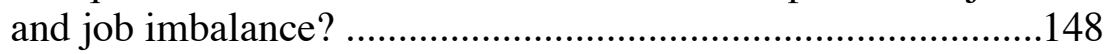

Research question number 7: What is the relationship between job strain and stress? ...................................................................148

Research question number 8: What is the relationship between job imbalance and stress?...............................................................

Research question number 9: What combination of independent variables (socio-cultural factors, health behaviors, work stress) best predicts prediabetes?.......................................................................149

Research question number 10: Do health behaviors mediate the relationship between work stress and prediabetes?

Research question number 11: Does coping mediate the relationship between either job strain or job imbalance and prediabetes? ...152

Summary .153

Chapter Four 155

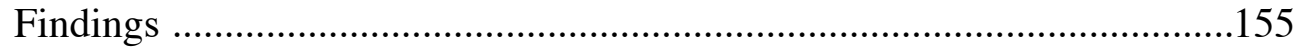

Demographic Characteristics of Study Participants ………………...155

Descriptive Statistics for the Study Variables ..........................................158

Metabolic Profile Variables ..............................................................158

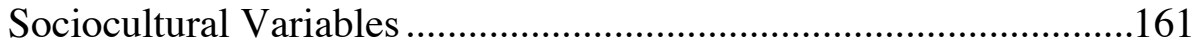




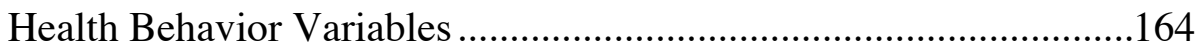

Findings from Data Analyses by Research Question .................................168

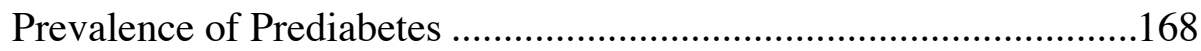

Prevalence of Job Strain..................................................................170

Prevalence of Job Imbalance ……………………………………......170

Relationship Between Job Strain and Job Imbalance ........................171

Relationship Between Job Strain and Prediabetes ..............................172

Relationship Between Job Imbalance and Prediabetes .......................173

Relationship Between Job Strain and Stress ......................................174

Relationship Between Job Imbalance and Stress ...............................175

Predictors of Prediabetes.................................................................176

Mediation of Relationship Between Work Stress and Prediabetes....180 Coping as a Mediator ………………………………………......183

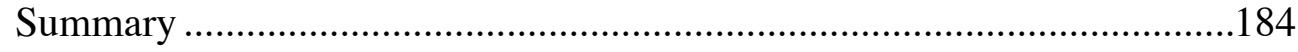

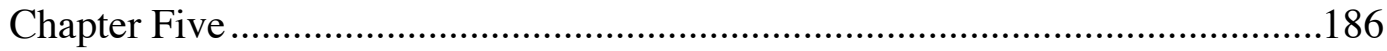

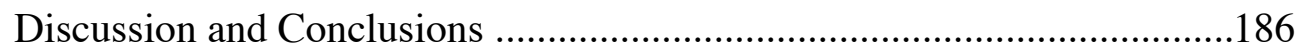

Discussion of the Findings ..............................................................186

Prevalence of Job Strain...........................................................187

Prevalence of Job Imbalance ...................................................190

Job Strain and Job Imbalance ……………………………........191

Job Strain and Prediabetes ........................................................193

Job Imbalance and Prediabetes ....................................................194

Job Strain and Stress .............................................................195

Job Imbalance and Stress ........................................................197

Predictors of Prediabetes.............................................................200

Health Behaviors as Mediators .................................................203

JS=Job Strain; JI=Job Imbalance; PSS=Perceived Stress Scale .........203

Conceptual Model for Metabolic Risk ..............................................204

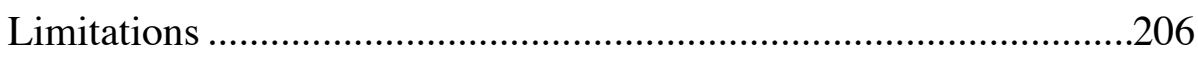

Implications and Recommendations ........................................................208 


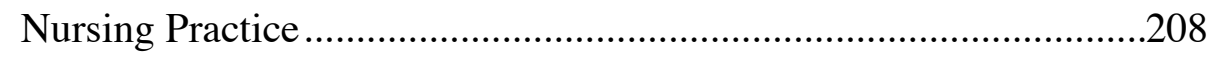

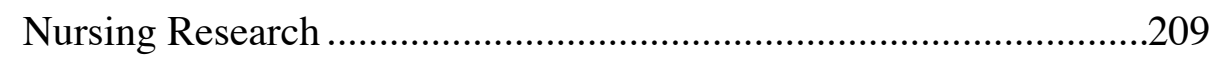

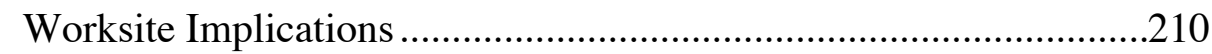

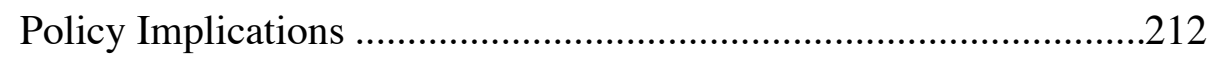

Recommendations for Study Site...................................................213

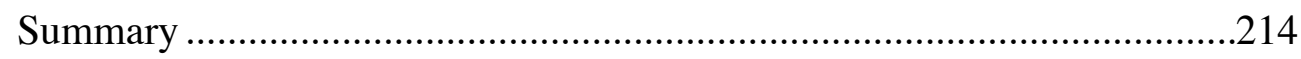

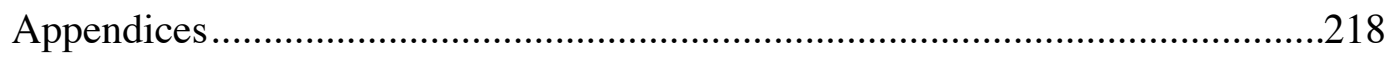

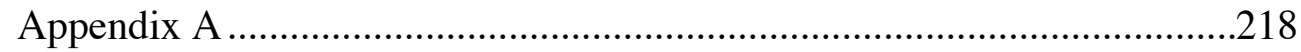

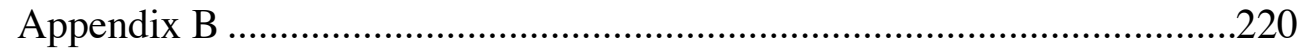

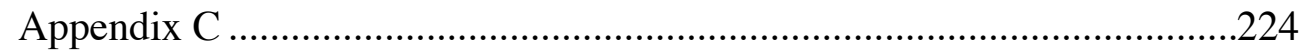

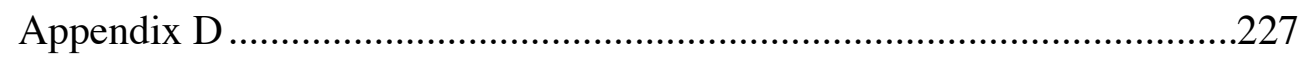

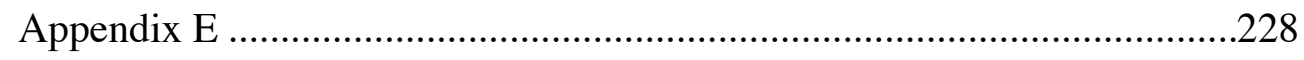

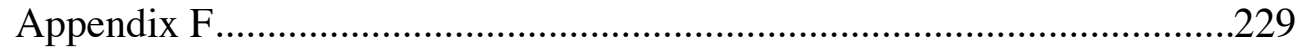

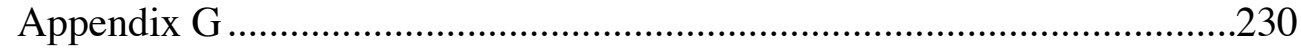

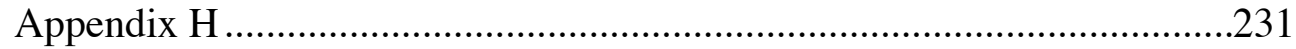

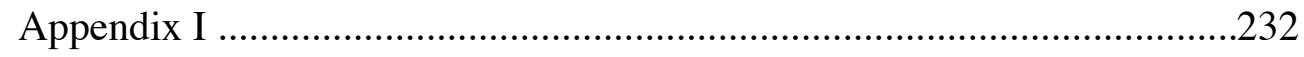

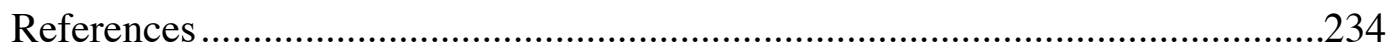

Vita 


\section{List of Tables}

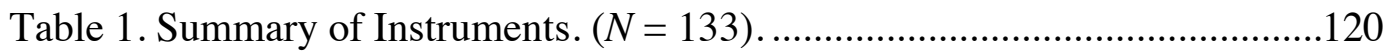

Table 2. Concepts within the Conceptual Model for Metabolic Risk.................153

Table 3. Demographic Characteristics of Participants.....................................156

Table 4. Demographic Characteristics of Participants....................................157

Table 5. Summary of Metabolic Variables.....................................................159

Table 6. Summary of Sociocultural Variables...............................................162

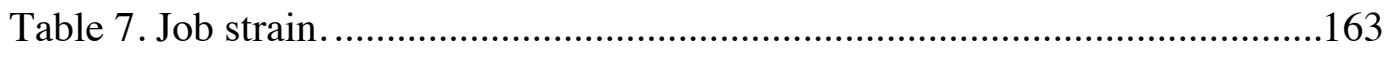

Table 8. Summary of Health Behavior Variables.............................................165

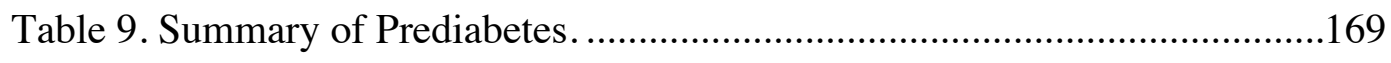

Table 10. Summary of Job Strain. .............................................................170

Table 11. Summary of Job Imbalance. ......................................................171

Table 12. Summary of Spearman's rho correlation for job strain and job imbalance. 172

Table 13. Summary of Spearman's rho correlation for job strain and prediabetes.173

Table 14. Summary of Spearman's rho correlation for job imbalance and prediabetes.

Table 15. Summary of Spearman's rho correlation for job strain and global and eventspecific stress. 175

Table 16. Summary of Spearman's rho correlation for job imbalance and global and event-specific stress. ..........................................................176

Table 17. Spearman's rho bivariate correlations. ...........................................178

Table 18. Predictors Selected as Candidates for Main Effects...........................179

Table 19. Goodness of Fit Indices for SEM Model........................................182 
Table 20. Summary of Study Findings ... 


\section{List of Figures}

Figure 1. Conceptual Model for Metabolic Risk..............................................

Figure 1. Conceptual Model for Metabolic Risk ............................................29

Figure 2. The hypothesized model of mediation. .........................................183 


\section{Chapter One}

\section{GENERAL INTRODUCTION}

Prediabetes is a chronic, progressive condition. The condition marks the state of health between normal glucose metabolism and Type 2 diabetes mellitus (T2DM). During prediabetes, symptoms, such as fatigue and increased appetite, range from subtle to unnoticeable. The risk factors for prediabetes are multidimensional. The primary physiologic sign of prediabetes is high blood glucose. However, evidence shows that other metabolic risk factors may be present, such as large body size, dyslipidemia, and high blood pressure (American Diabetes Association [ADA], 2013a). A socio-cultural, genetic risk factor that increases the risk for prediabetes is family history. Other sociocultural factors, such as age, sex, and race/ethnicity, may be associated prediabetes. Evidence has shown that stress affects the onset of diabetes through activation of stress hormones that increase blood glucose (Lloyd, Smith \& Weinger, 2005). Work stress is a type of stress; repeated and chronic work stress activates the stress response (World Health Organization [WHO], 2007). Therefore, work stress is a potential risk factor for prediabetes. Health behaviors that affect glucose metabolism and, in turn, affect

prediabetes are sedentary lifestyle, unhealthy diet, poor sleep (ADA, 2013c), smoking (United States Health and Human Services [USHHS], 2014), and overuse of alcohol (Baik \& Shin, 2008). If left untreated, prediabetes often leads to T2DM (ADA, 2013a).

\section{Prediabetes Background}

In 1997 and 2003, The Expert Committee on Diagnosis and Classification of Diabetes Mellitus identified an intermediary condition in which individuals had glucose levels that were higher than normal but not yet high enough to meet the diagnostic criteria for T2DM (ADA, 2010). Individuals in this intermediary group were found to 
have metabolic abnormalities in carbohydrate, fat, and protein metabolism, and, consequently, had impaired fasting glucose or impaired glucose tolerance. This interim period when unhealthy, metabolic changes that lead to T2DM occur is called prediabetes (ADA, 2013a). The term prediabetes has replaced former names that are no longer used: borderline diabetes, subclinical diabetes, and chemical diabetes (ADA, 2013b).

Prediabetes is a consequence of physiologic, metabolic changes in glucose metabolism and utilization. Two processes occur during prediabetes, impaired fasting glucose (IFG) or impaired glucose tolerance (IGT). The Expert Committee on Diagnosis and Classification of Diabetes Mellitus adopted reference levels in for IFG (ADA, 2003) and IGT (ADA, 2010) to maximize sensitivity and specificity in detecting future risk of T2DM. Impaired fasting glucose is indicated when fasting glucose levels are above normal but not yet high enough to indicate T2DM (100-126mg/dL). Likewise, impaired glucose tolerance is indicated when 2-hour oral glucose tolerance tests $(140-199 \mathrm{mg} / \mathrm{dL})$ or glycated hemoglobin (5.7-6.4\%) are abnormal but do not meet the diagnostic criteria for T2DM. Only one abnormal test is necessary to confirm prediabetes. Abnormalities in IFG and IGT are associated with metabolic conditions such as obesity, dyslipidemia, and hypertension. As such, prediabetes is within a cluster of metabolic conditions. Prediabetes is associated with a relatively high risk for future development of T2DM and cardiovascular disease (ADA, 2010).

\section{SIGNIFICANCE}

An estimated 79 million (35\%) adults in the United States (US) aged 20 years and older were diagnosed with prediabetes in 2010. After adjusting for age and gender differences, 35\% of non-Hispanic Blacks, 35\% of non-Hispanic Whites, and 36\% of Hispanic adults in the US were diagnosed with prediabetes in 2010 (CDC, 2011). 
Estimated medical cost of prediabetes is \$25 billion annually through inpatient and ambulatory care visits (Zhang et al., 2009). Each year, persons with untreated prediabetes have a 6-10\% increase in risk for T2DM (DPP, 2002). Type 2 diabetes mellitus is a serious, chronic, progressive illness. Once diagnosis for T2DM is made, the damage sustained by pancreatic beta cells is irreversible. Upon T2DM diagnosis, up to $80 \%$ of pancreatic beta cell function has already been lost (Abdul-Ghani \& DeFronzo, 2009; Rao et al., 2004). Type 2 diabetes mellitus has no cure. Without proper treatment and management, prolonged T2DM can lead to blindness, lower limb amputation, soft tissue infection, kidney failure, and early mortality (ADA, 2013). Natural history and pathogenesis research indicate that the prediabetes period is prolonged. Progression to T2DM can take up to 10 years (Diabetes Prevention Program Research Group [DPP], 2010; Rao, Disraelli \& McGregor, 2004). The extended time period between prediabetes and T2DM provides an opportunity for early identification of prediabetes and the related metabolic risks.

The ADA recommends screening for prediabetes in adults aged 45 and older and in younger adults with metabolic risk factors such as large body size, dyslipidemia, or high blood pressure. The median age for a US worker is 41.9 with projected increase to 42.6 by the year 2020 (US Bureau of Labor Statistics [BLS], 2014). Workers represent half of the world's population (WHO, 2007a). Workers spend most of their waking hours at work. The average US worker spends 8.8 hours per day at work (BLS, 2013). Up to $40 \%$ of US workers report experiencing chronic stress at work (WHO, 2007), and onefourth of US workers view their job as their number one life stressor (NIOSH, 2013). Chronic stress has been linked to impaired glucose and T2DM (Surwit \& Schnieder, 1992; Surwit \& Williams, 1996), especially in persons with other risk factors for T2DM (Lloyd, Smith \& Weinger, 2005). Work stress, a type of stress, stimulates the stress 
response and leads to psychological or physical illness (EFILWC, 2007; Schnall, Dobson \& Rosskam, 2009; WHO, 2007).

The Diabetes Prevention Program lifestyle intervention demonstrated that modest changes in health behaviors, such as weight loss and exercise, resulted in a $58 \%$ reduction in risk for developing T2DM (DPP, 2002; DPP, 2009). Worksite wellness programs have proven to be beneficial in reducing metabolic risk factors such as obesity and high blood pressure (Carthenon et al., 2009; McCraty, Atkinson \& Tomasino, 2003). Early identification of prediabetes in the workplace will provide an opportunity for developing worksite health education, counseling, and support interventions designed for early identification of prediabetes and its related risk factors. The individual benefit of early identification is to delay or prevent progression to T2DM. Understanding the worksite trends for prediabetes and its contributing and precipitating risk factors may help worksites to develop, modify, or change workplace practices to promote health and reduce risk of prediabetes.

\section{Problem Statement}

Prediabetes is a multifactorial condition. The symptoms of prediabetes are subtle and often go unnoticed. However, despite the subtlety of symptoms, there are underlying changes in glucose metabolism, such as insulin resistance at the receptor sites, insulin secretory defect by the pancreas, or both (Abdul-Ghani \& DeFronzo, 2009; Rao et al., 2004). When prediabetes is confirmed, there is already up to a $50 \%$ loss of pancreatic beta cell function. With no intervention, the pancreatic beta cell loss and the impairment in glucose metabolism will progress over time. In addition, there is evidence that there are multiple risk factors that affect (e.g., age) or are concurrent (e.g., dyslipidemia) with prediabetes. A potential risk factor for prediabetes is work stress. Work stress has been 
linked to an increase in the stress response and related physiological consequences (e.g., metabolic changes) (EFILWC, 2007), but the relationship between work stress and prediabetes has not been fully explicated. The contributing effects of prediabetes risk factors and their interactive effects on prediabetes and work stress have not been fully explored.

\section{Purpose}

The purposes of this study are to describe the prevalences of prediabetes (dependent variable) and work stress (job strain and job imbalance) in employees at a large computer technology company within the Professional, Scientific, and Technical Services sector headquartered in central TX; to describe the relationship between stress, work stress, and prediabetes; to explore the potential interactive effects of socio-cultural factors, stress, work stress, health behaviors, and coping on prediabetes; and to explore the potential mediating effects of health behaviors and coping on the relationship between work stress and prediabetes. As such, the research questions are as follows:

1. What is the prevalence of prediabetes in full-time employees?

2. What is the prevalence of job strain in full-time employees?

3. What is the prevalence of job imbalance in full-time employees?

4. What is the relationship between job strain and prediabetes in full-time employees?

5. What is the relationship between job imbalance and prediabetes in full-time employees?

6. What is the relationship between job strain and job imbalance?

7. What is the relationship between job strain and stress?

8. What is the relationship between job imbalance and stress? 
9. What combination of independent variables (socio-cultural factors, health behaviors, work stress) best predicts prediabetes?

10. Do health behaviors mediate the relationship between work stress and prediabetes?

11. Does coping mediate the relationship between either job strain or job imbalance and prediabetes?

\section{CONCEPTUAL FRAMEWORK}

A conceptual model was developed for this study after extensive review of screening and diagnostic criteria, treatment guidelines, and empirical data related to prediabetes, the outcome variable. Unless otherwise stated, screening, diagnostic, and treatment guidelines were based on statements from the American Diabetes Association (ADA), the American College of Endocrinology (ACE), the American College of Clinical Endocrinology (ACCE), or the National Institute of Diabetes and Digestive and Kidney Diseases (NIDDK). Empirical data is largely based on original research, although systematic reviews, meta-analyses, and epidemiological data were also included. The model, Conceptual Model for Metabolic Risk, is situated within a contextual environment, employment at computer company, the setting for this study. The sociocultural factors were derived from screening, diagnostic, and empirical data. The role of stress in glucose metabolism and risk for T2DM has extensive, empirical support. A component of stress, work stress, has limited empirical support for its relationship with glucose metabolism and T2DM, but extensive support for its relationship with metabolic disease. The concepts related to health behaviors were based on empirical evidence that suggest a direct or mediating relationship with prediabetes. Coping was added due to the long established relationship with stress and the potential mediating effect on health 
derived from empirical support. The Conceptual Model for Metabolic Risk positions prediabetes within the metabolic profile, which includes a cluster of metabolic risk factors that often occur with prediabetes. The metabolic profile was drawn from expert agency recommendations for prediabetes screening, diagnostics, and treatment. The combined effects of the metabolic profile lead to serious consequences, T2DM and cardiovascular disease. These long-term effects of the metabolic profile were beyond the scope of this study. The synthesis of expert agency screening recommendations and empirical evidence determined the proposed relationships depicted in the model (see Figure 1).

Figure 1. Conceptual Model for Metabolic Risk.

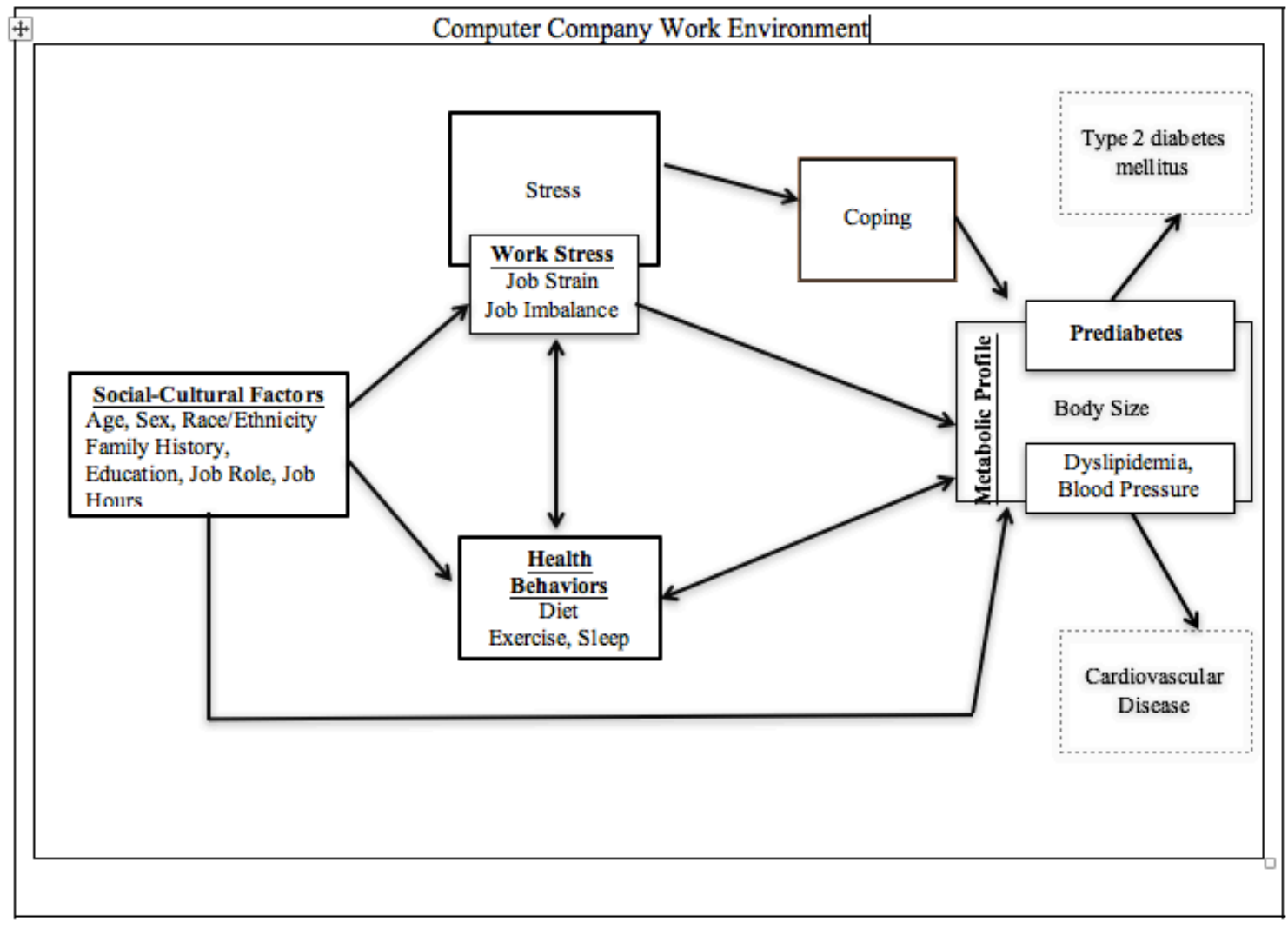




\section{The Conceptual Model For Metabolic RiSK}

\section{Computer Company}

Since the mid-1960s, studies that have shown relationships between work stress or work situations and metabolic illness have been largely set in industrial or technical settings (Marmot et al., 1978). The central Texas computer company provides a similar and convenient setting for this exploratory study. The company selected is large, computer technology company headquartered in central Texas that manufactures test and measurement hardware and software systems. There are approximately 2,400 employees in central Texas with a variety of job roles including office/clerical, graphic design, engineering, information technology, manufacturing, marketing, web design, sales and services.

\section{Socio-cultural Factors}

The socio-cultural factors included in this study were: age, sex, race/ethnicity, family history, education, job role, and job hours. These socio-cultural factors were derived from screening, diagnostic, and treatment recommendations outlined by expert agencies. The ADA, ACE/ACCE, and ACE recommend screening individuals for prediabetes if aged 45 or older, ethnic minority, or have a family history of T2DM in at least one first-degree relative (ADA, 2013; Bloomgarden, 2008a; Garber et al., 2008). Screening recommendations and empirical data for sex are mixed, with some suggesting males at higher risk while others suggest females are at higher risk for prediabetes. Empirical evidence has long associated education with health outcomes, including T2DM. Empirical evidence has shown that education has a negative association with

poor health outcomes (Cutler \& Lleras-Muney, 2007; Fonseca \& Zheng, 2011; Landsbergis et al., 2013); each additional year beyond high school is associated with a 
$2.8 \%$ reduction of reporting poor health (Fonseca \& Zheng, 2011). Job role and job hours and their association with health outcomes also have strong empirical support. Lower status job roles (Karasek et al., 1981; Karasek et al., 1987; Karasek \& Theorell, 1990; Marmot, et al., 1978), night shift (Biggi et al., 2008), alternating shift (deBacquer et al., 2009; Karlsson et al., 2003; Suwazono, 2009) or overtime (Taris et al., 2011) job hours have been shown to have a direct relationship with health outcomes.

\section{Stress}

Acute and chronic stress activate the hypothalamic-pituitary-adrenocortical (HPA) axis. The activation of the HPA axis releases neuroendocrine hormones that increase available glucose and down-regulate non-essential processes. Chronic activation of the HPA axis occurs during repeated or chronic stress (Vedhara \& Irwin, 2007). Repeated or chronic stress response has been associated with an increase in circulating blood glucose, decreased insulin sensitivity, chronic inflammation, and abnormal fat metabolism, changes that often occur with prediabetes (Grundy, 2012; Wellen \& Hotamisligil, 2005).

\section{Work stress}

Work stress is a component of stress. Work stress can be measured as job strain (Karasek \& Theorell, 1998) or job imbalance (Seigrist, 2006). Empirical evidence indicates that work stress negatively affects physiologic and psychological health outcomes (Karasek \& Theorell, 1998; Marmot et al., 1978; Schnall et al., 2000; Siegrist, 2012). Years of sociological research have shown that workers experiencing work stress are at high risk for chronic illness and early mortality. Studies suggest that workers experiencing work stress have an increased risk for metabolic disease due to the physiologic impact of work stress (Biggi et al., 2008; Chandola, Brunner \& Marmot, 
2006; Fan, Blumenthal, Hinderliter \& Sherwood, 2012; Lansbergis et al., 2008; Seigrist Peter, Cremer \& Siedel, 1998) or to lack of participation in healthy behaviors Karlsson et al., 2003; Suwazono et al., 2009; Taris et al., 2011). Although less is known, there is some evidence that demonstrates work stress is directly associated with prediabetes when combined with other factors (e.g., shift work) (Biggi et al., 2008; Suwazono et al., 2009). However, the relationship between work stress and prediabetes has yet to be fully explicated.

\section{Health Behaviors}

The concepts that include prediabetes risk factors associated with healthpromoting behaviors were derived from synthesizing findings from empirical studies. The US Department of Agriculture (2010) recommends a daily diet that limits intake of sodium, fats, added sugars, and refined grains and emphasizes vegetables, fruits, whole grains, fat-free or low-fat dairy products, lean meats, beans and peas, and nuts and seeds in order to maintain a healthy diet. Evidence suggests that dietary excess of fatty acids derived from a dietary excess of sugared soft drinks, refined grains, and processed meats, (Buiso et al., 2007), a diet high in calories (Villegas, Salim, Flynn \& Perry, 2004), high in fat (Steyn et al., 2004), high in red meats (Demosthenes, 2005), or lower in fruits and vegetables (Villegas et al., 2004) may contribute to impaired glucose metabolism and insulin resistance. In addition to following a nutritional diet, the US Health and Human Services (USHHS) recommends regular, moderate-intensity exercise for at least 30 minutes per day on five days each week (US Health and Human Services [USHHS], 2008). Regular exercise that meets the USHHS guidelines prevents insulin resistance by increasing insulin sensitivity in target tissues (LaForge, 2013). During exercise, available triglyceride and low-density lipid resources are used, which, in turn, lower the total 
triglycerides and low-density lipid levels of the individual (LaForge, 2013). The ADA lists poor sleep (sleeping less than 6.5 hours/night or greater than 7.4 hours/night) as a potential risk factor for prediabetes (ADA, 2013). Sleep deprivation (six hours or less) has been shown to affect glucose regulation, appetite regulation (Buxton, 2010; Knutson et al., 2006; Knutson et al., 2007), glucose metabolism, and insulin sensitivity (Byberg et al., 2012; Gangwisch et al., 2007; Punjabi et al., 2004).

\section{Metabolic Profile}

The metabolic profile components include physiologic processes that are often present with prediabetes. Prediabetes screening and diagnostics statements from ADA, ACE, and ACCE suggest screening for high blood pressure (> 120/70), overweight (BMI $>25)$, low high-density lipids $(<40 \mathrm{mmol} / \mathrm{L})$, high triglycerides $(>150 \mathrm{~m} / \mathrm{dL})$, and high cholesterol (> 200mg/dL) if prediabetes is confirmed (ADA, 2013; Bloomgarden, 2008a; Garber et al., 2008). Empirical evidence has not shown a directional or dependent relationship among the components of the metabolic profile, but multiple studies have shown that these metabolic changes often occur simultaneously (Abdul-Ghani \& DeFronzo, 2009; Rao et al., 2004). A history of gestational diabetes and polycystic ovarian syndrome has also been associated with metabolic disease. It was beyond the scope of this dissertation study to measure all the prediabetes risk factors, especially those with lower incidence. The prevalence of gestational diabetes in the US is $2-10 \%$ (USHHS, 2011). The prevalence of polycystic ovarian syndrome in US women of reproductive age is 5\% (Solomon, 1999). Therefore, gestational diabetes and polycystic ovarian syndrome were not included in this study. The components of the metabolic profile included in this study were also limited by the biologic information contained in the participants' electronic medical records that was reviewed during data collection. 


\section{Prediabetes}

For several decades, a period of abnormal glucose metabolism that does not yet meet the requirements for T2DM has been recognized. This interim period is currently known as prediabetes. Prediabetes is a state of abnormal glucose metabolism in which either blood glucose levels are high, insulin sensitivity is low, or both. In 2003, the ADA established diagnostic criteria for prediabetes. There are three diagnostic criteria for prediabetes, fasting blood glucose, oral glucose tolerance, or glycated hemoglobin. One measure for prediabetes was assessed in this study, fasting blood glucose. Variables included in this study were limited by the data available in the participant's electronic record. An oral glucose tolerance test takes multiple hours to complete (ADA, 2013) and is not necessary for confirming a diagnosis. Only one of the criteria is necessary for diagnosing prediabetes (ADA, 2013). Therefore, fasting blood glucose which was available in the participant's electronic medical records, was included in this study.

In Figure 1, the model demonstrates relationships between and among the concepts and their relationship to prediabetes. There are several variables within each concept. Conceptual definitions of each concept, the variables within the concepts, and the proposed relationships are described next.

\section{Concepts ANd Proposed Relationships}

Prediabetes is a multidimensional condition. Concepts within the model include socio-cultural factors, stress, work stress, health behaviors, and coping. The outcome variable, prediabetes, is situated within a larger metabolic profile. Each concept includes variables that are hypothesized to contribute to prediabetes risk. The Conceptual Model for Metabolic Risk proposes several relationships. The direct relationship between work stress and prediabetes and the potential mediating factors (health behaviors, coping) are theorized. Each proposed relationship is discussed below. 


\section{Socio-Cultural Factors}

The socio-cultural concept includes: age, sex, race/ethnicity, family history, education, job role, and job hours. Based on The Conceptual Model for Metabolic Risk, socio-cultural factors are related to three concepts: work stress, health behaviors, and prediabetes within the metabolic profile.

\section{Socio-Cultural Factors and Work Stress}

Some socio-cultural factors have a known association with work stress. Lower status job role (Marmot et al., 1978) and nighttime job hours (Suwazono et al., 2009; Szolslang, 2010) are associated with increased work stress. Lower status workers tend to have lower education. Some studies use level of education as a proxy for socio-economic status (SES). Work stress has been shown to have a relationship with SES (Lahelma et al., 2010; Landsbergis et al., 2013). Other socio-cultural factors may have an interactive effect with health behaviors and work stress. Older workers experiencing stress are more likely to be sedentary, (Fransson et al., 2012), overeat (Nishitani, Sakakibara \& Akiyama, 2009), smoke, or use more alcohol (Biggi et al., 2008; Kuovonen et al. 2007). The role of sex, race/ethnicity, and family history on work stress has not been reported.

\section{Socio-Cultural Factors and Health Behaviors}

There is some evidence of an association between socio-cultural factors and health behaviors. Older age and male gender may reduce participation in exercise (Levi, Segal, St. Laurent \& Kohn, 2011). Ethnic minorities may have dietary and exercise practices that may differ from recommended guidelines (Brownson \& Boehmer, 2004; Levi et al., 2011; Shutlz et al., 2005; Swensen et al., 2005). As stated previously, lower status job roles and job hours are associated with detrimental health behaviors. Lower education is associated with poorer overall health and higher likelihood of chronic illness 
(Fonseca \& Zheng, 2011). The association between family history and health behaviors has not been reported.

\section{Socio-Cultural Factors and Prediabetes within the Metabolic Profile}

All of the socio-cultural factors have a relationship with prediabetes. As age increases, the risk of prediabetes increases (Chui et al., 2000). Risk for prediabetes appears to be equally divided between genders (ADA, 2013c). Non-White ethnicity, e.g., African-American, Hispanic, or Asian, increases the risk for T2DM (ADA, 2013); however, prevalence for prediabetes is equally divided among non-White ethnicity (CDC, 2011). Having a first-degree relative with a history of T2DM increases risk of prediabetes (Wagner et al., 2013). For every additional year of education beyond high school, risk for overall poor health is reduce by $2.7 \%$ and risk for T2DM is reduced by $0.8 \%$ (Fonseca \& Zheng, 2011). Lower status job role (Karasek et al., 1981; Karasek et al., 1987; Karasek \& Theorell, 1990; Marmot, et al., 1978) and nighttime or overtime job hours (Biggi et al., 2008; deBacquer et al., 2009; Karlsson et al., 2003; Suwazono, 2009) have been associated with metabolic changes consistent with prediabetes and other metabolic processes that occur with prediabetes.

\section{Stress}

The stress concept includes overall stress and work stress, a type of stress. Based on The Conceptual Model for Metabolic Risk, stress is related to coping. Work stress is related to two concepts: health behaviors and prediabetes within the metabolic profile. Work stress has a bi-directional relationship with health behaviors, as each affects the other. 


\section{Stress and Work Stress}

Stress is the result of any event in which external (environmental) or internal demands strain the adaptive potential of an individual. Stress can be acute or chronic. Stress activates release of neuroendocrine hormones, e.g., epinephrine, norepinephrine, or cortisol. Stress hormones can increase blood glucose, increase hepatic glucose production, increase lipolysis (fat cell destruction), or decrease insulin utilization (Surwit \& Schnieder, 1992; Surwit \& Williams, 1996), which may be necessary during acute stress. Chronic stress leads to persistent release of neuroendocrine hormones and leads to altered glucose metabolism, inflammation, and insulin sensitivity (prediabetes) (Lloyd, Smith \& Weinger, 2005). Overall stress has been shown to have a relationship with glucose metabolism (Williams et al., 2013) and with components of the metabolic profile, including prediabetes (Eriksson et al., 2010).

Work stress is a type of stress affecting most US workers (up to 80\%). More than $40 \%$ of US workers report chronic stress at work (Leka \& Jain, 2010). Work stress results in activation of the stress hormones linked to prediabetes (Vedhara \& Irwin, 2007). Work stress can also affect the way a worker thinks, feels, and behaves (Cox et al., 2000).

\section{Stress and Coping}

Coping is the physical and psychological response to a stressor in an effort to master the demands of stress and return to homeostasis (Hutchinson, 2011). Coping may be positive and reduce stress (problem-solving) or may be negative and increase the effects of stress (avoidance). In an effort to cope with stress, individuals may rely on social support (Hutchinson, 2011). Based on The Conceptual Model for Metabolic Risk, coping is related to prediabetes as part of the metabolic profile. 


\section{Work Stress and Prediabetes within the Metabolic Profile}

Work stress has a relationship with prediabetes, which is situated within the metabolic profile. Repeated or chronic work stress increases the risk for prediabetes by activating stress hormones (Karasek \& Theorell, 1990). An individual experiencing work stress is more likely to be overweight (Biggi et al., 2008; Brunner, Chandola \& Marmot, 2006; Karlsson et al., 2003) or have high blood pressure due to chronic stress (Karlsson et al., 2003; Vrijkotte, 2000). Dyslipidemia, a consequence of the stress response through altered fat metabolism, is independently associated with work stress (Siegrist, Cremer \& Seidel, 1997). Limited evidence has shown work stress to be associated with untoward changes in glucose metabolism within the metabolic profile (deBacquer et al., 2009; Suwazono et al., 2009) and with prediabetes (Li et al., 2013; Xu et al., 2012). Work stress may also have an interactive effect with health behaviors and prediabetes.

\section{Work Stress and Health Behaviors}

An individual experiencing work stress is likely to participate in health risk behaviors (Kuovonen et al., 2007). Individuals reporting high work stress, high job demands and low job control, have been shown to have a reduced, perceived control over exercise behavior (Lin et al., 2012; Payne, Jones \& Harris, 2005) and are less likely to participate in exercise in general (Fransson et al., 2012; Kuovonen et al., 2005). Both can lead to an increase in sedentary behavior. Work stress and diet are also related. Individuals with high work stress are more likely to overeat (Nishitani et al., 2009) or eat "high density foods" such as sweets (Payne et al., 2007, p. 127). Overeating or eating unhealthy foods can lead to overweight. Individuals experiencing work stress have also reported sleep disturbance (Akerstedt, Nordin, Alfredsson, Westerholm \& Kecklund, 2012; DeLange et al., 2009) and a higher probability for insomnia (Nakata et al., 2004). Workers experiencing work stress are more likely to smoke or overuse alcohol (Biggi et 
al., 2008; Kuovonen et al. 2007). Unhealthy diet, lack of exercise, lack of sleep, smoking and overuse of alcohol have been associated with an increased risk for prediabetes.

\section{Health Behaviors}

The health behaviors concept includes the following variables: diet, exercise, sleep, smoking, and alcohol. Based on The Conceptual Model for Metabolic Risk, health behavior is related to two concepts: work stress and prediabetes within the metabolic profile. Work stress has a bi-directional relationship with health behaviors. Prediabetes and health behaviors also have a bi-directional relationship. Each affects the other.

\section{Health Behaviors and Work Stress}

Health behaviors have an interactive relationship with work stress. The relationship between health behaviors and work stress has been previously described.

\section{Health Behaviors and Prediabetes within the Metabolic Profile}

A diet that is rich in fruits, vegetables, lean proteins, complex carbohydrates, and meets caloric needs maintains health (USDA, 2010). An unhealthy diet that is high in fat or calories alters metabolism and increases the risk for prediabetes (Buiso et al., 2007; Demosthenes, 2005; Steyn et al., 2004; Villegas et al., 2004). Thirty minutes of moderate to vigorous exercise most of the days of the week (five of seven) is recommended to maintain health (USHHS, 2008). Lack of participation in exercise alters the metabolism and increases the risk for prediabetes (LaForge, 2013). Sleep is essential for health by providing homeostatic recovery. Eight hours of sleep are recommended nightly (CDC, 2013). Lack of sleep alters glucose metabolism and increases the risk of prediabetes (Buxton, 2010; Knutson et al., 2006; Knutson et al., 2007). Smoking and overuse of alcohol have been associated with overall poor health, including prediabetes, by weakening the immune system (ADA, 2013; AHA, 2013; CDC, 2014). 
A diet rich in fat and calories, sedentary lifestyle, and chronic poor sleep increase the risk of overweight or obesity (large body size) (USHHS, 2012). High BMI and large waist size increase the risk for prediabetes (Antuna-Puente et al., 2008; Kahn \& Flier, 2000). High fat or high calorie diet, lack of exercise, poor sleep, smoking, and overuse of alcohol increase the risk for high blood pressure and dyslipidemia (Steyn et al., 2004; USHHS, 2012). High blood pressure is associated with prediabetes (Abdul-Ghani \& DeFronzo, 2009, Buiso et al., 2007; Petersen et al., 2006; Rao et al., 2009). High triglycerides and low high-density lipids are also associated with prediabetes (ADA, 2013; Bloomgarden, 2008a; Garber et al., 2008).

\section{Coping}

For the purposes of this study, coping refers to the psychological approach to manage stress. Based on The Conceptual Model for Metabolic Risk, coping is proposed as a factor related to prediabetes within the metabolic profile.

\section{Coping and Prediabetes within the Metabolic Profile}

The relationship between coping and prediabetes is unknown. However, limited evidence suggests that negative coping strategies increase the risk for prediabetes by reducing glucose tolerance (Fujunishi et al., 1998). Negative coping strategies have been associated with working harder and working despite illness (Astvik \& Mellen, 2012). Working without adequate rest may increase the overall risk of illness, including prediabetes.

\section{Prediabetes within the Metabolic Profile}

Based on The Conceptual Model for Metabolic Risk, prediabetes is within a larger metabolic profile. Prediabetes is the interim period between normal glucose metabolism and severely altered glucose metabolism (T2DM). During this period of 
altered glucose metabolism (prediabetes) an individual has degrading pancreatic beta cells and a decreased capacity to utilize glucose effectively (Abdul-Ghani \& DeFronzo, 2009). Along with metabolic changes associated with glucose metabolism, evidence has demonstrated that other metabolic processes occur simultaneously. Persons with prediabetes are more likely to have large body size (overweight or overfat), have high blood pressure, and dyslipidemia (ADA, 2013a; Bloomgarden, 2008a; Garber et al., 2008). Large body size, high blood pressure and dyslipidemia are risk factors for cardiovascular disease (e.g., myocardial infarction, stroke) (AHA, 2013). Based on The Conceptual Model for Metabolic Risk, prediabetes is related to health behaviors (described previously). If left untreated prediabetes leads to T2DM (ADA, 2013a). Consequences of untreated components of the metabolic profile (T2DM and cardiovascular disease) are beyond the scope of this study.

\section{Conceptual And Operational Definitions}

\section{Socio-Cultural Factors}

Socio-cultural factors include genotypic and environmental variables. Genotypic variables included: age, sex, and family history. Environmental variables included: education, job role, and job hours. Race/ethnicity is both a genotypic and environmental variable.

Age

Age is the numerical representation of time since birth. Age was measured by noting date of birth entered by the participant on the online, demographic survey. 
Sex

Gender is the categorization of either male or female based on genotype or phenotype assigned at birth. Gender was measured by noting sex the participant's self report of male or female sex on the online, demographic survey.

\section{Race/Ethnicity}

Ethnicity is the categorization of persons based on phenotype. The major categories are: White, Black, Asian/Pacific Islander, American Indian/Alaskan Native and Hispanic (The White House, 1995). Ethnicity was measured by noting selfidentification of ethnicity category indicated by the participant on the online, demographic survey.

\section{Family History}

For the focus of this study, family history was defined as significant, family medical history focused on genetic and personal characteristics that have a direct link to risk for prediabetes and T2DM, e.g., first-degree relatives with T2DM. Family history was measured by noting the number of first-degree relatives with T2DM as reported by the participant on the online, demographic survey.

\section{Education}

For the focus of this study, education is the highest level of completed education in an institute of learning. Education was measured by noting the highest level of education entered by the participant on the online, demographic survey.

\section{Job Role}

For the focus of this study, job role refers to the title or designation of job duties assigned to the participant by the computer company. Job role was measured by noting the job title or job duties entered by the participant on the online, demographic survey. 


\section{Job Hours}

For the focus of this study, job hours refers to the typical hours of work assigned to the participant by the computer company. Job hours was measured by noting start and stop times entered by the participant on the online, demographic survey.

\section{Stress}

Stress is a physiological, psychological, or social response an actual or potential threat. Stress can be acute or chronic (Hutchinson, 2011). For the purposes of this study, stress refers to the global and event-specific stress. Stress was measured online through the Perceived Stress Scale (PSS).

\section{Work Stress}

Work stress is a type of stress that occurs when there is a mismatch occurs between a worker's skills and abilities and the demands or pressures of the job or when the worker is unable to cope with the demands or pressures of the job (Leka \& Jain, 2010). For the purposes of this study, work stress was conceptualized in two ways: as job strain and as job imbalance. Job strain is the interaction effect of work demands and decisional latitude (Karasek \& Theorell, 1990; Karasek et al., 1981). It is the effect of physical or psychological demands at work coupled with inadequate decisional control over work duties (Schnall et al., 2009). Job strain was measured online by the Job Content Questionnaire (JCQ) survey. Job imbalance is based on social relationships at work and the worker's perceived effort, reward, and their interaction effects (Siegrist, 1995). When a worker perceives high effort and low gains in return (Siegrist, 1996), the worker experiences an imbalance, or failed social reciprocity. This disparity causes job imbalance (Seigrist, 2012). The Effort-Reward-Imbalance Scale Questionnaire (ERIQ) 
measured job imbalance online. Descriptions of the two components of job strain are given next.

\section{Job Demands}

Work demands are the measurement of how hard a worker works. Demands include psychological and physical components. They can include performance expectations and pressures on performance. Work demands are also the degree of role ambiguity and the amount of organizational constraints placed on task completion (Karasek et al., 1998). Work demands was measured as part of the JCQ on the online survey.

\section{Job Decisional Latitude}

Decisional latitude is the operationalization of the authority to make decisions at work combined with the opportunity to use and develop skills on the job (Schnall et al., 2009). It is the combined effect of decision authority and skill discretion. In other words, decisional latitude is a measure of the worker's ability to control assigned work activities through work decisions and individual control (Karasek \& Theorell, 1990). Decisional latitude was measured as part of the JCQ on the online survey.

\section{Effort}

Based on an expectation of social reciprocity, a worker puts forth effort. A worker's effort has intrinsic and extrinsic components. Effort includes the physical and psychological energy expended by the worker in order to meet job demands. Job demands include job role, work hours (including overtime), job responsibilities, and work relationships. Effort can be high or low (Siegrist, 2014). Effort was measured as part of the ERIQ on the online survey. 


\section{Reward}

The worker has an expectation that their effort will be matched by an expected occupational reward in order to maintain balanced social reciprocity. Rewards can result from personal interactions or employee benefits. Rewards include social status, fair treatment, control over job duties, promotions, job security, and salary. Rewards can be high or low. Reward was measured as part of the ERI on the online survey.

\section{Health Behaviors}

Health behaviors include conscious actions known to affect health. The behavioral variables that were included in this study are: diet, exercise, sleep, smoking, and alcohol. Variables included those that are critical for health, optional for health, and detrimental to health. Eating (diet) and sleeping are physiologically necessary for sustaining health. Exercise and moderate alcohol intake have health benefits, and both abstinence and excess are detrimental to health. Smoking at any level of intake is detrimental to health.

\section{Diet}

For the purposes of this study, diet refers to selection or limitation of the amount and type of food a person eats in order to meet the dietary quality recommendations from the US Department of Agriculture (USDA, 2010). Diet was assessed through the online Rapid Eating and Activity Assessment for Patients (REAP-S) survey.

\section{Exercise}

For the purposes of this study, exercise refers to current participation in a program of exercise for 30 minutes per day most of the days of the week as recommended by the US Department of Health and Human Services (USHHS, 2008). Exercise was measured with the online Godin Leisure Time Exercise Questionnaire (GLTEQ). 


\section{Sleep}

Sleep is a natural period of suspension of consciousness where rest and inactivity occur and the body restores energy levels. Sleep was measured online with the Insomnia Severity Index (ISI) survey.

\section{Smoking}

For the purposes of this study, smoking refers to the use of tobacco in cigarettes. Any use of cigarette smoking is detrimental to health (USHHS, 2014). Smoking was measured as part of the online, demographic survey.

\section{Alcohol}

Use of alcohol is defined as consuming beverages that contain alcohol (beer, wine, spirits). Moderate use of alcohol (up to 6/week) has health benefits. Abstinence from alcohol and heavy consumption ( $>6$ drinks/week) are detrimental to health (NIAA, 2014). Alcohol was measured as part of the online, demographic survey.

\section{Coping}

Coping is an effort to manage the impact of stress through feelings, thoughts, and actions (Hutchinson, 2011). For the purposes of this study, coping is the psychological approach to stress management. Coping was measured through the online The Brief Approach/Avoidance Coping Questionnaire (BACQ).

\section{Metabolic Profile}

For the purposes of this study, metabolic profile includes the outcome variable, prediabetes (described below) and other metabolic changes that commonly occur with prediabetes. The HC nurse collected metabolic profile data from a blood sample sent for laboratory analysis as part of the yearly, biometric exam. Exam data was entered into the 
participant's electronic medical record. Data was extracted from the participant's electronic medical record (EMR).

\section{Prediabetes}

Prediabetes is the interim period between degrading glucose metabolism and diagnosis of T2DM. Its hallmark signs are impaired fasting glucose, impaired glucose tolerance, or both (Abdul-Ghani \& DeFronzo, 2009; Rao et al., 2004). The NI HC nurse measured prediabetes by obtaining a fasting blood glucose sample that was sent for laboratory analysis. If the fasting blood glucose result was abnormal, a second fasting blood glucose sample was to be collected, however, employees chose not to return for the second fasting blood glucose test. The results were extracted from the EMR.

\section{Body Size}

For the purposes of this study, two measures of body size, body mass index (BMI) and waist circumference, determined body size. Body mass index was used to determine weight status and status of health. The BMI score was calculated by using the standard formula calculation using the height and weight measures taken during the biometric exam by the HC nurse. The circumference of a person's waist was used to determine their central adiposity. The HC nurse measured height, weight, and waist circumference. The results were extracted from the electronic medical record EMR.

\section{Dyslipidemia}

Two lipids have been identified as most significant for prediabetes risk, highdensity lipids and triglycerides (ADA, 2013). High-density lipids are a densely packed lipoprotein. The primary function of high-density lipids is to remove low-density lipids and triglycerides by transporting them to the liver for removal. They also maintain integrity and function of the lining of blood vessels. High-density lipids are primarily 
found in plant-based fats. Serum laboratory tests revealing low HDL indicate a diet with poor vegetable lipoprotein intake (American Association for Clinical Chemistry, 2013). High-density lipids were measured from a blood sample sent for laboratory analysis by the HC nurse. The results were extracted from the EMR.

Triglycerides are made when excess food intake from fats and carbohydrates are converted into fatty acids (AHA, 2013). The excess intake of fatty acids leads to storage of fat in adipose tissue in the form of triglycerides. If excess fat and carbohydrate intake continues, storage of triglycerides is also found in non-adipose tissues such as muscle, liver, and the pancreas (Buiso et al., 2007). Triglycerides were measured from a blood sample sent for laboratory analysis by the HC nurse. The results were extracted from the EMR.

\section{Blood Pressure}

Blood pressure is the force of blood against the arteries. Systolic blood pressure measures the pressure in the arteries when the heart muscle contracts; diastolic blood pressure measures the pressure in the arteries between heartbeats (during rest and refilling). Blood pressure is normal when systolic blood pressure is less than $120 \mathrm{mmHg}$ and diastolic blood pressure is less than $80 \mathrm{mmHg}$ (American Heart Association [AHA], 2012). The HC nurse measured blood pressure and the results were extracted from the EMR.

\section{ASSUMPTIONS}

The assumptions for this study include:

- $\quad$ Prediabetes has multiple contributing factors.

- $\quad$ Contributing factors to prediabetes are related.

- Job strain is a harmful type of work stress. 
- The electronic medical record is accurate.

- $\quad$ Biometric screening procedures are accurate.

- $\quad$ Laboratory values are accurate.

- $\quad$ Participants are truthful in their responses to the questions asked by the HC nurse during the biometric screening appointment.

- $\quad$ Participants are truthful in their responses on the online survey administered for this study.

\section{LIMITATIONS}

The conclusions of this study will not be generalizable to all US workers in industrial settings because the convenience sample will be obtained from only one central Texas employer. The study will be cross-sectional in design, and participants will be limited to work experiences at a single setting. No cause and effect conclusions can be drawn.

\section{SUMMARY}

This descriptive study aimed to assess the prevalence of prediabetes and work stress in an adult population working in a technical industry. The conceptual model that guides this study was derived from a thoughtful combination of expert recommendations and empirical evidence. The data gathered from this study helped to further explicate the direct relationship between work stress and prediabetes in an adult, technical industry worker population. In addition, the relationships among socio-cultural factors, stress, health behavior, coping, and prediabetes were explored. Analysis provided useful information about the prevalence of prediabetes and the associated risk factors, including work stress. Identification of prediabetes in a worker population will raise individual and worksite awareness and provide the opportunity to design educational and counseling 
interventions aimed at modifying amenable risk factors in order to prevent or delay the progression to Type 2 diabetes mellitus. 


\section{Chapter Two}

\section{GENERAL INTRODUCTION}

In order to appreciate the state of the science regarding prediabetes and its associated risk factors, a review of the literature was completed. The review of literature led to the development of a conceptual model for this study, the Conceptual Model for Metabolic Risk. The Conceptual Model for Metabolic Risk demonstrates proposed relationships between and among the concepts identified as risk factors through the review of literature and the relationship of each concept to prediabetes, the outcome variable (Figure 1). The structure and organization in the following literature review sections are based on the Conceptual Model for Metabolic Risk.

Figure 1. Conceptual Model for Metabolic Risk

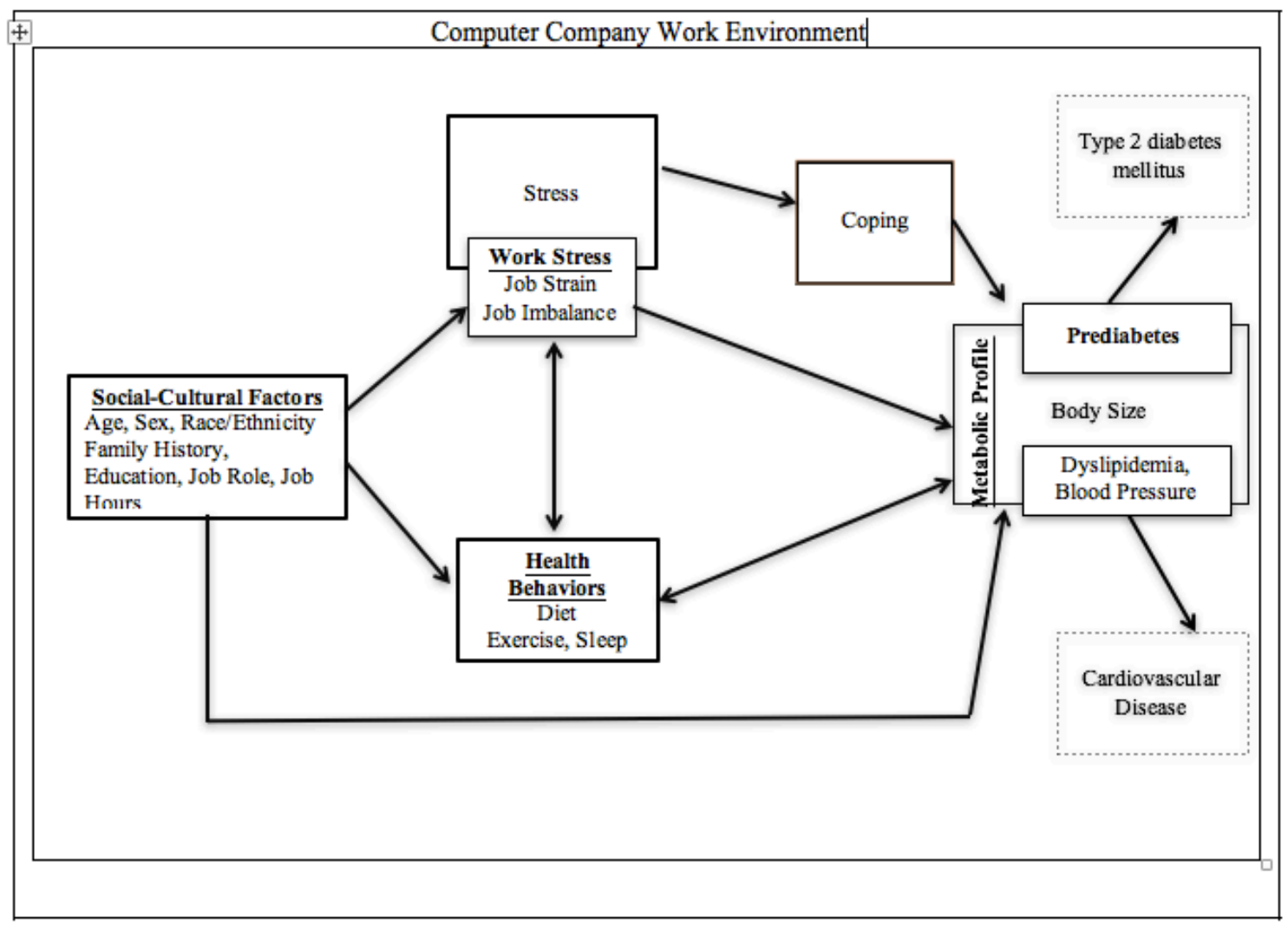


The Conceptual Model for Metabolic Risk reflects a basic ecological approach that situates the individual within a larger environment. The focus of this study was on the development of a metabolic profile in individuals who work within a technical, industrial setting. The review of literature revealed that prediabetes is a complex, metabolic disease with multiple risk factors. Several metabolic changes often occur simultaneously with prediabetes. As such, prediabetes is shown within a cluster of metabolic health outcomes, labeled metabolic profile. The metabolic profile contains several health outcomes that are known to coincide with prediabetes in most cases and may even contribute to severity of illness. These coinciding health outcomes are precursors to $\mathrm{T} 2 \mathrm{DM}$ and cardiovascular disease and include: large body size, dyslipidemia, and high blood pressure.

Based on the review of the literature, the concepts in the Conceptual Model for Metabolic Risk that are directly related to risk for prediabetes are: socio-cultural factors, stress, coping, and health behaviors. The relationships between socio-cultural factors and prediabetes, stress and prediabetes, and health behaviors and prediabetes are well established and supported by empirical data (ADA, 2013; Bloomgarden, 2008a; Buxton, 2010; Garber et al., 2008; Knutson et al., 2006; Knutson et al., 2007; Surwit et al., 1996). Empirical studies show that there is a bi-directional relationship between health behaviors and work stress (Crispim et al., 2011; Fransson et al., 2012; Knudsen, Ducharme \& Roman, 2007; Kouvonen et al., 2007; Payne, Jones \& Harris, 2005). Some socio-cultural factors (e.g., age, education) have a direct relationship with work stress (Marmot et al., 1978). Work stress, a common type of stress, affecting $40 \%$ of US workers (Sauter et al., 2002), is hypothesized to have a direct relationship to prediabetes. Empirical studies also suggest that work stress may have a direct relationship to physiological, metabolic processes that often accompany prediabetes, including high-than-recommended waist 
circumference, high body mass index, dyslipidemia, high blood pressure, and impaired glucose metabolism (Biggi et al., 2008; Brunner et al., 2007; Chandola et al., 2006; deBacquer et al., 2009; Fujino et al., 2006; Karlsson et al., 2003; Suwazono et al., 2009). The variables within the Conceptual Model for Metabolic Risk may have interactive effects. Socio-cultural factors (age, sex, race/ethnicity, family history, education, job role, job hours) combined with work stress (job strain and job imbalance) and along with untoward health behaviors or habits, such as poor diet, lack of exercise, poor sleep quality, smoking, and alcohol use, as well as non-productive coping mechanisms lead to the development of a metabolic profile that is the precursor to T2DM and cardiovascular disease. The literature that follows presents the concepts within the model. It begins with a description of the central Texas computer technology company, the work environment in which the individual risks for developing a specified metabolic profile (prediabetes, larger-than-recommended body size, dyslipidemia, and elevated blood pressure) were manifest.

\section{COMPUTER COMPANY}

Historically, studies about work stress and work situations and their potential relationships with metabolic conditions have been set in large industrial or technical settings (Marmot et al., 1978). The central Texas computer technology company provided a comparable setting with which to explore relationships between risk factors and prediabetes for this study. The selected company is within the Professional, Scientific, and Technical Services sector. The Bureau of Labor and Statistics (2014) Professional, Scientific, and Technical Services sector designation is for companies that specialize in performing professional, scientific, and technical services for other companies, including development and production of computer systems and design. Founded in the mid-1970s, 
the computer technology company manufactures test and measurement hardware and software systems. The computer company employs more than 7,000 persons worldwide and is headquartered in central Texas. It has full-time, part-time, and temporary positions. Positions within the company include office/clerical, graphic design, engineering, information technology, manufacturing, marketing, web design, sales and services. The computer company has been named one of Fortune's Best Places to Work for the last 15 years. The voluntary turnover rate is only $5 \%$. The most common salaried position is software engineer. The most common hourly position is technician. The company employs mostly White males. Only $25 \%$ of employees are minorities, and $24 \%$ are women. Salaried employees enjoy a flexible work schedule, including working from home. Operating hours are weekdays with no overnight shifts.

\section{Prediabetes within a Metabolic Profile}

Prediabetes is the interim period between normal glucose metabolism and T2DM (Abdul-Ghani \& DeFronzo, 2009). Normal glucose metabolism requires prompt increase of available insulin by pancreatic beta cell activation plus adequate insulin sensitivity in the liver and muscles in order to metabolize available blood glucose (Banerjee \& Cruikshank, 2004). During prediabetes there are no definitive signs and symptoms, yet physiologic changes in glucose metabolism have begun. Two, hallmark physiologic changes occur: (1) impaired fasting glucose and (2) impaired glucose tolerance. One or both may be present. Impaired fasting glucose occurs when blood glucose levels remain elevated after a period of fasting for at least eight hours. Impaired fasting glucose has two distinct characteristics: fasting plasma glucose levels are higher than normal (> $100 \mathrm{mg} / \mathrm{dL}$ ) and there is an exaggerated early increase in plasma glucose immediately after a meal (at thirty minutes). On the other hand, impaired glucose tolerance is a result 
of the body's inability to utilize glucose after ingesting food or sugared drink. Impaired glucose tolerance presents with two signs: (1) there is a rapid increase in plasma glucose following meals, with continued increase in plasma glucose concentration (after 60 minutes) and (2) there is a lack of decline in plasma glucose two hours after ingestion of a meal or sugared drink. The prevalence of impaired fasting glucose, impaired glucose tolerance, or the combination of both in prediabetes is known to vary depending on age and ethnicity (Abdul-Ghani \& DeFronzo, 2009; ADA, 2013; Nathan et al., 2007). The incidence of T2DM is more likely when both prediabetes processes, impaired glucose tolerance and impaired fasting glucose, occur together (Banerjee \& Cruickshank, 2004; Santaguida et al., 2005).

Though subtle, all of the physiologic processes during glucose metabolism occurring in prediabetes can be measured. Although other metabolic factors may be present and can be measured (i.e., high blood pressure, dyslipidemia), these are not required for confirmation of prediabetes. Prediabetes is confirmed through one of three laboratory tests (ADA, 2013). The Expert Committee on Diagnosis and Classification of Diabetes Mellitus adjusted references ranges in 1997, 2003, and 2010 (ADA, 2010). The most current laboratory test ranges for a prediabetes diagnosis are as follows: (1) fasting blood glucose test results ranging from 100-126mg/dL; (2) oral glucose tolerance test results ranging from 140-200mg/dL; and (3) glycated hemoglobin results ranging from 5.7-6.5\% (ADA, 2013a). For the purposes of this study, fasting blood glucose and glycated hemoglobin will be measured as indicators of this primary outcome, prediabetes.

Along with changes in glucose metabolism, evidence suggests that other metabolic changes are concurrent with prediabetes. Decades of research by the American Diabetes Association (ADA) and other leading agencies, such as the American College of Endocrinology/American College of Clinical Endocrinology (ACE/ACCE) and the 
American College of Endocrinology (ACE), have acknowledged that prediabetes is often present among other factors within a metabolic profile (ADA, 2013a; Bloomgarden, 2008a; Garber et al., 2008). Each of these factors is discussed next.

\section{Body Size: Waist Circumference ANd Body Mass IndeX}

The relationship between body size and prediabetes is well documented (Geis et al., 2010; Haffner et al., 2000; Rhee et al, 2006). Two measures of body size were included in this study, body mass index (BMI) and waist circumference. Body mass index is a calculation of body size and body fatness using a person's height and weight $\left(\mathrm{BMI}=\right.$ pounds $/$ inches $\left.^{2} \mathrm{X} 703\right)$. The result is used to determine body size category $(<$ 18.5 underweight, 18.5-24.9 normal weight, 25.0-29.9 overweight, > 30 obese) (CDC, 2014). When a healthy weight is exceeded (overweight or obesity), the fat accumulation promotes a chronic low-grade inflammation. The overabundant adipocytes (fat cells) become hormonally active. Adipocytes release inflammatory hormones that mimic the effects of stress, such as inflammation of blood vessels, hyperlipidemia, hyperglycemia, and insulin resistance. Adipocytes can also regulate metabolic homeostasis and the immune response (Kahn \& Flier, 2000; Wellen \& Hotamisligil, 2005). Obesity is the most common cause of insulin resistance (Petersen et al. 2006), one of the underlying processes of prediabetes.

By examining population level data from the 2006 NHIS, Rolka, Burrows and Geiss (2008) reported the prevalence of prediabetes increased with weight status. Using standard BMI ranges, prediabetes was found in $2.3 \%$ of normal weight individuals (< $\left.25 \mathrm{~kg} / \mathrm{m}^{2}\right)$, in $3.9 \%$ of overweight individuals $\left(25-30 \mathrm{~kg} / \mathrm{m}^{2}\right)$, and in $6.3 \%$ of obese individuals $\left(\geq 30 \mathrm{~kg} / \mathrm{m}^{2}\right.$ ) in a representative sample of the US population (Rolka, et al., 
2008). The study by Rolka et al. (2008) was based on population-level data that uses robust methodology and has generalizable results to the US population.

In addition to general overweight, the distribution of fat in the abdominal region (central adiposity) has been linked to altered glucose metabolism (Antuna-Puente et al., 2008; Neeland et al., 2012; Rosmond et al., 1998). There is a dose-response between fattiness and insulin resistance, but the relationship is greater with fat concentrated in the abdominal region, possibly because these adipocytes are more hormonally active (Kahn \& Flier, 2000). Central adiposity increases the risk for developing T2DM by increasing insulin resistance. Insulin resistance raises the amount of available insulin. The resulting increase in circulating insulin increases demand for glucose and promotes adipocyte activation, which causes an unregulated increase in triglyceride stores and fatty acid uptake in order to meet demands. This results in even greater insulin resistance (AntunaPuente et al., 2008; Kahn \& Flier, 2000; Rosmond et al., 1998).

In a study by Hackworth et al. (2009) the anthropometric, biochemical, psychosocial, and behavioral profiles of people with prediabetes were compared. This lifestyle intervention study was conducted across three regions in Australia and included Chinese and Anglo-Australian participants. Participants included a bi-ethnic sample of 261 Australian men and women. Baseline anthropometric measures included BMI and waist-to-hip ratio. The results suggested that participants with prediabetes were not only obese (BMI $30 \mathrm{~kg} / \mathrm{m}^{2}$ ), but the waist-to-hip ratio was higher than recommended (measuring $>80 \mathrm{~cm}$ for males and $>90 \mathrm{~cm}$ for females) (Hackworth et al., 2009). Although data collection was rigorous, including anthropometric measures, blood tests and self-report, the results may differ in other ethnic groups within Australia or in another country owing to differences in diet, daily activities, or other daily routines. 
In the US, the San Antonio Heart Study by Haffner et al. (2000) determined that body weight and fat distribution were associated with conversion to T2DM, even after controlling for age and gender. Anthropometric measures were used to predict the development of T2DM in Mexican Americans who were normoglycemic at baseline. Participants included 721 Mexican-American men and women aged 25-64 living in San Antonio, Texas. The data showed that body weight, BMI, and waist circumference all positively predicted T2DM, however, waist circumference was the strongest predictor. Participants who developed T2DM had significantly higher waist-to-hip ratios, when compared to those who did not progress to T2DM. The risk for T2DM in those with the highest waist-to-hip ratio was 11 times greater than those in the lowest quartile (95\% CI, 4.2 - 28.8). The study results by Haffner et al. (2000) are limited to Mexican-American residents of San Antonio, Texas.

Similarly, Neeland et al. (2012) investigated the association between fat accumulation and risk for developing T2DM in Dallas, Texas. The Dallas Heart Study included a population-based cohort of 732 multi-ethnic (White, African-American, Hispanic), obese adults $\left(\mathrm{BMI} \geq 30 \mathrm{~kg} / \mathrm{m}^{2}\right)$ without $\mathrm{T} 2 \mathrm{DM}$. Body size was calculated using BMI and muscle-fat distribution using magnetic resonance imaging (MRI). Risk for T2DM was measured by fasting blood glucose and glycated hemoglobin. Higher fat mass in the central regions $(\mathrm{OR}=1.48,95 \% \mathrm{CI}, 1.17-1.88)$, insulin level $(\mathrm{OR}=1.34,95 \% \mathrm{CI}$, 1.06-1.70), and weight gain over the 7-year follow-up (OR 1.06, 95\% CI, 1.02-1.10) were independently associated with prediabetes among participants with normal glucose at baseline. Over the 7-year follow-up, the median increase in weight for those who developed prediabetes was $4.5 \mathrm{~kg}$ and $7.2 \mathrm{~kg}$ for those progressed to $\mathrm{T} 2 \mathrm{DM}$, while the median increase in weight for those who did not develop prediabetes or T2DM was $1.6 \mathrm{~kg}$ 
$(p<0.001)$. The researchers concluded that central adiposity increases the risk for prediabetes and T2DM.

Based on multiple, empirical studies across various geographic settings, the association between weight and blood glucose outlined in the pathogenesis reports is well recognized. Overweight, especially if weight is concentrated in the central region of the body, is a significant risk factor for prediabetes. The pathogenesis reports by Kahn and Flier (2000), Goldberg (2009), and Antuna-Puente et al. (2008) outline the basic pathway related to the pathophysiological and etiological relationships between: overweight and high glucose and fat distribution and high glucose across populations. The selected empirical studies support these pathogenesis reports. All of the studies used populationbased data, which indicate vigorous measures and generalizable conclusions. The population-level data reported by Rolka et al. (2008) is based on the 2006 US NHIS study. The population-based study by Hackworth et al. (2009) included a large, bi-ethnic Australian sample $(n=261)$. The results reported by Haffner $(2000)$ were derived from the population-based study San Antonio Heart Study based in San Antonio, Texas. The results by Neeland et al. (2012) are based on a population cohort study using a large, multi-ethnic sample in Dallas, Texas ( $\mathrm{N}=732$ participants) over a seven-year period. It is reasonable to conclude that measures of body size are concurrent, metabolic factors that are related to prediabetes; all of the studies presented illustrate how general weight status and weight distribution are associated with prediabetes. Therefore, it was reasonable to include body size as part of the metabolic profile that includes measures of prediabetes.

\section{DYSLIPIDEMIA: HDL AND TRIGLYCERIDES}

Although it is unclear how dyslipidemia directly affects prediabetes, there is evidence that a relationship exists. Two measures of dyslipidemia were included in this 
study: high-density lipids and triglycerides. High-density lipids (HDL) are a densely packed lipoprotein. The ratio of protein to lipids is three to one, making this lipoprotein an efficient and effective delivery system for essential fatty acids. The primary function of HDL is to remove low-density lipids by transporting them to the liver for removal and to maintain integrity and function of the endothelium of blood vessels. High-density lipids are primarily found in plant-based fats. Serum laboratory tests revealing low HDL indicate a diet with poor vegetable lipoprotein intake (Abdul-Ghani \& DeFronzo, 2009; Buiso et al, 2007, AHA, 2013c). Triglycerides are fatty molecules that are derived from vegetable oils and animal fats (Clarke, 2011). From a physiologic perspective, prediabetes often begins with an excessive dietary intake of fatty acids in the form of high-sugared or high-fat foods. This excessive intake of fatty acids promotes deposition of triglycerides in adipose (fat) tissue. Persistent fatty acid intake and excess fat in adipose tissue leads to fat deposition in non-adipose tissues such as muscle, liver, and the pancreas. This increase in fat deposition may have a dose-dependent relationship with insulin sensitivity, even in healthy subjects (Bardini et al., 2010; Buiso et al., 2007). Screening for dyslipidemia is recommended when prediabetes is confirmed (ADA, 2013a). Empirical evidence suggests a relationship between dyslipidemia and prediabetes as part of a larger metabolic profile.

Zhang et al. (2008) investigated the relationship between dyslipidemia and glucose in a large, 15-cohort European study conducted in eight countries. The population-based study included 8,960 male and 10,516 female participants and showed that the prevalence of low HDL and high triglycerides in those with impaired fasting glucose or impaired glucose tolerance comprised $26-33 \%$ compared to a rate of $24 \%$ in participants with normal glucose. When compared to participants with normal glucose, men and women with impaired fasting glucose had significantly higher triglycerides $(p<$ 
0.01). After adjusting for age and cohort, overall dyslipidemia worsened with rising fasting glucose $(p<0.01)$. The results showed a significant positive relationship between fasting blood glucose and triglycerides $(\beta=0.14$ and 0.12 for men and women, respectively), and a significant negative relationship between fasting blood glucose and $\operatorname{HDL}(\beta=0.06$ and 0.03 for men and women, respectively).

Bardini et al. (2010) aimed to assess the association between 1-hour plasma glucose and markers of inflammation (white blood cells and dyslipidemia) in a cohort of 1,062 male and female Italian participants. None of the participants had a history of cardiovascular disease, diabetes, nor other inflammatory diseases. The results indicated that participants with either prediabetes or a high, normal glucose level had a worsening lipid profile. The triglyceride to HDL ratio was significantly increased in persons with either prediabetes or a high, normal glucose result when compared with those who had a low, normal glucose result $(p<0.05$ overall). Insulin sensitivity was also significantly lower in participants with prediabetes and high, normal glucose $(p<0.01)$. The researchers reported that participants with a high 1-hour plasma glucose result (> $155 \mathrm{mg} / \mathrm{dL}$ ) had a five-fold risk of T2DM when compared to participants with 1-hour plasma glucose below $155 \mathrm{mg} / \mathrm{dL}$.

The Zhang et al. (2008) and Bardini et al. (2010) studies were both conducted in Europe. The DECODE study by Zhang et al. (2008) was conducted in Finland, and the cohort study by Bardini et al. (2010) was conducted in Italy. The Zhang et al. study was population-based and included 15 European countries. These results could be generalized to a European population. The Bardini et al. study included a large number of participants in a single European country. Their results were similar to the larger population-based study. Participant ethnicity was not reported in either study. It is possible that the results may differ within Europe in individuals with different ethnic or cultural backgrounds. 
Similarly, results would vary outside of Europe due to differences in ethnicity and culture. However, one US study showed similar results.

In a large study using data from the population-based San Antonio Heart Study, Haffner, Mykkanen, Festa, Burke and Stern (1999) investigated how dyslipidemia affects the progression to T2DM. Participants included 1,734 bi-ethnic (White and Hispanic) men and women aged 25-64 living in San Antonio, Texas. The results showed that persons with prediabetes had lower HDL and higher triglycerides than their normoglycemic counterparts, even after adjusting for age and body mass $(p<0.001)$. Participants with prediabetes were also more likely to convert to T2DM if they had lower than recommended HDL and higher than recommended triglycerides $(p<0.001)$. The 7year follow-up data $(N=195)$ showed that persons with prediabetes who converted to T2DM had significantly higher triglycerides and significantly lower HDL than nonconverters $(p<0.001)$. The results are limited to a single city in the southwestern United States, and, therefore, cannot be generalized to the US or other countries. However, these results are consistent with the European results.

Based on the empirical data, the relationship between dyslipidemia and prediabetes remains consistent across geographical locations. The prevalence study by Zhang et al. (2008) showed that dyslipidemia is associated with prediabetes using a large, population-based sample across Europe (19,476 participants). The study by Bardini et al. (2010) was set in Italy with 1062 participants, yet data also demonstrated a strong relationship between dyslipidemia and prediabetes. Similarly, Haffner et al. (1999) outlined a significant relationship between dyslipidemia and prediabetes using a bi-ethnic (White and Hispanic) sample of 1,734 participants in San Antonio, Texas. Although it is unclear how dyslipidemia, including low HDL and high triglycerides, is linked to 
prediabetes, evidence suggests that a relationship is present. Therefore, it was reasonable to measure dyslipidemia as part of a metabolic risk profile that includes prediabetes.

\section{BLOOd Pressure}

Blood pressure (BP) is the amount of pressure exerted by the blood against the arteries measured when the heart beats (systolic) and when the heart rests (diastolic) using a sphygmomanometer. The result is used to determine normal blood pressure, prehypertension (higher-than-normal blood pressure), and hypertension (abnormally high blood pressure) (National Institutes of Health, 2012). For the purposes of this study, high blood pressure referred to any result that is higher than normal (systolic $>120 \mathrm{~mm} / \mathrm{Hg}$ or diastolic $>80 \mathrm{~mm} / \mathrm{Hg}$ ). The relationship between $\mathrm{BP}$ and blood glucose is well known. High blood pressure and high blood glucose are related to the metabolic response to chronic stress (physical or psychological) (Grundy, 2012). The association between BP and prediabetes is based on decades of research data consistently report high BP and high blood sugar occur simultaneously (Abdul-Ghani \& DeFronzo, 2009; ADA, 2013a; Buiso et al., 2007; Petersen et al., 2006; Rao et al., 2009). Screening for high blood pressure is recommended when prediabetes is confirmed (ADA, 2013a). It was important to include BP as part of the metabolic profile that includes measures of prediabetes.

The US Agency for Healthcare Research and Quality (AHRQ) sponsored a systematic review to assess the negative health outcomes of individuals with prediabetes. The empirical evidence included research from 1978 to 2004. The meta-analysis data reported by Santaguida et al. (2005) for the AHRQ showed that there is an increased relative risk for cardiovascular disease, which includes high BP and cardiovascularassociated mortality in persons with prediabetes. In persons with impaired fasting glucose 
(prediabetes), the pooled relative risk for cardiovascular mortality, a consequence of sustained high BP, ranges from 1.19-1.66 (95\% CI, 1.36-1.96) (Santaguida et al., 2005).

Using population data from the San Antonio Heart Study, Haffner et al. (1999) showed that in a sample of 1,734 normoglycemic Mexican-American and White men and women who later converted to T2DM had significantly higher BP. Measures used to determine glucose metabolism included fasting glucose and fasting insulin. Blood pressure readings were taken three times during each visit over the 7-year study. The average BP was used. Normoglycemic participants who converted to T2DM at follow-up had higher systolic blood pressure $(117.6 \pm 0.4$ vs. $123 \pm 1.0, p<0.001)$, higher diastolic blood pressure $(71.6 \pm 0.2$ vs. $74.7 \pm 0.7, p=0.005)$. The results remained significant after adjusting for age, sex, ethnicity, fasting glucose, and body size ( $p=0.016$ for systolic and $p=0.042$ for diastolic blood pressure). The longitudinal, population-based study used rigorous measures that may be generalizable. However, the study was set in a single city in the southwestern US with a bi-ethnic sample. Results may differ in other regions of the US, with people from different ethnicities or cultures.

Gupta et al. (2008) compared the blood pressure variability of normal, healthy individuals with individuals with prediabetes. The participants included 12 AfricanAmerican and Caucasian men and women with a family history but no personal history of T2DM. Blood pressure was measured over seven days using an ambulatory, electronic device. Glucose status (normoglyemic vs. prediabetes) was determined by oral glucose tolerance test. The participants were divided equally between the normoglycemic (healthy) and prediabetes groups. The data showed that asymptomatic, overweight adults with prediabetes $(n=6)$ had abnormal BP variability when compared with their overweight, healthy counterparts without prediabetes $(n=6)$. Four out of the six participants with prediabetes had abnormal BP variability and consistently higher BP 
readings, while none of the normoglycemic participants $(n=6)$ had abnormal BP variability $(p<0.001)$. The data showed that, overall, $66.7 \%$ of participants with prediabetes had abnormal BP variation while none of the participants with normal glucose had abnormal BP $(p<0.001)$. Results of this study are significant. Glucose status was determined the gold standard oral glucose tolerance test. Blood pressure was measured over the course of seven days.

Despite the differences in sample size and ethnicity, both Haffner et al. (2000) and Gupta et al. (2008) concluded that untoward blood pressure changes occur with prediabetes using rigorous anthropometric measures and laboratory analyses. The sample size included by Haffner et al. (2000) was much larger than the sample size for Gupta et al. (2008), 1734 vs. 12, respectively. The longitudinal, population based study by Haffner et al. (2000) included a large sample size, but the study was limited to San Antonio, TX. The cross-sectional study by Gupta et al. (2008) had a small sample size and limited to Baton Rouge, Louisiana. A larger sample size may yield different results. Both studies were limited to the southern US; it is possible that the results of both studies may differ in another region within the US or outside of the US. Both studies included White participants and one other ethnicity. Gupta et al. (2008) included African-Americans, whereas Haffner et al. (2000) included Mexican-Americans. Results may differ with other ethnic groups.

Although it is unclear whether blood pressure is a risk factor for prediabetes, evidence suggests that high blood pressure and high blood glucose often occur simultaneously (Abdul-Ghani \& DeFronzo, 2009, Buiso et al., 2007; Petersen et al., 2006; Rao et al., 2009). Concurrent high blood pressure may also contribute to overall risk of mortality (Santaguida et al., 2005). Therefore, it was reasonable to measure BP as part of a metabolic risk profile that includes prediabetes. 


\section{Treatment AND GoAlS}

The treatment and goals for prediabetes are focused towards management of metabolic risk factors through lifestyle modifications (DPP, 2012). The overall goal is to reduce blood glucose and improve insulin sensitivity (Bloomgarden, 2008). Lifestyle modification is the cornerstone of treatment (Garber et al., 2008). A modest reduction in body weight (i.e., $7 \%$ loss) has been shown to improve glucose metabolism and, in some cases, return glucose to normal (DPP, 2012). Moderate participation in exercise can improve insulin sensitivity and lower blood glucose (Colberg et al., 2010; DPP, 2012). Reducing body weight and increasing exercise can also lower BP and improve dyslipidemia (Bloomgarden, 2008). Lifestyle modification can be achieved through education, counseling, and personal discipline. However, some risk factors for prediabetes, such as age or family history, are non-modifiable.

\section{SUMMARY}

The changes in glucose metabolism that occur in prediabetes can be associated with several metabolic risk factors. Metabolic risk factors such as larger body size, high $\mathrm{BP}$, dyslipidemia are related to the stress response and the inflammatory changes that occur with chronic stress (Wellen \& Hotamisligil, 2005). These factors are modifiable through participation in health behaviors such as healthy diet, regular exercise, and adequate sleep. Chronic stress and the response to stress can also be modified. Work stress, a type of acute and chronic stress, has long been associated with negative changes in health (Biggi et al., 2008; deBacquer et al., 2009; Karsek, 1979; Karlsson et al, 2003; Marmot et al., 1978) and can also be modified. Other risk factors are not modifiable and based on individual social or cultural factors. Based on the review of literature, the following types of risk factors were found: socio-cultural factors, health behaviors, and work stress. Socio-cultural concepts and related variables are described next. 


\section{Socio-Cultural Factors}

Age

The ADA recommends screening for prediabetes in all overweight individuals aged 45 years or older (ADAa, 2013). The probability for T2DM increases as age increases. It is estimated that approximately $37 \%$ of US adults have prediabetes (CDC, 2014). Based on 2014 data for diagnosed and undiagnosed diabetes, prevalence across age groups are as follows: approximately $4 \%$ of adults aged $20-44$ years, nearly $16 \%$ in the 45-64 age group, and nearly $26 \%$ in the 65 years and older group, the highest of any age group (CDC, 2014). Prevalence data for prediabetes alone is not available, however, it is possible that the prevalence distribution for prediabetes is similar.

In a study by Chui, Lee, Cohan, and Chuang (2000) the researchers examined the effect of age on insulin sensitivity. The data was derived from the larger, populationbased Genetics of Non-Insulin Dependent Diabetes Mellitus Study (GENNID) study in the US. The sample included 149 normotensive, glucose tolerant (normal glucose) men and women aged 19-78. Insulin sensitivity was measured using oral glucose tolerance test, fasting blood sugar, and homeostasis model assessments tests. The homeostasis model was used to calculate insulin sensitivity and pancreatic beta cell function. Stepwise regression failed to show a relationship between age and insulin sensitivity. The data showed a significant effect of age on pancreatic beta cell function. After controlling for body mass index, blood pressure, insulin sensitivity, the results indicated an independent association between age and pancreaticbeta cell function in adults aged 19 - 78, regardless of insulin sensitivity. The results demonstrated that pancreatic beta cell function declines at a rate of $1 \%$ per year $(p<0.001)$. Although the data was derived from a larger, population-based dataset, this study included a small participant sample. Results may differ using a larger sample. 
Prevalence estimates for distribution of prediabetes and diabetes among age groups reported by the CDC are derived from large, population-based studies such as The National Health and Nutrition Examination Survey (NHANES) and the National Health Interview Survey (NHIS). Population studies use robust data collection techniques that are generalizable. Both national surveys confirmed diagnosis of diabetes and prediabetes using fasting blood glucose and glycated hemoglobin (CDC, 2014). The study by Chui et al. (2000) used a smaller sample $(n=149)$ derived from a large, population-based study (GENNID) using a multi-ethnic sample (White, Hispanic, Black, Japanese) in the US. Based on prevalence distributions and population-based data, age is a significant nonmodifiable risk factor for prediabetes. The likelihood of having prediabetes increases as age increases. Therefore, it was important to include age as a socio-cultural risk factor for prediabetes.

$\operatorname{Sex}$

The prevalence of prediabetes is equally distributed between males and females. Even after progression to T2DM, the prevalence remains approximately equal between genders, with US males at $11.8 \%$ and females at $10.8 \%$ (ADA, 2013c). However, the underlying, physiologic processes occurring during prediabetes may differ between genders. Women with prediabetes are more likely to have impaired glucose tolerance while men are more likely to have impaired fasting glucose (Abdul-Ghani \& DeFronzo, 2009; Nathan et al., 2007). There may also be gender differences in the prevalence of associated risk factors common in prediabetes. Men and women may have differing proinflammatory profiles.

In the pathogenesis report by Adbul-Ghani and DeFronzo (2009), the authors describe the pathophysiology and etiology of prediabetes. Abdul-Ghani and DeFronzo 
(2009) report pathophysiological gender differences in glucose metabolism based on four population-based studies. The population data show that, in individuals younger that age 55, women are more likely to have impaired glucose tolerance, or higher blood glucose after meals, whereas men are more likely to have impaired fasting glucose, or higher blood glucose after a period of fasting for at least 8 hours. The report also states that prevalence of impaired glucose tolerance and impaired fasting glucose increases with age but gender distribution was not reported.

Saltevo, Kautiainen, and Vanhala (2009) used a cross-sectional, population-based approach to examine gender differences in inflammatory markers in East Finland. The study included 923 middle-aged men and women (mean age $46 \pm 6$ ) with no exclusion criteria. Glucose tolerance was measured using fasting blood glucose and oral glucose tolerance test. Results were used to categorize participants into normal glucose tolerance, prediabetes, or T2DM. Measuring lipid profiles, adiponectin, high sensitivity C-reactive protein (hs-CRP), and interleukin-1 receptor antagonist (IL-IRA), indicated inflammation. Prevalence of prediabetes among men was $28 \%$ and T2DM was diagnosed in $9 \%$. Among women, prevalence of prediabetes was $15 \%$ and $4 \%$ were diagnosed with T2DM. The differences in prevalence were statistically significant ( $p<0.001$ for both). The data showed that women have significantly higher pro-inflammatory markers than men. In participants with normal glucose tolerance, adiponectin concentrations were higher in women than men. The ratio between women and men was 1.61 (95\% CI, 1.48$1.75, p<0.001)$. The mean ratio between women to men with prediabetes was $1.57(95 \%$ CI, 1.36-1.85, $p=0.011)$ and 1.16 for T2DM (95\% CI, 0.87-1.53, $p=0.011)$. Women with prediabetes and T2DM had significantly higher concentrations of hs-CRP ( $p=$ $0.026)$ and IL-IRA $(p=0.011)$ compared with men with prediabetes. The results showed that mean gender ratios increased linearly from normal glucose tolerance to prediabetes 
for hs-CRP ( $p=0.019$ and $p=0.013$, respectively), indicating significantly higher proinflammatory markers among women with abnormal glucose metabolism. The researchers reported the cross-sectional design and the extended storage of samples $(\sim 5$ years) as a limitation of the study. The study was also limited to an eastern Finland population.

The cross-sectional study by Saltevo et al. (2009) was conducted in East Finland. The pathogenesis report is based on multiple empirical studies that provide insight into pathophysiological and epidemiological aspects of prediabetes. Both illustrate the metabolic gender differences that occur during prediabetes. Therefore, despite the nearly equal distribution of prediabetes prevalence between genders, it was important to include gender as a socio-cultural risk factor for prediabetes due to the potential differences in physiologic expression.

\section{Race/Ethnicity}

Including race/ethnicity as a risk factor prediabetes was based on significant differences in T2DM prevalence in ethnic minorities when compared to non-Hispanic Whites (ADA, 2013). The ADA, ACE/ACCE Task Force and the ACE Prediabetes Conference Consensus list ethnicity as a potential risk factor for prediabetes. These expert agencies agree that ethnic minorities should be screened for prediabetes due to higher overall incidence and prevalence of prediabetes and subsequent progression to T2DM (ADA, 2013a; Bloomgarden, 2008b; Garber et al., 2008). In 2012, an estimated 86 million (37\%) American adults aged 20 years and older had prediabetes (CDC, 2014). After adjusting for age and gender differences, distribution of prediabetes among ethnicities is as follows: $35 \%$ prevalence in non-Hispanic Whites, 39\% prevalence in non-Hispanic Blacks, and 38\% prevalence in Hispanics (CDC, 2014). After progression 
to T2DM, the distribution shifts. In 2012 , the prevalence of T2DM was: $7.6 \%$ of nonHispanic Whites, $13.2 \%$ of non-Hispanic Blacks, and $12.8 \%$ of Hispanics (CDC, 2014). The absolute prevalence of prediabetes is hypothesized to be ethnic dependent (AbdulGhani \& DeFronzo, 2009). Empirical studies have mixed results regarding the association of ethnicity and prediabetes.

Using a longitudinal study design, Dagogo-Jack et al. (2014) assessed the rate and predictors of prediabetes and whether racial disparities were a significant factor during the transition to prediabetes over a 5.5-year period. The bi-ethnic (White and Black) sample included 343 men and women aged 18-65 living in Tennessee, US. The participants were mostly female (70\%) with a mean age of $44.2 \pm 10.6$ years. At enrollment, the participants were normoglycemic and did not have a current T2DM diagnosis, but each participant had one or both parents who were diagnosed with T2DM. Most participants $(86 \%)$ had one parent with T2DM. The proportion of participants who reported one or both parents with T2DM did not vary by race. During quarterly visits: anthropometric measures, lipid profile, and glucose measures (fasting blood glucose, oral glucose tests, glycated hemoglobin, fasting blood insulin) were collected. The cumulative incidence of both prediabetes and T2DM was similar between races $(35 \%$ and $30 \%$ among White and Black participants, respectively) $(p=0.2997)$. Survival probability did not differ by race $(p=0.7855)$. The researchers reported that incident prediabetes was 1012.5 cases per 100 for both White and Black participants. There were no significant effects of race on incident prediabetes, even after adjusting for baseline characteristics. The longitudinal design was the strength of the study. Behaviors and habits can change over time, yet no differences were noted after 5.5 years. The limitations of this study include a single US city and including only Whites and Blacks. The conclusions are also limited to individuals with a family history of T2DM. Results may differ in another 49 
region within and outside of the US, with a different race/ethnic population, and in persons without family history of T2DM.

Results opposing the findings by Dagogo-Jack et al. were reported by Kodama et al. (2013). Using a systematic review and meta-analysis approach, the researchers assessed ethnic differences in insulin sensitivity and insulin response. The sample was consisted of 174 journal articles. Studies that included the insulin sensitivity and acute insulin response to glucose indices and limited to one race/ethnicity were included. The results indicated an ethnic variation in the distribution of insulin sensitivity and acute insulin response across all subgroups. Blacks were found to have significantly lower insulin sensitivity and higher insulin response compared to Whites and East Asians ( $p=$ 0.028). East Asians were found to have higher insulin sensitivity $(p<0.005)$ compared to Whites and Blacks and a lower insulin response than Whites $(p=0.017)$. Meta-analysis is a robust research methodology. Limitations included limiting articles to select variables. In addition, the researchers reported a variation in data quality across journal articles. Results are further limited by narrowing journal article selection to studies that included only one race/ethnicity.

The prevalence distribution among race/ethnicities reported by the CDC is based on large population-based studies. Despite the nearly equal distribution of prediabetes prevalence among ethnicities, there are slight differences. The disparity is greater when T2DM is diagnosed. Therefore, it was important to consider race/ethnicity as a sociocultural risk factor for prediabetes, primarily because of the differences in prevalence of T2DM (i.e., higher prevalence in non-Hispanic Black and Hispanic populations than in the White population). It is possible that there are underlying, physiological differences in prediabetes expression or progression to illness (T2DM) related to ethnicity. 


\section{Family History}

As with age and race/ethnicity, there is strong evidence that family history significantly contributes to risk for prediabetes. The ADA (2013) states that genetic (family) history increases the overall risk for T2DM. Ninety to ninety-five percent of T2DM is associated with family history of T2DM (CDC, 2004). Therefore, the ADA recommends screening for prediabetes if individuals have one or more first-degree relatives with a history of T2DM (ADA, 2013). In a review by Petersen and Shulman (2006), the researchers report the lifetime risk for developing T2DM in lean, young offspring of parents with T2DM was 40\%. Empirical studies support this conclusion.

Jensen et al. (2002) used data from the ADA's GENNID Study to determine the prevalence of normal glucose, impaired fasting glucose, impaired glucose tolerance, and diabetes in participants with first-degree relatives diagnosed with T2DM. A total of 531 healthy, middle-aged men and women (White, Black, Hispanic, Asian) without a prediabetes or diabetes diagnosis with at least one first-degree relative diagnosed with T2DM were included. Measures included BMI, BP, waist-to-hip ratio, fasting blood glucose, oral glucose tolerance, and fasting blood insulin. The data showed that approximately $27 \%$ of participants had previously unknown impaired glucose tolerance. Two percent had impaired fasting glucose. Eight percent had both impaired glucose tolerance and impaired fasting glucose. Nineteen percent had previously undiagnosed diabetes $(p<0.001$ for all). The findings show an undiagnosed diabetes prevalence of $19 \%$ in this sample compared to $7.3 \%$ reported by the US NHANES III. The data was extracted from a population-based data set and can be generalized to a US population. However, the sample was relatively small. Results may vary with a larger sample or outside of the US. 
Similarly, Wagner et al. (2013) concluded that family history of diabetes was strongly associated with prediabetes. The sample included 8,106 healthy men and women who reported at least one first-degree relative with T2DM. Data was collected at four centers across Germany. Family history was determined through self-report. Measures of glucose metabolism included fasting blood glucose, oral glucose tolerance test, and fasting blood insulin. Data was analyzed for each study center and as a meta-analysis. Family history of T2DM was associated with a $40 \%$ increased risk for prediabetes for each single study and the meta-analysis (OR 1.40, 95\% CI, 1.27-1.54, $p<0.001)$. After adjusting for age, BMI, and gender, the association remained significant at $26 \%$ (OR $1.26,95 \% \mathrm{CI}, 1.14-1.40, p \leq 0.001)$. The population-based data can be generalized to a German population. Race/ethnicity was not reported. Results may vary outside of Germany or within populations of differing race/ethnicity.

The evidence supporting race/ethnicity as a risk factor for prediabetes is based on large, population-based studies supported by the ADA and other agencies. The conclusion by Peterson and Shulman (2006) was based on a review of four studies that reviewed etiology and pathophysiology markers. Jensen et al. (2002) derived data from the larger GENNID study using a multi-ethnic sample. Although the meta-analysis by Wagner et al. (2013) reported data from mostly females in Germany, the data showed a strong association between family history and prediabetes in each of the four studies and the meta-analysis and the association remained significant even after adjusting for sex, age, and BMI as confounders. Consequently, it was important to consider family history of T2DM as a significant, socio-cultural risk factor for prediabetes. 


\section{Education}

Level of education has shown to have a causal relationship with health (Cutler \& Muney, 2007; Fonseca \& Zheng, 2011) and mortality (Cutler \& Muney, 2007). Level of education has also been shown to affect health through participation in health behaviors (Brunello, Fort, Sheenweis \& Winter-Ebner, 2011).

In a population study by Fonseca and Muney (2011), the researchers aimed to determine causal relationships between education and health. Data was drawn from three population surveys across 13 countries. Datasets included the US Health and Retirement Study (HRA), the English Longitudinal Study on Ageing (ELSA), and The Study of Health and Ageing and Retirement in Europe (SHARE) study. Levels of education were adjusted across countries to provide comparison data. The results indicated a marginal effect of years of education on health. Higher levels of education were associated with lower levels of reported health. For every year after high school education, levels of reported level of poor health decreased by $2.8 \%$, reported impairment in levels of activities of daily living decreased by $1 \%$, and reported T2DM decreased by $0.8 \%$ ( $p<$ 0.05), even after controlling for age, gender, and country. In addition, each year of additional education beyond high school resulting in decreased likelihood of dying in the subsequent 10 years by $3.6 \%(p<0.05)$. Population studies have robust methodology. The results can be generalized to the population. Datasets from thirteen countries were included in this study.

In another population study by Brunell et al. (2011), a multi-country European data set to was used determine effect of education on health and the role of health behaviors. Population data from 12 European countries (SHARE and ELSA) were included. Measures focused on education, health, and health behaviors in a sample of men women aged 50 and older. Health behaviors included smoking, drinking alcohol, 
exercising, and healthy diet. Analyses showed that each additional year of education beyond high school reduced self-reported poor health by $7.1 \%$ for females and $3.1 \%$ for males. The effects of education on health behaviors accounted for $23-45 \%$ of the effect of education on health, suggesting an intermediary effect. Population studies are robust, and results can be generalized to the population. Results from this study are limited to a European population.

Level of education may affect job type, with higher education reducing the likelihood of hazardous, low-wage, or reduced autonomy at work (Feinstein et al., 2006). Lower status workers have increased work stress, which increases risk for poor health (Marmot et al., 1978). In a cross-sectional study by Landsbergis et al. (2003) the researchers examined the effects of socio-economic status on the association between job strain and blood pressure. The study included participants from the Work Site Blood Pressure Study in New York, a prospective study of psychosocial factors and ambulatory BP. Participants included 283 men, aged 30 - 60, from eight worksites in New York. Blood pressure was measured through an ambulatory BP monitor for 24 hours during a normal workday. Job strain measures included Karasek's Job Content Questionnaire and Jenkins Activity Survey. Other measures included demographic data, anthropometrics, use of alcohol, smoking, education, job title, and income. The men had an average of 14.3 years of education (range 6 - 18) and personal income of $\$ 50,629$ (range $\$ 17,500-\geq$ 100.000). Positive correlations were found between socio-economic status (SES) and both job demands $(r=28-0.36, p<0.05)$ and decisional latitude $(r=0.36-0.44, p<$ 0.05). Measures of SES were significantly associated with ambulatory BP. Ambulatory BP for blue-collar (low status) workers was 4.0mmHg (95\% CI, -2.4 - 10.3, $p<0.05)$ higher than BP for white -collar workers. Blue-collar workers with job strain had $11 \mathrm{mmHg}$ higher mean systolic BP $(95 \% \mathrm{CI}, 1.6-22.0, p<0.05)$ when compared to the 
systolic BP of white-collar men without job strain and $8.4 \mathrm{mmHg}$ higher mean systolic BP (95\% CI ,1.6 - 22.0, $p<0.05)$ when compared to men with job strain. Overall, the researchers concluded that among men exposed to job strain, a dose-response relationship was found between measures of SES (including education) and ambulatory BP. The results are limited to a male sample in New York, US. Data is further limited by the cross-sectional design and limited sample size. Results may differ in a larger sample, a prospective design, or female participants.

\section{Job Characteristics: Role and Hours}

Between 1967 and 1969 the Whitehall Study found significant, negative correlations between employment grade and cardiovascular disease and employment grade and mortality (Marmot et al., 1978). Nearly twenty years later sociologist Robert Karasek found that low status workers, especially with low education, those working in repetitive motion occupations, and workers with reduced control over work schedule, experience a specific type of work stress that predisposes the worker to physical and psychological ill health (Karsek, 1978; Karasek et al., 1981; Karasek, Gardell \& Lindell, 1987; Karasek \& Theorell, 1990).

Workers who generally perform repetitive operations with their hands, physical energy and physical skill comprise $18 \%$ of the United States (US) workforce. These lowstatus workers commonly sit or stand through most of their day and are generally assigned to shift work, including nights and weekends (US Department of Labor [USDOL], 2012). Low status workers typically have a higher body mass index, are more likely to smoke have untoward cardiovascular changes (Karlsson et al., 2003; Suwazono et al., 2009) and reduced sleep quality (Suwazono et al., 2009; Silversten et al., 2006), especially if working during the night (Biggi et al., 2008). Studies indicate that low status 
workers have an increased risk for hypertension due to flattened blood pressure variability (Fan, Blumenthal, Hinderliter \& Sherwood, 2012; Lansbergis et al., 2008); poor sleep (Suwazono et al., 2009; Silversten et al., 2006); metabolic syndrome due to inflammatory processes such as elevated cortisol and central adiposity (Chandola, Brunner \& Marmot, 2006); dyslipidemia due to altered fat metabolism (Seigrist Peter, Cremer \& Siedel, 1998); and impaired glucose tolerance, one of the hallmark signs of prediabetes (Karlsson et al., 2003; Suwazono et al., 2009).

Work hours and type of shift have been established as contributing to worker health (Biggi et al., 2008; deBacquer et al., 2009; Karlsson et al, 2003; Leka et al., 2004; Suwazono et al., 2009; Szosland et al., 2010). Day shiftwork, night shiftwork, swing shifts, and overtime have been shown to affect health. Night shift workers have increased risk of early cardiovascular changes due to higher incidence of overweight, alcohol consumption, smoking, and overall stress (Biggi et al., 2008). Type and amount of work hours can also affect work-life balance (Albertsen et al., 2008), cortisol secretion (Hansen et al., 2008; Rosmond et al., 2000), and participation in health-promoting behaviors (Taris et al. 2011). Work and its relationship to prediabetes will be discussed later in this chapter.

\section{Summary of Socio-Cultural Factors}

The relationship between socio-cultural risk factors, age, gender, race/ethnicity, and family history, and prediabetes is well documented (ADA, 2013; Bloomgarden, 2008a; Garber et al., 2008). The relationship between job characteristics and prediabetes is related to work stress and lack of participation in health promoting behaviors. Despite the increased risk with socio-cultural risk factors, occurrence of prediabetes is not guaranteed. Prediabetes is a multi-factorial condition with non-modifiable, socio-cultural 
factors and modifiable, behavioral risk factors (ADA, 2013; Bloomgarden, 2008a; Garber et al., 2008). Health behaviors that increase the risk for prediabetes include: poor diet, lack of exercise, inadequate sleep, smoking, or use of alcohol. Stress has also been associated with changes in glucose metabolism and predisposition to prediabetes (Grundy, 2012; Surwit et al., 1996; Wellen \& Hotamisligil, 2005). The literature supporting the relationships between stress and prediabetes and health behaviors and prediabetes are discussed next.

\section{Stress and Prediabetes}

Stress has frequently been identified as a risk factor for the development of T2DM (Surwit et al., 1996), especially in persons with other risk factors (Lloyd et al., 2005). During times of stress, the hypothalamic-pituitary-adrenocortical (HPA) axis becomes activated (Vedhara \& Irwin, 2007). Activation of the HPA axis releases neuroendocrine hormones that prepare an individual to cope with a stressor by increasing available glucose for energy and down-regulating non-essential processes (Vedhara \& Irwin, 2007). Chronic activation of the HPA axis (during persistent stress) perpetuates the stress response and leads to an increase in circulating blood glucose, decreased insulin sensitivity, chronic inflammation, and abnormal fat metabolism. These responses contribute to the metabolic changes that often occur with prediabetes (Grundy, 2012; Wellen \& Hotamisligil, 2005).

The cluster of metabolic factors within the metabolic profile may be interrelated through the stress response. Stress neuroendocrine hormones are released when the HPA axis is activated (Vedhara \& Irwin, 2007). Hormones increase blood glucose and decrease insulin sensitivity, hallmarks of prediabetes. Over production of stress hormones promotes inflammation, which results in thickening of arterial walls. This predisposes an 
individual to high blood pressure. An overproduction of stress hormones can alter fat metabolism, which leads to dyslipidemia (Black, 2003; Wellen \& Hotamisligil, 2005).

Using population-based data from the Australian Diabetes, Obesity and Lifestyle Study, Williams et al. (2013) examined the effects of stress on abnormal glucose metabolism. Participants included 3,759 men and women aged 25 and older with normal glucose at baseline. Baseline data was collected during 1999-2000. Follow-up data collection was five years later (2004-2005). Data collection included anthropometric measurements, lipid profiles, blood pressure, fasting glucose, oral glucose tests, socioeconomic data (education and income), family history of diabetes, and health habit data (smoking, exercise, food intake). Stress was measured through the Perceived Stress Scale and Life Events Scale. The results showed that women who developed abnormal glucose levels at follow-up reported higher levels of perceived stress at baseline $(p=0.010)$. Women who developed abnormal glucose metabolism also had lower levels of physical activity $(p=0.021)$ and central adiposity $(p<0.001)$, which compound the risk for prediabetes. After adjusting for age and education, the relationship between perceived stress and incident abnormal glucose in women was dose-dependent ( $p=000.5$ and $p=$ 0.003 , respectively). Women who reported high levels of stress at baseline were more likely to develop abnormal glucose metabolism over five years (OR 1.42, 95\% CI, 0.90 $2.24 ; 1.68,95 \% \mathrm{CI}, 1.06-2.66$; and $1.89,95 \% \mathrm{CI}, 1.19-2.99, p=0.003$ for lowest, middle, and highest perceived stress respectively). Perceived stress in men was not associated with abnormal glucose metabolism. Strengths of the study include longitudinal design and large sample size. Results can be generalized to an Australian population. Ethnicity was not reported. Results may change with varying ethnic populations within Australia and in countries or regions outside of Australia due to individual differences or cultural variation. 
In a similar study, Eriksson et al. (2010) tested associations of stressful life events and incidence of individual components of metabolic illness (high blood glucose, low HDL, high triglycerides, high waist circumference, high blood pressure) and as a cluster of any three (metabolic syndrome) using a cross-sectional design. Participants included a random, population-based sample drawn from The Prevalence, Prediction, and Prevention of Diabetes - Botina Study in Western Finland. A total of 3,621 men and women aged 18-78 were included. Glucose metabolism was assessed by fasting glucose, fasting insulin, and oral glucose tolerance tests. Results were used to calculate insulin resistance. Waist circumference, HDL, triglycerides, blood pressure, family history of diabetes, smoking habits, and alcohol intake were also measured. Stressful life events were measured through a 15-item survey. The results indicated that the odds of having metabolic syndrome were significantly higher among participants who reported experiencing extremely stressful life events with ongoing financial strain (OR 1.60, 95\% CI, $1.07-2.39, p=0.023$ ) or work overload (OR 1.17, 95\% CI, $0.83-1.65, p=0.381)$. When analyzed as individual components, participants who reported stressful life events in finance, work, or health-related areas had significantly higher levels of insulin resistance $(p=0.010)$, waist circumference $(p=0.001)$, BMI $(p=0.001)$, triglycerides $(p$ $=0.027)$, and impaired glucose tolerance $(p=0.008)$. The associations were not compounded by age, sex, smoking, alcohol intake, or family history of diabetes. The data were based on a population-based dataset and can be generalized to the Western Finnish population. The researchers reported the cross-sectional design and inclusion of only White participants as a limitation. The results are limited to a population in Finland. Results may vary in other ethnicities throughout Finland or in regions outside of Finland. 


\section{Summary of Stress and Prediabetes}

Stress as a risk factor for prediabetes is related to the physiological response that occurs during an assault from stress hormones. Stress hormones can raise blood sugar, decrease insulin sensitivity, and stimulate appetite. Stress hormones can also increase the likelihood of obesity by altering fat metabolism and inflammation stimulating hormonal activity within adipocytes and increasing blood vessel pressure. Obesity and inflammation are often present during prediabetes. Stress can include psychological stress (e.g., financial concerns), physical stress (e.g., illness) or components of both (e.g., work stress). As such, it was important to consider stress as a risk factor for prediabetes.

\section{Work Stress}

In the late twentieth century, the organization of work changed to a rigid system where work tasks became specialized and discrete (Taylorist model). The focus of work shifted to the efficiency and productivity of the organization rather than individuals, especially in industrial-type work. Workers were expected to follow assigned tasks and strive to achieve organizational goals, many times at the expense of autonomy and decision-making (Enoch, 2006). As control shifted to management, work became isolated (Schnall, Dobson \& Rosskam, 2009). During this time, social epidemiology research began to emerge (Vaananen, Anttila, Turtiainen \& Varje, 2012) as a branch of epidemiology that uses a bio-psychosocial paradigm (Honjo, 2004). Social epidemiology studies the distribution and determinants of health and disease in populations by assessing social, individual, and biologic factors (Honjo, 2004). The relationship between work stress and disease became an important topic in occupational health. Work stress was acknowledged as a major hazard that affected an employee's health and lowered his or her productivity (Schnall et al., 2009). Population studies began to focus on the effects of work stress on disease, including: the British Whitehall II study, the European Belgium 
Job Stress Project (BELSTRESS) Study, the US Framingham Offspring Study, and US Nurses Study. The shared focus of these studies was the relationship between health and environment (Vaananen et al., 2012). In 2006, the American Psychological Association (APA) (2011) began the yearly, population-based Stress in America Survey. The purpose of the survey is to measure the attitudes and perceptions of stress among the US general public and identify sources of stress, behaviors used to manage stress, and the economic and health impact of stress. Across studies, work stress has been recognized as a potential health threat.

The World Health Organization (WHO) defines a healthy job as one where the pressures placed on an individual are in accordance with their abilities and resources, where workers have control over their work, and have the support needed to perform well (World Health Organization [WHO], 2013). Pressures at work are unavoidable. Some stress at work is beneficial, however, repeated exposure to stress at work results in adverse psychological and physical health (APA, 2011; APA, 2013; Leka et al., 2004). Work stress occurs when a worker experiences demands or pressures that are mismatched with his skills and abilities or when the worker's ability to cope is challenged (Leka \& Jain, 2010). Work stress can be acute or chronic. Poor organization, poor work design, poor management, unsatisfactory conditions, or lack of support from co-workers and supervisors are potential sources of work stress (WHO, 2013). The APA's Center for Organizational Excellence (2013) report states that one-third of US workers experience chronic stress at work. Half of US workers state that the workplace does not value worklife balance, and one in four report that work demands interfere with their ability to fulfill home and family responsibilities. Low salaries (47\%), lack of opportunity for growth and advancement (44\%), heavy workload (39\%), long hours (38\%), and undefined work expectations (36\%) are sources of work stress in the US worker (APA, 2013). 
Work stress triggers the stress response through acute and chronic activation of the sympathetic nervous system (Vedhara \& Irwin, 2007). Chronic activation of the sympathetic nervous system increases risk for illness or injury (EFILWC, 2007; Schnall, Dobson \& Rosskam, 2009; WHO, 2007). Psychological symptoms of work stress include: anger, irritability, less motivation to exercise, coping through smoking or use of alcohol, and changes in sexual drive; physical symptoms of work stress include: fatigue, muscle tension, poor digestion, increased appetite, increased pulse and blood pressure, increased blood sugar, and insulin resistance (APA, 2014). Sociological and epidemiological research has found that workers experience a specific type of stress (work stress), especially in workers with low education, those working in repetitive motion occupations, workers with reduced control over work schedule (Karasek et al., 1981; Karasek et al., 1987; Karasek \& Theorell, 1990; Marmot, et al., 1978), white-collar workers (Webster \& Bergman, 1999) and workers in the information technology industry (Dhar \& Dhar, 2010; Webster \& Bergman, 1999).

For several decades, empirical studies have shown the association between work stress and health outcomes (Marmot et al., 1978; Karasek \& Theorell, 1998; Schnall et al., 2000). Historically, research has focused on the effects of work stress on cardiovascular disease and early mortality. Cardiovascular disease is a complex, slowprogressing illness that begins with subtle, often unnoticeable signs. Type 2 diabetes mellitus is similar to cardiovascular disease in its subtlety, slow progression, and multidimensional presentation. Both have inflammatory components and are associated with the stress response. The socio-cultural and behavioral factors associated with each are similar. However, whereas there is extensive, empirical literature to support the link between work stress and cardiovascular disease, there are fewer studies that focus on the relationship between work stress and prediabetes. 
Between 1967 and 1969 the Whitehall Study found significant, negative correlations between job type and cardiovascular disease and between job type and early mortality (Marmot, et al., 1978). In the decades that followed, work stress was associated with markers of metabolic disease: altered glucose metabolism (deBacquer et al., 2009; Suwazono et al., 2009), insulin resistance (Chandola, Brunner \& Marmot, 2006), dyslipidemia (Biggi et al., 2008; Chandola et al., 2006; Karlsson et al., 2003; Seigrist, Peter, Cremer \& Siedell, 1997), high blood pressure (Fujino et al., 2006; Landsbergis et al., 2008; Vrijkotte et al., 2000), inflammation (Shetty, Economides, Horton, Mantzoros \& Veves, 2004), and obesity (Biggi et al., 2008; Brunner et al., 2007; Fujino et al., 2006; Karlsson et al., 2003). Work stress has also been associated with poor (Knudsen, Ducharme \& Roman, 2007) or impaired (Akerstedt et al., 2012; DeLange et al., 2009) sleep. Work stress may increase the likelihood of consuming an unhealthy diet (Crispim et al., 2011) or overeating (Payne, Jones \& Harris, 2005) and may also decrease the likelihood of exercise (Fransson et al., 2012; Kouvonen et al., 2005). Generally speaking, experiencing work stress has psychological and physiological consequences that are harmful to the health and wellbeing of the worker. In order to provide a comprehensive view of the work stress research, the literature review below was not limited to research with prediabetes as an independent variable. Rather, it includes research that addresses one or more of the variables known to be associated with prediabetes (socio-cultural factors, health behaviors and metabolic profile) and their association with work stress.

Two measures of work stress were included in this study. Concepts of work stress are based on models by Karasek and Siegrist. The Karasek Job Demand Control Model measures the effect of job strain on psychological and physical health outcomes (Karasek, 1979). Karasek defines job strain as a result of the interaction between job demands and decisional latitude, which have compounding effects (Karasek \& Theorell, 
1990). Level of job strain can be high or low. High job strain has detrimental effects on health outcomes (Karasek, 1979; Karasek \& Theorell, 1998). The Siegrist Effort-RewardImbalance Model measures the effect of job imbalance on psychological and physical health outcomes (Siegrist, 1996). Siegrist defines job imbalance as the discrepancy between effort and reward. Imbalance can be high or low. High job imbalance occurs when the worker's perceived effort is higher than rewards received. In other words, rewards that are perceived to be disproportionately lower than the effort put forth by the worker (below the level of expectation). Low job imbalance occurs when the worker's perceived effort and perceived rewards are low. Job imbalance (high or low) has negative effects on health outcomes (Siegrist, 2012). Each concept is described next.

\section{Job Demands}

Job demands are a component of Karasek's job strain. The term job demands refers to the workload placed on a worker. Job demands include physical and psychological work demands, time constraints, and conflicting demands (Karasek et al., 1985). Shift work and overtime are two examples of work demands. High job demands increase job strain. Shift work has been shown to increase the risk for impaired glucose metabolism (deBacquer et al., 2009; Karlsson et al., 2003; Suwazono et al., 2009), especially if working at night (Biggi et al., 2008; Toshihiro et al., 2008). Chronic overtime work has been linked to lack of participation in health-promoting behaviors, such as eating a healthy diet (Taris et al., 2011), which has been associated with an increased risk for prediabetes.

As stated previously, the relationship between job strain and prediabetes is not fully understood. However, multiple studies have shown that work hours and type of shift contribute to the prevalence of risk factors associated with impaired glucose metabolism. 
Day shiftwork, night shiftwork, swing shifts, and overtime have been shown to affect work performance and health negatively. Night shift workers have increased risk of prediabetes due to higher incidences of overweight, alcohol consumption, and overall stress (Biggi et al., 2008). When compared to high status day shift workers, day shift workers in low status jobs have evidence of altered glucose metabolism (Suwazono et al., 2009), hypertension, altered glucose, high lipids, and altered insulin metabolism (Karlsson et al., 2003).

Studies worldwide have demonstrated the effects of work hours on metabolic risk factors associated with impaired glucose metabolism. Using data from the Work, Lipids, and Fibrinogen Study (WOLF), Karlsson et al. (2003) investigated the relationship between shift work and metabolic indicators of cardiovascular disease and T2DM. Data were collected over one year. The participants $(n=1334)$ were Swedish men employed in one of two paper manufacturing plants as either day shift or rotating shift workers. Job duties were similar but not identical between the two types of shiftwork. Measures included anthropometric measurements, education, health habits data, lipid panel, blood pressure, fasting insulin, fasting glucose, and glycated hemoglobin. A modified version of Karasek's Job Content Questionnaire was used to measure job conditions. A single question measured social support. The data showed that mean serum insulin was higher in day shift workers compared with rotating shift workers ( $8.2 \mathrm{vs} .7 .5 \mathrm{mmol} / \mathrm{L}, p<0.05)$. Workers with rotating shiftwork were 1.43 times more likely to have high triglycerides (95\% CI, 1.13- 1.82) compared to their day shift counterparts, even after controlling for confounding variables (age, education, smoking, exercise, social support and job strain). Likewise, the rotation shift workers were 2.02 times more likely to have low HDL (95\% CI, 1.24 - 3.28). Odds ratios decreased slightly when adjusted for age and education (OR $=1.8195 \% \mathrm{CI}, 1.10-2.99)$, however, odds ratios increased when adjusted for low social 
support and high job strain $(\mathrm{OR}=2.03,95 \% \mathrm{CI}, 1.18-3.48)$. Rotating shift workers were also 1.34 (95\% CI, 1.07-1.69) times more likely to have high waist to hip ratio when compared to their day shift worker counterparts. The study used data from the WOLF population-based study. Population-based studies have robust methodologies that provide generalizable results, however, all of the participants were male, thus presenting a limitation to the findings. Results are limited to a Swedish male population. Results may differ in other European countries or other parts of the world. The study had other limitations. Although job duties were similar, day shift and rotating shift work participants were not matched. Results may differ with women or different workplace environments.

In a 14-year prospective cohort study by Suwazono et al. (2009), the researchers compared the effect of shift work on glycated hemoglobin in Japanese workers. The participants $(N=7104)$ were male day and alternating shift workers employed in a single steel company. Yearly measures included glycated hemoglobin, age, BMI, cholesterol, liver panel, and alcohol, smoking and exercise habits. Glycated hemoglobin incidence rates per 1,000 person years were sorted by percent increase as follows: 117.4 (10\% increase), 57.2 ( $\geq 15 \%$ increase), 26.9 ( $\geq 20 \%$ increase), 14.7 ( $\geq 25 \%$ increase), and 8.0 ( $\geq 30 \%$ increase). When compared to alternating shift workers, day shift workers had significantly higher glycated hemoglobin and BMI $(p<0.001)$. Percentage of participants who drank alcohol, smoked daily, or did not exercise was significantly higher in alternating shift workers than in day shift workers $(p<0.001)$. Type of shift was significantly associated with percent increase in glycated hemoglobin for each incidence rate except the $\geq 30 \%$ increase ( $p<0.02$ for all). The results showed that there was a dose-response relationship for length of time in alternating shift work and percentage increase in glycated hemoglobin for all incidence rates except for the $\geq 10 \%$ increase $(p<$ 
0.001). The researchers concluded that alternating shift work has a significant effect on glycated hemoglobin, and the effect is similar to established risk factors such as age and BMI. The strength of the study was the longitudinal design. However, the study did not include female participants. The alternating shift workers who participated in this study changed their shift an average of seven times during the 14-year cohort study. Results of this study may differ with female participants, workers in a different industry, or in persons with different types of work schedules. The results are further limited to a Japanese population.

In a study by deBacquer et al. (2009) a similar effect was noted. The researchers explored the relationship between shift work and the onset of metabolic disease. Using data from the BELSTRESS Study, a population-based, epidemiological, prospective study of Belgium industrial, male workers aged 35-59 years, the researchers analyzed baseline and follow-up data from 1,529 men working rotating or day shifts in nine companies. Metabolic variables included lipids, BP, BMI, waist circumference, and nonfasting blood glucose. Other measures included education, sleep quality, physical activity, and work conditions. Job strain was assessed using Karasek's Job Content Questionnaire. Type 2 DM diagnosis was based on self-report. Metabolic disease was confirmed if three of the five metabolic variables were high. When compared with day shift workers, the study identified that rotating shift workers, on average, were older, had lower educational level, and duties classified as blue collar with higher physical demands $(p<0.001)$. The incidence of metabolic disease was higher in rotating shift when compared to day workers $(\mathrm{OR}=1.77,95 \% \mathrm{CI}, 1.34-2.32)$. Reports of high job strain were similar between day and rotating shift workers. However, the data demonstrated a dose-response relationship between accumulating, rotating shift work and onset of metabolic disease $(\mathrm{OR}=1.46,95 \% \mathrm{CI}, 1.04-2.07)$, even after adjusting for all 
confounding variables. The researchers concluded that the incidence of metabolic disease appears to be causally linked to shift work, independent of socioeconomic, work, or lifestyle confounding variables. Although the researchers used a large sample derived from a population-based study, findings are generalizable to a worker population in Belgium. The study's participants were all middle-aged males. Results may differ in a female population, in varying age groups, and in another geographic region. The day shift start and end times were not reported. Participants were assigned to the rotating shift group if working a minimum of two different shift times per week. It is possible that different work schedules (start and stop times) or frequency and type of rotating shifts may yield different results.

Working strictly during the night also increases the prevalence of risk factors that are common to impaired glucose metabolism. Biggi et al. (2008) explored the relationship between night shift work and metabolic markers using a retrospective, longitudinal study. Participants included 488 Italian male, night workers aged 22-62 working as street cleaners and waste collectors. The comparison group included matched participants in the same job role but working in day shift positions. Participants whose work hours were from 1:30am to 7:30am were assigned the day shift group, while those working from 11:35pm to 5:35am were assigned to the night shift group. During the 31year period, the participants underwent medical examinations every two years to three years (day shift vs. night shift, respectively). The prevalence of high BMI (17.7 vs. 8.7\%) high glucose (5.1 vs. 3.8\%), high cholesterol (57.8 vs. $42.5 \%$ ), and high triglycerides (41.5 vs. $28.9 \%$ ) was higher in night shift workers when compared to day shift workers. There was a statistically significant difference in influence of night shift on BMI $(\mathrm{OR}=$ $1.18,95 \% \mathrm{CI},-0.10-45.0)$, smoking $(\mathrm{OR}=1.1,95 \% \mathrm{CI}, 0.9-1.3)$, alcohol use (OR = 1.1, 95\% CI, 0.9 -1.4), cholesterol (OR $=10.3,95 \% \mathrm{CI}, 5.5-15.0)$, and triglycerides 
$(\mathrm{OR}=18.4,95 \% \mathrm{CI}, 4.4-32.5)$ when compared to day shift workers ( $p$ not reported). There was also a slightly higher incidence of cardiovascular disease for night workers $(\mathrm{RR}=2.0,95 \% \mathrm{CI}, 0.4-9.4)$ when compared to day shift workers. The researchers also concluded that night workers have increased weight, incidence of obesity, and higher triglycerides and cholesterol when compared to their day shift counterparts, even after adjusting for confounding personal and work factors. Results of this study were limited to Italian male workers within the same industry and work schedule. It is possible that results would differ in female workers within the same industry, workers with a different work schedule, or those with a different type of job (e.g., administration). Results may also differ in another country or geographic region.

Working more than full-time (overtime) may also affect health. Taris et al. (2011) used data from the Study on Health at Work to assess the effect of overtime on adverse health outcomes. The Study on Health at Work used an online format to recruit participants from a 100,000-person pool that was representative of a Dutch population. Using a 2-year, prospective design with 649 participants in The Netherlands, the effects of overtime on two health indicators, subjective health and BMI. The participants were mostly male (84\%) and averaged 40.9 years of age. All data were collected via online survey. Overall health was measured by self-report. Overweight or weight gain was used as a proxy measure of sedentary lifestyle and unhealthy diet. Physical activity and diet were determined through questions based on the Dutch Health Council recommendations for exercise and healthy diet. Data were also collected for age, sex, education, smoking, alcohol use, profession, overtime hours, job demands, job control, and income. The data showed that high levels of overtime were significantly associated with low levels of healthy diet and exercise behaviors (standardized effect $-0.10, p<0.01$ ) and subjective health (standardized effect $-0.06, p<0.05$ ) over time. The researchers reported that the 
number of participants who consistently worked overtime was "relatively small" (Taris, 2011, p. 358), therefore, limiting generalizability of results. Results were also limited to a Dutch population as sample characteristics reflected the Dutch population and exercise and diet variables were based on Dutch recommendations. It is possible that results may differ in other countries, with a more diverse sample, or when using different work, exercise or diet criteria.

Job strain and its relationship to cardiovascular disease has been studied in the US (e.g., The Framingham Study), however, US studies focusing on job strain and its relationship to prediabetes are lacking. In addition, the relationship between job strain and prediabetes is not fully understood. Studies have shown that job demands, a component of job strain, may affect glucose metabolism. Empirical studies have shown that shift work or overtime (components of job demands) are potential risk factors for impaired glucose metabolism within conditions such as metabolic disease. The direct effects of job strain on prediabetes, especially within a US population, have not been fully explicated. This study will attempt to address this gap in the literature by assessing the relationship between job strain and prediabetes in a US sample.

Job strain is the interaction effect of job demands and decisional latitude. A review of the literature related to decisional latitude and its potential effect on prediabetes are discussed next.

\section{Decisional Latitude}

Decisional latitude is a component of Karasek's conceptualization of job strain (Karasek \& Theorell, 1998). Decisional latitude is sometimes called job control (van der Doef \& Maes, 1999). Decisional latitude includes the following components: (1) opportunity to learn, (2) creative opportunities, (3) variety in job duties, (4) level of job 
skill required, (5) opportunity for developing one's own abilities, (6) level of repetitive work, (7) opportunity to make decisions about work, (8) the freedom to tailor the execution of work, and (9) an opportunity to participate in the operational decisions about work (Karasek et al., 1985). Simply put, decisional latitude is the combined effect of job decision-making authority (the power and freedom to make decisions about work tasks) and skill discretion (the opportunity to use and develop skills on the job) (Karasek \& Theorell, 1990). Decisional latitude interacts with job demands to result in neutral, beneficial, or detrimental job strain. Decisional latitude, not job demands, has a stronger correlation with health due to the regulation of psychological impact of demands (Karasek \& Theorell, 1990). High job demands and low decisional latitude can increase the risk for injury and accidents and may interfere with a worker's ability to maintain a healthy lifestyle (Mirowsky \& Ross, 2007). Little is known about the direct effects of decisional latitude on health.

Using a simulation exercise, Ivengar and Chua (2011) interviewed 83 students from an eastern US university to investigate the relationship between decisional latitude and a worker's attitude toward leadership. Measures included the worker's decisional latitude and their perception of leadership's agreeableness and conscientiousness. The researchers hypothesized a curvilinear, u-shaped relationship between the degree of a worker's decisional latitude and the degree to which the worker perceives leadership as agreeable or conscientious, where moderate decisional latitude had the most effect on perception of leadership agreeableness and conscientiousness. Each participant read a vignette and answered questions regarding the degree of decisional latitude experienced by the worker in the story. The researchers found that increasing decisional latitude (DL) led to significantly increased perceptions of agreeableness $\left(F_{92,128}=6.24, p<0.01\right.$, partial $\eta^{2}=0.09$; low DL: $M=3.40, S D,=1.05$; moderate DL: $M=4.10, S D=1.20$; high DL: $M$ 
$=4.24, S D=1.19)$. The increase in perceived agreeableness was significant for low and moderate DL $(p<0.05)$. Increasing DL led to significantly decreased perceptions of conscientiousness agreeableness $\left(F_{2,128}=6.65, p<0.01\right.$, partial $\eta^{2}=0.09$; low DL: $M=$ $6.07, S D,=0.93$; moderate DL: $M=6.10, S D=0.7$; high DL: $M=5.48, S D=1.08)$. The change in perceived conscientiousness was significant for moderate and high DL $(p<$ 0.01). The researchers then surveyed 115 (53\% male) MBA students' real-world interactions with managers in order to provide external validity to the results from the vignette scenarios. The participants had an average of 5.68 years of work experience $(S D$ = 2.71). Each participant completed a "leadership survey" (Ivengar \& Chua, 2011, p. 19). After identifying two previous managers (mostly male and upper management), the participants were asked to indicate leadership effectiveness, perceived agreeableness and conscientiousness, and decisional latitude. The results showed that DL had a positive, statistically significant relationship with perceived agreeableness $(\beta=0.38, S E=0.14, p$ $<0.01$ ), except at the highest level of DL. Decisional latitude had a negative, significant relationship with perceived conscientiousness $(\beta=0.40, S E=0.16, p<0.05)$ at all levels. The researchers concluded that, because moderate DL and its corresponding positive attitude toward leadership provided a more positive work experience, the worker had lower levels of stress. The study used simulated scenarios to determine the relationship between decisional latitude and attitude. It is impossible to know if the participants' conclusions would be the same in an actual situation with identical issues. The study then used "real-world validity" to substantiate the simulated results (p. 18). The participants were mid-level workers answering questions about upper level management (i.e., CEO, partner). Results of this portion of the study may differ in lower-level workers. All of the participants were undergraduate or graduate students from a single university. Results may differ within a current worker population. 
In a study by Rafferty, Friend, and Landsbergis (2001), the researchers investigated the direct and interactive relationship between job strain and burnout. Karasek's Job Demand Control Model was used to guide the concept of burnout. The participants included 164 workers (75\% female) living in New York who worked in direct human service "emotionally demanding" allied health occupations with mentallychallenged clients (p. 76). The researchers used the Job Content Questionnaire to assess job demands, control, and support. The Maslach Burnout Inventory assessed burnout. Burnout was defined as emotional exhaustion, depersonalization, or reduced personal accomplishment. The data showed that job demands were statistically significantly associated with emotional exhaustion $(r=0.52, p<0.001)$, higher depersonalization $(r=$ $0.27, p<0.001)$, but not personal accomplishment. The components of decisional latitude were reported separately. Lower skill discretion was statistically significantly associated with higher emotional exhaustion $(r=-0.28, p<0.001)$, higher depersonalization $(r=$ $0.22, p<0.01)$, and reduced personal accomplishment $(r=0.037, p<0.001)$. Decision authority, however, was only significantly associated with higher emotional exhaustion $(r$ $=0.17, p<0.05)$. After controlling for demographic variables and job demands, skill discretion explained a significant portion of variance associated with emotional exhaustion $\left(\Delta R^{2}=0.13\right)$, depersonalization $\left(\Delta R^{2}=0.08\right)$, and personal accomplishment $\left(\Delta \mathrm{R}^{2}=0.20\right)$. Workers with higher levels of depersonalization were White $(\beta=-0.28, p<$ $0.05)$ and younger $(\beta=-0.25 . p<0.05)$. Workers with reduced personal accomplishment were had lower skill discretion $(\beta=-0.40, p<0.01)$, were younger $(\beta=-0.24, p<0.05)$ or had less education $(\beta=-0.20, p<0.05)$. The researchers did not report assessment of the potential confounding effects of an "emotionally demanding" job role on burnout, such as experiences with or attitudes towards clients (p. 76). In other words, it is not possible to determine whether burnout was directly related to job strain. The effects of 
burnout on job strain may differ in a population of workers with less personally challenging job roles. The results may also differ in areas outside of New York City.

Using data from the BELSTRESS Study, Fransson et al. (2012) examined whether leisure-time physical inactivity was more common in workers with high strain or passive jobs when compared with workers in low strain jobs. The meta-analysis pooled data from 14 prospective studies across eight European countries. Job stress was measured using Karasek's Job Demand Control Model. Physical inactivity was assessed using self-report. The overall prevalence of physical inactivity for workers with high strain jobs was significantly higher compared to workers with low strain jobs $(23.9 \%$ vs. $18.6 \%$, respectively). The overall prevalence of physical inactivity for workers with passive jobs was $23.5 \%$ while the prevalence in workers with active jobs was $18.9 \%$. The results showed a significant association between job control (decisional latitude) and physical inactivity, even after adjusting for covariates. Odds for physical inactivity increased at lower levels of job control when compared to high levels of job control (OR $=1.2395 \% \mathrm{CI}, 1.15-1.31$ vs. $1.0595 \% \mathrm{CI}, 0.96-1.14$, respectively). The data from this large meta-analysis study can be generalized to a European population; the pooled sample included over 170,000 European men and women. However, results may differ in other countries. In addition, the data extracted from the 14 cohort studies to calculate the results were only part of the overall data within each study. The self-report questions related to physical activity were very similar but differed between studies. The work measures also varied slightly between studies. It is possible that like comparisons cannot be made between studies.

Decisional latitude is a component of job strain. Lower decisional latitude leads to higher job strain (Karasek \& Theorell, 1990). There is limited evidence to suggest that decisional latitude, as a single variable, is associated with an employee's experience of 
stress. However, there is some evidence of an interaction effect of job demands and decisional latitude (job strain) and its effect on risk factors associated with prediabetes. As with the job demands variable within job strain, neither the effects of decisional latitude nor direct effects of Karasek's job strain (job demands and decisional latitude interaction) on prediabetes have been fully explicated, especially within a US population. This study attempted to address this gap in the literature by providing evidence of the proposed relationship between job strain and prediabetes in a US sample. The interaction between job demands and decisional latitude is discussed next.

\section{INTERACTION BETWEEN JOB DEMANDS AND DECISIONAL LATITUDE}

Karasek's Job Demand Control Model illustrates how two critical aspects of work situations, job demands and decisional latitude (sometimes called job control) interact and result in job strain (Karasek \& Theorell, 1998). The interaction effect of job demands and decisional latitude has been shown to affect health behaviors. Using the Theory of Planned Behavior, Payne, Jones and Harris (2005) examined the impact of job strain on exercise and healthy eating behaviors. Participants included 331 men and women

employees of a single, large company in the United Kingdom (UK) working in managerial to administrative roles. Questionnaires assessed intention to exercise, intention to eat a healthy diet, perceived control, job demands, job control at baseline. Exercise and healthy eating behavior were assessed one week later via single-item survey. Although the largest predictor of exercise and healthy eating behavior was intention (25\% of variance), high job demands was found to significantly reduce perceived control over exercise behavior ( $8 \%$ variance). Low job demands, low job control, and passive jobs predicted an increased intention to consume junk foods (sweets and snack foods) (10\% variance). The purpose of the study was to determine the 
combined predictive validity of the Theory of Planned Behavior and Karasek's Job Demand Control Model and the effect of job strain on health behavior. Intent to exercise and eat a healthy diet was measured by a single-item survey. Although Karasek's Job Demand Control Model was selected to provide a framework for job strain, only the job demands scale of Karasek's Job Content Questionnaire (derived from the model) was used. Decisional latitude (job control), the second component of job strain, was measured with another scale. Therefore, job strain, as defined by Karasek, may not have been appropriately tested. The results are based on perception rather than action. Intent to exercise and eat healthfully was measured, not the behavior itself. Results may differ when measuring action rather than intent. Results are also limited to a UK population.

In the population based Finnish Public Sector Study, Kouvonen et al. (2007) measured the association between job strain and adverse health behaviors that lead to cardiovascular illness. The participants included 34,958 females and 8,154 males in10 towns. Measures of job strain were derived from the Job Content Questionnaire. Adverse health behavior measures included smoking and heavy drinking. Body mass index of $\geq$ 25 was used as a marker for poor eating behavior and physical inactivity. Participants were also asked to report the amount of time per week spent on leisure and commuting activities. Higher likelihood of adverse health behaviors (3-4 behaviors) was significantly more likely in women with low job control (OR 1.14, 95\% CI, 0.99 - 1.32), high job demands (OR 1.08, 95\% C,I 0.94 - 1.25), or high job strain (OR 1.34, 95\% CI, 0.99 1.58) ( $p<0.001$ for all), even after adjusting for age, education, marital status or job type. Similarly, higher likelihood of adverse health behaviors was significantly associated with low job control (OR 1.33, 95\% CI, 1.06 - 1.68) and high job strain (OR 1.43, 95\% CI, 1.11 - 1.85) but not with high job demands in men. High job strain was associated with higher smoking (OR 1.12, 95\% CI, 1.03 - 1.22), high BMI (OR 1.09, 95\% CI, 1.02 - 
1.17), and physical inactivity (OR 1.26, 95\% CI, 1.17 - 1.36) among women. High job strain was associated only with physical inactivity (OR 1.39, 95\% CI, 1.20 - 1.62) among men. The researchers concluded that women and men exposed to high job strain had a 1.3 to 1.4 increased risk of three or more adverse health behaviors when compared to their counterparts with low job strain. The researchers reported self-report of data to be the primary limitation of the study. Body mass index was used as a proxy for eating and exercising behavior. It is possible that a person may have healthy eating and exercising practices and maintains a high BMI (e.g., body building). The results are generalizable to a Finnish population and may vary in another geographic region.

In a previous study by Kouvonen et al. (2005), data from two Finnish cohort studies, the Finnish Public Sector Study and the Hospital Personnel Study were used to assess the association between job strain and leisure-time physical activity. The study included 46,573 primarily female (81\%) participants, which accounted for approximately one-fifth of the Finnish public sector employees. Questions from Karasek's Job Content Questionnaire were used to determine job strain. Volume of leisure-time physical activity was measured as a metabolic equivalent task index. A survey that asked about types of physical activity, level of intensity, and length of participation for each in hours per week. Other measures included demographic data, job role, smoking, use of alcohol, and anxiety. The data showed that employees with high job strain $(p<0.001)$ or passive job $(p<0.001)$ had significantly lower levels of mean time spent in leisure-time physical activity than employees with low strain jobs, even after adjusting for confounding variables. The researchers suggested that persons with high strain jobs may have less energy to engage in leisure-time physical activity after work and that passivity at work may lead to passivity at home, however, these hypotheses were not tested. The results of the study are limited by the cross-sectional design. They are also limited to a mostly 
female Finnish population. Findings may differ in another geographic region or with a more diverse participant sample.

In another population-based study, Brunner, Chandola and Marmot (2007) used data from the British Whitehall II study to examine the effect of job strain on degree of obesity. Participants included 6,895 men and 3,413 women aged 33-55 who completed data collection on at least four occasions over the 19-year study period. The researchers reported recruiting a primarily White European population. Job strain was measured using the Karasek's Job Content Questionnaire. Height, weight and waist circumference were measured during all phases of the study. Health behaviors, including smoking, alcohol intake, fruit and vegetable intake, and exercise were measured via survey. The results indicated that job strain was associated with higher odds of body mass obesity in men and women even in models adjusted for age, height, employment type, and health behaviors. Among women, chronic job strain ( $\geq 3$ exposures) was associated with higher odds of body mass obesity when compared to women with no job strain $(\mathrm{OR}=3.38,95 \%$ CI, $1.16-9.80, p=0.16$ ). Men who reported moderate job strain (2 episodes) had significantly greater odds of body mass obesity when compared to women with the same level of job strain $(1.70,95 \% \mathrm{CI}, 1.10-2.63$ vs. $0.53,95 \% \mathrm{CI}, 0.22-1.29, p=0.02)$. Men with chronic job strain had higher odds of waist obesity $(p<0.01)$. Perceived low social support was linearly associated with higher waist obesity for both sexes (trend $p<$ 0.01 for men and $p<0.05$ for women). The researchers concluded that incident obesity was related to reports of job strain in a dose-response manner. The results are limited to White European populations. It is possible that the results will differ with non-White European participants, non-European participants, and with a more diverse population. 


\section{Effort}

Siegrist defines job strain as an imbalance between effort and reward (Siegrist, 2012). As part of a social contract, an employee engages in work in anticipation of reciprocity. This engagement is defined as effort. Effort can be high or low, physical or psychological. Amount of effort applied towards work in anticipation of reward can be measured by determining perceived intensity of physical or psychological energy typically exerted at work (Siegrist et al., 2004). Effort is one-half of the ratio formula for calculating job imbalance. Siegrist model is relatively new (debut in 1994). Effort as a single variable and its relationship to health and, specifically, prediabetes has not been studied.

\section{Reward}

Siegrist (2012) defines reward as the result of three "transmitter systems" received by the worker (p. 2). The reward systems include: money (salary), esteem, and career opportunities, including job security. Rewards can be high or low. Rewards are one-half of the ratio formula for calculating job imbalance. Rewards as a variable and its relationship to health or prediabetes have not been studied. The interaction effect of effort and reward (imbalance) is discussed next.

\section{INTERACTION BETWEEN EFFORT AND REWARD: JOB IMBALANCE}

Siegrist's Effort-Reward-Imbalance Model shows how the reciprocal interaction between effort and reward results in job strain when the two concepts are out of balance (job imbalance). Job imbalance has been shown to affect overall health, cardiovascular health, and glucose metabolism. When developing the Effort-Reward-Imbalance Model, Siegrist (1994) conducted three Dutch epidemiological studies to determine effects of job imbalance on cardiovascular health. Two study designs were prospective while one was 
cross-sectional. One prospective study included male participants in a blue-collar job role. The other prospective study included male participants in a managerial role. The cross-sectional study also included male participants in a blue-collar role; all the participants were Chinese. All three studies concluded that the combination of workload (effort) with job insecurity (rewards) has significant, negative effects on cardiovascular health for middle-aged males. The conclusions are limited to males in a Dutch and Chinese population. However, other studies have had similar findings.

In a cross-sectional study by Siegrist, Peter, Cremer and Seidel (1997), the association between work stress (job imbalance) and two factors typical of cardiovascular disease was examined in a German population. The participants included 179 middleaged $(48.5 \pm 5)$ males working in a managerial role at a single automobile plant. Job imbalance was measured with the Siegrist's Effort-Reward-Imbalance Questionnaire (ERIQ). Cardiovascular variables included blood pressure, cholesterol, triglycerides, lowand high-density lipids, and plasma fibrinogen. The results showed that job imbalance (high effort and low reward) was associated with significant increases in low-density lipids $(\mathrm{OR}=3.57,95 \% \mathrm{CI}, 1.24-10.20, p \leq 0.05)$ and plasma fibrinogen $(\mathrm{OR}=6.71$, 95\% CI, $1.57-28.76, p \leq 0.01)$, indicating cardiovascular inflammation. Study findings were limited to German male employees in a single industry working in a specific job role (manager). It is possible that results would differ in females, another industry, different job roles, or in another geographic regions within and outside of Eastern Europe.

In a study by Xu et al. (2012), the association between job imbalance and glycated hemoglobin in a Chinese population was assessed. Data were derived from the population-based stress and health in Shenzhen (SHISO) worker study. Participants included 680 men and women (mean age 40) in various job roles, including blue-collar, 
managers, police officers, and teachers. A fasting blood sample from each participant measured fasting glucose and glycated hemoglobin. The Chinese version of Siegrist's ERIQ was given to each participant to complete independently. Higher glycated hemoglobin was statistically significantly associated with high effort or high job imbalance in men ( $p=0.001$ and $p=0.007$, respectively) when compared to men who did not report high effort or high job imbalance. However, the significance was lost after adjusting for confounders. Women with high effort and high job imbalance had significantly higher glycated hemoglobin when compared to women who did not report high effort or high job imbalance, even after controlling for age, BMI and exercise $(p<$ 0.001). Partial correlations showed that effort and job imbalance were statistically significantly associated with glycated hemoglobin $(r=0.22, p=0.21 ; r=0.21, p=0.006$, respectively). Reward was statistically significantly, negatively associated with glycated hemoglobin $(\mathrm{r}=-0.17, p=0.021)$. The researchers concluded that there is a genderspecific association between job imbalance and glycated hemoglobin. The study was based on population-based data for a Chinese population; therefore, results are limited to Chinese workers. The researchers did not report why the association between effort and job imbalance and glycated hemoglobin may have been lost after adjusting for confounders (age, BMI, exercise). It is possible that findings would differ in a geographic region outside of China or with diverse participants.

Li et al. (2013) examined the relationship between job imbalance on T2DM and prediabetes using a cross-sectional design. Data were derived from the German population-based study, the Mannheim Institute of Public Health Industrial Cohort Study. The study participants included 2,674 primarily male $(79.65 \%)$ participants from three industrial engineering and manufacturing plants in Southern Germany employed in a variety of job roles. Job imbalance was measured using Siegrist's ERIQ. Type 2 diabetes 
and prediabetes were confirmed through fasting blood glucose and glycated hemoglobin. The data indicated that a high effort-low reward ratio (high job imbalance imbalance) was significantly associated with T2DM and prediabetes in men, even after adjusting for confounders $(\mathrm{OR}=1.27,95 \% \mathrm{CI}, 1.02-1.58)$. When controlling for T2DM, the results showed that imbalance was related to a $25 \%$ higher odds ratio of prediabetes in men $(p<$ 0.004). The findings did not yield associations between job imbalance and T2DM or prediabetes in women. The researchers concluded that repeated or continuous activation of the stress response (as a result of work stress) might predispose employees to the development of T2DM. It is unclear if the relationships found between job imbalance and prediabetes in male participants would also be found in women; the in a sample had an unequal distribution between genders. The findings are limited to a mostly male, Southern German population. Repeating the study with a more diverse participant sample or in another geographic location may yield different results.

Limited empirical evidence suggests that work stress is related to physiological factors and health behaviors associated with prediabetes. What is unknown is the relationship between work stress (job strain or job imbalance) and prediabetes as an outcome variable. The interaction effect of work stress, socio-cultural factors, and health behaviors on prediabetes is also unknown. All of the studies have been conducted outside of the US. It is unclear whether the limited findings would be similar in the US. This study attempted to address this gap in the literature by exploring the relationship of work stress and prediabetes in a convenience sample of workers in a technical industry in central Texas. The relationship between health behaviors and prediabetes is discussed next. 


\section{HEALTH BEHAVIORS}

Physiological risk factors can predispose individuals to prediabetes. This predisposition for prediabetes can be intensified through a lack of participation in key health-promoting behaviors: healthy diet, regular exercise (ADAc, 2013; DPP, 2012; NIDDK; 2013), adequate sleep (Buxton, 2010; Knutson et al., 2006; Knutson et al., 2007), and refraining from alcohol (Cullman, Hilding \& Östenson, 2011) and smoking (Bajaj, 2012). Decades of research have shown that a poor diet and lack of exercise affect glucose metabolism through untoward changes in glucose tolerance or insulin sensitivity (ADA, 2013; DPP, 2012). The ADA nutritional guidelines position statement states that nutrition therapy can reduce the risk of prediabetes and T2DM in persons who are already at risk (ADA, 2008). The guidelines recommend a balanced, healthy diet that includes a reduction in overall calories, high fiber, low sugar, and low fat. The joint position statement from the American College of Sports Medicine (ACSM) and the ADA (Colberg et al., 2010) states that regular participation in exercise activity (150min/week) that includes aerobic activity, strength, and conditioning exercises can improve glucose tolerance and reduce the risk of $\mathrm{T} 2 \mathrm{DM}$ in all persons. In addition, The Diabetes Prevention Program (2012) study showed that moderate changes in diet and exercise improved glucose metabolism and were shown to prevent or delay progression to T2DM. Inadequate sleep ( $<6$ hours per night) has been linked to an increase in blood glucose, increased appetite, and preference for unhealthy foods (Buxton, 2010; Knutson et al., 2006; Knutson et al., 2007). Evidence suggests that high alcohol use may be associated with abnormal glucose metabolism (Cullman et al., 2011). Smoking has been associated with an increase in insulin resistance (Bajaj, 2012). Abnormal glucose metabolism and insulin resistance may predispose an individual to prediabetes. 
Dietary intake, participation in exercise activities, maintaining adequate sleep, use of alcohol and smoking are determined by individual choices and decision-making, therefore, these behaviors are modifiable. Involvement in health-promoting behaviors such as reduction of body weight, limiting carbohydrate intake, and exercising for 30-60 minutes per day on five days each week can improve impaired glucose metabolism and reduce the development of T2DM (DPP, 2012; DPPOSRG, 2013; Nathan et al., 2007) and, in some cases, return an individual's abnormal glucose levels to normal (Perreault et al., 2009). Reversal of sleep deprivation has been shown to reverse effects on glucose metabolism (Knutson et al., 2007). Use of alcohol and smoking are not essential to life, however, overuse (alcohol) and use (smoking) can lead to ill health. As modifiable risk factors, diet, exercise, sleep habits, smoking, and alcohol use may be adjusted to meet guidelines. Efforts to modify health behaviors include education, support, and counseling. Based on empirical studies, the health behaviors related to prediabetes are: diet, exercise, sleep, alcohol, and smoking. Literature to further describe the relationships between health behaviors and the development of prediabetes are presented next.

\section{Diet}

Prediabetes often begins with an excess intake of fatty acids in the diet derived from sugared soft drinks, refined grains, and processed meats, which increase fat storage, especially in the central region of the body (Buiso et al., 2007). The increased fatty deposits activate a pro-inflammatory response through adipocyte (fat cell) activation. The inflammation and adipocyte activation cause a decrease in glucose uptake and insulin sensitivity, changes linked with prediabetes (Buiso et al., 2007; Rao et al., 2004). These inflammatory mechanisms simultaneously affect blood vessels. This pro-inflammatory state may partially explain why persons with prediabetes have concurrent cardiovascular 
risk factors (Kanauchi et al., 2007). The ADA (2008) recommends a varied and balanced diet that includes fiber-rich foods, low sugar, and low fat to maintain normal glucose metabolism and healthy lipid profile. Overweight persons at risk for prediabetes or T2DM should achieve moderate reduction of body weight (7-10\%) through a balanced, reduced-calorie diet (ADA, 2008).

Research indicates that an unhealthy diet significantly increases the risk for prediabetes, its related inflammatory changes, and the subsequent development of T2DM (Antuna-Puente et al., 2008; Buiso et al., 2007; Rao et al., 2004). In a review by Bazzano, Serdula, and Liu (2005), the authors cited multiple population studies including the Nurses Health Study and the Iowa Women's Health Study. Based on their review, the authors reported data supporting the relationship between diet and development of T2DM. Across multiple studies, a diet that included a high intake of glucose or fructose significantly increased risk for T2DM. On the other hand, a diet that included high fiber derived from whole grains was inversely associated with risk for T2DM (Bazzano et al., 2005). Empirical evidence supports these conclusions.

In a review by Psaltopoulou, Ilias and Alevizaki (2010), the researchers analyzed meta-analysis articles that reported an association between diet and prediabetes over a published 9-year period. The review states that an increase in whole grain intake by twoservings per day was associated with a dramatic reduction in T2DM risk $(21 \%, 95 \% \mathrm{CI}$, $13-28 \%$ ), even after adjusting for confounders. Frequent meat consumption increased the relative risk of T2DM by $1.17(95 \% \mathrm{CI}, 0.92-1.48)$ for those with low total meat intake, 1.21 (95\% CI, 1.07 - 1.38) for red meat intake, and 1.41 (95\% CI, 1.25 - 1.60) for processed meat intake. Low carbohydrate diets ( $45 \%$ or less of total caloric intake) were associated with lower glycated hemoglobin, lower fasting glucose and lower triglycerides (significance not reported). Based on analysis of 40 empiric articles, the researchers 
concluded that proper nutrition is a significant primary prevention intervention to reduce risk of T2DM. Similar results reported in experimental studies are discussed below.

In an animal study by Kumarasamy et al., (2011) the researchers aimed to determine if short-term calorie excess resulted in adipose deposition, whether systemic inflammation occurred, and the subsequent effects on blood glucose and blood pressure using genetically controlled rats. Rats who were fed a diet higher in calories and total fat (American diet) for 90 days had higher fasting blood glucose when compared with their standard diet counterparts ( 90.1 vs. $63.7 \mathrm{mg} / \mathrm{dL}$, respectively). Even higher blood glucose levels were seen for the rats fed the American diet after 172 days when compared to the rats fed their standard diet (102 vs. $87 \mathrm{mg} / \mathrm{dL}$, respectively). The rats that ate the American diet showed signs of inflammation, increased heart and kidney size (through post-mortem weight analysis), higher overall mean systolic and diastolic blood pressure, and a flattened variability in blood pressure. The researchers concluded that compared to the standard diet intake, the American diet that included caloric overloading with a diet that was consistently high in calories significantly increased adipocyte-related inflammation $(p<0.001)$, raised fasting serum glucose $(90.1 \mathrm{mg} / \mathrm{dL}$ vs. $63.7 \mathrm{mg} / \mathrm{dL}, p<$ $0.001)$, and reduced the body's ability to regulate blood pressure $(p<0.001)$. It is impossible to determine if the results could be replicated using human participants due to the invasive nature of the study parameters. However, by using genetically controlled rats, the researchers aimed to simulate the human response as closely as possible. The rat's physiological response to eating a rich diet that includes high fat, high calorie foods may be similar in humans.

Focusing on an all male sample, Rosmond, Holm and Bjorntorp (2000) compared the relationship between food-induced cortisol levels and measures of obesity and abnormal glucose. The study included 284 men all aged 51 living in Sweden with varying 
occupations. Cortisol was measured seven times during one working day in a natural setting. The participants with a pre-existing, abnormal cortisol response (as with chronic stress) displayed a pathological cortisol secretion pattern after food consumption $(p<$ 0.005). The results showed that for those men with an existing, abnormal cortisol pattern (estimated at $5-10 \%$ of the sample), food consumption, especially after lunch, was associated with abnormal metabolic measures. The abnormal cortisol response (low variability) after eating present in these men led to higher waist-to-hip ratios, high triglycerides, and insulin resistance ( $p<0.005$ for all variables). Findings suggest that, in persons experiencing stress at baseline (high cortisol), the stress response after eating leads to untoward metabolic changes. The study was limited by inclusion of only male participants within one age group (age $=51$ ) during a single day. The researchers reported that the number of participants with an abnormal cortisol curve could not be determined with absolute certainty due to the lack of control measures in a natural setting. Further studies should include male and female participants across all ages over a longer period of time. The study was also limited to a single city in Sweden. Results may vary in geographic regions and cultural settings outside of Sweden.

In a large, cross-sectional study by McNaughton et al. (2009), the researchers reported that higher diet quality was significantly associated with lower systolic and diastolic pressure, lower total cholesterol, and lower fasting plasma glucose. The participants included 7,441 men and women with normal glucose tolerance participating in the Australian Obesity and Lifestyle Study. Dietary intake and quality were assessed by a 13-item age- and sex-specific survey, the Australian Guide to Healthy Eating. A total score determined level of dietary quality. Dietary quality questions included frequency of intake of whole grain cereals, lean protein, low fat diary, and a variety of fruits and vegetables. Higher scores translated to a higher diet quality. Glucose was 
assessed using oral glucose tolerance testing. In women, the results showed that higher diet quality was significantly statistically associated with lower blood pressure, lower total cholesterol, lower fasting plasma glucose, lower fasting plasma insulin, and improved dyslipidemia ( $p<0.05$ for all). In addition, higher diet quality among women was inversely associated with risk for prediabetes (using fasting glucose), even after adjusting for age, education, health behaviors, BMI, and menopausal status $(p=0.03)$. Among men, high diet quality was inversely related to prevalence of T2DM $(\mathrm{OR}=0.38$, 95\% CI, $0.18-0.80)$, even after adjusting for age, education, health behaviors, and BMI. Although the study was based on population-level data and likely generalizable, it is uncertain whether the results are generalizable outside of northern Australia. Although dietary guidelines may be similar across nations, the dietary survey used in this study was specific to the Australian diet.

Using a randomized-controlled, parallel group, repeated-measures design, Solomon et al. (2010) investigated the effects of low-glycemic index or high-glycemic index diets combined with a supervised exercise program over a 12 -week period. The participants included twenty-two older men and women (age $66 \pm 1$ ) with a high BMI (34.4 \pm 0.8$)$ enrolled from The Cleveland Clinic, Ohio. After enrollment, all participants started a 60-minute, five day per week exercise program. Participants were also randomly assigned to either a low-glycemic or high-glycemic diet. An exercise physiologist supervised the exercise program. A registered dietician developed the diet plans and assessed compliance. Prediabetes was determined using two measures: insulin sensitivity via hyperinsulimic-euglycemic clamp and glucose tolerance via oral glucose tolerance test and blood C-peptide concentration. All participants had reductions in fasting glucose, triglycerides, and cholesterol. However, the participants in the high-glycemic diet intervention group had markers of beta-cell dysfunction through an exaggerated increase 
in glucose response and increase in insulin secretion $(p<0.05)$. In contrast, the participants in the low-glycemic diet intervention group showed an improved response to ingested glucose through less exaggerated increase in blood glucose and insulin $(p<$ 0.05). The findings suggest that although diet and exercise generally improved metabolic profiles, eating high-glycemic diet destroys pancreatic beta cell function. Although the study was randomized and controlled, the findings are limited to older (mean age 66) individuals with high BMI. Since all of the participants completed an exercise program, it is unclear if the results were due to type of diet or a combination of diet and exercise. Results may differ in a larger population with varying ages and lower BMI.

Two population studies are often cited to illustrate the relationship between diet and prediabetes, one from Finland and one from the US. In the Finnish Diabetes Prevention Study (Tuomilehto et al., 2001), 522 middle-aged (aged 55), overweight (BMI 25 or higher) men and women with impaired glucose tolerance were randomly assigned to lifestyle intervention or control group. Participants were recruited across five centers in Finland over a 5-year period. The participants in the control group were given general, written, educational materials regarding diet and exercise. The participants in the intervention group were given detailed, individual-based counseling on diet, exercise, and weight loss. On average, progression to T2DM was noted at a rate of $3 \%$ per year in the intervention group versus $6 \%$ per year in the control group (absolute incidence 32 cases per 1000 vs. 78 per 1000 person-years, respectively). The cumulative incidence of T2DM after two years was $6 \%(95 \%$ CI, 3 - 9\%) in the intervention group and 14\% (95\% CI, 10 - 19\%) in the control group. At four years, the intervention group had a cumulative T2DM incidence of $11 \%$ (95\% CI, 6 - 15\%), while the control group rose to $23 \%$ (95\% CI, $17-29 \%)$. The researchers concluded that the cumulative incidence of T2DM in the intervention group was $58 \%$ lower than in the control group (hazard ratio $0.4,95 \% \mathrm{CI}$, 
$0.3-0.7, p<0.001)$. Population studies provide data that are generalizable to the population. The results of this study are limited to the Finnish population. All of the participants had impaired glucose metabolism at baseline. Results may differ in persons with normal glucose metabolism. It is also possible that results would vary in individuals with normal BMI or in other age groups. The one-on-one counseling received throughout the intervention may have influenced results. Results may differ in a more natural setting, where individuals make their own choices.

The US Diabetes Prevention Program (DPP) (DPP, 2002; DPP, 2009) was a population-based, randomized clinical trial similar to the Finnish Study. The DPP was a large, multi-center trial that started in 2002 and continues currently. The aim of the study was to determine if lifestyle intervention could prevent or delay the onset of T2DM. Participants included 3,234 overweight, multi-ethnic (White, African American, Alaska Native, American Indian, Asian American, Hispanic/Latino, or Pacific Islander) men and women with impaired glucose metabolism across 27 centers in the US. Participants were randomly assigned to a control group, an intensive, lifestyle intervention group, or medication (Metformin) only group. The lifestyle intervention included diet, exercise, and behavior modification counseling aimed at reducing overall dietary fat and calories, participating in 150 minutes of exercise per week, and a 7\% weight loss (DPP, 2002). The initial findings showed that lifestyle intervention reduced risk of T2DM by 58\% (incidence 4.8 per 100 person years, 95\% CI, 4.1 - 5.7). The medication (Metformin) only group reduced their risk of T2DM by $34 \%$ (incidence 7.8 per 100 person years, $95 \% \mathrm{CI}$, 6.8 - 8.8). Lifestyle interventions were even more successful in older (age 60 and above) participants. For older participants, risk for T2DM was reduced by 71\% (DPP, 2009). Over time, participants in the lifestyle intervention group developed T2DM at a rate of $5 \%$ per year compared to $7.8 \%$ per year in the Metformin group, and an $11 \%$ per year 
rate for the control group (DPP, 2009). Further data collection is scheduled for 2014. Results from this population-based study can be generalized to the US. All of the participants had underlying, impaired glucose metabolism; results may vary in individuals with normal glucose metabolism at baseline. A structured program and oneon-one counseling was provided for the lifestyle intervention participants. Results may differ if individuals are expected to adhere to a diet and exercise intervention independently.

The association between dietary habits and risk for prediabetes is well known. The pathogenesis reports by Buiso et al., (2007) and Rao et al. (2004) outline the basic physiological relationship between diet and glucose metabolism across populations. Years of research have guided expert agency dietary recommendations. It can be assumed that expert agency recommendations, such as those from the ADA, are supported by multiple studies using rigorous methods. Independently, empirical studies, including large cohort studies and animal studies, have also supported the relationship between diet and prediabetes. Based on expert recommendations and empiric evidence, diet is a significant risk factor for prediabetes. Therefore, diet was included as a health-behavior variable in this study.

\section{Exercise}

As with diet, the link between exercise and prediabetes has been long studied and established. The muscle contraction achieved through exercise increases glucose uptake into muscles and mobilizes liver glycogen stores to maintain glucose homeostasis (Colberg et al., 2010). The joint position statement by the ACSM and ADA (Colberg et al., 2010) recommends moderate to vigorous exercise for 30 minutes on most of the days of the week (five of seven) or a total 2.5 hours per week as part of a lifestyle change to 
prevent T2DM. Studies show that exercise activities such as aerobic activity (walking or running) or strength and conditioning exercises (weight lifting) twice daily for 20 minutes if light to moderate effort, 10 minutes for strenuous effort, or five minutes for very strenuous effort can reduce risk of diabetes by $46 \%$ (Colberg et al., 2010). Participation in exercise has a linear dose-relationship with pancreatic beta cell function (Malin et al., 2013). In other words, beta cell function improves as exercise levels increase (the more an individual exercises, the better the pancreatic beta cells function). In those who already have prediabetes, weight loss intervention programs that include moderate exercise can reduce the progression to T2DM by 58\% (DPP, 2012), whereas medications, such as Metformin, reduce the risk of progression by only 25\% (Santaguida, 2005).

The ACE/ACCE Task Force (2008) lists sedentary lifestyle as a high risk factor for likelihood of developing diabetes. In a study that included a worldwide population, Lee et al. (2012) showed that physical inactivity accounts for $7 \%$ of T2DM disease burden. Using US NHANES 2003-2006 data, Healy et al. (2011) indicated that total sedentary time is associated with waist circumference, dyslipidemia, inflammation, and impaired glucose metabolism. However, including activity breaks in sedentary time has been shown to decrease waist circumference and lower BMI, triglycerides, and glucose levels (Healy et al., 2008). In a review by Adams (2013), multiple studies showed that as little as 15 minutes of high intensity exercise spread over a 2 -week period (as little as a couple of minutes per day) improved insulin sensitivity, even without changes in body weight.

Using skeletal muscle mass as a proxy for physical fitness, Srikanthan and Karlamangla (2011) assessed the association between muscle mass and insulin resistance and altered glucose metabolism (prediabetes) in a representative US sample derived from the US NHANES III dataset $(N=13,644)$. Skeletal muscle index was determined by 
calculating the ratio between skeletal muscle mass (bioelectric impedance calculation) and total body weight. Prediabetes was assessed through serum insulin, fasting glucose, and glycated hemoglobin. The results indicated that for every $10 \%$ increase in skeletal muscle index, there was a $23 \%$ reduction in relative risk of prediabetes (95\% CI, 11 - 33). The researchers concluded that skeletal muscle mass relative to total body weight was inversely related to risk for prediabetes $(p=0.0007)$. Although the data included a nationally representative sample and can be generalized to the US, the reasons for skeletal muscle differences were not reported. The increased skeletal muscle mass relative to total body weight can be assumed to be a result of physical activity (exercise) and general fitness, but cannot be confirmed. Larger muscles may be an individual trait. It is possible that the differences in skeletal muscle composition were the result of natural differences in genotype or phenotype.

In a cross-sectional study by Healy et al. (2007), the association of sedentary time, light-intensity exercise and moderate- to vigorous-intensity exercise and glucose measures were assessed as part of the Australian Diabetes, Obesity and Lifestyle Study. The study included 173 Australian men and women without current diabetes. Activity was measured using a uniaxial Actigraph accelerometer worn continuously for a period of seven days and supplemented by a diary of physical activity. Glucose was measured using fasting and 2-hour post-meal tests taken before and after the 7-day period. The data showed that sedentary time was significantly associated with high 2-hour glucose results, even after adjusting for confounders (sex, age, and time) (adjusted $r^{2}=0.13, p<0.001$ ). An increase in light-intensity and moderate- to vigorous-intensity exercise were associated with lower 2-hour glucose result (adjusted $r^{2}=0.11, p<0.001$ for both). All the associations remained significant even after adjusting for other factors, including family history and waist circumference. The association between moderate- to vigorous- 
intensity activity and 2-hour glucose persisted after physical activity diary information was excluded $(b=-1.07, p=0.008)$, which reduced the likelihood of inflating results with inaccurate diary entries. The researchers concluded that sedentary activity is detrimental to glucose metabolism, but increasing activity improves glucose metabolism. The study participants were derived from a larger, population-based study in Australia. Population studies use robust data collection techniques and results can be generalized to an Australian population. However, results may differ in other geographic regions of the world. Definitions of light to vigorous physical activity may also differ in other countries or regions.

When coupled with reduction in caloric intake, regular, moderate-intensity exercise prevents the development of T2DM (Buiso, Butterworth \& Linden, 2007; Santaguida, 2005). In a review by Buiso et al., (2007) the researchers reported that obesity, sedentary lifestyle, high calorie diet, and high fat diet were correlated with the development of insulin resistance. Across multiple studies, a reduction in energy intake and an increase in exercise have been inversely associated with the degree of insulin resistance. Healthy diet and regular exercise seem to have a synergistic effect.

Brekke et al. (2004) evaluated the short-term effect of a lifestyle intervention on insulin sensitivity, metabolic, and anthropometric measures in non-diabetic, first-degree relatives of persons with T2DM. The study included 77 men and women from Sweden. Participants were assigned to one of three intervention groups: current lifestyle, diet only intervention, and diet and exercise intervention. Diet information was collected using a Food Frequency Questionnaire. Exercise habits were assessed using the Leisure Time Physical Activity Interview. Data were collected at baseline and after 16 weeks. The participants in the diet and exercise intervention group lowered their body fat $(r=-0.42$, $p<0.01)$ and had a $13 \%$ reduction in their fasting insulin $(r=-0.52, p<0.01)$. The diet 
and exercise intervention participants also had lowered total cholesterol $(r=-0.51, p<$ $0.01)$, lowered triglycerides $(r=-0.52, p<0.01)$, and lowered low-density lipids $(r=-$ $0.53, p<0.01)$. The researchers concluded that moderate lifestyle modifications, including diet and exercise, significantly improved metabolic risk factors in persons at risk for T2DM without current prediabetes. The study was limited by its size $(N=77)$ and location (Sweden). The results are further limited to individuals with family history of T2DM in first-degree relatives. Results may differ with a larger sample, in a sample of participants without family history of T2DM, or outside of Sweden.

The expert agency statements regarding the association of exercise and prediabetes are based on population studies with rigorous methodology and results generalizable to the US (ADA, 2013; ACE/ACCE, 2010; Colberg et al., 2008; Healy et al., 2011). Lifestyle intervention studies have established that sedentary activity is associated with higher blood glucose (Healy et al., 2007) and even moderate increases in activity are beneficial to blood glucose levels in persons with (DPP, 2012; Santaguida, 2005) and without prediabetes (Buiso et al., 2007; Brekke et al., 2004; DPP, 2012; Healy et al., 2007). Based on expert recommendations and empirical evidence, exercise is an important health behavior risk factor for prediabetes due to its effect on development of prediabetes and its effect on progression to T2DM. Therefore, exercise was included as a health-behavior variable in this study.

\section{Sleep}

Insomnia affects one-third of the US population (Daley, Morin, LeBlanc, Gregoire, Savard, 2009). According to the ADA, the association between sleep and diabetes is still a new concept. The ADA has no formal recommendations regarding assessment of sleep patterns when screening for prediabetes or risk for T2DM. However, 
the ADA acknowledges that elevated glucose or untoward changes in insulin secretion due to changes in circadian rhythm occur when people experience temporary or chronic sleep deprivation (Gebel, 2011). During normal circumstances, sleep initiates two critical process associated with glucose metabolism. Glucose tolerance is reduced during sleep as energy demands and energy expenditure lowers, and appetite is suppressed due to the decreased energy demands during sleep. Acute total sleep loss or a reduction of normal sleep by two hours in one week increases the release of pro-inflammatory cytokines. The response to these pro-inflammatory cytokines is altered glucose metabolism through reduced glucose uptake and decreased insulin sensitivity. Sleep deprivation also alters the levels of appetite-related hormones, which increase hunger and lead to overeating while awake. Through an increase in glucose levels and residual changes in appetite, chronic sleep deprivation increases the propensity for prediabetes and eventually leads to T2DM (Buxton, 2010; Knutson et al., 2006; Knutson et al., 2007).

Multiple research studies have demonstrated the link between reduced sleep and changes associated with prediabetes, such as glucose intolerance, elevated blood glucose (Biggi et al., 2008; Buxton et al., 2010; Rafalson et al., 2012; Wu et al., 2012) and impaired glucose metabolism (Byberg et al., 2012; Gangwisch et al., 2007; Punjabi et al., 2004). For example, sleep deprivation may be inversely related to glycated hemoglobin, a diagnostic marker of impaired glucose metabolism (Byberg et al., 2012). Sleep deprivation may also be related to over-activation and fatigue of the pancreatic beta cells secondary to chronic over-activation of the sympathetic nervous system, coupled with reduced brain consumption of glucose (Gangwisch et al., 2007). Multiple, empirical studies have found an association between sleep and prediabetes.

Speigel, Leproult and VanCauter (1999) examined the effects of sleep debt on metabolic and endocrine function in 11 healthy men aged 18-27 over a 16-night period in 
a controlled setting. The length of time in bed was changed over the course of the study. During the first three nights, the participants spent eight hours in bed. The following six nights, they spent four hours in bed, and the last seven nights, the participants spent 12 hours in bed to recover from sleep debt and return to baseline. Sleeping during the day was not allowed. Wrist activity recordings verified compliance. Measurements included salivary cortisol, heart and pulse continuous monitoring, intravenous glucose tolerance tests, and hormone tests. Glucose utilization was 30\% lower in the participants with reduced sleep compared with participants after sleep recovery $(p=0.0005)$, a pattern similar to persons with T2DM diagnosis. The insulin response to glucose was also $30 \%$ lower in the participants with reduced sleep compared with participants after sleep recovery $(p<0.04)$. The participants with reduced sleep demonstrated a decreased ability to metabolize carbohydrates after breakfast, suggesting glucose intolerance similar to persons with prediabetes $(p=0.05)$. The researchers noted that the slower glucose clearance, lowered glucose effectiveness, and impaired glucose tolerance during the nights with reduced sleep are consistent with diagnostic criteria for prediabetes. In addition, sleep debt was associated with abnormal cortisol levels and patterns $(p<0.01)$. The study is limited by its small sample size and inclusion of only healthy men. A larger sample, a sample that includes women, or participants at various stages of health may show different results.

Similarly, in a study conducted by Buxton et al. (2010), the researchers aimed to discover whether sleep restriction in healthy subjects reduced insulin sensitivity. Twenty healthy young men with a mean age of 26.8 and mean BMI of 23.3 were subjected to a controlled sleep environment. Before the start of the study, participants slept at home for at least five days (mean 8.9 days) with 10-hours per night in bed to ensure a sleep-replete state (no sleep debt). The participants were then admitted to a hospital for 12 days. The 
first three nights, the participants slept for 10 hours per night. The next seven nights, participants slept for five hours per night. During the last night, the participants slept for 10 hours per night. Wrist activity monitors were used to calculate activity. All received a controlled diet. Metabolic measures included intravenous glucose tolerance and insulin tests; saliva and urine cortisol tests; euglycemic-hyperinsulinemic clamp procedures; and blood glucose and hormone tests. During the nights with sleep restriction (5 hours), the participants had an increase in cortisol $(p=0.48)$ and norepinephrine levels $(p=0.39)$, indicating stress. In addition, during the sleep restriction phase, 15 out of 19 participants had decreased insulin sensitivity and a decrease in glucose tolerance $(p=0.001)$. Both studies included healthy, young adult males with no previous medical history. The studies were conducted in large metropolitan areas in the northern US. It is possible that the results are limited to this population. Further studies should include a diverse sample of male and female participants with varying health history from differing US regions.

The effects of sleep deprivation on glucose metabolism with a younger group remain consistent with middle-aged adults. In a study by Nedeltcheva et al. (2009), two hypotheses regarding the effects of sleep deprivation were tested. The participants included 11 middle-aged men and women of normal weight who reported usual sleep duration of 7.6 hours per night on average. The researchers evaluated whether sleep restriction to 5.5 hours per night during a 14-day period resulted in reduced glucose tolerance, decreased insulin secretion, and decreased insulin sensitivity, and whether sleep restriction was also accompanied by changes in stress hormones. The participants completed two, 14-day periods of sedentary activity, liberal diet, and scheduled bedtimes of 5.5 or 8.5 hours in random order. Sleep was recorded polygraphically. The data showed that, when compared to participants who slept 8.5 hours, the participants restricted to the 5.5 hour sleep time had an increased 2-hour after meal glucose level (144 
\pm 25 vs. $132 \pm 36 \mathrm{mg} / \mathrm{dl}, p=0.006$, respectively $)$, increased circulating insulin $(0.023 \pm$ 0.005 vs. $0.020 \pm 0.005 \mathrm{mg} / \mathrm{dl}, p=0.039$, respectively), decreased insulin sensitivity (3.3 \pm 1.1 vs. $4.0 \pm 1.6 \mathrm{mg} / \mathrm{dl}, p=0.026$, respectively), and impaired glucose tolerance $(p=$ 0.042). The restricted sleep also resulted in a $25 \%$ increase in circulating catecholamines that are responsible for sympathetic nervous system activation through stress hormones, norepinephrine, cortisol, and epinephrine. Although the study included men and women, the sample size was small $(N=11)$ and limited to Chicago, Illinois. All the participants were healthy and lean. Results may differ in overweight or obese participants. Ethnicity was not reported. Further studies should include men and women with varying ethnicity, BMI, and health status. Chicago is a large, US metropolitan area, however, the sample size was small $(N=11)$ and not likely representative of the population at large. It is possible that results would differ with a larger Chicagoan sample, in a smaller city, or in a rural area in the US.

In a shorter, naturalistic longitudinal study, Chaput, Depres, Bouchard, Astrup, and Tremblay (2009) explored the association between sleep duration and T2DM or impaired glucose tolerance (IGT) using data from the Quebec Family Study. The participants included 276 men and women between 21-64 years of age. Self-report sleep data were collected at baseline and at a 6-year follow up. Sleep was categorized as short ( $<6$ hours/night), normal (7-8 hours/night), and long ( $>9$ hours/night). Presence of T2DM and impaired glucose tolerance was measured by oral glucose tolerance tests. Adults with an average of 7.8 hours of sleep per night were used as a reference. When compared to the reference, the researchers found that short sleepers ( $<6$ hours/night) had increased risk for the development of T2DM (adjusted RR $=2.78,95 \% \mathrm{CI}, 1.61-4.12$ ), even after controlling for BMI, waist circumference, and percent body fat $(\mathrm{RR}=2.42$, 95\% CI, 1.41 - 3.15). The longitudinal design and sample size are strengths of the study. 
Self-report data may not accurately time asleep. Results are limited the population of Quebec. Results may differ with more robust measures of sleep or with a population outside of Quebec, Canada.

The sleep studies have smaller sample sizes, used experimental designs, and the conclusions remained consistent over different geographic regions and sample sizes. The studies by Speigel el al. (1999) and Nedeltcheva et al. (2009) were conducted in Chicago, Illinois. The study by Buxton et al. (2010) was set in Boston, Massachusetts. The study by Chaput et al. (2009) took place in Québec City, Québec, Canada. Each study used experimental designs. Empiric evidence shows that sleep may have a significant relationship with prediabetes. Sleep affects appetite, hormone regulation, and glucose utilization. Therefore, sleep was included as a health behavior risk factor for prediabetes.

\section{Smoking and Alcohol}

Smoking is harmful and affects health regardless of quantity. There are no recommendations for safe levels of smoking. Smoking affects every organ in the body and can significantly affect health and predispose the user to multiple diseases and conditions (CDC, 2014). Alcohol has some health benefits when used in moderation (ADA, 2013; CDC, 2014). Alcohol is considered safe and beneficial if the following guidelines are followed: women do not consume more than one drink per day and men do not consume more than two drinks per day. Daily allotment cannot be combined into one day of use (NIH, 2014). Smoking and overuse of alcohol is known to affect general health and predispose the individual to disease (e.g., heart disease, cancer). The detrimental effects of smoking or overuse of alcohol on prediabetes or T2DM has been associated with how each can weaken overall constitution, immunity, and compromise 
health, which can increase the risk for more aggressive illness (ADA, 2013; AHA, 2013; CDC, 2014). However, there is also evidence that each can have a more direct effect.

\section{Smoking}

The US Surgeon General's Report (USHHS, 2014) states that although there is insufficient evidence to infer that smoking is a direct cause of T2DM, the risk for developing T2DM is $30-40 \%$ higher for smokers than non-smokers $(\mathrm{RR}=1.37,95 \% \mathrm{CI}$, 1.31 - 1.44). Smoking also has a dose-response relationship T2DM. When compared with former smokers, current smokers had significantly more risk of T2DM (RR $=1.14,95 \%$ CI, 1.09 - 1.19). Light smokers $(\mathrm{RR}=1.25,95 \% \mathrm{CI}, 1.14-1.37)$ and heavy smokers (RR $=1.54,95 \% \mathrm{CI}, 1.40-1.68)$ had significantly more risk of T2DM when compared with non-smokers. The report states that across numerous studies in multiple countries, smoking has been associated with an increased risk for T2DM due to the development of insulin resistance and impaired glucose metabolism. Most of the studies included in the evidence report controlled for confounding variables such as weight gain, poor diet, physical inactivity, and high alcohol use. The overall recommendation by the US Surgeon General Report is that smoking is an critical, modifiable, behavioral risk factor for T2DM. The following small study is an example of the conclusions outlined by the US Surgeon General Report.

In an intervention study by Bergman et al. (2012), the researchers outlined how nicotine disrupts insulin sensitivity. The study included 12 healthy non-smokers and 10 smokers without active disease (T2DM, dyslipidemia, lung disease) of healthy weight (BMI between $20-25 \mathrm{~kg} / \mathrm{m}^{2}$ ). All participants were "college-aged" men and women with similar body size (p. 3158). Participants were prescribed a diet for three days prior to data collection. Measures included body composition, fasting glucose, lipid panel, stress 
hormones, muscle fiber composition (through biopsy), and number of cigarettes per day. Smokers reported one pack of cigarettes per day, on average. Smokers were then asked to participate in a smoking cessation intervention. Measures were taken at baseline and at after one and two weeks of smoking cessation. The results showed that triglycerides concentration was approximately twice as high in smokers when compared to nonsmokers $(p<0.0001)$. This relationship did not change after smoking cessation. Insulin sensitivity was significantly lower in smokers when compared with non-smokers $(p=$ $0.03)$. Insulin sensitivity significantly improved after smoking cessation in smokers $(p=$ 0.006). The researchers concluded that after 1-2 weeks of smoking cessation, insulin sensitivity improved in young smokers without changes in body weight. Although the sample size was small and limited to a young, lean population, the results are statistically and clinically significant. Smoking negatively impacted insulin sensitivity in healthy individuals. It is possible that the resulting ill health would be exaggerated in participants who are already unhealthy. Insulin sensitivity trended towards normal after just two weeks of smoking cessation in healthy individuals. Results may be slower in participants with poor health or overweight. The study should be replicated using a larger, more diverse sample.

\section{Alcohol}

Unlike smoking, drinking alcohol has both beneficial and detrimental effect on health (CDC, 2014; NIH, 2014). Some studies suggest a u-shaped relationship between alcohol use and T2DM. Health benefits are achieved when alcohol is used in moderation, however, non-drinkers and heavy drinkers have the highest risk of poor health (Djousse et al., 2007; Howard, Armsten \& Gourevitch, 2004). Heavy drinking consists of drinking eight or more drinks per week for women or 15 or more drinks per week for men. Heavy 
drinking disrupts glucose metabolism, fat metabolism, and promotes inflammation (Baik \& Shin, 2008), which are components of prediabetes and T2DM. Heavy drinking can also increase the risk of cardiovascular disease (CDC, 2014), which tends to co-occur with prediabetes. This complex relationship has been explored in various empirical studies.

In a review by Howard et al. (2004), the researchers assessed the effects of alcohol consumption on the epidemiology of T2DM. Thirty-two studies were included in the review. The results of the systematic review showed that, when compared to nondrinkers, individuals who drink 1-3 drinks per day (moderate drinkers) had a 33-56\% reduction in risk for T2DM. When compared to moderate drinkers, individuals who drank more than three drinks per day (heavy drinkers) had up to a $43 \%$ greater risk for T2DM. Similarly, the meta-analysis by Koppes et al. (2005) showed a u-shaped relationship between alcohol and risk of T2DM. Using 15 prospective, cohort studies, the researchers analyzed the strength and shape of the relationship between alcohol and risk for T2DM. Alcohol consumption was measured in grams per day. A standard drink in North America contains approximately 12 grams. The results indicated that the relative risk for T2DM was highest among individuals who drank less than 6 or more than 48 grams (less than half to more than 4 drinks) of alcohol per day $(\mathrm{RR}=0.88,95 \% \mathrm{CI}, 0.80-0.95$ and 0.93 , 95\% CI, $0.74-1.18$, respectively). The relative risk was lowest among individuals who drank 24-48 grams (2 to 4 drinks) of alcohol per day ( $R R=0.74,95 \%$ CI, $0.63-0.88)$. Like Howard et al. (2004), Koppes et al. (2005) concluded that moderate drinkers have approximately $30 \%$ lower risk of T2DM when compared to heavy or non-drinkers.

The study by Djousse et al. reported similar findings in an exclusively older group of participants. Using a prospective design, Djousse et al. (2007) examined the role of total alcohol consumption on the incidence of T2DM. The participants included 4,655 men and women, aged 65 and older, from four regions of the US. Data was collected 103 
every six months over a 7-year period. Measures included anthropometric data, health habits questions, cardiovascular screening, fasting blood glucose, oral glucose tolerance tests, and a survey detailing amount and type of alcohol consumed. The results indicated that, for men, the relative risk for T2DM was highest for non-drinkers and heavy drinkers (> 7 drinks per day) $(\mathrm{RR}=0.795 \% \mathrm{CI}, 0.4-0.1$ and 0.8, 95\% CI, $0.4-1.3$, respectively), while the relative risk for T2DM was lowest for moderate drinkers $(\mathrm{RR}=0.6,95 \% \mathrm{CI}$, 0.3 - 1.1), even after adjusting for age, BMI, education, and smoking. The trend for women was similar. The relative risk for T2DM was highest in non-drinkers $(R R=0.9$, 95\% CI, 0.5 - 1.4 for men and women combined), but the relative risk for T2DM among heavy drinkers and moderate drinkers was similar $(\mathrm{RR}=0.6,95 \% \mathrm{CI}, 0.4-0.9$ and 0.7 , 95\% CI, 0.4 1.0, respectively), even after adjusting for confounders. The researchers concluded that, overall, moderate use of alcohol (up to six drinks per week) was associated with a $40 \%$ lower risk of T2DM. Strengths of the study included its prospective design with a population-based sample. However, results can only be generalized to older, US adults. Most of the participants were non-Blacks (86\%). Results may differ among varying ethnicities or with a younger population. The amount of alcohol consumed did not indicate the pattern of use (e.g., daily vs. weekly). It is possible results would vary among binge drinkers.

In the prospective study by Baik and Shin (2008), the association between alcohol consumption and metabolic disease was explored. The participants included 3,833 male and female Koreans aged 40-69. Data was collected over a 4-year period. Measures included anthropometric data, health habits, lipid profile, blood pressure, fasting glucose, and drinking amount and pattern. Non-drinkers were used as the reference. Metabolic disease was confirmed when three of the following five factors were present: high waist circumference, high triglycerides, low HDL, high blood pressure or high blood glucose. 
Heavy drinking was associated with a $63 \%$ increased risk of metabolic disease $(\mathrm{RO}=$ $1.63,95 \% \mathrm{CI}, 1.02-2.62)$. Even after adjusting for confounders, heavy drinking also increased the relative odds for each component of the metabolic profile when compared to non-drinkers. Heavy drinking increased the risk for large waist circumference $(\mathrm{RO}=$ 3.61, 95\% CI, 1.09 - 12.01), high triglycerides $(\mathrm{RO}=1.60,95 \% \mathrm{CI}, 1.28-2.0)$, high blood pressure $(\mathrm{RO}=2.19,95 \% \mathrm{CI}, 1.7-2.82)$, and high glucose $(\mathrm{RO}=2.37,95 \% \mathrm{CI}$, 1.5 - 3.73). Low HDL was inversely associated with all levels of drinking $(\mathrm{RO}=0.68$, $95 \%$ CI, $0.54-0.87$ and. 0.46, for light vs. heavy drinkers, respectively 95\% CI, 0.27 0.78). When stratified by type of drink, each of the metabolic profile components was significantly associated with drinking liquor rather than with other types of liquor (e.g., wine). The researchers concluded that heavy drinking was significantly associated with risk of metabolic disease. The study was longitudinal, population-based with rigorous methodology, and can be generalized to a Korean population. Results may differ in populations outside of Korea or in participants less than age 40 . However, the following study had very similar results with a European population.

Using a prospective, cohort design Cullman, Hilding and Östenson (2012) investigated the association of alcohol consumption and type with prediabetes and T2DM. Participants included 5,128 Swedish men and women with normal glucose tolerance or prediabetes aged 35-56 as part of the Stockholm Diabetes Prevention Program. Half of the participants had a family history of T2DM. The study had three steps: baseline data collection, intervention program, and follow-up data collection 8-10 years later. Measures included glycated hemoglobin, fasting glucose, BMI, waist circumference, exercise, education, job strain, overall stress, and total alcohol use. Men in the high alcohol use group had significantly higher risk of prediabetes and T2DM than men in the low use group ( $\mathrm{OR}=1.42,95 \% \mathrm{CI}, 1.00-2.03, p<0.05)$, even after full 105 
adjustment with confounders (including family history). Risk for T2DM in women was lower when alcohol use low to occasional when compared high use $(\mathrm{OR}=0.44,95 \% \mathrm{CI}$, $0.20-0.98, p<0.05)$, however, the relationship was no longer significant after adjusting for confounders. The researchers concluded that risk of developing prediabetes or T2DM among alcohol drinkers was different between men and women. In addition, the women in the study reported to consume only one-third the amount of alcohol than the men. The design and measures are the strength of this study. Population based data can be generalized to the Swedish population. Results may vary in participants with different cultural or ethnic backgrounds or in regions outside of Sweden. Results may also vary in men and women with matched alcohol use patterns.

\section{SUMMARY OF HEALTH BEHAVIORS}

Behavioral risk factors are, by definition, amenable to change. Modifiable, behavioral risk factors associated with prediabetes are usually a consequence of lack of compliance with health behavior recommendations, such as those outlined. Expert recommendation and empirical evidence have confirmed a strong association between health-promoting behaviors, such as healthy diet, regular exercise (ADA, 2013c; DPP, 2012; NIDDK, 2013), adequate sleep (Buxton, 2010; Knutson et al., 2006; Knutson et al., 2007), abstinence from smoking (Bergman et al., 2012; USHHS, 2014), and moderate alcohol use (Cullmnan et al., 2011; Djousse et al., 2007; Howard et al., 2004) and prediabetes. Participation in health-promoting behaviors, such as eating a healthy diet (Atuna-Puente et al., 2008; Buiso et al., 2007; Rao et al., 2004) and exercising regularly (ADA, 2013a; NIDDK, 2013) regulates blood glucose, decreases insulin sensitivity, and reduces the risk for prediabetes (DPP, 2010; Perreault et al., 2009; Santaguida, 2005). When describing risk factors for prediabetes, it is important to consider modifiable, 
health-promoting behaviors that have been widely studied and well established. Identifying modifiable, health-promoting behaviors may provide the opportunity for individual education, support, and counseling to adhere to recommendations and the risk of prediabetes. Individual counseling can include strategies to reduce risk for prediabetes through effective coping. Coping is the personal and situational response to a stressor (Hutchinson, 2011). Coping can be a powerful tool and may help an individual at risk adopt positive health behaviors. Coping and its relationship to prediabetes are discussed next.

\section{Coping}

Coping is the effort to master the demands of stress and includes thoughts, feelings, and actions. Coping can be problem-focused, emotion-focused, or relational. Problem-focused coping is the individual's attempt to change a stressful situation by acting on the environment. Emotion-focused coping is a change in an individual's perception or interpretation of the stressful situation. Relational coping incorporates the needs of others into coping strategies. In other words, the response to a stressful situation is attenuated by other's needs (e.g., family). Coping is complex and dynamic. Coping can be personal or interpersonal. Both Karasek and Siegrist have incorporated the concept of coping in the measures of work stress. Karasek's Job Content Questionnaire includes questions regarding social support, individual control, and sense of team (Karasek \& Theorell, 1998). Siegrist Effort Reward Imbalance Questionnaire includes questions about support, respect, and fair treatment (Siegrist, 2012). Social support can assist individual with coping by providing interpersonal interactions and relationships that offset stressful feelings and situations (Hutchinson, 2011). For the purposes of this study, 
the focus of coping was limited to the relationship of coping with the concepts within the Conceptual Model of Metabolic Risk.

Astvik and Melin (2012) identified patterns of coping, distinguished differences among coping patterns, and analyzed the effects of coping strategies on health. Participants included 192 men and women (mean age 46.7, SD 11.0) working in one of nine public welfare offices in Sweden. Most of the participants were women (91\%). Measures included demographic data, employment characteristics, smoking, exercise, a survey of health indicators, a single question related to sleep, two surveys related to work conditions, and a 13-item coping survey based on a previous qualitative study. Results indicated three types of coping patterns: compensatory and quality-reducing, voice and support seeking, and self-supporting. The participants who used the compensatory and quality reducing pattern $(n=68)$ worked more intensely, took fewer breaks, worked more during off times, and thought about work more often. They also lowered demands for quality and asked supervisors to prioritize work tasks. The participants who used the voice and support seeking pattern $(n=80)$ actively asked co-workers and supervisors for help, asked for help in prioritizing work tasks, objected and voiced dissatisfaction when the workload was too heavy, and actively tried to find solutions. The self-supporting participants $(n=43)$ used fewer coping strategies when compared to the other groups. They did not use any of the coping patterns used by the other groups, did not prioritize work, did not ask for help, and did not object to excessive work demands. The data showed that compensatory and quality-reducing group worked despite illness $(64.7 \%)$ when compared to the other groups (32.9\% and $46.5 \%$, respectively) despite an equal number of days on sick leave $\left(x^{2}(2)=14.834, p=0.01\right)$. In addition, those in the compensatory and quality-reducing group had significantly more sleeping problems, tiredness, stress related symptoms, and were less satisfied with work ( $p \leq 0.001)$. 
Complex work demands were positively related to lack of recuperation $(\beta=0.27, p=$ $0.001)$. Social support was negatively associated to lack of recuperation $(\beta=-0.26, p=$ 0.001). The researchers concluded that there are three types of coping patterns. Although no direct relationship was found between coping patterns and indicators of health, it is possible that the compensatory and quality-reducing patterns of coping were associated with greater risk of health by working despite illness and exhibiting signs of stress. The study was relatively small and results cannot be generalized. Types of coping patterns had an unequal number of participants. Results may vary with male participants or with workers employed in another industry. Results are further limited to a Swedish population. Results may differ in another region or country.

In another study regarding coping and health, Fujunishi et al. (1998) examined the relationship between coping with stress and glucose tolerance abnormality. The study included 3,082 Japanese men and women aged 28-72 $(M=48.4, S D$ 8.7). Most of the participants were working (91.7\%). Stress and coping measures included: Stress and Coping Inventory, Dealing with Stress, Profile of Mood States, Ways of Coping Questionnaire, and Recent Life Changes survey. Glucose measures included oral glucose tolerance test and fasting blood glucose. The participants were then grouped based on the results of their glucose tests (Grade $1=$ normal, Grade $2=$ borderline [prediabetes], Grade $3=\mathrm{T} 2 \mathrm{DM}$ ). Based on the mean scores on the social support subscale of the Stress and Coping Inventory, utilization was significantly lower in the Grade 3 group when compared with the Grade 1 group $(p<0.05)$. Further analysis showed that utilization is independently and statistically significantly related to level of glucose abnormality $\left(F_{(2,598)}\right.$ $=2.65, p=0.007)$. Scores on existence and perception of social support were not significantly related to level of glucose abnormality. The researchers concluded that social support utilization is significantly related to glucose abnormality. The study had a 
large sample size and robust methodology. The study is 16 years old. Results may vary today. Results are also limited to a Japanese population. Results may vary in other regions outside of Japan.

Although empirical data are limited, coping as a potential contributor to the relationship between work stress and prediabetes is important to consider. Therefore, coping was included in this study.

\section{SUMMARY}

Prediabetes is a complex condition with subtle, often unnoticeable signs. The primary process during prediabetes is impaired glucose metabolism with degrading pancreatic beta cell function and simultaneous insulin resistance. With no intervention, prediabetes will progress to T2DM within 10 years (DPP, 2010). The risk factors for prediabetes are multi-factorial, including non-modifiable and modifiable aspects (ADA, 2013; Bloomgarden, 2008a; Garber et al., 2008). The various risk factors may have compounding and interactive effects. When considering risk factors for prediabetes within a worker population, evidence suggests that stress at work may have confounding effect on the development of prediabetes. The relationship of work stress and prediabetes as an outcome variable has not been fully explored. The interaction effect of work, sociocultural factors, and health behaviors on prediabetes is also unknown. Therefore, it is important to study whether work stress affects the occurrence of prediabetes. Identifying risk factors that are amenable to change may provide the opportunity to reverse the progression of prediabetes. This study attempted to address this gap in the literature by exploring the relationship between work stress (job strain and job imbalance) and prediabetes in a convenience sample of workers in a technical industry in central Texas. 


\section{Chapter Three}

\section{METHODS AND RESEARCH DESIGN}

This chapter describes the research design, setting, sample, data collection procedures, instrumentation, and methods for data analysis related to the research questions. A conceptual model, The Conceptual Model for Metabolic Risk, was developed for this study to frame the interrelated risk factors for prediabetes. Variables and the interactions between and among variables were drawn from a synthesis of screening and diagnostic criteria, treatment guidelines, and empirical data related to prediabetes, the outcome variable. The Conceptual Model for Metabolic Risk is situated within a contextual environment, the computer company.

The purposes of this dissertation study were to: 1) describe the prevalence of work stress (job strain and job imbalance) and prediabetes in full-time employees at the central Texas headquarters of a computer technology company, 2) describe the relationship between stress, work stress, and prediabetes, 3) explore the potential interactive effects of socio-cultural factors, stress, work stress, health behaviors, and coping on prediabetes, and 4) explore the potential mediating effects of health behaviors and coping on the relationship between work stress and prediabetes.

\section{RESEARCH DESIGN}

This exploratory study used a descriptive, cross-sectional design. The exploratory design provided a point prevalence description of prediabetes and work stress in individuals working in a single technical, industrial setting in central Texas. The design allowed for a cross-sectional description of the associations among socio-cultural factors, health behaviors, work stress, and the prevalence of prediabetes in full-time employees. Such a design was economical in terms of time and resources, but it was limited in its 
inability to make inferences over time (Polit \& Beck, 2012). A convenience sample of computer company employees was recruited to answer these questions:

1. What is the prevalence of prediabetes in full-time (FT) employees?

2. What is the prevalence of job strain in FT employees?

3. What is the prevalence of job imbalance in FT employees?

4. What is the relationship between job strain and prediabetes in FT employees?

5. What is the relationship between job imbalance and prediabetes in FT employees?

6. What is the relationship between job strain and job imbalance?

7. What is the relationship between job strain and stress?

8. What is the relationship between job imbalance and stress?

9. What combination of independent variables (socio-cultural factors, health behaviors, work stress) best predicts prediabetes?

10. Do health behaviors mediate the relationship between work stress and prediabetes?

11. Does coping mediate the relationship between either job strain or job imbalance and prediabetes?

\section{SeTting}

The setting for this study was the local campus of a large company headquartered in central Texas. The selected company is a computer technology company founded in the mid-1970s within the Professional, Scientific, and Technical Services sector. The Bureau of Labor and Statistics (2014) Professional, Scientific, and Technical Services sector designation is for companies that specialize in performing professional, scientific, and technical services for other companies, including development and production of computer systems and design. The computer company manufactures test and 
measurement hardware and software systems. This company employs more than 7,000 persons worldwide. The company headquarters is located in in central Texas. The Austin campus employs over 2000 mostly younger, male employees who work in technology, research, engineering, administrative, and managerial roles. In 2011, the computer technology company in central Texas conducted a mandatory, biometric screening of all employees and reported that more than half of their workforce was sedentary, overweight, or had high cholesterol. Nine percent of these employees were also reported to have elevated blood glucose (computer company, 2011). The mandatory biometric screenings have continued on a yearly basis since 2011. The current company annual report states that the prevalence of employees with elevated blood glucose has steadily increased year to year without a notable shift in employee demographics (computer company, 2014).

\section{SAMPLE}

The computer company (study site) conducts yearly, mandatory biometric screening exams for all employees. The population for this study included men and women aged 25 and older working full-time (32 or more hours/week) who completed a biometric screening exam and demonstrated an interest in participation in this dissertation study. A convenience sampling technique was used. Invitation to participate in this study was offered to full-time employees across all departments within the company. Recruitment strategies were designed to maximize participation from all departments in an attempt to approximate the company demographics.

\section{Inclusion Criteria}

The sample included employees who were willing to participate in this study and who met the following criteria: 
- $\quad$ Men or women aged 25 and older

- Working full-time

- $\quad$ Able to read English

- $\quad$ Gave informed consent via email

Every effort was made to include participants from differing backgrounds. No participants were excluded due to their race, gender, sexual orientation, ethnicity, or nationality.

The lower limit of age 25 was selected to include only adult-age participants. The latest neuroscience research has shown that the adolescent brain continues to mature and develop through the mid-twenties (Johnson, Blum \& Geid, 2009). It is reasonable to conclude that adulthood does not begin until age 25 . Health related decision-making and analysis may differ between a fully developed and under-developed brain. Therefore, only adults were included in this study.

\section{Exclusion Criteria}

Potential participants were excluded if they were pregnant, had a current T2DM or cardiovascular disease diagnosis (myocardial infarction, stroke). The focus of this study was to describe the relationships between study concepts with a prediabetes population. Pregnant women with elevated blood glucose who were diagnosed with gestational diabetes, which is unique and separate from prediabetes. Likewise, persons with current T2DM have progressed beyond prediabetes into a diabetes diagnosis. Most of the metabolic risk factors for cardiovascular disease are the same as with T2DM. As with T2DM, persons with a cardiovascular disease diagnosis have progressed past the point of metabolic risk into serious illness. 


\section{Sample Size}

The sample size for this study was calculated using the CDC StatCalc statistical program within EpiInfo (CDC, 2013). Epilnfo is a free data management system administered by the CDC for instrument design, data analysis, and sample calculation specifically designed for public health topics. The StatCalc tool within EpiInfo calculates study sample size based on the expected frequency of a disease or condition within a population, given a specific confidence level and design effect (CDC, 2013). Considering a prevalence of $35 \%$ for prediabetes among US adults (ADA, 2013), a confidence level of $90 \%$, and a design effect of 1.0 , the estimated sample size was 84 . Considering $40 \%$ of US workers report experiencing work stress (WHO, 2007), a confidence level of 90\%, and a design effect of 1.0 , the estimated sample size is 89 . The larger sample size calculation was chosen. In order to account for a rate of $25 \%$ missing or incomplete data, an additional 59 participants will be recruited. Therefore, a total of 111 participants were required for this study. The final participant sample was 133.

\section{Human Subject Protection}

Following approval from the Departmental Review Committee of The University of Texas at Austin (UT) School of Nursing, the UT Institutional Review Board (IRB), and the computer company human resources department, and the computer company legal department, participants were recruited by the PI and the computer company Health Center nurse during their visit to the Health Center for their mandatory, yearly biometric screening exam. During the biometric screening appointment, the principal investigator (PI) or the Health Center nurse asked every employee if they were interested in participating in this study. The employees who expressed interest in participating in this study were asked to provide their contact information (email address) on an index card. The PI collected the index cards at regular, scheduled intervals (at least weekly). The PI 
contacted all potential participants via email to provide information regarding the structure and process of the study, procedures to protect confidentiality and privacy, how to participate in or withdraw from the study, and the PI contact information. If individuals consented to participate they indicated their informed consent by clicking a secure hyperlink at the bottom of the email. Description and dissemination of study findings will be guided by the non-disclosure agreement between the computer company and the PI.

\section{RISKS AND BENEFITS}

This study was not expected to produce psychological, social, physical, economic, or legal harm to the participants that is any greater than they would ordinarily encounter in their daily lives. The potential risk for loss of confidentiality was reduced by deidentifying data and limiting access to participant data. Only the PI and supervising dissertation team had access to the participant's personal data (name and date of birth). Any hard copy information (index cards) was kept in a locked file cabinet in the primary investigator's office and will remain there for a period of five years after data analysis, at which time the hard copies will be shredded and securely destroyed. All electronic data was stored on the primary investigator's personal computer and secured by the use of double password protection and will remain securely stored for a period of five years after data analysis, at which time the electronic files will be deleted. No electronic data will be stored in a temporary device such as a jump drive. There were no direct benefits to the participants as a result of participation in this study. An indirect benefit to participating in this study was the satisfaction of helping the researcher collect data that may lead to an increased understanding of the relationships between job strain and the prevalence of prediabetes. 


\section{Procedures}

\section{Recruitment}

All employees at the computer company are required to complete a biometric screening exam each year. Per the request of the Health Center (HC) manager, recruitment for this study took place during the biometric screening exam appointment by the HC nurse and the PI. Flyers were placed in the HC lobby and exam room. The PI attended clinic twice weekly during biometric screening appointments (8am to 10:30am). During the biometric screening exam appointment, the PI or the HC nurse introduced the study and asked about interest in participating. Employees who expressed an interest in the study provided their contact information (name, email address) on an index card. The PI collected the index cards at least weekly. The PI then contacted the potential participants via email and provided general information about the study followed by details regarding the consent process, including consent to access their electronic medical record (EMR) for the 2015 biometric screening data. The PI was not allowed to access any EMR without explicit consent from the employee. The participants did not receive any incentives for participating in this study. The HC staff were informed of the purpose and process of the survey data collection but did not participate in the data collection procedures.

\section{Consent and Enrollment}

When the interested employees met inclusion criteria, they were sent an email that included a consent document and information regarding the study via Qualtrics, a secure online service, to an email address of their choice. The email explained the study, the consent process, the purpose and procedures of the study, how the findings would be 
used, the risks and benefits to their participation, how to withdraw from the study, and the PI's contact information.

Participants were encouraged to read through the materials before giving consent (Appendix A). Consent was obtained by clicking on a secure hyperlink located at the bottom of the email. After obtaining electronic consent, the participants were automatically enrolled in the study and continued on to a secure, online survey. No incentives were offered for participation in this study. A total of 256 email invitations were sent through Qualtrics to potential participants. Of the total, 169 potential participants (66\%) consented to participate and opened the Qualtrics survey via the hyperlink within the email. Eighty-four percent (133) of the surveys opened were completed.

The online survey began with questions regarding inclusion and exclusion criteria.

1. Are you 25 years of age or older?

2. Do you work full-time?

3. Can you read English?

4. Are you currently pregnant?

5. Have you been told that you have diabetes or gestational diabetes?

6. Have you been told that you have or have had a myocardial infarction (heart attack) or stroke?

If the participant answered "no" to questions 1-3 or "yes" to questions 4-6, a message appeared on screen thanking them for their interest and explaining why their further participation is not possible. If the participant answered "yes" to questions 1-3 and "no" to questions 4-6, the online survey continued to the demographic and socio-cultural 
questions, followed by scales to measure stress, coping, diet, exercise, sleep, and work stress.

\section{Data Collection}

Data collection for this study was two-fold: retrospective review of the participants' electronic medical records (EMR) containing the 2015 biometric screening exam data and a secure, online survey to collect remaining data. Biometric screening exam data were extracted from the EMR and included: height, weight, waist circumference, blood pressure, high-density lipids, triglycerides, and fasting blood glucose. The secure, online surveys collected demographic and socio-cultural data (age, sex, race/ethnicity, family history of diabetes in primary relatives, education, job role, and job hours), stress level (Perceived Stress Scale), coping (Brief Approach/Avoidance Coping Questionnaire), diet (Rapid Eating Assessment for Participants), exercise (Godin Leisure Time Exercise Questionnaire), sleep (Insomnia Severity Index), and work stress data (the Job Content Questionnaire and Effort Reward Imbalance Questionnaire). Permission to use all the instruments was obtained for this study. A summary of the seven instruments with reliability reported in empirical literature can be found in Table 1. A summary of the instruments and their respective reliability coefficients for this study are presented in Chapter Four under "Descriptive Statistics for Study Variables." There were 129 questions included in the seven, online surveys. All data were collected one time

only. A review of the Qualtrics data report revealed that it took participants 12-35 minutes to complete the surveys. Some participants completed the surveys all at once; some logged in multiple times before completing all surveys. Survey data were downloaded from the Qualtrics server to the PI's personal password-protected computer 
for analysis. The PI extracted the EMR data from the computer company server using a company computer.

Table 1. Summary of Instruments. $(N=133)$.

\begin{tabular}{|c|c|c|c|c|}
\hline Instrument & Variables & Subscales & Number of Items & Reliability \\
\hline Demographic & $\begin{array}{l}\text { age, sex, } \\
\text { race/ethnicity, } \\
\text { education, marital } \\
\text { status, family } \\
\text { history } \\
\end{array}$ & $\mathrm{N} / \mathrm{A}$ & 4 & $\mathrm{~N} / \mathrm{A}$ \\
\hline $\begin{array}{l}\text { Job Related Items } \\
\text { (required) }\end{array}$ & job role, job hours & $\mathrm{N} / \mathrm{A}$ & 2 & $\mathrm{~N} / \mathrm{A}$ \\
\hline $\begin{array}{l}\text { Rapid Eating } \\
\text { Assessment for } \\
\text { Participants } \\
\text { (REAP-S) }\end{array}$ & Diet & $\mathrm{N} / \mathrm{A}$ & $\begin{array}{l}16 \text { item } \\
\text { 3-point Likert }\end{array}$ & $\begin{array}{l}\text { Test-Retest } \\
\text { reliability } r=-.86\end{array}$ \\
\hline $\begin{array}{l}\text { Godin Leisure Time } \\
\text { Exercise } \\
\text { Questionnaire } \\
\text { (GLTEQ) }\end{array}$ & Exercise & $\mathrm{N} / \mathrm{A}$ & 2 & $\begin{array}{l}\text { Internal } \\
\text { Consistency } \\
r=0.74\end{array}$ \\
\hline $\begin{array}{l}\text { Insomnia Severity } \\
\text { Scale (ISI) }\end{array}$ & Sleep & $\mathrm{N} / \mathrm{A}$ & $\begin{array}{l}7 \text { item } \\
\text { 5-point Likert }\end{array}$ & $\begin{array}{l}\text { Internal } \\
\text { Consistency } \\
r=0.74 \\
\end{array}$ \\
\hline $\begin{array}{l}\text { Perceived Stress } \\
\text { Scale (PSS) }\end{array}$ & $\begin{array}{l}\text { Global \& Event } \\
\text { Specific Stress }\end{array}$ & $\mathrm{N} / \mathrm{A}$ & $\begin{array}{l}14 \text { item } \\
\text { 5-point Likert }\end{array}$ & $\begin{array}{l}\text { Internal } \\
\text { Consistency } \\
r=0.75 \\
\end{array}$ \\
\hline $\begin{array}{l}\text { Job Content } \\
\text { Questionnaire (JCQ) }\end{array}$ & Job Strain & 4 & $\begin{array}{l}71 \text { item } \\
\text { 4-point Likert }\end{array}$ & $\begin{array}{l}\text { Internal } \\
\text { Consistency } \\
r=0.74 \text { for men } \\
r=0.73 \text { for } \\
\text { women }\end{array}$ \\
\hline $\begin{array}{l}\text { Effort Reward } \\
\text { Imbalance (ERIQ) }\end{array}$ & Job Imbalance & $\mathrm{N} / \mathrm{A}$ & $\begin{array}{l}16 \text { item } \\
\text { 4-point Likert }\end{array}$ & $\begin{array}{l}\text { Internal } \\
\text { Consistency } \\
r=0.80\end{array}$ \\
\hline $\begin{array}{l}\text { Brief } \\
\text { Approach/Avoidance } \\
\text { Coping } \\
\text { Questionnaire } \\
\text { (BACQ) }\end{array}$ & Coping & $\mathrm{N} / \mathrm{A}$ & $\begin{array}{l}20 \text { item } \\
5 \text {-point Likert }\end{array}$ & $\begin{array}{l}\text { Internal } \\
\text { Consistency } \\
r=0.68\end{array}$ \\
\hline
\end{tabular}

\section{Instrumentation}

Information regarding the instruments used to measure key concepts in the study (metabolic profile, socio-cultural factors, stress, work stress, health behaviors, coping, and prediabetes) is provided below. Description begins with the outcome variable, 
prediabetes, which is situated within the metabolic profile, i.e., fasting blood sugar $100-$ $126 \mathrm{mg} / \mathrm{dL}$.

\section{Prediabetes}

Prediabetes is the interim state between degrading glucose metabolism and diagnosis of T2DM. It is a period of impaired glucose metabolism before confirming a diagnosis of T2DM. The hallmark signs of prediabetes are impaired fasting glucose, impaired glucose tolerance, or both (Abdul-Ghani \& DeFronzo, 2009; Rao et al, 2004). For the purposes of this study, prediabetes was determined through fasting blood glucose. The fasting blood glucose range required for determination of prediabetes is 100 $126 \mathrm{mg} / \mathrm{dL}$. During the biometric health exam, fasting blood glucose was measured by taking a blood sample. If the blood glucose was elevated, the employee was asked to return for confirmation of the blood glucose result within the next week. During the follow-up appointment, blood glucose results were to be confirmed by taking a second fasting blood glucose blood sample and by taking a blood sample for glycated hemoglobin. However, none of the employees with initial elevated fasting blood glucose results returned for follow-up testing. The employees were fasting prior to and during each exam. The fasting blood glucose results were thus extracted from the EMR. Only one result was required for confirmation of prediabetes (ADA, 2013a). For the purposes of this study, prediabetes was treated as a binary and continuous variable. For the confirmed fasting glucose, the binary variable cut score was: results between 100 $126 \mathrm{mg} / \mathrm{dL}$ were scored as "yes" for prediabetes. Any value above or below this range were scored "no". Treating fasting glucose as a binary variable allowed for analysis of prediabetes versus no prediabetes. Results within the $100-126 \mathrm{mg} / \mathrm{dL}$ range were used as the continuous variable. Analysis of the confirmed fasting blood glucose as a continuous 
variable allowed for correlations to determine direction and degree of relationship with the independent variables (socio-cultural factors, stress, coping, health behaviors, and other metabolic profile factors).

\section{Metabolic Profile}

For the purposes of this study, the metabolic profile included the outcome variable, prediabetes (described previously), and elements known to commonly occur with prediabetes: large body size, dyslipidemia, and high blood pressure. The method used to measure each variable is described below along with a description of how the variable was categorized during analysis.

\section{Body Size}

Two measures of body size have been identified as most significant for prediabetes risk: body mass and waist circumference (ADA, 2013). The method used to measure each variable is described below along with a description of how the variable was categorized during analysis.

\section{Body Mass}

An individual's body mass index (BMI) was calculated from two anthropometric measures: height and weight. The HC nurse took both measurements as part of the yearly, mandatory biometric screening. Height measurements were taken using a stadiometer and reported in feet and inches. Weight measurements were taken using an electronic scale and reported in pounds and ounces. The height and weight data were extracted from the EMR and were used by the PI to calculate body mass index (BMI) using the standard formula of Centers for Disease Control and Prevention (CDC, 2011b):

$$
\mathrm{BMI}=\text { pounds } / \text { inches }^{2} \mathrm{X} 703
$$


Body mass index results were considered a risk factor for prediabetes based on CDC recommendations for overweight or obese. The CDC states that a BMI of 25-29.9 indicates overweight. A BMI of 30 or greater indicates obesity (CDC, 2011b). For the purposes of this study, the BMI calculation was treated as a binary and continuous variable. The binary variable was determined with a cut score of 25 . Participants scored a "yes" if BMI is 25 or greater and "no" if BMI is 24.99 or less. Treating BMI as a binary variable allowed for analysis of overweight versus normal weight. Analysis of BMI as a continuous variable allowed for correlations to determine direction and degree of relationship with the dependent variable (fasting blood glucose).

\section{Waist Circumference}

The HC nurse took waist circumference measurements in inches using a tape measure. The waist measurement was taken as recommended, at the narrowest point between the ribs and hips when viewed frontally (CDC, 2011). The waist measurement was used to determine centrally located adiposity, a risk factor for T2DM (ADA, 2013). Based on CDC guidelines (CDC, 2011), waist measurements for women greater than 35 inches or waist circumference for men greater than 40 inches were considered a risk factor for prediabetes (ADA, 2013). Waist circumference data were extracted from the EMR. For the purposes of this study, the waist circumference measure was treated as a binary and continuous variable. For binary analysis, female participants scored a "yes" when waist circumference was 35 inches or higher and "no" when waist circumference was 34.9 inches or less. Male participants scored a "yes" when waist circumference was 40 inches or higher and "no" when waist circumference was 39.9 inches or less. Using waist circumference as a binary variable allowed for analysis of central adiposity (high girth) versus normal abdominal girth. Analysis of waist circumference as a continuous 
variable allowed for correlations to determine direction and degree of relationship with the dependent variable (fasting blood glucose).

\section{Dyslipidemia}

Two lipids have been identified as most significant for prediabetes risk: highdensity lipids and triglycerides (ADA, 2013). The method used to measure each variable is described below along with a description of how the variable will be categorized during analysis.

\section{High-Density Lipids}

High-density lipids (HDL) are a densely packed lipoprotein. The function of HDLs is to remove low-density lipids and triglycerides by transporting them to the liver for removal. HDLs also maintain integrity and function of the lining of blood vessels. High-density lipids are primarily found in plant-based fats. Serum laboratory tests revealing low HDL indicate a diet with poor vegetable lipoprotein intake (American Association for Clinical Chemistry, 2013). The ADA recommends that overweight persons younger than 45 years of age with low high-density lipids $(<40 \mathrm{mg} / \mathrm{dL})$ should be tested for prediabetes (ADA, 2013a).

The HC nurse measured HDLs during the biometric screening exam. A blood sample taken once during the biometric screening exam measured the high-density lipids. The employees were fasting prior to and during their biometric exam. After collection, blood samples were sent to Quest Laboratory in Dallas, Texas for analysis. The results were then sent to the Cooper Institute in Dallas, Texas for further analysis. The highdensity lipids results were extracted from the EMR. For the purposes of this study, highdensity lipids were treated as a binary and continuous variable using the cut score recommended by the ADA (2013a). For binary analysis, participants with a high-density 
lipids result of $39 \mathrm{mg} / \mathrm{dL}$ or lower scored a "yes" indicating low high-density lipids. Participants with a high-density lipids result of $40 \mathrm{mg} / \mathrm{dL}$ or higher will score a "no" indicating normal or high high-density lipids. Treating HDL as a binary variable allowed for analysis of abnormal lipid metabolism versus normal lipid metabolism. Analysis of HDL as a continuous variable allowed for correlations to determine direction and degree of relationship with the dependent variables (fasting glucose and glycated hemoglobin).

\section{Triglycerides}

Triglycerides are made when excess food intake from fats and carbohydrates are converted into fatty acids (American Heart Association [AHA], 2013). The excess intake of fatty acids leads to storage of fat in adipose tissue in the form of triglycerides. If excess fat and carbohydrate intake continues, storage of triglycerides is also found in nonadipose tissues such as muscle, liver, and the pancreas (Buiso et al., 2007). The ADA recommends that overweight persons younger than 45 years of age with high triglycerides (>150mg/dL) should be tested for prediabetes (ADA, 2013a).

A blood sample taken once by the HC nurse during the biometric screening exam measured the triglycerides. The workers were fasting prior to and during their exam. After collection, blood samples were sent to Quest Laboratory in Dallas, Texas for initial analysis. The samples were then sent to the Cooper Institute in Dallas, Texas for verification of results. The triglyceride results were extracted from the EMR. For the purposes of this study, triglycerides were treated as a binary and continuous variable using the cut score recommended by the ADA (2013a). For the binary analysis, participants with a triglyceride result of $151 \mathrm{mg} / \mathrm{dL}$ or higher scored a "yes" indicating high triglycerides. Participants with a triglyceride result of $150 \mathrm{mg} / \mathrm{dL}$ higher scored a "no" indicating normal or low triglycerides. Treating triglycerides as a binary variable 
allowed for analysis of abnormal lipid metabolism versus normal lipid metabolism. Analysis of triglycerides as a continuous variable allowed for correlations to determine direction and degree of relationship with the dependent variables (fasting glucose and glycated hemoglobin).

\section{Blood Pressure}

Blood pressure is the force of blood against the arteries. The ADA recommends that overweight persons younger than 45 years of age get prediabetes screening if their blood pressure reading is greater than 120/80 during a health exam (ADA, 2013a). The $\mathrm{HC}$ nurse measured blood pressure during the biometric screening exam. The HC nurse measured the blood pressure using a sphygmomanometer once while the worker was in a seated position. The PI assured correct blood pressure measurement techniques by observation of $\mathrm{HC}$ nurse during the biometric exam. The blood pressure result was extracted from the EMR. For the purposes of this study, blood pressure was treated as a binary and continuous variable using the cut score recommended by the ADA (2013a). The American Heart Association defines high blood pressure as either a systolic blood pressure at or above $120 \mathrm{~mm} / \mathrm{Hg}$ or a diastolic blood pressure at or below $80 \mathrm{~mm} / \mathrm{Hg}$ (AHA, 2014). For the binary analysis, participants with either a systolic blood pressure of $120 \mathrm{~mm} / \mathrm{Hg}$ or diastolic blood pressure of $80 \mathrm{~mm} / \mathrm{Hg}$ or higher scored a "yes" indicating high blood pressure. Participants who do not meet either systolic nor diastolic blood pressure criteria $(119 \mathrm{~mm} / \mathrm{Hg}$ or lower for systolic blood pressure or 79 or lower for diastolic blood pressure) scored a "no" indicating normal or low blood pressure. Treating BP as a binary variable allowed for analysis of high BP versus normal BP. Analysis of $\mathrm{BP}$ as a continuous variable allowed for correlations to determine direction and degree of relationship with the dependent variables (fasting glucose and glycated hemoglobin). 


\section{Socio-cultural Aspects}

Socio-cultural factors included: age, sex, race/ethnicity, family history of T2DM, education, job role, and job hours. The method used to measure each variable is described below along with a description of how the variable will be categorized during analysis.

Age

Age is the numerical representation of time since birth. Age data were collected as part of the online, demographic survey. Age was also included in the participant EMR. Participants were asked to enter their age in years. Age was evaluated as a continuous variable to explore the relationship between age and other variables. It was also evaluated as a binary variable with a cut score of 45 to explore the fit of this sample with the national statistic rates of prediabetes in individuals older than age 45. Participants aged 45 and older scored as "yes" to age. Those younger than age 45 scored as "no".

\section{Sex}

Sex is the categorization of either male or female based on genotype. Sex data was collected on the online, demographic survey. Participants were asked to select male, female or other. An "other" category will be offered for those participants whose gender identity that conflicts with inherited physiology. Sex was analyzed as a binary variable, female yes/no or male yes/no. Due to the limited scope of this study, there will be option for "other." Due to the limited scope of this study, gender identity was not measured.

Sexual orientation is the categorization of sexual and romantic attraction. It refers to the sex of those to whom one is sexually and romantically attracted (American Psychological Association, 2011). General categories of sexual orientation include attraction to members of one's own sex (homosexual), attraction to members of the other 
sex (heterosexuals), and attraction to members of both sexes (bisexuals). Due to the limited scope of this study, sexual orientation was not measured.

\section{Race/Ethnicity}

Ethnicity is the categorization of persons based on phenotype. The major categories for race recommended for data collection by the National Institutes of Health Office of Management and Budget are: White, African American/Black, Asian/Pacific Islander, and American Indian/Alaskan Native (NIH, 2001). The National Institutes of Health recommends that data collection for ethnicity at a minimum include Hispanic/Latino or non-Hispanic/Latino. The ADA states that prediabetes is more likely in non-Whites. Ethnicity data was collected as part of the online, demographic survey. Participants were asked to choose "yes" or "no" to Hispanic/Latino ethnicity. Participants were also asked to choose the "yes/no" response to each race category. Participants were, by default, multi-racial if more than one race category was selected. For the purposes of this study, each race and ethnicity selection was categorized as a yes/no binary variable.

\section{Family History}

For the purposes of this study, family history was defined as significant, family medical history for T2DM for at least one first-degree relative. The ADA lists having at least one first-degree relative with diabetes as a risk factor for prediabetes and T2DM (ADA, 2013a). Family history data was collected as part of the online, demographic survey. Participants were asked to select yes or no when asked if any first-degree relative currently has or has ever been diagnosed with T2DM. The question included a description of first-degree relative for clarification. First-degree relatives include parents and siblings (US National Library of Medicine, 2014). For the purposes of this study, family history was treated as a binary variable, where "yes" indicated having one or more 
first-degree relatives with diabetes and "no" indicated no first-degree relatives with diabetes.

\section{Education}

For the purposes of this study, education was defined as the level of completed full-time education (high school, college). The five questions chosen to measure highest level of educational attainment were selected from the Bureau of Labor Statistics (BLS) Measures of Education and Training (US Department of Labor [USDOL], 2015). The BLS lists eight categories that reflect typical entry-level education for general employment. Categories range from no education to doctoral degree. The educational attainment categories sort data into distinct categories for interpretation of type of education needed for a specific job. These eight categories have been used to collect population-level data such as the American Community Survey and the Current Population Survey for decades (USDOL, 2015). Three categories were selected for this study that reflected typical entry-level education for Sector 130, computer systems design and related services, within the Professional, Scientific, and Technical Services: some college, bachelor's degree, and master's degree. High school diploma and no high school was added to account for untrained workers, such as custodial employees. Level of education has been shown to have a direct association with level of health in multiple studies (Baker et al., 2011; Cutler \& Lleras-Muney, 2007; Fonseca \& Zheng, 2011; Ross \& Mirowsky,1999). As such, level of education was included in this study. Education data were collected on the online, demographic survey. Participants were asked to the select highest level of education they had completed. For the purposes of this study, education was treated as an ordinal variable. 


\section{Job Role and Job Hours}

For the purposes of this study, job role was defined as the current job title. Participants were asked to select job title and select usual hours of work. The job title choices were derived from: 1) the BLS Classification System for industry employment (USDOL, 2015), and 2) data collected from interviews with the HC nurse practitioner and human resources director. Six typical computer systems design and related services jobs were included in the survey. Two additional roles, maintenance/custodial and other, were included to account for untrained workers and allow for free entry. Job role is included in this study due to empirical evidence that has shown its relationship to overall health. Job role has been identified as a distinct variable that contributes to health and wellness in multiple studies (Marmot et al., 1978).

The Job Content Questionnaire (JCQ) Users Guide instructs users to collect data regarding job hours. The six questions are required by the JCQ Users Guide (Pieper \& Schwartz, 1985) and were included in this study. The JCQ overall reliability is generally acceptable, has been translated into more than 20 languages, and construct validity has remained stable (Karasek et al., 1998). Reliability and validity of the JCQ is discussed in the Job Content Questionnaire section below. The JCQ Users Guide includes job hours due to empirical evidence that has linked job hours and health outcomes. Job hours including shift work and overtime have been directly associated with health and illness (deBacquer et al., 2009; Fujino et al, 2006; Karlsson et al., 2003; Taris et al., 2011). Job role and job hours were collected on the online, demographic survey. For the purposes of this study, job role and job hours were treated as nominal variables.

\section{Stress}

Stress is the physiological, psychological, or social response to any event that demands adaptive resources of the individual. Stress can be acute or chronic (Hutchinson, 130 
2011). For the purposes of this study, stress refers to the global and event-specific stress resulting from harm, threat, or challenges. Stress has long been associated with health. Chronic stress taxes an individual's biologic response and can lead to acute and chronic illness (Hutchinson, 2011). Work stress is a potential type of psychological stress is described below. Global and event-specific stress was assessed with the Perceived stress scale (PSS). Data regarding global and event-specific stress stress was collected through the PSS online survey and is described next.

\section{Perceived Stress Scale}

The PSS was used to measure global and event-specific stress. The PSS is an instrument designed to measure the "degree to which situations in one's life are appraised as stressful" (Cohen, Kamarck \& Mermelstein, 1983, p. 385). The PSS is not a diagnostic tool, rather it is meant to make comparisons within a sample. The PSS has been used extensively to measure stress perceptions worldwide and has been translated in over 10 languages. The PSS is a brief, easy-to-understand, 14-item, 5-point Likert scale $(0=$ never to 4 = very often) questionnaire that measures stress from ongoing situations, stress from life events, predicts health outcomes, and predicts changes in utilization of health services (Cohen et al., 1983). It is designed to measure how unpredictable, uncontrollable, and overloaded a respondent assesses his/her life (Cohen \& Williamson, 1988).

The PSS was designed for use in community samples with at least a junior high school education. The PSS reliability and validity was originally reported from data in three groups: two groups of college students and one community group. Reliability and validity were further tested in a large, US representative sample. The overall reliability of the PSS, using Cronbach's alpha coefficients, is acceptable across the three original 
groups ( $r=0.84, r=0.85, r=0.86$, respectively). The internal reliability of the PSS in the US sample $(\mathrm{N}=2,387)$, using Cronbach's alpha coefficients, is generally acceptable $(r=0.75)$ (Cohen \& Williamson, 1988). Validity was reported by correlating the PSS with comparative scales using data from the three original groups. The PSS was significantly correlated with Life-Event Scale scores from the three original groups $(r=$ $0.35, r=0.24, r=0.49, p<0.01$, respectively). Correlation with the Center for Epidemiologic Studies Depression Scale (CES-D) scores was also significant; however, only the two college groups are reported ( $r=0.76, r=0.65, p<0.001$, respectively). The PSS has no subscales. The PSS was scored by reversing the scores on positive items (items $4,5,6,7,9,10)$ then summing the scores across all 14 items. Higher scores indicate higher appraisal of stress. For this study, stress was treated as a continuous variable.

\section{Work Stress}

For the purposes of this study, work stress refers to the concepts within the Karasek and Siegrist models (Karasek, 1979; Siegrist, 1996). Karasek’s (1979) model of work stress is based on the concept of job strain. Job strain includes job demands and decisional latitude. Job strain is the interaction effect of physical and psychological job demands and decisional latitude, or control over work-related decisions (Karaesek, 1979; Karasek and Theorell, 1990; Karasek et al., 1981). High job strain is the result of excessive demands at work coupled with inadequate control over work duties (Schnall, Dobson \& Rosskam, 2009). High job strain has been shown to affect worker health and early mortality (Marmot et al., 1978; Karasek and Theorell, 1990; Schnall et al., 2009).

Siegrist's (1996) model of work stress is based on the concept of job imbalance. Job imbalance includes effort and reward. Job imbalance is the result of an imbalanced, 
interaction effect of the amount of effort at work and reward received in return. High effort-reward imbalance is the effect of high efforts paired with low reward (Siegrist, 2012). High imbalance has been reported to affect physical and psychological health in a negative way, including overall stress, depression, cardiovascular disease, and T2DM (Siegrist, 2012).

The four concepts derived from the Karasek and Seigrist models (job demands, decisional latitude, effort, and reward) were included as variables for work stress (Karasek, 1979; Siegrist, 1996). A description and the method used to measure each variable are described below. The corresponding survey instruments along with a description of how the variable was categorized during analysis are also described.

\section{Job Demands}

Job demands is the measurement of how hard a worker perceives their work to be, both physically and psychologically. General physical demands are related to speed and difficulty of workload, time pressure to complete work and amount of work. General psychological demands are related to concentration, work disruption, and performance. Job demands also includes the worker's assessment of the degree of role ambiguity they experience and the amount of organizational constraints placed on task completion (Karasek et al., 1998). Job demands was assessed as part of the Job Content Questionnaire (JCQ). The job demands subscale includes five questions. The reliability of the subscale acceptable $(r=0.75)$. The job demands subscale's discriminant and construct validity has been confirmed through comparative analysis with the Quality of Employment Survey and factor analysis, respectively. Data regarding job demands was collected through the JCQ online survey. The JCQ is further described below. 


\section{Decisional Latitude}

Decisional latitude is the operationalization of the authority to make decisions at work plus the opportunity to use and develop skills on the job (Schnall et al., 2009). Decisional latitude is the combined effect of decision authority and skill discretion. In other words, decisional latitude is a measure of the worker's ability to control assigned work activities through work decisions and individual control (Karasek \& Theorell, 1990). Decisional latitude was assessed as part of the JCQ and collected through the online survey. The decisional latitude subscale has a total of nine questions. The subscale questions include two smaller scales: skill discretion (six questions) and decision-making authority (three questions). The reliability of the subscale good ( $r=0.83)$. The decisional latitude subscale's discriminant and construct validity has been confirmed through comparative analysis with the Quality of Employment Survey and factor analysis, respectively. The JCQ is further described below.

\section{Job Content Questionnaire}

Karasek's Job Content Questionnaire (JCQ) was used to measure the job demands and decisional latitude components of job strain. The JCQ is an instrument designed to measure the "content" of the respondent's work in a general manner that has been used to assess an individual's exposure to job strain and to predict illness development in different work settings worldwide. It has been translated in more than 20 languages. The JCQ was originally constructed for the Framingham Offspring Study. The questionnaire has 49 items, a 4-point Likert scale (1=strongly disagree to $4=$ strongly agree) that is selfadministered and measures: work hours, skill discretion, decision authority, decisional latitude, psychological demands, physical demands, job insecurity, coworker support, and supervisor support (Karasek et al., 1981). The overall reliability of the JCQ is generally acceptable, with the overall average Cronbach's alpha coefficient for men $(r=0.74)$ and 
for women $(r=0.73)$ (Karasek et al., 1998). Discriminant validity of the instrument was assessed during development by comparing the JCQ constructs with the Quality of Employment Survey (Karasek et al., 1998). Factor analyses were used to determine construct validity of the JCQ. Analyses were three-fold, exploratory analysis using a correlation matrix, factor extraction using eigenvalues, and rotation varimax to interpret the findings. The JCQ's eight factors demonstrated consistency with theoretical assumptions of job strain, with $62.4 \%$ of the variance explained (deAraújo \& Karasek, 2008).

The JCQ has four subscales: job skill discretion, job decision-making authority, job demands, and job decisional latitude. Two subscales determine job strain: job demands and job decisional latitude. The job decisional latitude score is determined by the sum of two, equally weighted subscales job skill discretion and job decision-making authority. The job demands score is a composite of five items: excessive work, conflicting demands, insufficient time to complete work, working fast, and working hard. Reliabilities for the subscales are as follows: workload demands (physical and psychological demands) $r=0.75$; decisional latitude (skill discretion and organizational influence on decision-making) $r=0.83$; social support (supervisor and coworker support) $r=0.82$. (Landsbergis, Schnall, Schwartz, Warren \& Pickering, 1998). Factor analysis confirmed construct validity by identifying distinct factor loadings in each of the subscale categories (deAraújo \& Karasek, 2008). Internal consistency analyses showed significant variance explained by workload demands (80\%), decisional latitude (78\%), and social support $(71 \%)$.

Job strain is defined through two mechanisms of comparison: 1) a participant score above the sample median on the job demands sub-scale as well as a participant score below the sample median on the decision latitude scale and 2) a participant score 135 
above the national average on the job demands sub-scale as well as a participant score below the national average on the decision latitude scale. These methods can be used by individuals to compare their score on the JCQ with their within company counterparts or to a national mean. However, the job strain score is calculated with a ratio term where the job demands score is multiplied by two then divided by the decisional latitude score. This calculation is recommended by the JCQ User Guide for small or single company studies (Karasek, 1986). A score greater than one indicates job strain. A $z$-score can be calculated by subtracting the mean of the sample scores from the national mean then dividing the total by the national standard deviation. A $z$-score determines the magnitude of job strain.

For this study, job strain was measured through the ratio term calculation between job demands when multiplied by two then divided by the decision latitude score for each participant. This method is recommended for smaller studies (Karasek et al., 1998). Job strain was defined through the two methods of comparison stated above. Magnitude of job strain was measured through $z$-scores. For this study, job strain was treated as a binary variable. A score greater than one scored as "yes" for high job strain and a score less than one scored as "no" for high job strain as recommended by the Job Content Questionnaire and User Guide (Karasek, 1986).

\section{Effort}

Effort is part of the social contract of work. It is the perceived amount of physical and psychological effort put forth by the worker to complete work tasks. Physical effort includes physical demands, time pressure and amount of workload. Psychological effort includes perceived respect, support, job security, potential for promotion, and appropriate salary (Siegrist, 2012). Effort was assessed within the Effort Reward Imbalance Questionnaire. Data regarding effort was collected through online survey. 


\section{Reward}

Reward is the expected return from the effort spent by the worker. Reward is based on feelings of gratification or satisfaction derived from three systems: money, esteem, and security. Money includes amount of salary and the perceived match between effort and monetary compensation. Esteem includes fair treatment, respect from superiors, and the perceived match between the workers training or education and job status. Security includes employment status, potential for promotion job retention, and job stability, and opportunities for career advancement (Siegrist, 1994; Siegrist, 2012). Reward was assessed with the Effort Reward Imbalance Questionnaire, collected through the online survey.

\section{Effort Reward Imbalance Questionnaire}

Siegrist's Effort Reward Imbalance Questionnaire (ERIQ) was used measure the effort and reward components of job imbalance. The ERIQ is an instrument designed to measure the "societal structure" of the respondent's work (Siegrist, 1995, p. 228). The ERIQ has been used worldwide to assess an individual's exposure to job imbalance and the relationship between job imbalance and health outcomes in different work settings. The ERIQ is a 16 item, 4-point Likert scale (1=strongly disagree to $4=$ strongly agree) self-administered questionnaire that measures physical demands; time pressures; work load; co-worker and supervisor respect; peer and supervisor support; job security; potential for promotion; and monetary compensation (Siegrist \& Montano, 2014). The overall reliability of the ERIQ, using Cronbach's alpha coefficients, is generally acceptable within a German population of white- and blue-collar male workers $(r=0.80)$ (Siegrist \& Montano, 2014).

The ERIQ has two subscales: effort and reward. A third, optional scale, overcommitment, measures a pattern coping. The effort subscale measures the amount of 
demand within the work environment. The effort score is calculated by summing six items. Scores can range from 6-24. Higher scores mean higher effort. The reward subscale measures the three reward systems, money, esteem and security. The reward score is calculated by summing ten items. Scores can range from 10-40. Lower scores mean lower rewards. The overcommitment scale includes an additional six items, with scores ranging from 6-24. Higher scores mean higher overcommitment.

Job imbalance is calculated by an effort to reward ratio multiplied by a correction term $(I=k E / R)$. The correction term is calculated by dividing the number of reward items by the number of effort items. A ratio of one indicated a perfect balance between effort and reward. A ratio of $<1$ indicated low job imbalance. A ratio of $>1$ indicated high job imbalance. The score from the overcommitment scale is not needed for calculating job imbalance (Siegrist \& Montano, 2014) and was not included in this study.

Siegrist's (1995) reliability and validity analyses are derived from a summary of evidence from three population-based, epidemiological studies on work-related stress and cardiovascular risk of German middle-aged males. Reliabilities for the subscales are as follows: effort (physical and psychological) $r=0.80$ and reward (money, esteem, and security) $r=0.84$. The test-retest reliability is 0.80 for the effort scale and 0.84 for the reward scale. Exploratory and confirmatory factor analyses (convergent, discriminant, criterion) results confirmed the theoretical structure of the subscales. For this study, job imbalance was treated as a binary variable. A score less than one was scored as "yes" for low job imbalance and a score greater than one was scored as "yes" for high job imbalance. 


\section{Health Behaviors}

Health behaviors that have been indicated as influencing the risk for prediabetes include: diet, exercise, sleep, and smoking, and alcohol. The method used to measure each variable is described below along with a description of how the variable will be categorized during analysis.

\section{Diet}

For the purposes of this study, diet refers to selection or limitation of the amount and type of food a person eats in order to meet the dietary quality recommendations from the US Department of Agriculture (US Department of Agriculture, 2010). Dietary intake in excess of caloric needs leads to weight gain. Excess dietary intake of fats and sugars can increase the deposition of fat in the form of fatty acids (Buiso et al., 2007). Data regarding diet were evaluated by questions on the online survey using the shortened version of the Rapid Eating and Activity Assessment for Patients (REAP-S) survey. The REAP-S is designed to measure food intake reflecting dietary guidelines and policies within the US. The REAP-S questionnaire has 16 items, a 3-point Likert scale (usually/often, sometimes, and rarely/never) that is self-administered, and measures the intake frequency of: fiber, fruits, vegetables, calcium-containing products, lean meats, fat, sugar, snack, and soda (Gans et al., 2006). The American Heart Association recommends the REAP-S for use in busy, outpatient settings to assess dietary habits and develop interventions (Rao et al., 2011). The authors of the REAP-S recommend its use as a nutritional screening tool for persons with prediabetes (Segal-Isaacson, Wille-Rossett \& Gans, 2004). In a validation study with young medical students (mean age 24.2, SD=3.8) the REAP-S was correlated with the Food Frequency Questionnaire (Segal-Isaacson et al., 2004). The REAP-S survey showed high test-retest reliability $(\mathrm{r}=0.86, \mathrm{P}<.0001)$ in young medical students and practicing physicians. The REAP-S was also correlated with 
the Healthy Eating Index score $(r=0.49, P=0.0007)$, indicating a significant association with intake the macro- and micro-nutrients used for comparison (Gans et al., 2006). For the purposes of this study, diet was treated as a binary variable. Sometimes or usually meeting each of the dietary guidelines recommended by the USDA scored a "yes" and eating in excess of dietary guidelines scored a "no".

\section{Exercise}

For the purposes of this study, exercise refers to current participation in a program of exercise or bodily movement that enhances health as recommended by the US Department of Health and Human Services (US Department of Health and Human Services [USHHS], 2008). The ADA recommends exercising 30 minutes per day most of the days of the week (five of seven) (ADA, 2013a) or 150 minutes per week (USHHS, 2008). Data regarding exercise were assessed using the Godin Leisure Time Exercise Questionnaire (GLTEQ) via online survey. The GLTEQ is an instrument designed to measure usual leisure-time activities in a general manner in a community setting. The GLTEQ was originally constructed in Canada but has been tested in various settings. The self-administered questionnaire has four items. Each item asks the participant to recall the last seven days. The first three questions ask the participant to indicate the number of times (on average) each of the following activities were completed: strenuous exercise, moderate exercise, and mild exercise for a minimum of 15 minutes. Each category has a description and examples. The last question asks participants to indicate how often during the last 7-day period a leisure-time activity was engaged in long enough to increase the heart rate or begin sweating using a 4-point scale (Often, Sometimes, or Never/Rarely) (Godin \& Shepard, 1985). The reliability of the GLTEQ is moderate to high, with the overall average Cronbach's alpha coefficient of $r=0.74$ in a sample of 53 healthy US 
adults, $r=0.81$ in a tri-ethnic sample of 319 US students in $5^{\text {th }}, 8^{\text {th }}$, and $11^{\text {th }}$ grade, and $r$ $=0.82$ in a sample of US 28 males and 50 females aged 20-59. Validation studies using discriminant to classify maximum oxygen consumption in 163 males and 143 females aged 18-65 showed 69\% of items to be correctly classified (Godin \& Shepard, 1985).

Total scores on the GLTEQ range from 0-100+. Higher scores mean more exercise. Scores are calculated by first multiplying the frequency of exercise with number corresponding to the type of exercise (9=strenuous, $5=$ moderate, and $3=$ light) then summing the products for the first three questions (Godin \& Shepard, 1985). For the purposes of this study, exercise was treated as a binary variable. Using the 150 minute per week recommendation for exercise (USHHS, 2008), participants whose GLTEQ score is 30 or higher scored a "yes" and those whose GLTEQ score is 29 or less scored a "no".

\section{Sleep}

Sleep is a natural period of suspension of consciousness where rest and inactivity occur and the body restores energy levels. Lack of sleep is hypothesized to affect glucose metabolism by increasing fasting glucose, decreasing insulin sensitivity, and increasing propensity for weight gain (Knutson, Spiegel, Peney, \& Cauter, 2007). Sleep patterns were assessed with the Insomnia Severity Index (ISI). Data regarding sleep was collected through the ISI online survey.

\section{Insomnia Severity Index}

The ISI is a brief instrument designed to assess the severity of nighttime and daytime insomnia during approximately the last week (Morin, Belleville, Belanger \& Ivers, 2011). It is a 7-item, self-report questionnaire that assesses the nature, severity, and impact of insomnia on a 5-point Likert scale, with a score range of 0-28. The total score 
is interpreted as: 1) 0-7 no significant clinical insomnia, 2) 8-14 sub-threshold insomnia, 3) 15-21 moderate to severe clinical insomnia, and 4) $22-28$ severe clinical insomnia. The survey provides subjective data about sleep onset, maintenance, early awakening, sleep pattern satisfaction, sleep interference, impairment and distress (Bastien, Villieres \& Morin, 2001; Morin et al., 2011). The ISI has demonstrated content validity of $72 \%$ and an internal consistency of 0.74 in a sample of 145 US men and women with mean age 41.4 years (SD 13.1, range $17 \pm 82$ ) who participated in a study to evaluate insomnia (Bastein et al., 2001). The ISI has been used to measure the effects of sleep on glucose in population studies as well as smaller studies. Results of the ISI were treated as an ordinal variable.

\section{Smoking}

For the purposes of this study, smoking was defined as the use of tobacco via cigarettes. Smoking is not recommended for health; there is no amount of smoking that is regarded as safe (USHHS, 2014). Smoking data were collected as part of the online, demographic survey. Participants were asked to respond "yes" or "no" to the question, "Do you currently smoke?" If answered "yes," participants were asked to answer a follow-up question, "How many cigarettes do you smoke per day?" As per USHHS recommendations (2014), participants who smoke less than 10 cigarettes per day were categorized as "light smokers." Participants who smoke more than 10 cigarettes per day were categorized as "heavy smokers." Participants who report smoking 10-19 cigarettes per day were categorized as "moderate smokers." For the purposes of this study, smoking was treated as an ordinal variable. 


\section{Alcohol}

For the purposes of this study, alcohol was defined as consumption of any beverage containing alcohol (beer, wine, spirits). Light to moderate intake of alcohol (up to 6 drinks per week) has been positively associated with health. No alcohol and heavy intake of alcohol (> 6 per week) are negatively associated with health (NIAAA, 2014). Alcohol data were collected as part of the online demographic survey. The NIAAA recommends a minimum of three questions to capture patterns of alcohol use. Participants were asked to answer the three-question set of alcohol assessment as recommended by the NIAAA. For the purposes of this study, alcohol was treated as an ordinal variable.

\section{Coping}

Coping is effort to master the demands of stress. Efforts to manage stress include thoughts, feelings, and actions. For the purposes of this study, coping refers to the psychological approach of an individual to stress management. Coping has long been associated with health (Hutchinson, 2011). Coping was assessed with the Brief Approach/Avoidance Coping Questionnaire (BACQ). Data regarding coping were collected through the BACQ online survey.

\section{Brief Approach/Avoidance Coping Questionnaire}

The BACQ was used to measure coping. The BACQ is instrument designed to measure the approach-avoidance dichotomy. The BACQ is a 20-tiem, 5-point Likert scale ( $1=$ disagree completely to $5=$ agree completely) instrument to evaluate coping strategies in six categories. The BACQ is scored by totaling scores for each of the 20 items. Higher scores indicate higher use of approach-oriented coping strategies. Lower numbers indicated use of avoidant-oriented coping strategies. Reliability and validity was 
measured by comparing the BACQ to the COPE questionnaire in two samples: a sample of 93 patients (67 females, 26 males) in the waiting room of general practitioners in south-eastern Norway and a sample of 206 psychology and medical students (120 females, 86 males) attending the University of Oslo, Norway. Reliability correlations were weak but significant and varied from 0.34 to $0.57(p<0.001)$. Validation factor analyses were used in each sample. Factor analysis demonstrated an overall internal consistency $(r=0.68)$. For this study, coping was treated as a continuous variable.

\section{Data Analysis}

\section{Data Management and Cleaning}

The IBM Statistical Package for Social Sciences (SPSS) version 23.0 was used for data analysis. All data were stored and data output was calculated on an encrypted password-protected computer. Data were entered into IBM SPSS 23.0 and analyzed using descriptive, parametric, and non-parametric tests. All data extracted from the EMR and downloaded from the Qualtrics server were entered directly into the IBM SPSS 23.0 software program by the investigator. The one-tailed significance level was set at $p=$ 0.05. Correlation, logistic regression, and mediation analyses were appropriate for this study, as the goal of this exploratory study was to understand relationships and interrelationships between the independent variables and the dependent variable, describe significant predictors, and explore mediation effects (Nunnaly \& Bernstein, 1994).

\section{Data Cleaning}

Data were visually inspected for missing values after downloading the file from the Qualtrics server. Next, the amount of missing data was evaluated. Ideally, a data set should have as few missing values as possible; missing data can affect a study's conclusions and may reduce statistical power for hypothesis testing (Rubin, Witkiewitz, 
St. Andre \& Reilly, 2007). The data were assessed to determine the degree of missing values. When $5 \%$ or fewer values are missing and are missing at random, then the listwise deletion method can be used. A missing values analysis was used to explore patterns of missing values. A missing values analysis showed that none of the surveys had more than $5 \%$ of data missing. A listwise deletion technique was used to account for missing values.

A listwise deletion technique removes all data for variables that have one or more missing values (Nunnaly \& Bernstein, 1994). In this study, missing answers (values) within individual surveys in the dataset were substituted with a zero. The zero served as a placeholder for missing responses within surveys. When calculating survey scores, any survey that contained a zero as a response was excluded. As such, incomplete surveys were not in the final sample of scores. In other words, listwise deletion (complete-case analysis) was used to remove survey results for any survey that contained missing values (Nunnaly \& Bernstein, 1994). Histograms and z-scores were used to determine data distribution and outliers. Histograms did not indicate outliers. None of the data yielded extreme $z$-scores $(>3.29$ or $<-.329)$. A correlation matrix revealed that none of the data were highly intercorrelated (multicollinearity) (Tabachnick \& Fidell, 2012). All data had correlations less than 0.90 .

\section{Research question 1: What is the prevalence of prediabetes in full-time employees?}

Prevalence statistics were used to explore research question one. To calculate prevalence, the following values were needed: 1) the number of occurrences of the condition (prediabetes) during a specific time period and 2) the population total in which the condition occurred within the same time period. Prevalence was calculated by diving 
the number of occurrences of prediabetes by the size of the sample population. The result was expressed as a percentage. The formula is as follows:

$$
\text { Prevalence }=\frac{\text { number of cases of prediabetes }}{\text { total sample }} \times 100
$$

The frequency of employees who reported a fasting blood glucose level that indicates prediabetes was calculated. This was used as the numerator. The denominator was the total number of participants to determine the proportion of workers with prediabetes. This provided a prevalence rate of prediabetes within the convenience sample of employees who participated in the study.

\section{Research question number 2: What is the prevalence of job strain in full-time employees?}

Research question two, prevalence of job strain in full-time workers, was determined by using prevalence statistics. To calculate prevalence, the formula above was used. The number of participants with job strain using the JCQ scores was calculated. The result was compared to the total number of participants to determine the proportion of workers with job strain. This provided a prevalence rate of job strain within the convenience sample of employees who participated in the study.

\section{Research question number 3: What is the prevalence of job imbalance in full-time employees?}

Research question three, prevalence of job strain in full-time workers, was determined by using prevalence statistics. To calculate prevalence, the formula above was used. The number of participants with job imbalance using the ERI scores was compared to the total number of participants. The proportion provided a prevalence rate of job imbalance within the convenience sample of employees who participated in the study. 


\section{Research question number 4: What is the relationship between job strain and prediabetes in full-time employees?}

The relationship between job strain and prediabetes in full-time employees was assessed by Spearman's rho correlation coefficient (@) using the SPSS statistical program. The Spearman's rho is a non-parametric statistic that can be used when data violate parametric assumptions or when data are measured with an ordinal scale. The computation for Spearman's rho correlations yields an $r$-value that is compared to a probability distribution.

$$
r_{s}=1-\left[\frac{6 \sum D^{2}}{N^{3}-N}\right]
$$

Spearman's rho measures both the strength and direction of association between two variables. The relationship can be positive or negative and range from -0.1 to +0.1 . The Spearman's rho correlation test ranks data then applies Pearson's equation to those ranks. Spearman's rho assumptions were met: 1) data were ordinal and higher and 2) scatterplots showed a monotonic relationship between the two variables. Data for job strain and prediabetes were not normally distributed. Prediabetes is a dichotomous variable. Therefore, the Spearman's rho correlation coefficient was appropriate.

\section{Research question number 5: What is the relationship between job imbalance and prediabetes in full-time employees?}

The relationship between job imbalance and prediabetes in full-time employees was assessed by Spearman's rho correlation coefficient using the SPSS statistical program. The Spearman's rho is a non-parametric statistic that can be used when data violate parametric assumptions. Spearman's rho measures both strength and direction of association between two variables. The relationship can be positive or negative and range from -0.1 to +0.1 . The test ranks data then applies Pearson's equation to those ranks. Spearman's rho assumptions were met: 1) data were ordinal and higher and 2) 
scatterplots showed a monotonic relationship between the two variables. Data for job imbalance and prediabetes were not normally distributed. Prediabetes is a dichotomous variable. Therefore, the Spearman's rho correlation coefficient was appropriate.

\section{Research question number 6: What is the relationship between job strain and job imbalance?}

The relationship between job strain and job imbalance in full-time employees was assessed through correlations by computing the Spearman rho correlation statistic (Q). Correlations provide a single number to describe the degree of relationship between two continuous variables. The relationship can be positive or negative and range from -1.0 to +1.0. Data for job strain and job imbalance were not normally distributed. The test ranks data then applies Pearson's equation to those ranks. Spearman's rho assumptions were met: 1) data were ordinal and higher and 2) scatterplots showed a monotonic relationship between the two variables. Correlations are useful to measure the strength and directions of relationship between two variables (Nunnally \& Bernstein, 1994).

\section{Research question number 7: What is the relationship between job strain and stress?}

The relationship between job strain and stress was assessed through correlations by computing the Spearman Rho correlation statistic (@). The Spearman Rho correlation statistic was used to account for the skewness of the job strain data. The test ranks data then applies Pearson's equation to those ranks. Spearman's rho assumptions were met: 1)

data were ordinal and higher and 2) scatterplots showed a monotonic relationship between the two variables. 


\section{Research question number 8: What is the relationship between job imbalance and stress?}

The relationship between job imbalance and stress in full-time employees was assessed through correlations by computing and Spearman Rho correlation statistic (Q).

The Spearman Rho correlation statistic was used to account for the skewness of job imbalance. The test ranks data then applies Pearson's equation to those ranks. Spearman's rho assumptions were met: 1) data were ordinal and higher and 2) scatterplots showed a monotonic relationship between the two variables.

\section{Research question number 9: What combination of independent variables (socio- cultural factors, health behaviors, work stress) best predicts prediabetes?}

Levels of prediabetes are not supported by empirical data; therefore, the outcome variable must remain dichotomous. Multiple logistic regression was used to identify the relationships between significant independent (predictor) variables and the dependent (outcome) variable. Multiple logistic regression calculates the extent to which more than one predictor is related to a dichotomous outcome variable (prediabetes yes/no) and predicts likelihood of membership in categorical outcomes (Field, 2009). Logistic regression transforms categorical data using logarithmic transformation, which allows for expressing non-linear relationships in a linear way (Field, 2009). The resulting equation values vary between zero and one, where a value close to zero indicates that the outcome variable is unlikely to have occurred and a value close to one indicates that the outcome variable is very likely to have occurred.

$$
y=1 /(1+\exp (-\theta X))
$$

Multiple logistic regression has three assumptions: 1) the outcome variable must be discrete (dichotomous), 2) there are no outliers in the data (no z-scores within -3.29 and 3.29), and 3) there are no high intercorrelations (multicollinearity) (Tabachnick \& 
Fidell, 2012). All assumptions of multiple logistic regression were met within this study. The outcome variable (prediabetes) was dichotomous. Outliers were not present as indicated by $z$-scores. None of the predictors exhibited correlations greater than 0.90 .

Multiple logistic regression predicts probabilities which are estimated by fitting models based on the available predictors to the observed data. Model fit is assessed with the log-likelihood statistic. The log-likelihood statistic indicates how much information remains unexplained after model fit. Large log-likelihood statistic values indicate poorly fitted models, e.g., the larger the value of the log-likelihood statistic, the greater number of unexplained observations. The Wald statistic is used to assess the contribution of the predictors. The Wald statistic reports whether the $\beta$ coefficient for a predictor is significantly different from zero. A significant difference from zero indicates that the predictor is making a significant contribution to the prediction of the outcome variable, prediabetes (Field, 2009).

Multiple logistic regression results can be reported either as regression coefficients (e.g., $\beta_{0}, \beta_{1}, \beta_{2} \ldots$ ) or as the exponential function of the linearized regression equation (e.g., $\exp \left(\beta_{0}+\beta_{1} x_{1}+\beta_{1} x_{2} \ldots\right)$. The odds ratio allows for interpretation of the multiple logistic regression by indicating the change in odds resulting from a unit change (proportionate change) in the predictor. Interpretation is based on the amount and degree of change in the odds. If a value is greater than one, it can be concluded that as the predictor increases, the likelihood that the event happens increases. A value less than one indicates that as the predictor increases, the odds or likelihood of the outcome decrease (Field, 2009).

A backwards elimination method was used wherein all of the significant dependent variables were entered in one block then variables with little influence on the model fit are removed. In the backwards elimination method, strong predictors with high 
probability are selected. The resulting fit is comparable to that of the full model (Royston \& Sauerberi, 2009). In studies with small sample sizes, all selection procedures are unstable, a series of analyses by Royston and Sauerberi (2009) showed that backwards elimination methods increase computational intensiveness.

\section{Research question number 10: Do health behaviors mediate the relationship between work stress and prediabetes?}

The mediation effect of health behaviors on the relationship between job imbalance and prediabetes in full-time employees was assessed through a mediation analysis using structural equation modeling. A mediation model offers a mathematical explanation for how or why two variables are related when a mediating variable is hypothesized to mediate the relationship between the independent variable and the dependent variable (Fairchild \& McKinnon, 2009).

Structural equation modeling is a statistical methodology that uses a hypothesistesting approach that fits networks of constructs to data (Byrne, 2006). SEM methodology is a general and powerful multivariate technique that uses conceptual models, path diagrams, or linked regression-like equations to capture observable and unobservable (latent) constructs (Gunzler, Chen \& Zhang, 2013) or complex relationships among observed variables. A hypothesized model can be tested statistically to impute relationships between unobserved constructs and observed variables. In situations where single paths link observed predictor variables with an observed response variable, the results are identical to those obtained with standard multiple linear regression. SEM models are represented by path diagrams (Gunzler et al., 2013).

Measurement error is a potential concern in mediation models. The SEM approach removes measurement error from the estimation of the relationships among the variables. SEM examines complicated models of mediation in a single analysis rather 
than testing separate regression analyses. SEM can show direct and indirect effects of mediation. In addition, the SEM methodology provides model fit of the hypothesized mediational model to the data.

\section{Research question number 11: Does coping mediate the relationship between either job strain or job imbalance and prediabetes?}

The effect of coping on the relationship between work stress imbalance and prediabetes in full-time employees was assessed through a mediation analysis using structural equation modeling.

The Table 2 below summarizes the concepts that are of interest in this study, the variables that make up each concept, the measurement level for each variable, and the source that was used to obtain data for each variable. 
Table 2. Concepts within the Conceptual Model for Metabolic Risk.

The table below describes: concepts with the Conceptual Model, variables within the concept, measurement type, and the data source used for data extraction.

\begin{tabular}{|llll|}
\hline Concept & Variable & Measurement & Data Source \\
\hline Metabolic Profile & Prediabetes & Continuous \& Binary & EMR \\
\hline & Body Size & Continuous \& Binary & EMR \\
\hline & Dyslipidemia & Continuous \& Binary & EMR \\
\hline BP & Continuous \& Binary & EMR \\
\hline & & & \\
\hline Socio-Cultural Aspects & Age & Continuous \& Binary & Online Survey \\
\hline & Sex & Binary & Online Survey \\
\hline & Race/Ethnicity & Nominal & Online Survey \\
\hline & Family History & Binary & Online Survey \\
\hline & Education & Ordinal & Online Survey \\
\hline & Job Role & Nominal & Online Survey \\
\hline & Job Hours & Nominal & Online Survey \\
\hline & & & \\
\hline Stress & Stress & Continuous & Online Survey \\
\hline & Work Stress & Continuous \& Binary & Online Survey \\
\hline & & & \\
\hline Health Behaviors & Diet & Binary & Online Survey \\
\hline & Exercise & Binary & Online Survey \\
\hline Sleep & Ordinal & Online Survey \\
\hline Smoking & Ordinal & Online Survey \\
\hline Alcohol & Ordinal & Online Survey \\
\hline Coping & Continuous & Online Survey \\
\hline Coping & & &
\end{tabular}

\section{SUMMARY}

The aim of this study was to describe the prevalence of work stress (job strain and job imbalance) and prediabetes in full-time employees at the central Texas headquarters of a computer technology company, to describe the relationship between stress, work stress, and prediabetes, and to explore the potential interactive effects of socio-cultural factors, stress, work stress, health behaviors, and coping, on prediabetes, and explore the potential mediating effects of health behaviors and coping on the relationship between 
work stress and prediabetes. Limited empirical data have shown a relationship between work stress and metabolic components of prediabetes, but none have shown a direct relationship with prediabetes as the sole outcome variable. There are no studies to date that have explored the relationship between work stress and prediabetes in a US population. Using a cross-sectional design this study addressed the gap in the science by describing the point prevalence description of work stress and prediabetes and exploring relationships between significant independent variables and prediabetes in full-time employees working in a technical industry in central Texas. In this chapter, the setting, sample, protocol and measures were described. 


\section{Chapter Four}

\section{FINDINGS}

This chapter describes the findings related to the research questions. The chapter is organized into three sections. The first section includes the demographic characteristics of the dissertation study participants. Descriptive statistics of the study variables, prediabetes, BMI, waist circumference, HDL, triglycerides, BP, stress, job strain, job imbalance, diet, exercise, sleep, smoking, alcohol, and coping in full-time employees at the central Texas headquarters of the computer technology company are included in the second section. The third section contains the findings from the data analyses that answer the research questions.

\section{Demographic Characteristics of Study Participants}

A total of 133 employees participated in the study. The participants were mostly male $(67.6 \%)$ with a mean age of 37.6 years (range $25-63, S D=10.33$ ). Seventy-nine percent of the participants described themselves as White. Twelve percent identified as Hispanic. The majority of the participants had a college education or higher $(88.5 \%)$. More than one-half of the participants were married (58.2\%). The participants primarily worked in engineering or technical roles $(69.5 \%)$. Over $93 \%$ of participants reported no previous medical history. None of the participants reported family history of T2DM. Seventy-two percent of participants were non-smokers. Most reported drinking alcohol (72\%) at least occasionally. Thirty-six percent of participants met USDA dietary guidelines. Most reported exercising sometimes or often $(75.37 \%)$, as recommended by the USHHS. Body size, measured by body mass index, ranged from underweight to obese. Almost one-half of the participants had a body mass index in the normal range $(48.8 \%)$, while more than one-half had a body mass index in the overweight or obese 
range $(50.5 \%)$. Ten of the participants $(7.9 \%, n=126)$ did not report job strain, as measured by the JCQ. One-third of the participants $(35.8 \%, n=123)$ did not have job imbalance, as measured by the ERI. A summary of the participants' demographic characteristics can be found in Table 3 .

Table 3. Demographic Characteristics of Participants.

$\mathrm{N}=133 . \%=$ percentage; $\mathrm{M}=$ mean of sample; $\mathrm{SD}=$ standard deviation of sample.

\begin{tabular}{|c|c|c|c|c|}
\hline Sex & $\%$ & $\mathrm{M}$ & SD & Range \\
\hline Male & 67.6 & & & \\
\hline Female & 32.4 & & & \\
\hline Age & & 37.16 & 10.29 & $25-63$ \\
\hline \multicolumn{5}{|l|}{ Race/Ethnicity } \\
\hline White & 79.4 & & & \\
\hline African American & 2.1 & & & \\
\hline Asian & 17.7 & & & \\
\hline Native American & 2.1 & & & \\
\hline Hispanic & 12.1 & & & \\
\hline \multicolumn{5}{|l|}{ Marital Status } \\
\hline Single/Never Married & 32.6 & & & \\
\hline Married & 58.2 & & & \\
\hline Divorced & 7.8 & & & \\
\hline \multicolumn{5}{|l|}{ Education } \\
\hline High School & 7.2 & & & \\
\hline Trade/Technical School & 4.3 & & & \\
\hline College/University & 61.2 & & & \\
\hline Graduate School & 27.3 & & & \\
\hline \multicolumn{5}{|l|}{ Job Type } \\
\hline Management & 13.8 & & & \\
\hline Office/Administration & 14.9 & & & \\
\hline Engineering/Technical & 69.5 & & & \\
\hline Job Hours & & 41.21 & 9.99 & $2-70$ \\
\hline
\end{tabular}

Data from seven scales plus biometric data derived from the participant's electronic medical record were included in this study. Data analysis in the following sections includes sample sizes that may differ from the total sample size $(N=133)$. Some of the participants chose not to answer questions within a scale or omitted a scale 
altogether. All survey responses were checked and double checked for correct values. Histograms and z-scores were used to determine distribution of data and inspected for extreme responses. Free text answers were converted to a numerical response when appropriate. For example, answering "every day" to frequency of exercise was converted to seven, indicating all days of the week. Missing values within a scale were accounted for during data cleaning by using listwise deletion. Final scale scores reflect the corrections made, if any. Sample sizes reported for each variable exclude incomplete scales. A summary of instruments with reliability reported in literature and reliability found within this study can be found on Table 4.

Table 4. Demographic Characteristics of Participants.

\begin{tabular}{|c|c|c|c|c|}
\hline Instrument & Variables & $\begin{array}{l}\text { Number of } \\
\text { Items }\end{array}$ & $\begin{array}{l}\text { Reported } \\
\text { Reliability }\end{array}$ & $\begin{array}{l}\text { Reliability in } \\
\text { this Study. } \\
\text { Cronbach's } \alpha\end{array}$ \\
\hline $\begin{array}{l}\text { Rapid Eating } \\
\text { Assessment for } \\
\text { Participants } \\
\text { (REAP-S) }\end{array}$ & Diet & $\begin{array}{l}16 \text { item } \\
\text { 3-point Likert }\end{array}$ & $\begin{array}{l}\text { Test-Retest } \\
\text { reliability } r=- \\
.86\end{array}$ & 0.71 \\
\hline $\begin{array}{l}\text { Godin Leisure Time } \\
\text { Exercise } \\
\text { Questionnaire } \\
\text { (GLTEQ) }\end{array}$ & Exercise & 2 & $\begin{array}{l}\text { Internal } \\
\text { Consistency } \\
r=0.74\end{array}$ & 0.32 \\
\hline $\begin{array}{l}\text { Insomnia Severity } \\
\text { Scale (ISI) }\end{array}$ & Sleep & $\begin{array}{l}7 \text { item } \\
\text { 5-point Likert }\end{array}$ & $\begin{array}{l}\text { Internal } \\
\text { Consistency } \\
r=0.74\end{array}$ & 0.89 \\
\hline $\begin{array}{l}\text { Perceived Stress } \\
\text { Scale (PSS) }\end{array}$ & $\begin{array}{l}\text { Global \& Event } \\
\text { Specific Stress }\end{array}$ & $\begin{array}{l}14 \text { item } \\
\text { 5-point Likert }\end{array}$ & $\begin{array}{l}\text { Internal } \\
\text { Consistency } \\
r=0.75\end{array}$ & 0.09 \\
\hline $\begin{array}{l}\text { Job Content } \\
\text { Questionnaire (JCQ) }\end{array}$ & Job Strain & $\begin{array}{l}71 \text { item } \\
4 \text { subscales } \\
\text { 4-point Likert }\end{array}$ & $\begin{array}{l}\text { Internal } \\
\text { Consistency } \\
r=0.74 \text { for } \\
\text { men } \\
r=0.73 \text { for } \\
\text { women }\end{array}$ & 0.71 \\
\hline $\begin{array}{l}\text { Effort Reward } \\
\text { Imbalance (ERIQ) }\end{array}$ & Job Imbalance & $\begin{array}{l}16 \text { item } \\
\text { 4-point Likert }\end{array}$ & $\begin{array}{l}\text { Internal } \\
\text { Consistency } \\
r=0.80\end{array}$ & 0.25 \\
\hline $\begin{array}{l}\text { Brief } \\
\text { Approach/Avoidance } \\
\text { Coping } \\
\text { Questionnaire } \\
\text { (BACQ) }\end{array}$ & Coping & $\begin{array}{l}20 \text { item } \\
\text { 5-point Likert }\end{array}$ & $\begin{array}{l}\text { Internal } \\
\text { Consistency } \\
r=0.68\end{array}$ & 0.70 \\
\hline
\end{tabular}




\section{DESCRIPTIVE STATISTICS FOR THE STUDY VARIABLES}

\section{Metabolic Profile Variables}

A summary of the metabolic profile variables can be found in Table 5. Prediabetes is determined when a fasting blood glucose result is elevated above the normal range ( $\geq 100 \mathrm{mg} / \mathrm{dL}$ ) but not within the Type 2 diabetes range ( $\geq 126 \mathrm{mg} / \mathrm{dL})$. A blood sample taken by the HC nurse during the employee biometric health exam measured fasting blood glucose. If the fasting blood glucose was elevated, the employee was to return for a confirmation of the result through a repeat blood sample for fasting blood glucose and a glycated hemoglobin test. None of the participants returned for a second blood sample to confirm the elevated blood glucose result and, therefore, confirm prediabetes. The computer company elected not to test for glycated hemoglobin this year; therefore, prediabetes was not confirmed with a glycated hemoglobin blood test. Seven participant EMR had missing fasting blood glucose levels. For the purpose of this analysis, prediabetes was determined if the fasting blood glucose reading documented in the EMR was at or above $100 \mathrm{mg} / \mathrm{dL}$ and below $127 \mathrm{mg} / \mathrm{dL}$. The mean fasting blood glucose in the sample of 126 participants with complete data was $89.9 \mathrm{mg} / \mathrm{dL}$ (range 61 $124 \mathrm{mg} / \mathrm{dL})$. The majority of participants did not have prediabetes $(80.9 \%)$. 
Table 5. Summary of Metabolic Variables.

\begin{tabular}{|c|c|c|c|c|}
\hline & $\%$ & $\mathrm{M}$ & SD & Range \\
\hline $\begin{array}{l}\text { Prediabetes }(\mathrm{FBG} \geq 100 \mathrm{mg} / \mathrm{dL}) \\
\text { Yes } \\
\text { No }\end{array}$ & $\begin{array}{l}19.1 \\
80.9\end{array}$ & & & \\
\hline $\begin{array}{l}\text { Body Mass Index } \\
\text { Underweight } \\
\text { Normal } \\
\text { Overweight } \\
\text { Obese }\end{array}$ & $\begin{array}{l}<1.0 \\
48.8 \\
28.5 \\
21.95\end{array}$ & 26.6 & 5.48 & $\begin{array}{l}16.73- \\
58.9\end{array}$ \\
\hline $\begin{array}{l}\text { Waist Circumference: Males } \\
\text { Over } 40 \text { inches }\end{array}$ & 15.0 & 34.61 & 4.76 & $28-55$ \\
\hline $\begin{array}{l}\text { Waist Circumference: Females } \\
\text { Over } 35 \text { inches }\end{array}$ & 31.0 & 33.79 & 5.22 & $23-42$ \\
\hline $\begin{array}{l}\text { Lipids } \\
\text { High Density Lipids } \\
\text { Triglycerides } \\
\end{array}$ & & $\begin{array}{l}54.82 \\
106.85 \\
\end{array}$ & $\begin{array}{l}15.27 \\
50.95\end{array}$ & $\begin{array}{l}19-119 \\
35-422 \\
\end{array}$ \\
\hline $\begin{array}{l}\text { Blood Pressure } \\
\text { Systolic } \\
\text { Diastolic } \\
\end{array}$ & & $\begin{array}{l}114 \\
75 \\
\end{array}$ & $\begin{array}{l}14.0 \\
9.0 \\
\end{array}$ & $\begin{array}{l}80-148 \\
54-96 \\
\end{array}$ \\
\hline
\end{tabular}

$\%=$ percentage; $\mathrm{M}=$ mean of sample; $\mathrm{SD}=$ standard deviation of sample. $N=133$.

The remaining variables within the metabolic profile included body size (BMI, waist circumference), dyslipidemia (HDL, triglycerides), and blood pressure. Body mass index was calculated using the height and weight measured by the HC nurse during the biometric health exam. Height was measured using a stadiometer. The participants stood against the stadiometer without shoes. Height was measured by visual inspection of the stadiometer and recorded in feet and inches. Weight was measured using an electronic scale. Participants stood on the scale without shoes after emptying their pockets. Participants were not asked to change clothes. Body mass index was calculated using the standard formula: $\mathrm{BMI}=$ [pounds $/$ inches $]^{2} \mathrm{X}$ 703. The mean BMI was within overweight range $(M=26.6, \mathrm{SD}=5.48$, range $16.73-58.9)$. Only one participant was underweight $(\mathrm{BMI}=16.73)$. Approximately one-half the participants were within the normal range $(48.8 \%)$. One-half $(50.45 \%)$ were either in the overweight $(28.5 \%)$ or obese $(21.95 \%)$ range. 
The HC nurse measured waist circumference during the biometric health exam using a tape measure placed two inches above the umbilicus. The participants were not asked to remove or raise their clothing; the tape measure was placed over the clothing. The waist measurement was taken in inches. Waist measurements ranged from 23-55 inches for all participants, male and female $(M=33.79, S D=5.22)$. Higher than recommended waist measurements ( $\geq 35$ inches for females and $\geq 40$ inches for males) can indicate risk for metabolic disease, including prediabetes. Waist measurements for female participants $(n=46)$ ranged from $23-42$ inches $(M=32.93, \mathrm{SD}=6.02)$. Thirteen female participants $(31 \%)$ had waist measurements equal to or greater than 35 inches. Waist measurements for male participants $(n=83)$ ranged from $28-55$ inches $(M=34.61$, $S D=4.76)$. Nearly $15 \%$ of males had waist measurements equal to or greater than 40 inches.

Dyslipidemia was determined through two measures, high-density lipids (HDL) and triglycerides. The NI HC nurse obtained a blood sample for HDL and triglycerides during the biometric health exam. Nine participant EMR had missing lipid profile data. Participants $(n=124)$ were fasting for the exam. Low HDL levels $(\leq 39 \mathrm{mg} / \mathrm{dL})$ or high triglyceride levels $(>150 \mathrm{mg} / \mathrm{dL})$ can indicate risk for metabolic disease, including prediabetes. Participant HDL results ranged from $19-119 \mathrm{mg} / \mathrm{dL}(M=54.82, S D=15.27)$. Most participants $(87.3 \%$ ) had HDL levels at recommended levels (HDL $\geq 40 \mathrm{mg} / \mathrm{dL}$ ), and, therefore, were not at risk for prediabetes. Triglyceride results for the participants ranged from $35-422 \mathrm{mg} / \mathrm{dL}(M=106.85, \mathrm{SD}=59.65)$. Most of the 124 participants $(81.5 \%)$ had triglyceride levels that were at or below recommended levels $(\leq 150 \mathrm{mg} / \mathrm{dL})$, indicating that they were not at risk for prediabetes.

The HC nurse measured blood pressure during the biometric exam. Six participant EMR were missing blood pressure data $(n=127)$. The participants were asked to be in a 
sitting position with their feet flat on the floor. A standard sphygmomanometer with appropriate cuff size was used to take blood pressure. Blood pressure measurements were repeated only when the results were outside normal parameters. The second blood pressure, if indicated, was taken approximately 10 minutes later. Risk for metabolic disease, including prediabetes, is increased when either systolic blood pressure (SBP) or diastolic blood pressure (DBP) is elevated. Systolic blood pressure is considered high when readings are equal to or above $120 \mathrm{~mm} / \mathrm{Hg}$. Diastolic blood pressure is considered high when readings are equal to or above $80 \mathrm{~mm} / \mathrm{Hg}$. Participant SBP measurements ranged from $80-148 \mathrm{~mm} / \mathrm{Hg}(M=114, S D=14)$. Diastolic blood pressure measurements ranged from $54-96 \mathrm{~mm} / \mathrm{Hg}(M=75, S D=9)$. Approximately $63 \%$ of the 127 participants had normal blood pressure $(\mathrm{SBP}=62.9 \%, \mathrm{DBP}=63.9 \%)$, indicating low or no risk for prediabetes. High blood pressure was present in about one-third of the participants (SBP $=37 \%, \mathrm{DBP}=36.2 \%)$.

\section{Sociocultural Variables}

Sociocultural variables included in this study were: age, sex, race/ethnicity, education, family history, job role, job hours, stress, job strain, and job imbalance. A summary of demographic and job related variables (age, sex, race/ethnicity, education, job role, job hours) can be found in Table 4. Note that none of the participants reported family history of T2DM. A summary of the remaining sociocultural variables (stress, job strain, job imbalance) can be found in Table 6. Demographic variables (age, sex, race/ethnicity, education, family history, job role, job hours) were previously described. 
Table 6. Summary of Sociocultural Variables.

\begin{tabular}{l|lll}
\hline & M & SD & Range \\
\hline Perceived Stress Scale & 23.89 & 6.44 & $10-40$ \\
& & & \\
\hline Job Strain & 1.28 & 0.25 & $0.86-2.46$ \\
& & & \\
\hline Job Imbalance & 11.91 & 2.02 & $5-17$ \\
\hline
\end{tabular}

$\mathrm{M}=$ mean of sample; $\mathrm{SD}=$ standard deviation of sample.

Three sociocultural variables measured stress: global and event-specific stress and two types of work stress: job strain and job imbalance. The Perceived Stress Scale (PSS) included in the online participant survey measured global and event-specific stress. Higher scores indicate greater stress. Participant $(N=133)$ scores ranged from 10-40, which spans the range of possible scores. The mean PSS score was $23.89(S D=6.44)$, indicating a moderate degree of global and event-related stress.

The Job Content Questionnaire (JCQ) and the Effort-Reward-Imbalance (ERI) scales measured work stress. Both scales were part of the online participant surveys. Each scale measured one type of work stress. The JCQ scale measured job strain, the interaction effect of physical plus psychological job demands and control over workrelated decisions. The job ratio term is calculated by multiplying the job demands score by two then dividing the total by the decisional latitude score for each participant. A ratio term greater than one is used to indicate job strain. This ratio term allows comparison to national standards and allow studies in a "small population or single plant" to compare their findings to national averages on all scales (Karasek, 1986, p.1). A summary of job strain can be found in Table 7. Seven participants had incomplete JCQ surveys. Of the participants who had complete JCQ surveys $(n=126)$, job strain was defined and calculated as recommended. 
Job strain was calculated using the ratio interaction formula recommended with the Job Content Questionnaire and User's Guide (Karasek, 1986), where job demands is multiplied by two then divided by decisional latitude. Using the job strain ratio formula, an individual score greater than one measures job strain. The job strain calculations indicated that most of the participants had job strain scores greater than one $(92.1 \%)$. Participants' scores ranged from 0.86 to 2.46 . The mean job strain score was $1.28(S D=$ 0.25). Computation of a $z$-score can be used to "obtain a sense of magnitude" of the components of job strain, job demands and decisional latitude (Karasek, 1986, p. 15). The $z$-score for job demands was -0.13 . The $z$-score for decisional latitude was -1.44 . Based on $z$-scores, participants reported low job demands and low decisional latitude.

Table 7. Job strain.

\begin{tabular}{l|lllll}
\hline \multirow{2}{*}{$\begin{array}{l}\text { Job Strain Ratio: Calculated Job } \\
\text { Strain }\end{array}$} & $\%$ & $\mathrm{M}$ & Median & SD & Range \\
\cline { 2 - 5 } & & 1.28 & & 0.25 & $0.86-2.46$ \\
$\quad<1$ (yes) & 92.1 & & & & \\
$\quad$ & 7.9 & & & & \\
\hline
\end{tabular}

$\%=$ percent $; \mathrm{M}=$ mean of sample $; \mathrm{SD}=$ standard deviation of sample.

The ERI scale measured job imbalance, the interaction effect of the perceived amount of effort at work and reward received in return. Job imbalance is calculated by dividing effort scores by reward scores and multiplying by a correction term. A summary of job imbalance was described in Table 6. The effort scale measured the amount of extrinsic effort. Scores on the effort scale potentially ranged from 5-25 (median $=15$ ). Higher scores on the effort scale indicated greater levels of extrinsic effort. Participant scores on the effort scale ranged from 5-17 $(M=11.91, S D=2.02$, median $=12)$. The participant extrinsic effort median score was lower than the scale median, indicating lower perceived extrinsic effort in this sample (12 vs. 21 , respectively). The reward scale 
measured the amount of perceived reward received at work. Range of scores for the reward scale potentially were 11-55 (median $=33$ ). Lower scores on the reward scale indicate fewer occupational rewards received by the worker. Ten participants had incomplete ERI surveys $(n=123)$. Participant scores on the reward scale ranged from 12$40(M=30.84, S D=4.79$, median $=31)$. The participant perceived reward median score was lower than the survey median, indicating lower perceived occupational rewards (31 vs. 33, respectively). High job imbalance was confirmed when scores were greater than one. Low job imbalance was confirmed when scores were less than one. Scores for the ERI ranged from 0.44 to $1.87(M=1.07, S D=0.21)$. Only one participant has perfect job balance (score $=1$ ). Approximately $64 \%$ of the participants reported high job imbalance. Approximately $34 \%$ of the participants reported low job imbalance.

\section{Health Behavior Variables}

Health behavior variables included in this study were: diet, exercise, sleep, smoking, alcohol, and coping. A summary of the health behavior variables can be found in Table 8. Each variable was measured with survey questions included as part of the online participant surveys. 
Table 8. Summary of Health Behavior Variables.

\begin{tabular}{l|llll}
\hline \multirow{2}{*}{ Diet } & $\%$ & M & SD & Range \\
\cline { 2 - 5 } Mostly Healthy & & & & \\
Healthy and Unhealthy & 36.1 & & & \\
Mostly Unhealthy & 11.0 & & & \\
\hline Exercise & 42.6 & & & \\
$\quad$ Sometimes or Often (self-report) & 75.4 & & & \\
$\quad$ Meeting USHHS guidelines & 66 & & & \\
\hline Alcohol: Occasional & 75.9 & & & \\
\hline Smoking: Non-smoker & 72.0 & & & \\
\hline Sleep & & 6.94 & 28.9 & \\
$\quad$ No insomnia & 58.5 & & & \\
Sub-Threshold Insomnia & 29.0 & & & \\
Moderate Insomnia & 10.0 & & & \\
Severe Insomnia & 3.0 & & & \\
\hline Stress & & 23.89 & 6.44 & \\
\hline Coping & & 37.37 & 3.46 & $29-29$ \\
\hline
\end{tabular}

$\%=$ percent $; \mathrm{M}=$ mean of sample $; \mathrm{SD}=$ standard deviation of sample.

The diet variable was measured through the Rapid Eating and Activity Assessment for Patients (REAP-S) scale, which measures compliance with USDA dietary guidelines. Responses on the REAP-S included healthy and unhealthy eating behaviors in 127 participants (16 surveys were incomplete). No participant was fully compliant with USDA guidelines. A positive score on the REAP-S indicated that participants tended to have more healthy-eating behaviors than unhealthy-eating behaviors, which suggested that participants met USDA guidelines more often than not. A negative score indicated that participants tended to have more unhealthy-eating behaviors and failed to meet USDA dietary guidelines more often than not. A score of zero indicated a perfect balance between healthy and unhealthy eating behaviors. Scores on the REAP-S ranged from -12 to 12 , which indicated a broad range of compliance to USDA dietary guidelines $(M=-$ $0.71, S D=5.51)$. Approximately one-third $(36.1 \%)$ of the participants met USDA dietary guidelines by reporting mostly eating a healthy diet. Nearly one-half (42.6\%) reported 
consistently eating an unhealthy diet. Eleven percent of the participants reported a diet that evenly represented healthy and unhealthy eating habits.

The Godin Leisure Time Exercise Questionnaire (GLTEQ) included in the online participant surveys measured the exercise variable. The USHHS recommends that adults exercise 30 minutes per day most of the days of the week (150 minutes per week). The GLTEQ measured compliance with the USHHS exercise recommendations. Questions on the GLTEQ asked about types of exercise, frequency, and exertion. Scores for the GLTEQ were calculated by multiplying the frequency of exercise with the type of exercise. Twelve surveys were incomplete $(n=121)$. Most of the participants reported exercising sometimes or often $(75.37 \%)$, as recommended by the USHHS. Scores equal to or higher than 30 indicate meeting USHHS exercise guidelines. Sixty-six percent of participants scored 30 or higher $(M=45.02, S D=28.9)$.

The sleep variable was measured by the Insomnia Severity Index (ISI), which measured difficulty with sleep by assessing the severity of recent nighttime and daytime insomnia. The ISI was included with the online participant surveys. The scores for ISI sort insomnia by severity. Scores less than eight indicated no insomnia. Scores ranging from 8-14 indicated sub-threshold insomnia. Scores ranging from 15-21 indicated moderate to severe insomnia, and scores ranging from 22-28 indicated severe insomnia. Only one survey was incomplete. The participant scores $(n=132)$ ranged from $0-23(M=$ $6.94, S D=28.90)$. Most of the participants $(58.5 \%)$ had scores that showed no significant clinical insomnia. Nearly $29 \%$ of participants responded in the sub-threshold insomnia range, and approximately $10 \%$ of the participants scored in the moderate to severe clinical insomnia range. Less than $3 \%$ of the participants scored in the severe clinical insomnia category. 
The smoking variable measured the use of tobacco cigarettes. The participant was asked to answer "yes" or "no" to the question, "Do you currently smoke?" as part of the demographic questions included in the online participant surveys. The recommendation from the USHHS states that any amount of smoking is harmful to health. Participants scored a "yes" if they indicated current smoking. Most of the participants did not smoke $(72.1 \%, N=133)$.

A set of three questions included in the demographic section of the online participant surveys measured the alcohol variable. The questions asked participants to respond "yes" or "no" to alcohol intake. If the answer was "yes", two follow-up questions asked the frequency of alcohol intake and the amount per instance. Light to moderate alcohol intake has been positively associated with health. No alcohol or heavy alcohol is negatively associated with health. Most $(75.9 \%, \mathrm{~N}=133)$ of the participants responded "yes" to drinking alcoholic beverages. Participants indicated that frequency of alcohol intake was light to moderate, either 1-2 times per month $(30.84 \%)$ or 1-2 times per week (26.7\%). Nearly $4 \%$ of the participants drank alcohol daily. Of the participants who drank alcoholic beverages, most (70.9\%) drank 1-2 drinks per instance.

The Brief Approach/Avoidance Coping Questionnaire (BACQ) included with the online participant surveys assessed the coping variable. The BACQ survey measured approach/avoidance coping strategies. Higher scores indicated use of approach-oriented coping strategies. Possible scores range from 5-100. Three surveys were incomplete ( $n=$ 130). Participant scores ranged from 29-49. The mean score was $37.37(S D=3.46)$, indicating avoidant-type coping. 


\section{Findings fRom DAta ANALYSES by RESEARCh QUESTION}

\section{Prevalence of Prediabetes}

It is estimated that $35 \%$ of US adults aged 20 years and older have prediabetes, even after adjusting for age and gender differences (CDC, 2011). Prediabetes is confirmed when fasting blood glucose results are within 100-126mg/dL during laboratory testing. In this study, prediabetes was measured through a blood sample collection for fasting blood glucose during the biometric screening exam. The instances of prediabetes within the participant sample were much lower than what is reported in the US adult population. Out of the 126 participants with fasting blood glucose results, only 24 (19\%) had prediabetes. The results of this study showed a lower prevalence of prediabetes in this sample compared to the US population. The prevalence of prediabetes was $19 \%$ in this sample of full-time employees. A summary of prediabetes can be found in Table 8 . Participants with prediabetes were mostly middle-aged White $(70.8 \%)$ males $(66.6 \%)$. More than one-half had BMI scores above the normal range. The majority $(62.5 \%)$ of females had waist circumference measurements as recommended ( $<35$ inches). Most (75\%) males had waist circumference measurements as recommended ( $<40$ inches). The majority of participants with prediabetes has HDL (67.7\%), triglyceride (87.5\%), systolic blood pressure $(62.5 \%)$, and diastolic blood pressure $(67.7 \%)$ results within normal ranges. Very few participants $(0.8 \%)$ were in compliance with dietary and exercise guidelines. Seventy-four percent of participants with prediabetes reported drinking 16 or

more ounces of sugared drinks at least three times per week. Approximately one-third (38\%) reported drinking moderate alcohol (as recommended). Most (83\%) were nonsmokers. None of the participants with prediabetes had severe insomnia. 
Table 9. Summary of Prediabetes.

Age $43.5 \pm 12.52(25-63) . n=126 . \%=$ percent.

\begin{tabular}{|c|c|}
\hline & $\%$ \\
\hline $\begin{array}{l}\text { Prediabetes }(\mathrm{FBG} \geq 100 \mathrm{mg} / \mathrm{dL}) \\
\text { Yes } \\
\text { No }\end{array}$ & $\begin{array}{l}19.1 \\
80.9\end{array}$ \\
\hline $\begin{array}{l}\text { Sex } \\
\text { Male } \\
\text { Female }\end{array}$ & $\begin{array}{l}66.6 \\
33.3\end{array}$ \\
\hline $\begin{array}{l}\text { Race/Ethnicity in Prediabetes } \\
\text { White } \\
\text { African American } \\
\text { Asian } \\
\text { Native American } \\
\text { Hispanic }\end{array}$ & $\begin{array}{l}70.8 \\
0 \\
20.8 \\
4.2 \\
8.3\end{array}$ \\
\hline $\begin{array}{l}\text { BMI } \\
\text { Underweight } \\
\text { Normal Range } \\
\text { Overweight } \\
\text { Obese }\end{array}$ & $\begin{array}{l}0.41 \\
29.2 \\
33.3 \\
33.3\end{array}$ \\
\hline $\begin{array}{l}\text { Waist Circumference } \\
\text { Females } \\
\quad<35 \text { inches } \\
>35 \text { inches } \\
\text { Males } \\
\quad<40 \text { inches } \\
>40 \text { inches }\end{array}$ & $\begin{array}{l}62.5 \\
37.5 \\
75.0 \\
25.0\end{array}$ \\
\hline $\begin{array}{l}\text { HDL } \\
>40 \mathrm{mg} / \mathrm{dL} \\
<40 \mathrm{mg} / \mathrm{dL}\end{array}$ & $\begin{array}{r}66.7 \\
33.3\end{array}$ \\
\hline $\begin{array}{l}\text { Triglycerides } \\
<199 \mathrm{mg} / \mathrm{dL} \\
>199 \mathrm{mg} / \mathrm{dL} \\
\end{array}$ & $\begin{array}{l}87.5 \\
12.5\end{array}$ \\
\hline $\begin{array}{l}\text { Systolic Blood Pressure } \\
\quad<120 \mathrm{mmHg} \\
>120 \mathrm{mmHg} \\
\text { Diastolic Blood Pressure } \\
\quad<80 \mathrm{mmHg} \\
>80 \mathrm{mmHg}\end{array}$ & $\begin{array}{l}62.5 \\
37.5 \\
66.7 \\
33.3\end{array}$ \\
\hline $\begin{array}{l}\text { Diet } \\
\text { Met } \\
\text { Not Met }\end{array}$ & $\begin{array}{l}0.8 \% \\
99.2 \%\end{array}$ \\
\hline $\begin{array}{l}\text { Exercise } \\
\text { Met } \\
\text { Not Met }\end{array}$ & $\begin{array}{l}0.8 \% \\
99.2 \%\end{array}$ \\
\hline $\begin{array}{l}\text { Alcohol: occasional } \\
\text { Smoking: non-smoker }\end{array}$ & $\begin{array}{l}38 \% \\
83 \%\end{array}$ \\
\hline $\begin{array}{l}\text { Sleep } \\
\text { No Insomnia } \\
\text { Sub-Threshold Insomnia } \\
\text { Moderate Insomnia }\end{array}$ & $\begin{array}{l}41.7 \\
33.3 \\
20.8\end{array}$ \\
\hline
\end{tabular}




\section{Prevalence of Job Strain}

It is estimated that $80 \%$ of US workers report stress at work, with $40 \%$ of US workers reporting work stress to be extremely stressful (NIOSH, 1999). The Job Content Questionnaire was used to determine job strain. The calculation of job strain in the participant sample through the job strain ratio term was higher than estimates of work stress in US workers. A summary of job strain is presented in Table 10. Out of 126 participants who reported data on this variable, only 10 did not have job strain as calculated by the Job Content Questionnaire's job strain ratio term. Nearly all of the participants $(92.2 \%)$ reported job strain using the ratio term calculation. The prevalence rate for job strain was $92 \%$ in this sample of full-time employees.

Table 10. Summary of Job Strain.

\begin{tabular}{l|llll}
\hline \multirow{3}{*}{ Job Strain } & $\%$ & M & SD & Range \\
\cline { 2 - 5 } Yes & & & & \\
No & 92.1 & & & \\
\hline Age & 7.9 & & & \\
\hline Sex & & 37.46 & 9.93 & $25-63$ \\
Male & & & & \\
Female & 64.8 & & & \\
\hline Race/Ethnicity & 35.3 & & \\
White & & & & \\
African American & 81.0 & & & \\
Asian & 2.5 & & & \\
Native American & 17.2 & & & \\
Hispanic & 2.5 & & & \\
\hline
\end{tabular}

$\%=$ percent $; \mathrm{M}=$ mean; $\mathrm{SD}=$ standard deviation $(n=126)$.

\section{Prevalence of Job Imbalance}

It is estimated that $80 \%$ of US workers experience stress at work, and $40 \%$ report work to be extremely stressful (NIOSH, 1999). Report of work stress in the participant sample as measured by job imbalance was lower than estimates of overall work stress reported by US workers. The Effort-Reward-Imbalance questionnaire was used to 
determine job imbalance. A summary of job imbalance is in Table 11 . Out of the 123 participants who reported data on this variable, approximately one-third (35.8\%) did not have job imbalance. The prevalence rate for job imbalance was $64 \%$ in this sample of full-time employees.

Table 11. Summary of Job Imbalance.

\begin{tabular}{l|llll}
\hline \multirow{3}{*}{$\begin{array}{l}\text { Job Imbalance } \\
\text { High }\end{array}$} & $\%$ & M & SD & Range \\
\cline { 2 - 4 } Low & & & & \\
\hline Age & 64.2 & & & \\
& 34.1 & & & \\
\hline Sex & & 37.07 & 9.84 & $25-63$ \\
Male & & & & \\
Female & 64.6 & & & \\
\hline Race/Ethnicity & 35.4 & & \\
White & & & & \\
African American & 86.1 & & \\
Asian & 3.7 & & & \\
Native American & 10.1 & & & \\
Hispanic & 3.7 & & & \\
\hline
\end{tabular}

$\%=$ percent $; \mathrm{M}=$ mean; $\mathrm{SD}=$ standard deviation $(n=126)$.

\section{Relationship Between Job Strain and Job Imbalance}

As stated previously, many of the participants reported job strain and job imbalance. Histogram and z-score analyses showed that both job strain and job imbalance scores were not normally distributed. A one-tailed Spearman's rho correlation coefficient (Q) analysis was used to determine the relationship between job strain and job imbalance. Alpha level was at the 0.05 level. The Spearman's rho correlation analysis was selected in order to account for the skewed distribution of both variables and the unequal sample size for job strain and job imbalance scores ( $n=126$ vs. $n=123$, respectively). Results of the analysis showed that there was statistically significant positive correlation. A summary of job imbalance is in Table 12. Results of the analysis showed a statistically 
significant association between job strain and job imbalance in the participant sample of full time employees of the computer company, $\varrho(115)=0.346, p<0.01$.

Table 12. Summary of Spearman's rho correlation for job strain and job imbalance.

\begin{tabular}{|c|c|c|c|c|}
\hline & & & $\begin{array}{c}\text { Corrected } \\
\text { ERI }\end{array}$ & JCQ_correct \\
\hline \multirow[t]{6}{*}{ Spearman's rho } & \multirow[t]{3}{*}{ Corrected ERI } & $\begin{array}{l}\text { Correlation } \\
\text { Coefficient }\end{array}$ & 1.000 & $.346^{* *}$ \\
\hline & & Sig. (1-tailed) & . & .000 \\
\hline & & $\mathrm{N}$ & 123 & 117 \\
\hline & \multirow[t]{3}{*}{ JCQ_correct } & $\begin{array}{l}\text { Correlation } \\
\text { Coefficient }\end{array}$ & $.346^{* *}$ & 1.000 \\
\hline & & Sig. (1-tailed) & .000 & . \\
\hline & & $\mathrm{N}$ & 117 & 126 \\
\hline
\end{tabular}

JCQ_correct = Job Stain; Corrected ERI = Job Imbalance.

\section{Relationship Between Job Strain and Prediabetes}

Of the 126 participants who had complete survey data on the JCQ, nearly all had job strain $(92.2 \%, n=116)$, and most of the participants did not have prediabetes $(80.9 \%$, $n=102$ ). Prevalence of job strain was $92 \%$, while prevalence of prediabetes was $19 \%$. Job strain scores results were not normally distributed, and, therefore, did not meet the criteria for Pearson's product-moment correlation. A one-tailed Spearman's rho correlation coefficient (Q) analysis was used to determine the relationship between job strain and fasting blood glucose. Alpha level was set at 0.05. The Spearman's rho correlation analysis was selected to account for the skewed job strain scores. A summary of job imbalance is in Table 13. Results of the analysis showed that job strain and prediabetes were not significantly correlated in the participant sample of full time employees of the computer company, $\mathrm{Q}(111)=-0.94, p=0.16$. 
Table 13. Summary of Spearman's rho correlation for job strain and prediabetes.

\begin{tabular}{|lll|r|r|}
\hline & & JCQ_correct & \multicolumn{1}{c|}{ FBS } \\
\hline Spearman's rho & JCQ_correct & Correlation & 1.000 & -.094 \\
& & Coefficient &. & .160 \\
& & Sig. (1-tailed) & 126 & 113 \\
\cline { 2 - 4 } & $\mathrm{N}$ & -.094 & 1.000 \\
\cline { 2 - 5 } & FBS Correlation & .160 &. \\
& & Coefficient & 113 & 126 \\
\hline & Sig. (1-tailed) & & \\
& $\mathrm{N}$ & & \\
& & &
\end{tabular}

JCQ_correct $=$ Job Strain; FBS = Prediabetes.

\section{Relationship Between Job Imbalance and Prediabetes}

Of the 123 participants who had complete survey data on the ERI, almost two thirds $(64 \%, n=79)$ reported high job imbalance. Most of the participants did not have prediabetes $(80.9 \%, n=99)$. Scores for job imbalance results were not normally distributed, and, therefore, did not meet the criteria for Pearson's product-moment correlation. A one-tailed Spearman's rho correlation coefficient (Q) analysis was used to determine the relationship between job imbalance and prediabetes. Alpha level was set at 0.05. A summary of job imbalance is in Table 14. Results of the analysis showed that there was a statistically significant negative correlation between job imbalance and prediabetes this sample, $(\varrho(109)=-0.192, p=0.022)$. 
Table 14. Summary of Spearman's rho correlation for job imbalance and prediabetes.

\begin{tabular}{|c|c|c|c|c|}
\hline & & & FBS & $\begin{array}{c}\text { Corrected } \\
\text { ERI }\end{array}$ \\
\hline \multirow[t]{6}{*}{ Spearman's rho } & \multirow[t]{3}{*}{ FBS } & $\begin{array}{l}\text { Correlation } \\
\text { Coefficient }\end{array}$ & 1.000 & $-.192^{*}$ \\
\hline & & Sig. (1-tailed) & . & .022 \\
\hline & & $\mathrm{N}$ & 126 & 110 \\
\hline & \multirow[t]{3}{*}{ Corrected ERI } & $\begin{array}{l}\text { Correlation } \\
\text { Coefficient }\end{array}$ & $-.192^{*}$ & 1.000 \\
\hline & & Sig. (1-tailed) & .022 & . \\
\hline & & $\mathrm{N}$ & 110 & 123 \\
\hline
\end{tabular}

Corrected ERI = Job Imbalance; FBS $=$ Prediabetes.

\section{Relationship Between Job Strain and Stress}

The Job Content Questionnaire (JCQ) measured job strain. Most of the 126 participants reported job strain as measured by the JCQ job strain ratio term (92.1). The Perceived Stress Scale (PSS) measured global and event-specific stress. Participant scores $(N=133)$ on the PSS encompassed all possible scores and ranged from 10-40. The participants' mean scores showed a moderate degree of global and event-specific stress $(M=23.89, S D=6.44)$. Scores from the JCQ were not normally distributed, and therefore, did not meet the criteria for Pearson's product-moment correlation. A onetailed Spearman's rho correlation coefficient (o) analysis was used to determine the relationship between job strain and global and event-specific stress. Spearman's rho correlation analysis is preferred when variables are not normally distributed. Alpha level was set at 0.05. A summary of job imbalance is in Table 15. Results of the analysis showed that there was a statistically significant positive correlation between job strain and global and event-specific stress in this sample of full time employees of the computer company, $\mathrm{Q}(121)=0.337, p<0.001$ in this sample. 
Table 15. Summary of Spearman's rho correlation for job strain and global and eventspecific stress.

\begin{tabular}{|c|c|c|c|c|}
\hline & & & PSS & JCQ_correct \\
\hline \multirow[t]{6}{*}{ Spearman's rho } & \multirow[t]{3}{*}{ PSS } & $\begin{array}{l}\text { Correlation } \\
\text { Coefficient }\end{array}$ & 1.000 & $.337^{k * \hbar}$ \\
\hline & & Sig. (1-tailed) & . & .000 \\
\hline & & $\mathrm{N}$ & 136 & 123 \\
\hline & \multirow[t]{3}{*}{ JCQ_correct } & $\begin{array}{l}\text { Correlation } \\
\text { Coefficient }\end{array}$ & $.337^{* *}$ & 1.000 \\
\hline & & Sig. (1-tailed) & .000 & \\
\hline & & $\mathrm{N}$ & 123 & 126 \\
\hline
\end{tabular}

JCQ_correct $=$ Job Strain; PSS = Global and Event-Specific Stress.

\section{Relationship Between Job Imbalance and Stress}

The Effort-Reward-Imbalance Scale measured job imbalance. Approximately $64 \%$ of the participants with complete surveys $(n=123)$ reported job imbalance. Participant scores $(N=133)$ on the PSS ranged from 10-40, which covered the range of all possible scores. The participants' mean scores showed a moderate degree of global and event-specific stress $(M=23.89, S D=6.44)$. The job imbalance results were not normally distributed, and therefore, did not meet the criteria for Pearson's productmoment correlation. A Spearman's rho correlation coefficient (@) analysis was used to determine the relationship between job imbalance and global and event-specific stress. The Spearman's rho correlation analysis is preferred when variables are not normally distributed. Alpha level was set at 0.05. A summary of job imbalance is in Table 16 . Results of the analysis showed that there was statistically significant positive correlation between job strain and global and event specific in this sample of full time employees of the computer company, $\varrho(119)=0.261, p=0.002$. 
Table 16. Summary of Spearman's rho correlation for job imbalance and global and event-specific stress.

\begin{tabular}{|c|c|c|c|c|}
\hline & & & $\begin{array}{c}\text { Corrected } \\
\text { ERI }\end{array}$ & PSS \\
\hline \multirow[t]{6}{*}{ Spearman's rho } & \multirow[t]{3}{*}{ Corrected ERI } & $\begin{array}{l}\text { Correlation } \\
\text { Coefficient }\end{array}$ & 1.000 & $.261^{* *}$ \\
\hline & & Sig. (1-tailed) & . & .002 \\
\hline & & $\mathrm{N}$ & 123 & 121 \\
\hline & \multirow[t]{3}{*}{ PSS } & $\begin{array}{l}\text { Correlation } \\
\text { Coefficient }\end{array}$ & $.261^{* * *}$ & 1.000 \\
\hline & & Sig. (1-tailed) & .002 & . \\
\hline & & $\mathrm{N}$ & 121 & 136 \\
\hline
\end{tabular}

Corrected ERI = Job Imbalance; PSS = Global and Event-Specific Stress.

\section{Predictors of Prediabetes}

Before calculating the regression analysis, bivariate correlations were computed to identify which variables could be used as predictor variables. A one-tailed Spearman's rho (Q) correlation coefficient analysis was selected for the bivariate correlation analyses in order to account for skewness and unequal sample sizes. Alpha level was set at 0.05 . The Spearman's rho correlation analysis results indicated that six independent variables were statistically significantly associated with prediabetes. A summary of bivariate correlations can be found on Table 16. These variables were: participant age, BMI, waist circumference, exercise scores, and job imbalance, all of which were continuous variables. There was a statistically significant positive correlation between age and prediabetes in this sample, $\mathrm{Q}(131)=0.196, p=0.016$. There was a statistically significant negative correlation between body mass index and prediabetes in this sample, $\varrho(124)=-$ $0.192, p=0.017$. Waist circumference was statistically significantly positively correlated with prediabetes in this sample, $\mathrm{Q}(122)=0.222, p=0.007$. There was a statistically significant positive correlation between exercise and prediabetes in this sample, $\varrho(107)=$ 
$-2.06, p=0.016$. There was a statistically significant negative correlation between job imbalance and prediabetes in this sample, $\mathrm{Q}(121)=-0.192, p=0.022$. Use of alcohol was scored as a dichotomous variable (yes $=1$ or no $=0$ ). Fasting blood sugar results were used to create a dichotomous prediabetes variable using recommended cut scores (yes if $>99 \mathrm{mg} / \mathrm{dL}$ or no if $<100 \mathrm{mg} / \mathrm{dL}$ ). There was a statistically significant negative correlation between alcohol and prediabetes in this sample, $\varrho(122)=-0.198, p=0.014$. 
Table 17. Spearman's rho bivariate correlations.

\begin{tabular}{|c|c|c|c|c|c|c|c|c|c|c|c|c|c|c|c|c|}
\hline & & Age & Smoking & Alcoho & Diet & Exercisg & ISI & BMI & Waist & SBP & DBP & HDL & Trig & FBG & $\mathrm{JCQ}$ & ERI \\
\hline \multirow[t]{3}{*}{ Age } & $\mathrm{Oq}$ & 1.000 & & & & & & & & & & & & & & \\
\hline & Sid & & & & & & & & & & & & & & & \\
\hline & $\mathrm{N}$ & 133 & & & & & & & & & & & & & & \\
\hline \multirow[t]{3}{*}{ Smoking } & $\mathrm{OC}$ & .021 & 1.000 & & & & & & & & & & & & & \\
\hline & Sid্ব & .408 & . & & & & & & & & & & & & & \\
\hline & $\mathrm{N}$ & 131 & 139 & & & & & & & & & & & & & \\
\hline \multirow[t]{3}{*}{ Alcohol } & $\mathrm{Oq}$ & $-.206^{\circ}$ & $.167^{+}$ & 1.000 & & & & & & & & & & & & \\
\hline & Sid & .009 & .025 & - & & & & & & & & & & & & \\
\hline & $\mathrm{N}$ & 131 & 139 & 139 & & & & & & & & & & & & \\
\hline \multirow[t]{3}{*}{$\overline{\text { Diet }}$} & $\mathrm{CO}$ & .015 & .001 & .053 & 1.000 & & & & & & & & & & & \\
\hline & Sif̧ & .435 & .497 & 278 & . & & & & & & & & & & & \\
\hline & $\bar{N}$ & 123 & 127 & 127 & 127 & & & & & & & & & & & \\
\hline \multirow[t]{3}{*}{ Exercise } & $\mathrm{CO}$ & $=.027$ & $=.067$ & .073 & .018 & 1.000 & & & & & & & & & & \\
\hline & Sif̧ & .387 & .234 & 212 & .427 & - & & & & & & & & & & \\
\hline & $\mathrm{N}$ & 113 & 121 & 121 & 114 & 121 & & & & & & & & & & \\
\hline \multirow[t]{3}{*}{$\overline{\text { ISI }}$} & $\mathrm{Oq}$ & .054 & .029 & .023 & $.231^{\circ}$ & .063 & 1.000 & & & & & & & & & \\
\hline & Siç & .273 & .369 & 397 & .005 & .247 & . & & & & & & & & & \\
\hline & $\mathrm{N}$ & 125 & 132 & 132 & 126 & 119 & 132 & & & & & & & & & \\
\hline \multirow[t]{3}{*}{$\overline{\text { BMI }}$} & $\mathrm{OC}$ & $.181^{2}$ & .018 & .117 & $.268^{2}$ & $-.229^{17}$ & .029 & \begin{tabular}{|l|}
1.000 \\
\end{tabular} & & & & & & & & \\
\hline & Sifa & .025 & .423 & .101 & .002 & .009 & .377 & . & & & & & & & & \\
\hline & $\mathrm{N}$ & 118 & 121 & 121 & 112 & 106 & 116 & 123 & & & & & & & & \\
\hline \multirow[t]{3}{*}{ Waist } & $\mathrm{Cq}$ & .084 & .016 & .115 & $-324^{2}$ & $-.25 g^{n}$ & .005 & $790^{\circ}$ & 1.000 & & & & & & & \\
\hline & Sid্ব & .180 & .432 & .104 & .000 & .004 & .480 & .000 & . & & & & & & & \\
\hline & $\mathrm{N}$ & 120 & 123 & 123 & 114 & 107 & 118 & 123 & 125 & & & & & & & \\
\hline \multirow[t]{3}{*}{$\overline{\text { SBP }}$} & $\mathrm{CO}$ & $=.043$ & .018 & .099 & $.331^{\prime \prime}$ & .037 & -.061 & \begin{tabular}{|l|}
$473^{\prime \prime}$ \\
\end{tabular} & $.435^{\prime}$ & 1.000 & & & & & & \\
\hline & Siḑ & .322 & .423 & .136 & \begin{tabular}{|l|}
.000 \\
\end{tabular} & 351 & .253 & \begin{tabular}{|l|}
.000 \\
\end{tabular} & .000 & . & & & & & & \\
\hline & $\overline{\mathrm{N}}$ & 121 & 125 & 125 & 115 & 108 & 120 & 123 & 125 & 127 & & & & & & \\
\hline \multirow[t]{3}{*}{$\overline{\mathrm{DBP}}$} & $\mathrm{Cq}$ & $=.046$ & .110 & .090 & $-330^{\circ}$ & .005 & -.051 & $466^{\circ}$ & $.456^{\circ}$ & $.762^{\circ}$ & 1.000 & & & & & \\
\hline & Sif & .308 & .112 & .160 & .000 & 479 & .291 & .000 & .000 & .000 & . & & & & & \\
\hline & $\mathrm{N}$ & 121 & 125 & 125 & 115 & 108 & 120 & 123 & 125 & 127 & 127 & & & & & \\
\hline \multirow[t]{3}{*}{$\overline{\mathrm{HDL}}$} & $\mathrm{Cq}$ & $.221^{\circ}$ & .026 & .077 & \begin{tabular}{|l|}
$.198^{\circ}$ \\
\end{tabular} & .008 & .143 & \begin{tabular}{|l|l|}
.157 \\
\end{tabular} & $-371^{2}$ & $-.242^{\circ}$ & $-.239^{2}$ & 1.000 & & & & \\
\hline & Siḑ & .008 & .386 & .197 & .022 & 468 & .059 & \begin{tabular}{|l|}
.042 \\
.04
\end{tabular} & .000 & .003 & .004 & . & & & & \\
\hline & $\mathrm{N}$ & 120 & 124 & 124 & 115 & 108 & 120 & 122 & 124 & 126 & 126 & 126 & & & & \\
\hline \multirow[t]{3}{*}{ Trig } & $\mathrm{Od}$ & .143 & .077 & $-.151^{\prime}$ & \begin{tabular}{|c|}
$.161^{\prime}$ \\
\end{tabular} & $-.257^{m+1}$ & -117 & $424^{\circ}$ & $.387^{\circ}$ & \begin{tabular}{|l|}
.297 \\
\end{tabular} & $.318^{\circ}$ & -352 & 1.000 & & & \\
\hline & Sif̧ & .060 & .200 & .048 & \begin{tabular}{|l|}
.045 \\
\end{tabular} & .004 & .103 & \begin{tabular}{|l|}
.000 \\
\end{tabular} & .000 & .000 & .000 & .000 & . & & & \\
\hline & $\mathrm{N}$ & 119 & 122 & 122 & 113 & 106 & 118 & 120 & 122 & 124 & 124 & 124 & \begin{tabular}{|l|}
124 \\
\end{tabular} & & & \\
\hline \multirow[t]{3}{*}{$\overline{\mathrm{FBG}}$} & $\mathrm{Cq}$ & $.196^{\circ}$ & -.045 & $=.198$ & -145 & $-.206^{1}$ & .059 & \begin{tabular}{|l|}
192 \\
\end{tabular} & $.222^{\circ}$ & .056 & .124 & $\begin{array}{l}.147 \\
\end{array}$ & .077 & \begin{tabular}{|l|}
1.000 \\
\end{tabular} & & \\
\hline & Sif̧ & .016 & .311 & .014 & \begin{tabular}{|l|}
.061 \\
\end{tabular} & .016 & .262 & \begin{tabular}{|l|}
.017 \\
\end{tabular} & .007 & .267 & .084 & .051 & .199 & . & & \\
\hline & $\mathrm{N}$ & 120 & 124 & 124 & 115 & 108 & 120 & 122 & 124 & 126 & 126 & 126 & 124 & 126 & & \\
\hline \multirow[t]{3}{*}{$\overline{\mathrm{JCQ}}$} & $\mathrm{CO}$ & .117 & $.149^{2}$ & .131 & -0.060 & -.038 & $.186^{\circ}$ & \begin{tabular}{|l|}
-018 \\
\end{tabular} & \begin{tabular}{|l|}
-0.048 \\
\end{tabular} & $=.042$ & .000 & .148 & -.061 & \begin{tabular}{|l|}
-094 \\
\end{tabular} & 1.000 & \\
\hline & Sif̧ & .104 & .048 & .072 & \begin{tabular}{|l|}
.257 \\
\end{tabular} & 344 & .019 & \begin{tabular}{|l|}
.425 \\
\end{tabular} & .308 & .328 & .500 & .058 & .264 & \begin{tabular}{|l|}
.160 \\
\end{tabular} &. & \\
\hline & $\mathrm{N}$ & 118 & 126 & 126 & 120 & 113 & 124 & 109 & 111 & \begin{tabular}{|l|}
113 \\
\end{tabular} & 113 & \begin{tabular}{|l|}
113 \\
\end{tabular} & 111 & \begin{tabular}{|l|}
113 \\
\end{tabular} & 126 & \\
\hline \multirow[t]{3}{*}{$\overline{\text { ERI }}$} & $\mathrm{Og}$ & -.082 & .027 & 101 & .024 & $.168^{2}$ & .186 & \begin{tabular}{|l|l|}
.181 \\
\end{tabular} & $-.259^{\prime}$ & .002 & .076 & .147 & $.209^{\circ}$ & \begin{tabular}{|l|}
192 \\
\end{tabular} & $346^{\circ}$ & 1.000 \\
\hline & Sif & .191 & .382 & 134 & .398 & .038 & .020 & \begin{tabular}{|l|}
.032 \\
\end{tabular} & .003 & .490 & .214 & .063 & .014 & \begin{tabular}{|l|l}
.022 \\
\end{tabular} & \begin{tabular}{|l|}
.000 \\
\end{tabular} &. \\
\hline & $\overline{\mathrm{N}}$ & 115 & 123 & 123 & 116 & 112 & \begin{tabular}{|l|}
121 \\
\end{tabular} & 106 & 108 & 110 & 110 & 110 & 109 & \begin{tabular}{|l|}
110 \\
\end{tabular} & \begin{tabular}{|l|}
117 \\
\end{tabular} & \begin{tabular}{|l|}
123 \\
\end{tabular} \\
\hline
\end{tabular}


Multiple logistic regression analysis was conducted to examine predictors of prediabetes as outlined by Hosmer and Lemeshow (2000). The recommended steps are as follows: 1) perform initial bivariate analyses of the relationship of the dependent variable to individual predictor variable candidates, 2) select the predictors that have a bivariate association with the dependent variable at $p<0.25$ as candidates for main effects within a multiple logistic regression model, 3) evaluate the contribution of each predictor to the multiple logistic regression model by reviewing $p$ values, and 4) check for scientifically justified interactions among the predictors.

Using steps outlined by Hosmer and Lemeshow (2000) for building an initial model, a series of bivariate logistic regressions between the significant predictors and the dependent variable (prediabetes) were calculated. Steps one and two were completed as follows: bivariate logistic analysis followed by candidate selection $(p<0.25)$. Bivariate logistic regression analysis was done with prediabetes as the dependent variable and high density lipids, triglycerides, systolic blood pressure, diastolic blood pressure, body mass index, waist circumference, coping, dietary habits, exercise, alcohol, smoking, insomnia, job strain, and job imbalance as the predictors. Six predictors (triglycerides, body mass index, waist circumference, alcohol, insomnia, job imbalance) were statistically significantly associated with prediabetes as indicated by $p<0.25$. A summary of the predictors derived from the initial model can be found in Table 17.

Table 18. Predictors Selected as Candidates for Main Effects.

\begin{tabular}{l|l|l} 
Predictor & $\left.\boldsymbol{\chi}^{\mathbf{2}} \mathbf{1}\right)$ & $\boldsymbol{p}$ value \\
\hline Triglycerides & 2.06 & 0.143 \\
\hline Body Mass Index & 5.79 & 0.017 \\
\hline Waist Circumference & 4.60 & 0.033 \\
\hline Alcohol & 7.71 & 0.005 \\
\hline Insomnia & 2.76 & 0.095 \\
\hline Job Imbalance & 2.76 & 0.110
\end{tabular}


Next, each of the six significant predictors was evaluated for contribution to the multiple regression model and $p$ values were reviewed for significance $(p<0.05)$. A backwards elimination procedure was used as recommended by Royston and Sauerberi (2008). Backwards elimination starts with the full model and eliminates only variables with little influence on the model fit. In this step, the multiple logistic regression analysis conducted to predict prediabetes using the six significant predictors (triglycerides, body mass index, waist circumference, alcohol, insomnia, job imbalance) indicated that alcohol, insomnia, and job imbalance significantly increased participants' risk of prediabetes $\left(\chi^{2}(4)=16.82, p<0.05\right)$. The odds ratio for alcohol was 0.20 (95\% CI, 0.638 - 0.643), indicating that for every one-unit increase in alcohol, participants were 0.2 times as likely (20\% less likely) to have prediabetes. The odds ratio for insomnia was $1.14(95 \% \mathrm{CI}, 1.03-1.26)$, indicating that for every one-unit increase in insomnia, participants were 1.14 times as likely (14\% more likely) to have prediabetes. The odds ratio for job imbalance was 0.05 (95\% CI, $0.003-0.895)$, indicating that for every oneunit increase for job imbalance, participants were 0.05 times as likely (5\% less likely) to have prediabetes.

\section{Mediation of Relationship Between Work Stress and Prediabetes}

Analysis of the data showed that only one measure of work stress, job imbalance, was statistically significantly related to prediabetes. The mediation effect of health behaviors on the relationship between job imbalance and prediabetes in full-time employees was assessed through a mediation analysis using structural equation modeling. The purpose of a mediation model is to provide a mathematical explanation for how or why two variables are related when a mediating variable is hypothesized to mediate the relationship (Fairchild \& McKinnon, 2009). Structural equation modeling (SEM) uses a 
hypothesis-testing approach that uses conceptual paths, path diagrams, or linked regression-like equations to capture observable and latent constructs or complex relationships among observed variables (Gunzler et al., 2013). The SEM model tests the extent to which a hypothesized model adequately describes the data.

There are four important aspects of fitting hypothesized models into SEM: 1) the rationale for which the model-fitting process is based, 2) the statistical significance, 3) the estimation process, and 4) the goodness-of-fit statistic (Byrne, 2012). For the model fitting process, the researcher specifies a model then uses the data obtained from the sample to test the model (Figure 2). The null hypothesis being tested by the model states that the hypothesized model represents the population. The goal is to accept the null hypothesis. Statistical significance is determined at alpha levels below 0.05 . The focus of the SEM estimation process is to yield parameter values such that the discrepancy between the sample the covariance matrix and the population covariance matrix implied by the model is minimal.

SEM calculates several goodness-of-fit values, which relate to the model as a whole. The aim is to reproduce variance-covariance differences of the model. A summary of SEM goodness-of-fit statistics can be found in Table 18. Goodness-of-Fit Statistics refer to the ability of the model to reproduce the variance-covariance matrix. The Comparative Fit Index is used to compare two models. It can be used to compare the saturated model to the non-saturated model. A saturated model perfectly reproduces all of the variances, covariance and means of the observed variables and has the best fit possible. A saturated model will yield a chi-square $(\chi 2)$ of zero. An unsaturated or baseline model is a model that includes the means and variances of all observed variables plus the covariances of all observed latent variables. The Tucker-Lewis Index is an incremental measure of fit where the best model is defined by the null model. The Root 
Mean Square Error of Approximation (RMSEA) is an absolute measure of fit based on the non-centrality parameter and is reported most often. The Chi-Square Test of Model Fit $(\chi 2)$ represents the discrepancy between the unrestricted sample covariance matrix and the restricted covariance matrix. For models with $75-100$ cases, $\chi^{2}$ is generally a good measure of fit (Byrne, 2012; Kenny, 2015).

Table 19. Goodness of Fit Indices for SEM Model.

\begin{tabular}{|c|c|c|c|}
\hline Index & Abbreviation & Value & $\begin{array}{l}\text { Criteria for } \\
\text { acceptable fit }\end{array}$ \\
\hline Comparative Fit Index & CFI & 1.000 & $\geq 0.95$ \\
\hline Tucker Lewis Index (Non-Normed) & TLI & 1.007 & $\geq 0.95$ \\
\hline Standardized Root Mean Square Residual & SRMR & 0.036 & $\leq 0.08$ \\
\hline Root Mean Square Error of Approximation & RMSEA & $<0.001$ & $\leq 0.06$ \\
\hline 90\% Confidence Interval around RMSEA & $90 \% \mathrm{CI}$ & $0.000-0.035$ & Upper bound $<0.08$ \\
\hline P value for $H_{0}:$ RMSEA $<=0.05$ & $\mathrm{P}_{\text {CLOSE }}$ & 0.991 & $>0.05$ \\
\hline Chi-Square Test of Model Fit (P Value) & $\mathrm{P} \chi^{2}$ & 0.779 & $>0.05$ \\
\hline
\end{tabular}

The SEM model tested the hypothesized mediation effects of sleep on the relationship between job imbalance and prediabetes (Figure 2). The hypothesized mediation model assumes that: 1) the effect of an independent variable on a dependent variable is mediated by a mediating variable and 2) the independent variable still affects the dependent variable. In other words, the assumption is that there is complete mediation in the variables whereby the independent variable no longer affects the dependent variable after the mediator has been controlled (Barron \& Kenny, 1986).

Barron and Kenny (1986) procedures outline steps required for testing mediational hypotheses. The steps are as follows: Step 1) the independent variable is correlated with the dependent variable, Step 2) the independent variable is correlated with the mediator, Step 3) the mediator variable and the dependent variable are correlated, and 
Step 4) the effect of the independent variable on the dependent variable is zero after controlling for the mediator variable. If all four steps are met, the data are consistent with complete mediation. If only Steps 1-3 are met but not Step 4, then a partial mediation is indicated. Each of the steps was followed using SEM. Interpretation of results is reported below.

Figure 2. The hypothesized model of mediation.

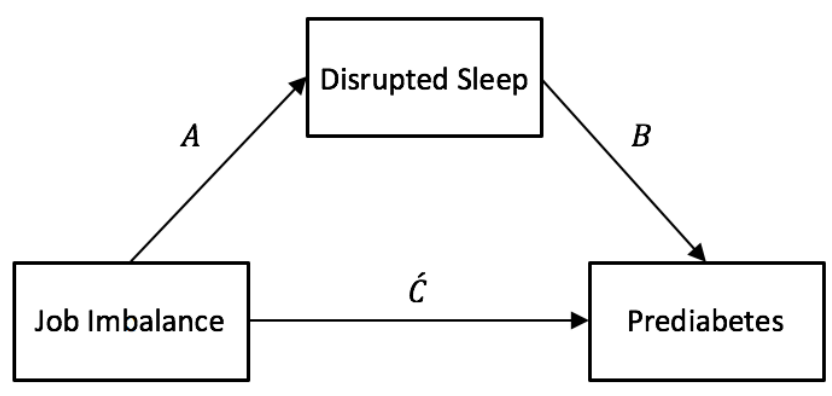

Step one tested the relationship between job imbalance and prediabetes (path C). Although Spearman's rho correlation results showed a statistically significant relationship between job imbalance, and prediabetes, the SEM results indicated that there was no statistically significant relationship between job imbalance and prediabetes, $\beta=$ $0.15, \mathrm{SE}=0.092, p=0.09(95 \% \mathrm{CI}-0.334-0.027)$. Step one criteria were not met. Goodness-of-Fit Statistic RMSEA $=0.00, p<0.05, \chi 2=0.00$. The subsequent steps were no longer necessary. The SEM mediation model indicated that sleep did not significantly mediate effect the relationship between job imbalance and prediabetes.

\section{Coping as a Mediator}

Analysis of the data showed that job imbalance was not statistically significantly associated with prediabetes. However, as stated above, a mediation effect can still be present. However, when a structural equation modeling test was used to determine the mediation effects of coping on the relationship between job imbalance and prediabetes, 183 
the test of mediation effects showed that coping did not statistically significantly mediate the relationship between job imbalance and prediabetes.

\section{SUMMARY}

Results of this study were able to answer the research questions and yielded significant findings. The prevalence rate of prediabetes was much lower than the

prevalence in the US population. Participants in this study were mostly young, White males with a college education working in an engineering or technical role. Approximately half of the participants had abnormal BMI, but most had waist circumference measurements that were within recommended US guidelines. None of the participants had dyslipidemia. Most had normal blood pressure. Only one-third of the participants reported compliance with USDA dietary guidelines, however, two-thirds reported exercising regularly. Most participants did not smoke and most drank alcohol at least occasionally. More than one-third of the participants reported disturbances in sleep. Nearly all of the participants reported work stress (job strain or job imbalance). Most participants reported using avoidant-type coping efforts.

The analyses yielded some significant relationships among the variables. Job strain and job imbalance were found to be significantly related. Job strain was not significantly related to prediabetes, but job imbalance was found to be significantly related to prediabetes. Both job strain and job imbalance had an inverse relationship with prediabetes. The Perceived Stress Scale was significantly related to both job strain and job imbalance. Three variables were significant predictors of prediabetes: low alcohol use, sleep disturbance, and low job imbalance. No significant mediators between job imbalance and prediabetes were found in this study. 
Findings from this cross-sectional, exploratory, descriptive study contributed to nursing science through a comprehensive analysis of risk factors for prediabetes and relationships between and among those variables in a worker population of a single, computer technology company located in central Texas. Results of this study provide details about prediabetes risk in this participant sample. Results identified that participants eat poorly; experience global and work stress; and have difficulty sleeping. Study findings can provide a framework for education, intervention, and counseling regarding risk for prediabetes. Findings can also guide worksite interventions aimed at reducing work stress from an organizational perspective. 


\section{Chapter Five}

\section{DiscuSSION AND CONCLUSIONS}

The purposes of this descriptive, cross-sectional study were to: (1) describe the prevalence of prediabetes (dependent variable) and work stress (job strain and job imbalance) in employees at a large employer in the Professional, Scientific, and Technical Services sector headquartered in central Texas; (2) describe the relationship between stress, work stress, and prediabetes; (3) explore the potential interactive effects of socio-cultural factors, stress, work stress, health behaviors, and coping on prediabetes; and (4) explore the potential mediating effects of health behaviors and coping on the relationship between work stress and prediabetes. The Conceptual Model for Metabolic Risk framework was developed specifically for this study. The model, Conceptual Model for Metabolic Risk, is situated within a contextual environment, employment at the computer technology company, the setting for this study. The model was used to organize the relationships between and among the dependent variables and their relationship with the dependent variable. A convenience sample of 133 employees was recruited to participate in this exploratory study. This chapter is organized in two sections, discussion and conclusion, and implications and recommendations. The discussion section is organized by the research questions. A summary of the study's findings can be found on Table 16.

\section{Discussion of the Findings}

Prevalence of Prediabetes. It is estimated that $37 \%$ of US adults have prediabetes (ADA, 2015). The results of this study showed a much lower rate of prediabetes (19\%). Risk of prediabetes increases as age increases, especially in people over 65 (CDC, 2014). The mean age in the participant sample was 37.2. Higher body mass also increases the 
risk for prediabetes. As body mass increases, risk for prediabetes increases. In the US population, approximately $2 \%$ of individuals within the normal body mass index (BMI) range have prediabetes. Nearly $4 \%$ of individuals in the overweight BMI range and approximately $6 \%$ of individuals in the obese BMI range have prediabetes (Rolka, et al., 2008). Results in this study showed that approximately half (48.8\%) of the participants were in the normal BMI range. Less than one third of participants were in the overweight BMI $(28.5 \%)$ or obese BMI $(21.95 \%)$ range. The prevalence of prediabetes was $19 \%$ in the participant sample within this study. It is possible that the participants' younger age and leaner BMI reduced their overall risk for prediabetes, and, therefore, could account for the lower prevalence rate. It is likely that the lower risk for prediabetes accounts for the lower prevalence of prediabetes in this participant sample.

\section{Prevalence of Job Strain.}

It is estimated that up to $80 \%$ of US workers experience stress at work and approximately $40 \%$ report their job to be very or extremely stressful (NIOSH, 1999). The definition criteria for job strain that individual participants reported $41 \%$ job strain when defined by the within sample median and $52 \%$ when defined by national means. The results of this study showed a high report of work stress as calculated by the job strain ratio term within the participant sample (92\%), indicating a prevalence rate of $92 \%$. The CDC recognizes that individual differences may partially explain the experience of work stress (NIOSH, 1999). There are some important individual differences between the study participants and the US worker population. By comparison, the participants in this study were younger and more educated than the typical US worker. The median age of US workers is 41.9 , whereas the median age for the participants was 37.2 . Over $90 \%$ of the US workforce has a high school diploma; all of the participants had a high school 
diploma or higher. Approximately half (53.1\%) of US workers are male, however, $67.7 \%$ of the participants were male. In comparison to the US worker population, the participants were younger, more educated, and more likely to be male. However, these differences do not account for the higher reports of job strain. Typically, younger and more educated workers experience less job strain than older or less educated workers (Feinstein et al., 2006; Marmot et al., 1978; Marmot and Smith, 1991; vonBornsdorff et al., 2011). Female workers typically experience more work stress than male workers (Webster and Bergman, 1999). In addition to demographic similarities, the participants were similar in job type and number of work hours per week. Nearly $70 \%$ of participants worked engineering or technical job roles. Nearly all the participants worked 40 hours per week. Based on these findings, it is likely that individual differences do not account for the increased report of job strain in the participant sample in this study. However, work conditions also contribute to the experience of job strain (NIOSH, 1999) and could account for the higher prevalence of job strain in the participant sample.

The CDC states that certain work conditions will result in work stress for most people. Work conditions such as work roles, career concerns, interpersonal relationships, excessive work demands, and conflicting expectations are the primary reason for work stress (NIOSH, 1999). Sources of work stress related to work conditions include: Low salaries (47\%), lack of opportunity for growth and advancement (44\%), heavy workload (39\%), long hours (38\%), and undefined work expectations (36\%) are sources of work stress in the US worker (APA, 2013).

Participants in this study were employed in white collar jobs such as management, office/administration, or engineering/technical roles. The JCQ measured job strain as a measure of work stress. Job strain is the interaction effect of job demands and decisional latitude Job strain accounts for both work demands and work conflict. Participants mean 
scores on the job demands scale were lower than the national mean scores, suggesting lower job demands in this sample $(M=29.8, S D=4.43$ vs. $M=30.9, S D=8.48$, respectively); however, mean scores for the decisional latitude scale were much lower in the participants' scores compared to national means, suggesting lower decisional latitude $(M=47.39, S D=5.6$ vs. $M=70.3, S D=15.6$, respectively $)$.

The decisional latitude score is derived from two subscales: skill discretion and decision-making authority. The participants' scores on the skill discretion scale $(M=$ $16.55, S D=2.38)$ and the decision-making authority $(M=30.89, S D=4.11)$ were lower than national mean scores $(M=33.5, S D=8.5$ and $M=36.8, S D=9.90$, respectively $)$. The skill discretion mean score was very low compared to national means. Skill discretion is defined as the opportunity to use and develop skills on the job. According to the WHO, working in a job role that is mismatched to a worker's skills, knowledge, and abilities is the primary reason for experiencing work stress (Leka \& Jain, 2010).

The participants' job role may have also contributed experience increased work stress. Although, historically, studies have shown that blue collar workers are more likely to experience job strain (Marmot et al., 1978; Marmot and Smith, 1991), white collar workers in technical and administrative support roles have a higher rate of neurotic response to stress than their counterparts in blue collar jobs ( $R R=1.6$ vs. 1.1, respectively) (Webster \& Bergman, 1999). Workers employed in a technical industry have been shown to have high reports of job strain due to heavy work load and role ambiguity (Dhar \& Dhar, 2010). It is likely that the higher reports of job strain within the participant sample were related to work conditions. 


\section{Prevalence of Job Imbalance}

Forty percent of US workers report their work to be extremely stressful (NIOSH, 1999). Up to $80 \%$ of workers report experiencing work stress. The results of this study showed that more than nearly two-thirds (64\%) of the computer company employees reported work stress as measured by high job imbalance and about a third (34.1\%) reported work stress as measured by low job imbalance within the participant sample. The WHO states that work related stress is the response to work demands or pressures that are not well matched to a worker's knowledge or ability to cope with stress. Work related stress hazards can be divided into work content and work context (Cox et al., 2000; Leka \& Jain, 2010). The ERI scale measures work content and context by measuring worker effort and reward received in turn. The ratio between perceived effort and reward calculates the extent of work stress when measured as job imbalance. Job imbalance results when effort exceeds rewards. Job imbalance can result in increased risk for emotional exhaustion, physical disorders or symptoms, and job dissatisfaction (deJonge et al., 2000).

The effort scale measured perceived extrinsic effort. Higher scores on the effort scale indicate more perceived extrinsic effort. Median perceived effort scores in the participant sample were lower than the scale median score (12 vs. 15, respectively), suggesting that participants in this sample perceived their work to have relatively low extrinsic effort. The reward scale measured perceived occupational rewards received by the worker. Higher scores indicate a greater number of rewards received. Median perceived reward scores in the participant sample were lower than the scale median score (31 vs. 33, respectively), suggesting that participants in this study received fewer occupational rewards for their work. Although the participants in this study reported relatively lower effort, the amount of reward received in turn was also lower. Individuals 
working within the technical industry have been reported to have excess time pressure, role ambiguity, role conflict, and low rewards (Dhar \& Dhar, 2010). Perceived rewards that are lower than expected may lead to higher mental health disorders in white collar workers (Ndjaboué et al., 2014), whereas occupational rewards such as financial stability, job security, and work-related self-esteem serve to buffer the effect of work stress (Sembajwe et al., 2012; Siegrist, 1996). It is likely that the higher reports of job imbalance by the participants in this study were related to fewer perceived rewards.

\section{Job Strain and Job Imbalance}

The WHO classification of work stress into work content and work context can also be described as interactional stress and transactional stress. Interactional stress is the experience of stress that is directly related to interactions with structural or physical hazards in the work environment, such as noise, and is more closely aligned with work content. Transactional stress is the cognitive or emotional stress resulting from personenvironment interactions and is more closely aligned with work context. Interactional stress has a direct effect on health, while transactional stress has an indirect effect (Leka \& Jain, 2010).

Historically, two models have been the most influential in describing work stress and its relationship to health, Karasek's Job Control Demand Model and Siegrist's Effort Reward Imbalance Model (Leka \& Jain, 2010). The Job Demand Control Model (JDC) and the Effort Reward Imbalance (ERI) models measure work stress through job strain and job imbalance, respectively. Job strain has two intersecting dimensions, job demands and decisional latitude, which have an interaction effect. Job imbalance is the proportionate effect of two experiences, perceived effort and perceived rewards. Each scale captures measures of interactional stress (physical environment, work pace) and 
transactional stress (decision-making authority, interpersonal work relationships). Studies investigating the relationship between work stress and health have often included both the job strain and job imbalance questionnaires, either in whole or in part, to measure work stress (Gilbert-Oimet et al., 20061 deJonge et al., 2000; Kumari et al., 2004; Ota et al., 2005). The concurrent use of the job strain and job imbalance measures suggests that each scale captures a unique aspect of work place stress.

It is also possible that the job strain and job imbalance measures have some convergence. In a study by Sembajwe et al. (2012) correlations between psychosocial measures of work stress in job strain and job imbalance questionnaires were investigated. Using Spearman correlations, results indicated that measures from the ERI and the JDC were significantly correlated. The effort measure from the ERI scale and the psychological demand measure from the JDC scale were highly correlated ( $Q=0.55, p<$ 0.001) as well as the reward measure from the ERI scale and the social support measure from the JCC scale ( $\varrho=0.42, p<0.001)$. Similarly, Reineholm, Gustavsson, and Ekberg (2010) investigated the correlation between the ERI and JDC measures. The job demands variables $(r=0.69, p<0.001)$ and social support $(r=0.55, p<0.001)$ in the job strain questionnaire were correlated with effort variables in the job imbalance questionnaire. Results of their study showed that job strain and job imbalance were statistically significantly related $(\varrho=0.346, p=0.00)$. The findings in this study are congruent with these two studies.

Results within the participant sample in this study suggest that experiences of job strain and job imbalance were similar. Job strain and job imbalance were statistically significantly related within the participant sample in this study $(\mathrm{Q}(115)=0.346, p<$ $0.00)$. It is possible that the homogenous work environment contributed to the overlap in work stress measures. All of the participants in this study were white collar workers 
within a single computer technology company. It is likely that the work environment and work culture experiences were similar across employees, and, therefore, work stress experiences may also be similar.

\section{Job Strain and Prediabetes}

Historically, studies related to job strain have studied the relationship between job strain and cardiovascular disease (Fujino et al., 2006; Karasek \& Theorell, 1998; Landsbergis et al., 2008; Marmot et al., 1978Schnall et al., 2000; Vrijkotte et al., 2000). A few studies have explored the relationship between job strain and components of prediabetes such as glucose metabolism (deBacquer et al., 2009; Karlsson et al., 2003; Suwazono et al., 2009) and insulin resistance (Chandola, Brunner \& Marmot, 2006). Limited empirical evidence suggests that a component of job strain (shift work) is related to prediabetes (deBacquer, 2009; Suwazono, 2009).

One aim of this study was to explore the relationship between job strain and prediabetes as an outcome variable. Although nearly all of the participants reported job strain, most did not have prediabetes. The prevalence rate for job strain in this study was $92 \%$ while the prevalence rate for prediabetes was $19 \%$. Job strain and prediabetes were not statistically significantly related $(\varrho(111)=-0.94, p=0.16)$. The lack of significant association is likely due to the lack of prediabetes prevalence. It is interesting to note, however, the direction of the relationship between job strain and prediabetes, which has a negative association.

Although not statistically significant, results of this study suggest that job strain and prediabetes may be inversely associated. The results of this study contradict previous studies that suggest that high job strain is most likely to be related to untoward health outcomes. Low job strain has not been associated with poor health outcomes. However, 
characteristics of lower stress jobs may offer some insight. In their hallmark book, Karasek and Theorell (1990) theorize that low job strain can result from job roles in which there is passivity, lack of motivation, or complacency. These experiences may affect participation in health-promoting behaviors (Kuovonen et al., 2007; vonBonsdorff et al., 2012). Lack of participation in health promoting behaviors can lead to poorer health outcomes.

It is beyond the scope of this study to investigate whether participants in this study with lower job strain lacked motivation, were passive, or complacent in their work positions. It may be useful to include measures of attitude toward job roles, such as passivity or complacency, in future studies.

\section{Job Imbalance and Prediabetes}

Siegrist's Effort-Reward-Imbalance model is relatively new. The model was developed in 1994. As with job strain, many studies initially explored the effects of job imbalance on cardiovascular disease (Siegrist, 1994; Siegrist et al., 1997). Two studies have suggested that job imbalance is related to glucose metabolism (Li et al., 2013; Xu et al., 2012). One aim of this study was to explore the relationship between job imbalance and prediabetes as an outcome variable.

Many of the participants reported job imbalance, yet nearly all did not have prediabetes. The prevalence rate for high job imbalance was $64 \%$ and $34 \%$ for low job imbalance in this study, while the prevalence rate for prediabetes was 19\%. Spearman's rho correlation results indicated that job imbalance and prediabetes were statistically significantly, inversely related $(\varrho(109)=-0.192, p=0.022)$. However, the relationship was not significant using structural equation modeling $(\beta=-0.15, \mathrm{SE}=0.092, p=0.09$, $95 \%$ CI $-0.334-0.027)$. The Spearman's rho correlation findings from this study 
contradict previous empirical evidence that suggests job imbalance to be correlated with glycated hemoglobin (Xu et al., 2012) and Type 2 diabetes mellitus (Li et al., 2013). The results of this study suggest that low job imbalance is related to prediabetes. It is unclear to what degree job imbalance is inversely related to prediabetes in this study. It is possible that the measure of job imbalance (effort reward ratio) did not fully capture the participant's work stress experience.

An independent measure, over-commitment, a coping pattern in Siegrist's model, can be measured along with the job imbalance questionnaire. The over-commitment scale was not included in this study, as inclusion of the scale was not required to calculate job imbalance. In previous, larger studies, reports of over-commitment were associated with increased risk of cardiovascular disease (Bosma et al., 1998) and lower overall well being (deJonge et al., 2000). It is not possible to determine whether over-commitment influenced the results of this study. Seigrist and Marmot (2006) state that imbalance is likely to occur in workers who either have no alternative choice for work or are in highly competitive jobs. It is not possible not to know whether these workers were experiencing these factors. Including the over-commitment scale and questions addressing competitive work environment in future studies may offer more insight.

\section{Job Strain and Stress}

In developing the Demand-Control Model (Job Strain) of work stress, Karasek and Theorell (1990) used Selye's General Adaptation Model of stress as a comparison and benchmark. The General Adaption Model (GAM) was chosen as the general model of stress and the stress response. Karasek and Theorell (1990) concluded that there are some common estimates between the two models. The GAM depicts stress as a continuum between eustress and distress. The Demand-Control Model (DCM) is a two- 
dimensional representation of the continuum between low job strain and high job strain. In comparing the models, Karasek and Theorell (1990) overlaid the two models and determined that, at the point of high demand and low control, the GAM continuum and the DCM continuum yield the same prediction between physiological and psychological stress and possible disease. Based on Karasek and Theorell's conclusions, it is reasonable to conclude that measures of general stress and work stress overlap.

The current study included the Perceived Stress Scale (PSS) as a measure of global and event-specific stress. The Job Content Questionnaire (JCQ) was used to measure job strain as a measure of work stress. Previous studies have not compared the relationship between the PSS and JCQ nor its comparative outcome effects on health. However, studies have shown that general stress and work stress have a similar effect on health.

As stated previously, job strain is related to poor health outcomes, including abnormal glucose metabolism (prediabetes). In a study by Williams et al. (2013) measures of perceived stress and objective stress were used to analyze the relationship between psychosocial stressors and abnormal glucose metabolism. The PSS was used to measure perceived stress and a stressful life events scale measured objective stress. Results indicated that perceived stress and prediabetes were significantly related (OR $1.48,95 \%$ CI, 1.08-2.02). In a similar study, Eriksson et al. (2010) tested associations between stressful life events and metabolic syndrome, which includes abnormal glucose metabolism. A 15-item stressful life events questionnaire was used to measure stress. Results showed that participants who reported extremely stressful life events in financial or work-related domains had higher odds of metabolic syndrome diagnosis (OR 2.80, 95\% CI, 1.69-4.63, $p=0.001$; OR 1.79, 95\% CI, 1.17-2.75, $p=0.007$, respectively). 
Based on empirical evidence and Karasek and Theorell's conclusions, it is possible that global and event-specific stress and job strain are inter-related.

Analyses in this study suggest that the measure global and event-specific stress (PSS) may include work stress. The mean PSS score within participant sample in this study was $23.89(S D=6.44)$, indicating a moderate degree of global and event-related stress. Most of the participants (92\%) reported job strain. Results showed that scores on the PSS and the JCQ were significantly statistically related within participant sample $(\mathrm{Q}(121)=0.337, p<0.001)$, suggesting that reports of global or event-specific stress were related to reports of job strain.

\section{Job Imbalance and Stress}

Although short-lived or infrequent episodes of stress pose little threat to health, chronic stress can lead to psychological or physical ill health. Work stress includes acute and chronic stress. It is the position of NIOSH that work stress is most often caused by work conditions, however, individual and situational factors can exacerbate or attenuate work stress. The NIOSH Model of Job Stress outlines a causal pathway between stressful job conditions and risk of illness. This pathway is not direct, however. Instead, it includes individual and situational factors as potential mediators or moderators between stressful work conditions and risk of injury or illness (NIOSH, 1999). Individual or situational factors can protect (mediate) or aggravate (moderate) the effect of stressful work conditions on health. Like the NIOSH model, Siegrist's Model of Effort-RewardImbalance (ERI) (1996) includes individual and situational factors in its measurement of job imbalance. The ERI measures of individual and situational factors can mediate or moderate the effects of job imbalance on health or illness. 
In developing the ERI, Siegrist (2012) aimed to understand and explain the contribution of social and psychological stress factors to health and illness. The underlying premise of the model is that balanced social reciprocity (social exchange) is the key to positive emotion and subsequent health. Imbalance, or failed reciprocity, elicits negative emotions and leads to stress (Seigrist, 2012). The stress effects of job imbalance cause sustained strain to the autonomic nervous system and impairs self-regulation. Illness is the result of the persistent stress reactions elicited by job imbalance. Siegrist and Marmot (2006) postulate that an employee's need for approval or esteem leads to susceptibility for exhaustion and adaptive breakdown through hyperactivation of the medullary prefrontal cortex, anterior cingulate cortex, and dorsolateral prefrontal cortex. The medullary prefrontal and anterior cingulate cortex regulate blood pressure and heart rate. Hyperactivation may result in increased blood pressure and heart rate, which in turn, causes metabolic stress. The dorsolateral prefrontal cortex is responsible for cognitive flexibility and planning. Hyperactivation may reduce flexibility and planning decisions. Based on Seigrist's explanation of the stress experienced with job imbalance, it can be concluded that job imbalance triggers the stress response.

Like the experience of job imbalance, acute or chronic stress activates the autonomic nervous system and the stress response (Vedhara \& Irwin, 2007). Repeated or chronic stress response activation can overburden the autonomic nervous systems and lead to illness (Grundy, 2012; Wellen \& Hotamisligil, 2005). Also, the experience of job imbalance and general stress translates into similar symptoms. The experience of job imbalance and stress can result in symptoms such as: headache, sleep disturbances, difficulty concentrating, short temper, upset stomach (NIOSH, 1999), or tense muscles, increased heart rate or blood pressure, cardiovascular inflammation, increased glucose, increased cortisol, improper diet, and poor digestion (APA, 2014). The activation of the 
stress response and the consequent stress-related symptoms can lead to altered glucose metabolism (prediabetes) through a variety of pathways (Abdul-Ghandi, 1999; Lloyd et al., 2014; Li et al., 2013).

The current study used the Perceived Stress Scale (PSS) as a measure of global and event-specific stress. The Effort-Reward-Imbalance Questionnaire (ERIQ) was used to measure job imbalance as a measure of work stress. Previous studies have not compared the relationship between the PSS and ERIQ nor its comparative effects on health outcomes. However, evidence has shown that the effects of chronic stress on impaired glucose metabolism (prediabetes) and the effects of job imbalance on prediabetes are similar.

Evidence has shown that altered glucose metabolism is the consequence of inflammation initiated by physiologic response to chronic stress. Chronic metabolic overload resulting from chronic activation of the stress response and subsequent inflammation is responsible for altering glucose metabolism (Wellen \& Hotamisligil, 2005). In a study by Pyykkönen et al. (2010) the aim was to test the associations between stressful life events and development of metabolic syndrome, which includes altered glucose metabolism. A 15-item stressful life events questionnaire was used to evaluate general stress. The authors reported that the odds for developing metabolic syndrome were significantly higher in participants experiencing extremely stressful life events, especially in financial (OR 1.60, 95\% CI, 1.07-2.39, p=0.023), work-related (OR 1.79, 95\% CI, 1.17-2.75, $p=0.007$ ) or health-related (OR 1.59, 95\% CI, 1.11-2.28, $p=0.012$ ) domains. Empirical evidence suggests that stress is associated with prediabetes. Job imbalance, a type of work stress, has also been associated with prediabetes.

Schmidt et al. (2015) examined the relationship between job imbalance and metabolic syndrome in male Jordanian workers. The ERIQ was used to measure job 199 
imbalance. Fasting blood glucose was used to determined altered glucose metabolism. Results of the study showed that job imbalance was associated with metabolic syndrome $(\mathrm{OR}=1.14,95 \% \mathrm{CI}, 1.03-1.26, p=0.01)$ as well as components of job imbalance, effort and reward $(\mathrm{OR}=1.12,95 \% \mathrm{CI}, 1.00-1.25, p=0.049$ vs. $\mathrm{OR}=0.92,95 \% \mathrm{CI}, 0.84-1.00$, $p=0.05$, respectively). In a study by Li et al. (2013), the association between work stress with diabetes and prediabetes was examined. Work stress was measured with the ERIQ. The analyses showed that high job imbalance was related to a $26 \%$ higher odds ratio of prediabetes in men, even after adjusting for confounding variables $(\mathrm{OR}=1.29,95 \% \mathrm{CI}$, $1.03-1.61, p<0.05)$. The researchers concluded that repeated or continuous activation of the stress response predisposes the worker to prediabetes due to the paradoxical state of high metabolic demands without corresponding needs (Li et al., 2013). It is likely that the experience of stress within the experience of job imbalance is similar to the experience of general stress, and it is also likely that both job imbalance and general stress have comparable effects on health.

In developing his model, Siegrist explains that job imbalance and the stress response are related. Empiric evidence also suggests that job imbalance and stress are related. The results of the analysis in this study were congruent with Siegrist's explanation of the job imbalance model and with empiric evidence. Analyses indicated that job imbalance and stress were statistically significantly related within the participant sample in this study $(\mathrm{Q}(119)=0.261, p=0.002)$.

\section{Predictors of Prediabetes}

Historically, age, poor diet, sedentary lifestyle, overweight or obese body mass, and higher than recommended waist circumference have been identified as predictors for prediabetes and the consequent onset of T2DM (ADA, 2014). Bivariate correlation 
analyses in this study were congruent with findings in previous studies that showed the relationship between age, poor diet, sedentary lifestyle, high body mass, and high waist circumference and prediabetes. In this study, bivariate correlation analyses also showed a relationship between alcohol, sleep difficulty, and job imbalance and prediabetes. Logistic regression analyses indicated that alcohol, sleep difficulty and job imbalance were significant predictors of prediabetes. Each is discussed below.

Results of this study showed that use of alcohol significantly increases the likelihood of prediabetes $\left(\chi^{2}(4)=16.82, p<0.05\right)$ The odds ratio for alcohol was 0.20 (95\% CI, $0.638-0.643)$ indicating that for every one-unit increase in alcohol participants were 0.2 times as likely to have prediabetes. Previous studies have shown a u-shaped relationship between alcohol use and Type 2 diabetes mellitus. These studies suggest that drinking too little or too much alcohol is detrimental to health, but drinking alcohol in moderation has health benefits (Djousse et al., 2007; Howard, Armsten \& Gourevitch, 2004). Using a prospective design, Cullman et al. (2012) found that high alcohol use was associated with risk of prediabetes in a Swedish population, with a family history of T2DM. No studies in the US have found a relationship between use of alcohol and risk of prediabetes. The regression analysis results in this study are novel and unique to this study.

This study found that sleep difficulty significantly increases the likelihood of prediabetes $\left(\chi^{2}(4)=16.82, p<0.05\right)$. The odd ratio for sleep difficulty was $0.14(95 \% \mathrm{CI}$, $1.03-1.26)$, indicating that for every one-unit increase in sleep difficulty participants were 0.14 times as likely to have prediabetes. The pathway between sleep and diabetes is still being explored, however, empirical evidence has revealed a direct relationship between sleep deprivation and altered glucose metabolism (prediabetes). Studies have shown that reduced sleep is associated with glucose intolerance, elevated blood glucose 
(Biggi et al., 2008; Buxton et al., 2010; Rafalson et al., 2012; Wu et al., 2012) or impaired glucose metabolism (Byberg et al., 2012; Gangwisch et al., 2007; Punjabi et al., 2004). During sleep two critical processes associated with glucose metabolism occur: glucose uptake is lowered and appetite is suppressed. Acute total sleep loss or a reduction of normal sleep by two hours in one week results in pro-inflammatory cytokine response and alteration of the levels of appetite-related hormones. The resultant increase in glucose levels and residual changes in appetite increase the predisposition for prediabetes (Buxton, 2010; Knutson et al., 2006; Knutson et al., 2007). Inadequate sleep duration (< 6 hours/night) disrupts metabolism and may lead to overeating, increased appetite, and preference for unhealthy foods (Buxton et al., 2010; Knutson et al., 2006; Knutson et al., 2007). The disruption in metabolism leads to decreased insulin sensitivity and decreased glucose tolerance, markers of prediabetes (Buxton et al., 2010). Poor sleep quality also disrupts metabolism and has similar effects. Poor sleep quality is significantly associated with insulin secretion and insulin sensitivity (Byberg et al., 2012). The American Diabetes Association (ADA) recognizes that changes in circadian rhythm due to temporary or chronic sleep deprivation elevates blood glucose or negatively changes in insulin secretion (Gebel, 2011). Regression analysis results in this study are consistent with previous studies that have indicated that sleep quality and prediabetes are directly related.

In this study, reports of job imbalance greater than one significantly increased the likelihood for prediabetes $\left(\chi^{2}(4)=16.82, p<0.05\right)$. The odds ratio for job imbalance was $0.05(95 \% \mathrm{CI}, 0.003-0.895)$, indicating that for every one-unit increase in job imbalance participants were 0.05 times as likely to have prediabetes. This finding is inconsistent with previous studies. High effort and low reward interact in a reciprocal manner and result in job imbalance. Job imbalance has been associated with prediabetes in previous 
studies (Li et al., 2013; Xu et al., 2012). No studies have shown an inverse relationship between job imbalance and prediabetes. The regression analysis results of this study are inconsistent with previous findings and are unique to this study.

\section{Health Behaviors as Mediators}

A test of mediation effects with structural equation modeling (SEM) did not find health behaviors as mediators between job imbalance and prediabetes.

Table 20. Summary of Study Findings

JS=Job Strain; JI=Job Imbalance; PSS=Perceived Stress Scale

\begin{tabular}{l|l}
\hline \multicolumn{2}{c}{ Prevalence Rates } \\
\hline Prediabetes & $19 \%$ \\
\hline Job Strain (JS) & $92 \%$ \\
\hline Job Imbalance (JI) & $64 \%$ \\
\hline
\end{tabular}

Spearman's rho Correlations

\begin{tabular}{l|l}
\hline $\mathrm{JS} * \mathrm{JI}$ & $\mathrm{\varrho}(115)=0.346, p<0.00$ \\
\hline $\mathrm{JS} *$ Prediabetes & $\mathrm{\varrho}(111)=-0.94, p=0.16($ n.s. $)$ \\
\hline $\mathrm{JI} *$ Prediabetes & $\mathrm{\varrho}(109)=-0.192, p=0.022$ \\
\hline $\mathrm{JS} *$ PSS & $\mathrm{\varrho}(121)=0.337, p<0.001$ \\
\hline $\mathrm{JI} *$ PSS & $\mathrm{Q}(119)=0.261, p=0.002$ \\
\hline
\end{tabular}

Spearman's rho Bivariate Correlations

\begin{tabular}{l|l}
\hline Age & $\varrho(131)=0.196, p=0.016$ \\
\hline Body Mass Index & $\varrho(124)=0.192, p=0.017$ \\
\hline Waist Circumference & $\mathrm{\varrho}(122)=0.222, p=0.007$ \\
\hline Exercise & $\varrho(107)=-2.06, p=0.016$ \\
\hline Job Imbalance & $\varrho(121)=-0.192, p=0.022$ \\
\hline Alcohol & $\varrho(122)=-0.198, p=0.014$ \\
\hline
\end{tabular}

Multiple Logistic Regression

\begin{tabular}{c|l}
\hline Alcohol & OR $=0.20, \chi^{2}(4)=16.82, p<0.05$ \\
\hline
\end{tabular}




\begin{tabular}{l|l}
\hline Insomnia & OR $=0.14, \chi^{2}(4)=16.82, p<0.05$ \\
\hline Job Imbalance & $\mathrm{OR}=0.05, \chi^{2}(4)=16.82, p<0.05$ \\
\hline
\end{tabular}

\section{Conceptual Model for Metabolic Risk}

A conceptual framework, Conceptual Model for Metabolic Risk, was developed for this study to aid in identifying variables that are related to prediabetes. The model included independent variables, relationships between and among the variables, and their relationship to the dependent variable. Prediabetes was the independent variable and was situated within the metabolic profile. Prediabetes is a common condition in the US, affecting approximately $37 \%$ of the population. Typically, persons with prediabetes have one or more metabolic changes within the metabolic profile (dyslipidemia, hypertension, overweight). The present study found that prevalence of prediabetes within the participant sample was much lower than US prevalence rates (19\% vs. 35\%, respectively). This limited the model's effectiveness in detecting relationships and interdependence. However, despite the lower prevalence of prediabetes, significant relationships within sociocultural, health behavior, and metabolic variables were identified.

There is substantial empirical evidence to show that sociocultural (age, race/ethnicity, family history), health behavior (diet, exercise), and metabolic variables (BMI, waist circumference) contribute to alterations in glucose metabolism that lead to prediabetes and subsequent T2DM. The influence of sleep, stress, work stress, and coping on prediabetes is less known. Consistent with previous studies and position statements from the ADA, sociocultural (age), health behaviors (exercise), and metabolic (BMI, waist circumference) variables were associated with prediabetes within the participant 
sample in this study. The model, therefore, was effective in identifying risk factors typically associated with prediabetes.

The present study also found significant associations between lower alcohol intake, poor sleep, job imbalance, and prediabetes. These independent variables were postulated to have a relationship with prediabetes within the model. The model was, therefore, effective in identifying relationships between these independent variables and prediabetes. Limited empirical evidence has suggested that moderate alcohol intake is beneficial to health, and that both low and high alcohol intake can be harmful to health. This suggests that findings in the present study are consistent with previous studies. Poor and disrupted sleep have been associated with untoward changes in glucose metabolism in previous studies, and the findings in this study were in agreement. Job imbalance has been associated with metabolic changes consistent with prediabetes. However, in this study job imbalance was inversely associated with prediabetes. The model proposed a direct relationship between job imbalance and prediabetes. This was an unexpected finding.

Overall, the model was vitally important in hypothesizing relationships between and among the independent variables, clustering metabolic profile changes within prediabetes, and hypothesizing the dependent variables' relationship to prediabetes. However, it should be noted that although the model provided a framework for this study in a single computer company, it is not an explanatory model for metabolic conditions. It is possible that variables that may influence metabolic conditions were either not included in the model or exist outside the ecological context, e.g., early childhood experiences or genetic predisposition.

The primary strength of this study was directly related to the conceptual model. The Conceptual Model for Metabolic Risk included a comprehensive list of independent 
variables that considered sociocultural, heath behavior, stress, coping, and metabolic variables that have been related to prediabetes in the previous studies. Another strength was the high number of participants who reported work stress. Two measures of work stress (job strain and job imbalance), a measure of global and event-specific stress, and a measure of coping were included in this study. This broad, simultaneous inclusion of stress and coping variables is unique to this study and has not been studied to date.

\section{Limitations}

The primary limitation of this study was its cross-sectional design and convenience sample in a single technology and industry company in central Texas. The cross-sectional nature of the data does not allow for causal conclusions regarding the relationship between work stress and prediabetes. Some of the instruments used in this study did not demonstrated good reliability with this population (Table 4). The comparatively low reliability coefficients in this study may affect analyses and interpretation of results. In addition, the exclusion criteria were biased toward healthy participants. By nature of the condition, people with prediabetes may have abnormal biomarkers, but disease has not yet occurred. All the theoretical variables included in the conceptual model were self-reported. Self-selection bias was another potential limitation. In addition, the sample consisted of primarily younger, White males. All the participants worked in white-collar job roles. Blue collar roles, such as sanitation or food handling jobs, were contracted to a third-party agency, thus those workers were not employees of the computer company and unavailable for participation in this study. The findings cannot be generalized to groups with differing ethnic backgrounds, females, workers in blue-collar jobs, or workers outside of central Texas. 
Another limitation was the lack of longitudinal empirical data that would explicate the pathways linking work stress and prediabetes, especially in the US. An early power analysis calculated using EpiInfo suggested that a one-tailed test with 0.90 confidence interval would require a sample of 92 participants. EpiInfo was used due to the epidemiological nature of this study and the dearth of effect size information in previous studies. Despite the 133 participants, there may have been insufficient power to detect all the dependent and interdependent relationships. Furthermore, the prevalence of prediabetes within the participant sample was low. A larger number of participants with prediabetes may have shown different results. It is plausible to conclude that a larger number of participants with prediabetes could elucidate relationships between and among the study variables.

A potential threat to internal validity was the lack of consistency in biometric testing procedures. Measurements for weight and waist measurements were taken with clothing on, which could lead to incorrect and inconsistent results. Height measurements were taken by visually inspecting the stadiometer from approximately six feet away. This could lead to incorrect measurement; a flat tool placed directly on the head that at a 45degree angle from the stadiometer is more accurate than visual observation. A single measurement of fasting blood glucose was used to confirm a prediabetes diagnosis. Although employees were to return to the Health Clinic for a second fasting blood glucose to validate initial fasting blood glucose, they opted not to make an appointment for follow-up. In addition, the Health Clinic chose to omit the glycated hemoglobin test altogether, even before lack of follow-up appointments by the employees. Another potential threat was that tests of correlation confirmed multicollinearity between the work stress and stress variables. 
Lessons learned were: 1) the importance of an adequate sample size to support the large number of variables and potential relationships, 2) future studies should include a more diverse sample of study participants, 3) the importance of strict and consistent measurement techniques.

\section{IMPLICATIONS AND RECOMMENDATIONS}

\section{Nursing Practice}

The hallmarks of nursing practice are patient assessment and patient education. Prediabetes is a multi-dimensional condition where abnormal metabolic processes are occurring but T2DM disease has not yet been diagnosed. Several metabolic changes are simultaneously occurring, including disruption in glucose metabolism and fat metabolism. Risk factors for prediabetes include many biobehavioral aspects. If no actions are taken, prediabetes can lead to T2DM in 7-10 years (ADA, 2015).

The signs and symptoms of prediabetes are often subtle and are often unnoticed. As such, nurses can provide a pivotal service to persons at risk for prediabetes. Nurses should know and understand the risk factors for prediabetes. Educating nurses about nontraditional risk factors for prediabetes through inclusion of health consequences of stress and poor sleep in nursing education curricula or through continuing education courses for nurses already in practice will increase the likelihood of identifying persons at risk for prediabetes.

The mean age of the participants with prediabetes in this study was 43.5. The ADA recommendations for prediabetes screening begin at age 45 . Nurses should be aware that risk for prediabetes may be in younger persons with and without other metabolic changes, e.g. obesity. Screening for individual experiences of stress, work stress, and sleep may help nurses identify persons at risk for prediabetes that do not fit 
into current ADA recommendations, such as persons younger than 45 or persons 45 or older who do not have metabolic risk. As such, when interacting with patients, nurses should assess for prediabetes risk from sociocultural, biobehavioral, and physiological perspectives. Nursing assessments should include questions about typical risk factors associated with prediabetes such as age, overweight, and dyslipidemia. Assessments should also include assessments for stress, work stress, and sleep. When educating patients about prediabetes, nurses should include how to manage and cope with work stress and how to establish routines to ensure adequate sleep.

Nurses can also develop worksite intervention programs aimed at reducing the risk for prediabetes. These worksite programs can include topics such as work-life balance, building and maintaining social support, keeping a positive outlook, and the importance of sleep.

\section{Nursing Research}

Future research on prediabetes should continue to explore the relationship between work stress and prediabetes, stress and coping and prediabetes, and sleep and

prediabetes. It would be especially important to include individual characteristics of workers, such as motivation, commitment, complacency, or passivity. A replication of this study with a larger, ethnically diverse sample would be valuable to see if significant relationships exist between stress, work stress, coping, sleep, and prediabetes. Purposeful sampling of persons with prediabetes may better detect relationships among and between independent variables.

At a later date, subsets of the sample will be analyzed for differences in social support, coping, and global stress. Future directions for prediabetes research should include a comprehensive analysis of stress, work stress, and coping. Biomarker measures 
of stress, such as cortisol or interleukin-6, would add to the knowledge of work stress and prediabetes. Biomarkers can be used to support reports of stress with physiologic data. Biomarkers can also be used to determine a relationship between stress and prediabetes on a physiologic basis.

Sleep and its relationship with stress, work stress, coping, and prediabetes can be further explored to determine its influence as an antecedent or consequence of stress or coping. Future studies should compare prediabetes risk factors included in the Conceptual Model for Metabolic Risk in white-collar and blue-collar workers.

\section{Worksite Implications}

Worksite wellness programs have been shown to reduce employer costs, reduce absenteeism, and increase morale (Matke et al., 2013). However, according to the RAND Employer Survey (Matke el al, 2013) worksite wellness program are not very popular. Approximately one-half of US employers offer wellness programs. Wellness programs include either wellness screening or interventions to reduce health risks and promote overall health. Screening activities generally use health risk assessments, which include surveys to assess health-related behaviors and risks, biometric data, or both. The RAND Employer Survey data showed that employers generally use data collected during health screening activities for program planning and evaluation and for assisting employees identify preventative interventions to address potential health risks.

Typically, wellness programs are aimed at disease management or lifestyle management. Disease management programs address current health conditions and illness in order to reduce long-term health effects associated with chronic illness. Lifestyle management programs are aimed at improving or eliminating poor healthrelated behaviors such as poor diet or smoking that predispose employees for illness. 
Typically, disease management programs demonstrate benefits for the employee (improved health) and employer (reduced absenteeism) in the short term. Benefits from lifestyle management programs are realized much later (Matke et al., 2013). However, less than one-half of employees in worksites that offer wellness program undergo complete health risk assessment screening. Fewer still take advantage of health risk assessment screening results. Of those identified with health risks (e.g., lack of exercise), less than one-fifth choose to participate in worksite wellness programs (Matke, 2013). It may be that worksite current health promotion and wellness programs are limited in scope.

National initiatives such as the Total Worker Health program (NIOSH, 2015) advocate for the promotion of work-site wellness programs as part of a comprehensive review of the worksite. Recommended worksite wellness interventions included in the Total Worker Health program are not only aimed at disease management and lifestyle changes but also include programs and policies aimed at improving worksite safety, worksite culture, leadership styles, and health insurance policies. The National Healthy Worksite Program (CDC, 2014) also advocates for comprehensive measures. The National Healthy Worksite Program provides worksites with toolkits to address health and wellness programs, workplace health promotion, and organizational change measures (CDC, 2016). These toolkits include step-by-step instruction on how to implement wellness programs; measure outcomes, such as productivity, health care associated costs; and support worksite leadership. Both national initiatives include a thorough review of the workplace, including organizational practices, policies about work, and promotion of health and safety of workers. By implementing multi-level change, overall health and wellness is achieved and maintained. It is possible that current worksite wellness programs have had a narrow focus (CDC, 2015; Matke, 2013; NIOSH, 2014). Employers 
should consult national resources such as the CDC and take initiative to implement comprehensive worksite initiatives to ensure and healthy and productive workforce.

\section{Policy Implications}

As previously stated, national agencies advocate for organizational policy and practice changes to promote worker health and safety (CDC, 2015; NIOSH, 2014). In addition to national agencies, initiatives outlined by the US Affordable Care Act (US House of Representatives, 2010) support these recommendations. The Affordable Care Act (ACA) calls for amendment of the of Section 330 of the Public Health Service Act to establish wellness programs that include individualized wellness plans to address risk factors such as: overweight, smoking, excessive alcohol, lack of exercise, poor dietary habits, and high blood pressure. These individualized plans should include: nutritional counseling, exercise plans, alcohol or smoking cessation counseling, and stress management. In order to support these programs, the ACA employer-based wellness programs provisions under Part $U$ state that the Director of the Centers for Disease Control and Prevention will provide employers with consultation, assistance and tools to: measure participation in wellness programs; increase participation in these programs; develop measures to assess policy, environmental, and systems changes necessary to yield a positive impact; evaluate employee health changes, productivity, absenteeism, workplace injury, and medical costs. Part U states that the Director of the Centers for Disease Control and Prevention will also ensure capacity of workplace staff to implement and evaluate wellness programs and their impact. Both of these provisions support recommendations for worksites derived from this study.

The ACA also includes requirements for employee health plans. The provisions in the Part 1, Section 1302 outline requirements for essential health benefits. These include 
individual employee's access to services that include two key components that align with results from this study. Section 1302 states that health benefits should include access to mental health services and provide preventive and wellness services. Details regarding type of services or types of providers are not provided by the ACA provisions. Employees should be aware of which services are to be included in health plans. This will allow for an informed choice when selecting a health plan.

Results from this study indicate that workers at the computer technology company are at risk for illness despite access to an on-site health care provider. Employee health services offered at the computer company may be limited and not fully in compliance with ACA provisions. Based on the findings of the RAND Employer Survey (Matke, 2013), wellness programs are often offer limited services and rarely include organizational policy and practice changes. Worksite leadership should review current organizational culture, leadership practices, worksite wellness initiatives, and health insurance.

\section{Recommendations for Study Site}

This study found that a large portion of participants from a single computer technology company located in central Texas reported work stress. Job strain calculated through the ratio term yielded a $92 \%$ prevalence of job strain. Prevalence for job imbalance was $62 \%$. Approximately one-fifth (19\%) of the participants had prediabetes. Results also indicated that participants with prediabetes tended to have poor eating (99\%) and moderate insomnia (23\%). Based on these findings, recommendations for the central Texas computer company would include screening for: stress, dietary patterns, and sleep

habits during the annual biometric screening exam. Stress, including work stress, can be assessed through surveys or by adding biomarkers of stress (e.g., cortisol) to usual fasting 
blood sampling collection. Dietary patterns and sleep habits can be assessed through surveys completed before, during, or after biometric screening exams.

Stress, dietary, and sleep data collected during the biometric health exam could help the computer company health care medical providers tailor individual wellness goals in partnership with the employee. Aggregate data could be used to develop companywide wellness initiatives for disease management and lifestyle interventions. A Rand Wellness Program Study (Matke et al., 2013) reported that worksite wellness programs can reduce the employer's average healthcare costs by $\$ 30$ per member each month. Disease management programs offered the bulk of savings, but lifestyle management programs also reduced cost. The study reported that lifestyle management interventions aimed at healthy eating or increasing exercise reduced absenteeism and may improve productivity. Lifestyle management programs that target risk factors for chronic disease such as T2DM have been shown prevent or delay illness (DPP, 2002).

\section{SUMMARY}

This study provided data to answer the research questions and provided a unique contribution to prediabetes empirical evidence. The prevalence of prediabetes in the participant sample within this study was lower than expected despite some risk factors, e.g., work stress and sleep disturbance. The prevalence of prediabetes in the participant sample within this study was much lower compared to US rates (19\% vs. 37\%). Prevalence of prediabetes increases with age, especially over 65. The participants' younger age $(M=37.2)$ may account for the lower prevalence of prediabetes. Risk for prediabetes increases with larger body mass, especially in the obese BMI range. Participants tended to be in the normal BMI range (49\%). Few participants were in the obese BMI range $(21.95 \%)$. 
The prevalences of job strain $(92 \%)$ and job imbalance $(64 \%)$ within the participant sample in this study were higher than US prevalence rates (4\%). The participant sample largely consisted of younger, White male, white-collar workers in a computer technology industry. Typically, older age, race/ethnicity other than White, and lower socioeconomic status is associated with risk of prediabetes, suggesting that the lower prevalence of prediabetes within the participant sample in this study is consistent with empirical data.

High job strain and job imbalance have been associated with white-collar jobs, which is consistent with the results in this study. White-collar workers have been reported to have higher mental strain (Ndjaboué et al., 2014), and, in workers in a computer technology job, higher intentions to exit their job solely because of high job strain or stress (Dhar \& Dhar, 2010). It is possible that the study findings of lower prevalence of prediabetes and higher prevalences of job strain and job imbalance are related to the demographic characteristics of the participant sample in the present study. High reports of work stress may have also been related to reports of low rewards and low decisional latitude, elements of work conditions.

In the present study, job imbalance was inversely associated with prediabetes; this was a new and unexpected finding from this study. Job imbalance was also found to be a significant predictor of prediabetes; job imbalance reduces the risk of prediabetes. Previous studies have reported the direct association of job imbalance and prediabetes. As stated previously, it is possible that individual characteristics may at least partially explain the contradictory findings. Future studies should include the ERI model overcommitment scale and other measures of individual work characteristics to explain the current findings. 
Interestingly, this study found a significant relationship between work stress (job strain and job imbalance) and global and event-specific stress stress. This suggests that both measures of work stress included in this study (job strain and job imbalance) are captured within stress measures. This is a new finding that warrants further exploration in future studies.

Another interesting finding was that this study found that reports of sleep disturbance were associated with prediabetes and sleep disturbance was a significant predictor of prediabetes. Sleep disruption and poor sleep quality have been shown to be related to negative changes in general metabolism and, especially, glucose metabolism. The findings in this study suggest a more significant role of sleep disturbance and prediabetes. Sleep disturbance in this study predicted prediabetes. It is beyond the scope of this study to determine how sleep disturbance and prediabetes are physiologically related, however, future studies can further explore this physiological link.

The findings in this study provided a unique contribution to nursing science. A conceptual model, Conceptual Model for Metabolic Risk, included a comprehensive outline of the variables proposed to be associated with prediabetes. The results of this study that indicated a relationship between sociocultural, health behavior, and metabolic variables with prediabetes were congruent with previous studies, except for the relationship between job imbalance and prediabetes. Job imbalance was found to be inversely related to prediabetes and reduce the overall risk. Three significant predictors were identified in this study.

Current findings are limited to a single computer technology company in central Texas. Future studies should continue to examine the relationship between job imbalance and prediabetes in a larger, more diverse population. The relationship between sleep and job imbalance and sleep and prediabetes warrants further exploration. Future studies 
should also be duplicated in varying industries to determine if findings remain consistent with different worker populations. 


\title{
Appendices
}

\begin{abstract}
APPENDIX A
Hello,

Thank you for agreeing to participate in my dissertation research study. It means so much to me that you would choose to help. In order to participate in the study you must give your consent. Please read the information below. Follow the link to consent and begin the study. The surveys should take about 20 minutes.
\end{abstract}

Follow this link to the Survey:

$\$\{1: / /$ SurveyLink?d=Take the Survey $\}$

The password for the study is prediabetes.

Or copy and paste the URL below into your internet browser:

$\$\{1: / /$ SurveyURL $\}$

\section{Consent to Participate in Internet Research}

\section{Identification of Investigator and Purpose of Study}

You are invited to participate in a research study, entitled Prevalence and Characteristics of Prediabetes in Workers in a Technical Industry. The study is being conducted by Raquel Reynolds, doctoral student at the School of Nursing of The University of Texas at Austin, 1701 Red River Street, Austin, TX 78750, (512) 567-3075.

The purpose of this research study is to examine prediabetes in the workplace. Your participation in the study will contribute to a better understanding of factors that contribute to prediabetes. You are free to contact the investigator at the above address and phone number to discuss the study. You must be at least 25 years old to participate.

If you agree to participate:

- The online surveys will take approximately 20-30 minutes of your time.

- You will complete surveys about stress, coping, work stress, diet, exercise, and sleep one time only.

- You will not be compensated.

\section{Risks/Benefits/Confidentiality of Data}

There are no known risks. There will be no costs for participating, and there may be no direct benefit to you from participating. Your name and email address will be kept only during the data collection phase for tracking purposes only. A unique identification number will be used on your surveys. This number will be kept with your name and email address in a file separate from the data. Only my supervising professor and I will have access to the data during data collection. Identifying information will be stripped from the final dataset. 


\section{Participation or Withdrawal}

Your participation in this study is voluntary. You may decline to answer any question and you have the right to withdraw from participation at any time. Withdrawal will not affect your relationship with The University of Texas nor with your employer in anyway. If you do not want to participate either simply stop participating or close the browser window.

If you do not want to receive any more reminders, you may email me at rreynolds@utexas.edu or follow the link to opt out of future emails.

\section{Contacts}

If you have any questions about the study or need to update your email address contact the researcher, Raquel Reynolds, at (512) 567-3075 or send an email to rreynolds@utexas.edu. This study has been reviewed by The University of Texas at Austin Institutional Review Board and the study number is [2014-02-0011].

\section{Questions about your rights as a research participant.}

If you have questions about your rights or are dissatisfied at any time with any part of this study, you can contact, anonymously if you wish, the Institutional Review Board by phone at (512) 4718871 or email at orsc@uts.cc.utexas.edu.

If you agree to participate, follow this link to the Survey: $\$\{1: / /$ SurveyLink?d=Take the Survey $\}$

The password for the study is prediabetes.

Or copy and paste the URL below into your internet browser:

$\$\{1: / /$ SurveyURL $\}$

Follow the link to opt out of future emails: $\$\{1: / / O p t O u t L i n k ? d=C l i c k$ here to unsubscribe $\}$

Thank you,

Raquel Reynolds

\section{Please print a copy of this document for your records.}




\section{APPENDiX B}

\section{Demographic Information Sheet}

What is your age in years?

With what gender do you identify?

$\begin{array}{ll}\square & \text { Male } \\ \square & \text { Female } \\ \square & \text { Other }\end{array}$

With what race group(s) do you identify?

$\begin{array}{ll}\square & \text { White } \\ \square & \text { African American } \\ \square & \text { Asian } \\ \square & \text { Native American }\end{array}$

Do you belong to the Hispanic/Latino ethnic group?

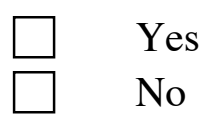

What is the highest level of education you have completed?

$\square \quad$ Primary / Grade school

$\square \quad$ High school

Trade / Technical school

College / University

Graduate school

What is your marital status?

\begin{tabular}{|ll}
$\square$ & Single / Never married \\
$\square \quad$ & Married \\
$\square \quad$ & Divorced \\
$\square \quad$ & Widowed
\end{tabular}


Have you ever had / been told that you have:

Gestational diabetes

High birth weight baby (female $>9$ lbs., male $>10$ lbs.)

Polycystic Ovarian Syndrome

None of the above

Do you have any relatives who have diabetes? How many?

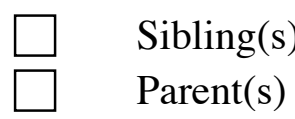

In what type of job do you currently work?

$\square \quad$ Management
$\square \quad$ Finance or Legal
$\square \quad$ Office and Administrative support
$\square \quad$ Engineering and Technical
$\square \quad$ Assembly and Fabrication
$\square \quad$ Operations and Production
$\square \quad$ Maintenance and Custodial
$\square \quad$ Other
If Other, please specify:

In what job setting do you work?

$\begin{array}{ll}\square \quad & \text { Private office } \\ \square \quad & \text { Cubicle or Open desk } \\ \square \quad & \text { Common or public area } \\ \square \quad & \text { Machine room or Workshop } \\ \square \quad & \text { Factory floor } \\ \square \quad & \text { Clean room } \\ \square \quad & \text { Other } \\ & \text { If Other, please specify: }\end{array}$


How many hours of the workday do you:

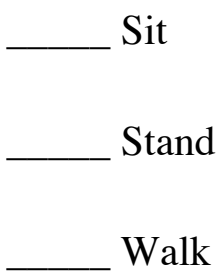

What hours do you normally work?

__ Hours per week

Start Time

Finish Time

Have you ever smoked?

$\square \quad$ No
$\square \quad$ Yes

If Yes, when did you stop?

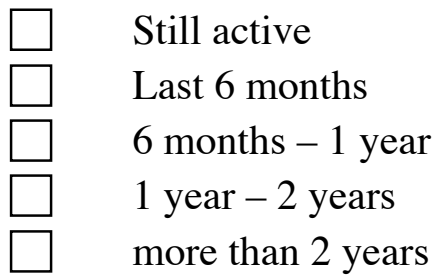

Do you drink alcoholic beverages?
How much did / do you smoke? cigarettes per day OR packs per day 
If Yes, how often do you drink?

1-2 times per month

1-2 times per week

3-4 times per week

5-6 times per week

daily
On each occasion, how much did you drink?

$\begin{array}{ll}\square \quad & 1-2 \text { drinks } \\ \square \quad & 3-4 \text { drinks } \\ \square \quad & 5-6 \text { drinks } \\ \square \quad & \text { more than 6 }\end{array}$




\section{Formulas for JCQ scale scores}

Job skill diucretion $=\left[q 1+q 3+q 5+q 7+q 9+5-q^{2}\right]+2$.

Rexrible rango

$12-48$

Job decinion-making autbority $=\left[2 *\left(q^{4}+q^{6}+q 8\right)\right]^{2}$.

$12-48$

Job demands $=3^{*}(\mathrm{q} 10+\mathrm{q} 11)+2+(15-\mathrm{q} 13-\mathrm{q} 14-\mathrm{q} 15)$.

$12-48+6+$

Job decivion latitude $=$ akill discretion + decision-maling authority .

$24-96$

We combine skill discretion ucale and decivion-making authority scale to create a new scale - Job dacision latitude (ninge 24-96). In addition job damands (nage 12-45) is calculatod from itama $10,11,13,14,15$ as abowe.

"Job strin" can be than be dofined in 1 of throe ways:

1) a score above the sample medien on job demands as woll as below the sample median on job dacision latitude. (NA)

2) a score abose the nationsl average on job damands as well as belor the national avarage on job dacision latitade.

To determine "job strain" it will be necessary to we population averages (see mext page).

Job strain is defined as above the average on demands, below average on decision latitude.

In addition, we can calculate,

3) A job struin ratio term: (Demands+2)/Decinion-Latituda.

A score $>1$ would indicate job strain.

4) calculate a r score for yourself and your mbjects to obtain a vanse of the magritude of job strain (see formula on next page)

Additional scale formulas for social support and job insecurity bolow:

Co-workar support $=q 17+q 18+q 19+q 20$.

Superviser support $=q 21+q 22+q 23+q 24$.

Job insecurity $=q 25+q 27+5-q 16$. 
National sverages for JCQ scales from 1969, 1972, and 1977 U.S. Quality of Employment Survers (4,495 men and women)

\begin{tabular}{|c|c|c|}
\hline & Averase & Devi \\
\hline Job skill divcretion $=$ & 33.5 & 8.50 \\
\hline Job decision-making autbority = & 36.8 & 990 \\
\hline Job decision latitude $=$ & 70.3 & 15.6 \\
\hline Job demands = & 30.9 & 8.48 \\
\hline Co-workar support $=$ & 12.73 & 2.53 \\
\hline Supervisor support $=$ & 11.94 & 4.85 \\
\hline Total socisl support $=$ & 24.6 & 4.26 \\
\hline Job insecurity = & 4.91 & 1.97 \\
\hline Decision latitade = & 70.20 & 15.87 \\
\hline Prychological job demands = & 30.26 & 7.17 \\
\hline
\end{tabular}

Sourcen: Kanuek RA, Gordon G, Pietrokovily C, Frose M, Pieper C, Schrartz J, Fry L, Schirar D.

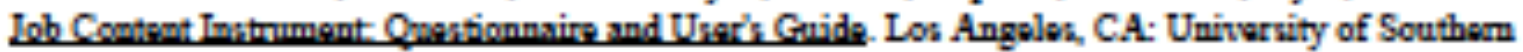
Califormin, 1985.

Kansak R, Brisuon C, Kawnlami N, Houtmen I, Bongars P, Amick B. The Job Coutant Qusationnaire (JCQ): An instrument for internationally comparative asessuments of prychosocial job charactaristicn. Lecoup Hesilth Parcholery 1998;3-322-355.

(Plus unprabliabed update, Robert Karziak, Novambar 2000)

Computation of $\mathbf{z}$-score: $\mathbf{z}=$ (mean of rour sample) - (national mesn) mational standard deviation 


\section{APPENDiX D}

\section{Insomnia Severity Index}

The Insomnia Severity Index has seven questions. The seven answers are added up to get a total score. When you have your total score, look at the 'Guidelines for Scoring/Interpretation' below to see where your sleep difficulty fits.

For each question, please CIRCLE the number that best describes your answer.

Please rate the CURRENT (i.e. LAST 2 WEEKS) SEVERITY of your insomnia problem(s)

\begin{tabular}{|l|c|c|c|c|c|}
\hline \multicolumn{1}{|c|}{ Insomnia Problem } & None & Mild & Moderate & Severe & Very Severe \\
\hline 1. Difficulty falling asleep & 0 & 1 & 2 & 3 & 4 \\
\hline 2. Difficulty staying asleep & 0 & 1 & 2 & 3 & 4 \\
\hline 3. Problems waking up too early & 0 & 1 & 2 & 3 & 4 \\
\hline
\end{tabular}

4. How SATISFIED/DISSATISFIED are you with your CURRENT sleep pattern?

Very Satisfied Satisfied Moderately Satisfied Dissatisfied Very Dissatisfied

$\begin{array}{llrrr}0 & 1 & 2 & 3 & 4\end{array}$

5. How NOTICEABLE to others do you think your sleep problem is in terms of impairing the quality of your life?

Not at all

Noticeable

0

A Little

1

Somewhat $\quad \underset{2}{3}$

Very Much Noticeable

4

6. How WORRIED/DISTRESSED are you about your current sleep problem?
Not at all
Worried
0
A Little
Somewhat
Much
Very Much Worried
2
3
4

7. To what ex tent do you consider your sleep problem to INTERFERE with your daily functioning (e.g. daytime fatigue, mood, ability to function at work/daily chores, concentration, memory, mood, etc.) CURRENTLY?

Not at all

Interfering

0
A Little
1
Somewhat
2 $\underset{3}{\text { Much }}$
Very Much Interfering

4

\section{Guidelines for Scoring/Interpretation:}

Add the scores for all seven items (questions $1+2+3+4+5+6+7$ ) $=$ your total score

Total score categories:

$0-7=$ No clinically significant insomnia

8-14 = Subthreshold insomnia

15-21 = Clinical insomnia (moderate severity)

$22-28$ = Clinical insomnia (severe) 


\section{Appendix E}

REAPS (Rapid Eating Assessment for Participants - Shortened Version)
CJSegal-Isaacson, EdD RD, Judy-Wylie-Rosett, EdD RD, Kim Gans, PhD, MPH

\begin{tabular}{|c|c|c|c|c|}
\hline In an average week, how often do you: & $\begin{array}{c}\text { Usuallyl } \\
\text { Often }\end{array}$ & Sometimes & $\begin{array}{l}\text { Rarelyl } \\
\text { Never }\end{array}$ & $\begin{array}{l}\text { Does not } \\
\text { apply to me }\end{array}$ \\
\hline 1. Skip breakfast? & O & o & o & \\
\hline 2. Eat 4 or more meals from sit-down or take out restaurants? & o & o & o & \\
\hline $\begin{array}{l}\text { 3. Eat less than } 2 \text { servings of whole grain products or high fiber } \\
\text { starches a day? Serving }=1 \text { slice of } 100 \% \text { whole grain bread; } \\
1 \text { cup whole grain cereal like Shredded Wheat, Wheaties, } \\
\text { Grape Nuts, high fiber cereals, oatmeal, } 3-4 \text { whole grain } \\
\text { crackers, } 1 / \text { c cup brown rice or whole wheat pasta, boiled or } \\
\text { baked potatoes, yuca, yams or plantain. }\end{array}$ & o & $\mathbf{O}$ & O & \\
\hline $\begin{array}{l}\text { 4. Eat less than } 2 \text { servings of fruit a day? } \\
\text { Serving = } 1 / 2 \text { cup or } 1 \text { med. fruit or } \% \text { cup } 100 \% \text { fruit juice. }\end{array}$ & $\mathrm{O}$ & $\mathbf{O}$ & $\mathrm{O}$ & \\
\hline $\begin{array}{l}\text { 5. Eat less than } 2 \text { servings of vegetables a day? } \\
\text { Serving }=1 / 2 \text { cup vegetables, or } 1 \text { cup leafy raw vegetables. }\end{array}$ & O & o & o & \\
\hline $\begin{array}{l}\text { 6. Eat or drink less than } 2 \text { servings of milk, yogurt, or cheese a } \\
\text { day? } \\
\text { Serving = } 1 \text { cup milk or yogurt; } 1 \%-2 \text { ounces cheese. }\end{array}$ & o & $\mathbf{0}$ & O & \\
\hline $\begin{array}{l}\text { 7. Eat more than } 8 \text { ounces (see sizes below) of meat, chicken, } \\
\text { turkey or fish per day? } \\
\text { Note: } 3 \text { ounces of meat or chicken is the size of a deck of cards or } \\
\text { ONE of the following: } 1 \text { regular hamburger, } 1 \text { chicken breast or leg } \\
\text { (thigh and drumstick), or } 1 \text { pork chop. }\end{array}$ & $\mathbf{0}$ & $\mathbf{0}$ & o & $\begin{array}{l}\text { Rarely eat meat, } \\
\text { chicken, turkey or } \\
\text { fish } \\
\text { O }\end{array}$ \\
\hline $\begin{array}{l}\text { 8. Use regular processed meats (like bologna, salami, corned } \\
\text { beef, hotdogs, sausage or bacon) instead of low fat processed } \\
\text { meats (like roast beef, turkey, lean ham; low-fat cold } \\
\text { cuts/hotdogs)? }\end{array}$ & $\mathbf{0}$ & $\mathbf{0}$ & O & $\begin{array}{c}\text { Rarely eat } \\
\text { processed meats } \\
\text { O }\end{array}$ \\
\hline $\begin{array}{l}\text { 9. Eat fried foods such as fried chicken, fried fish, French fries, } \\
\text { fried plantains, tostones or fried yuca? }\end{array}$ & O & $\mathbf{0}$ & o & \\
\hline $\begin{array}{l}\text { 10. Eat regular potato chips, nacho chips, com chips, crackers, } \\
\text { regular popcorn, nuts instead of pretzels, low-fat chips or low- } \\
\text { fat crackers, air-popped popcom? }\end{array}$ & O & O & O & $\begin{array}{l}\text { Rarely eat these } \\
\text { snack foods } \\
\text { O }\end{array}$ \\
\hline $\begin{array}{l}\text { 11. Add butter, margarine or oil to bread, potatoes, rice or } \\
\text { vegetables at the table? }\end{array}$ & O & o & $\mathbf{0}$ & \\
\hline $\begin{array}{l}\text { 12. Eat sweets like cake, cookies, pastries, donuts, muffins, } \\
\text { chocolate and candies more than } 2 \text { times per day. }\end{array}$ & 0 & $\mathbf{0}$ & $\mathrm{O}$ & \\
\hline $\begin{array}{l}\text { 13. Drink } 16 \text { ounces or more of non-diet soda, fruit drink/punch or } \\
\text { Kool-Aid a day? } \\
\text { Note: } 1 \text { can of soda }=12 \text { ounces }\end{array}$ & O & O & $\mathbf{O}$ & \\
\hline & \multicolumn{3}{|c|}{ YES } & NO \\
\hline $\begin{array}{l}\text { 14. You or a member of your family usually shops and cooks rather } \\
\text { than eating sit-down or take-out restaurant food? }\end{array}$ & \multicolumn{3}{|c|}{ o } & O \\
\hline 15. Usually feel well enough to shop or cook. & \multicolumn{3}{|c|}{ O } & 0 \\
\hline $\begin{array}{l}\text { 16. How willing are you to make changes in your eating habits in } \\
\text { order to be healthier? }\end{array}$ & $\begin{array}{c}1 \\
\text { Vory } \\
\text { willing }\end{array}$ & 2 & 3 & $\begin{array}{c}6 \\
\text { Not at all } \\
\text { willing }\end{array}$ \\
\hline
\end{tabular}




\section{APPENDiX F}

\section{Godin Leisure-Time Exercise Questionnaire}

Considering a 7-Day period (a week), how many times on the average do you do the following kinds of exercise for more than $\mathbf{1 5}$ minutes during your free time (write on each line the appropriate number).

Times Per

Week

\section{a) STRENUOUS EXERCISE \\ (HEART BEATS RAPIDLY)}

(i.e. running, jogging, hockey, football, soccer,

squash, basketball, cross country skiing, judo,

roller skating, vigorous swimming,

vigorous long distance bicycling)

b) MODERATE EXERCISE

(NOT EXHAUSTING)

(i.e. fast walking, baseball, tennis, easy bicycling,

volleyball, badminton, easy swimming, alpine skiing,

popular and folk dancing)

\section{c) MILD EXERCISE}

\section{(MINIMAL EFFOR'T)}

(i.e. yoga, archery, fishing from river band, bowling, horseshoes, golf, snow-mobiling, easy walking)

2. Considering a 7-Day period (a week), during your leisure-time, how often do you engage in any regular activity long enough to work up a sweat (heart beats rapidly)?
OFTEN
SOMETIMES
NEVER/RARELY
1.
2
3. 


\section{APPENDix G}

\section{Effort Reward Imbalance Questionnaire}

\begin{tabular}{|c|}
\hline $\begin{array}{l}\text { Strongly } \\
\text { disagree }\end{array}$ \\
\hline
\end{tabular}

(1)

(2)

(3)

ERI1 I have constant time pressure due to a heavy work load.

ERI2 I have many interruptions and disturbances while performing my job.

ERI3 I have a lot of responsibility in my job.

ERI4 I am often pressured to work overtime.

ERI5 My job is physically demanding.

ERI6 Over the past few years, my job has become more and more demanding.

ERI7 I receive the respect I deserve from my superior or a respective relevant person.

ERI8 I experience adequate support in difficult situations.

ERI9 I am treated unfairly at work. Reverse coding

ERI10 My job promotion prospects are poor. Reverse coding

ERI11 I have experienced or I expect to experience an undesirable change in my work situation. Reverse coding

ERI12 My employment security is poor. Reverse coding

ERI13 My current occupational position adequately reflects my education and training.

ERI14 Considering all my efforts and achievements, I receive the respect and prestige I deserve at work.

ERI15 Considering all my efforts and achievements, my job promotion prospects are adequate.

ERI16 Considering all my efforts and achievements, my salary / income is adequate. 


\section{APPENDIX H}

Bricf ApproachlAvoidance Coping Questionnaine (BACQ)

How do you usually cope with problems and illness?

The questions on this page deal with how you usuglly act in relation to problems and disegse. For each itern, place a tick in the box that fits best with what you think about youndf just now. The questions gre written in $\mathrm{T}$ form, and you place your tick depending on how much you agree/disagree. The purpose of the questicas is to make you think gbout whether or not you gre satisfied with the way you regct to problems gnd illness.

Response categaries:

Agree completely 5

Tend to agree 4

Yes and no 3

Tend to disagree 2

Disagree completely $\quad 1$

Itews

1. I say so if I gm angy or sad.

2. I like to talk with a few chosen pecple when things get too much for me

3. I make gn active effort to find a solution to my problems.

4. Physical exercise is impartant to me.

5. I think something positive could come out of my complaints/problems.

6. I fimly believe that my problems will decrease (and my situation improve).

7. I try to farget my problems.

8. I put my problems behind me by concentrating on something else.

9. I bury myself in work to keep my problems gt a distance.

10. I often find it difficult to do something new.

11. I am well on the way towards feeling I have given up.

12. I withdrgw from other peaple when things get difficult. 


\section{APPENDIX I}

\section{PSS-14}

\section{INSTRUCTIONS:}

The questions in this scale ask you about your feelings and thoughts during THE LAST MONTH. In each case, you will be asked to indicate your response by placing an " $\mathrm{X}$ " over the circle representing HOW OFTEN you felt or thought a certain way. Although some of the questions are similar, there are differences between them and you should treat each one as a separate question. The best approach is to answer fairly quickly. That is, don't try to count up the number of times you felt a particular way, but rather indicate the alternative that seems like a reasonable estimate.

\begin{tabular}{ccccc} 
Never & $\begin{array}{c}\text { Almost } \\
\text { Never }\end{array}$ & Sometimes & $\begin{array}{c}\text { Fairly } \\
\text { Often }\end{array}$ & $\begin{array}{c}\text { Very } \\
\text { Often }\end{array}$ \\
0 & 1 & 2 & 3 & 4 \\
\hline
\end{tabular}

1. In the last month, how often have you been upset because of something that happened unexpectedly?

2. In the last month, how often have you felt that you were unable to control the important things in your life?

3. In the last month, how often have you felt nervous and "stressed"?

4. In the last month, how often have you dealt successfully with day to day problems and annoyances?

5. In the last month, how often have you felt that you were effectively coping with important changes that were occurring in your life?

6. In the last month, how often have you felt confident about your ability to handle your personal problems?

7. In the last month, how often have you felt that things were going your way?

8. In the last month, how often have you found that you could not cope with all the things that you had to do?

9. In the last month, how often have you been able to control irritations in your life?

10. In the last month, how often have you felt that you were on top of things?

version: $11 / 26 / 2014$ 


\begin{tabular}{ccccc} 
Never & $\begin{array}{c}\text { Almost } \\
\text { Never }\end{array}$ & Sometimes & $\begin{array}{c}\text { Fairly } \\
\text { Often }\end{array}$ & $\begin{array}{c}\text { Very } \\
\text { Often }\end{array}$ \\
0 & 1 & 2 & 3 & 4 \\
\hline
\end{tabular}

11. In the last month, how often have you been angered because of things that happened that were outside of your control?

12. In the last month, how often have you found yourself thinking about things that you have to accomplish?

13. In the last month, how often have you been able to control the way you spend your time?

14. In the last month, how often have you felt difficulties were piling up so high that you could not overcome them? 


\section{References}

Abdul-Ghani, M., \& DeFronzo, R.A. (2009). Pathophysiology of prediabetes. Current Diabetes Reports, 9(3), 193-199.

Adams, O.P. (2013). The impact of brief high-intensity exercise on blood glucose levels. Diabetes, Metabolic Syndrome and Obesity: Targets and Therapy, 6, 113-122.

Akerstedt, T., Nordin, M., Alfredsson, L., Westerholm, L., \& Kecklund, G. Predicting changes in sleep complaints from baseline values and changes in work demands, work control, and work preoccupation - The WOLF project. Sleep Medicine, 13(1), 73-80.

Akinci, F., Healey, B.J., \& Coyne, J.S. (2003). Improving the health status of US working adults with Type 2 Diabetes Mellitus. Disease Management and Health Outcomes, 11(8), 489-498.

Albertsen, K., Rafnsdottir, L., Grimsmo, A., Tomasson, K., \& Kauppinen, K. (2008). Workhours and worklike balance. Scandnavian Journal of Work, Environment and Health, Supplement, (5), 14-21.

Al-Daghri, N.M., Al-Attas, O.S., Al-Rubean, K., Mohieldin, M., Al-Katari, M., Jones, A.F., \& Kumar, S. (2007). Serum leptin and its relation to anthropometric measures of obesity in pre-diabetic Saudis. Cardiovascular Diabetology, 6(18). doi: $10.1186 / 1475-2840-6-18$

American Association for Clinical Chemistry. (2013). HDL Cholesterol. Retrieved from, http://labtestsonline.org/understangin/analytes/hdl/tab/test

American Diabetes Association. (1997). Report of the Expert Committee on the Diagnosis and Classification of Diabetes Mellitus. The Expert Committee on the Diagnosis and Classification of Diabetes Mellitus. Diabetes Care, 20(7), 11831197.

American Diabetes Association. (2003). Follow-up report on the diagnosis of diabetes mellitus: the Expert Committee on the Diagnosis and Classification of Diabetes Mellitus. Diabetes Care, 26(11), 3160-3167.

American Diabetes Association. (2010). Diagnosis and classification of diabetes mellitus: Position statement. American Diabetes Association. Diabetes Care, 33(Supp 1), 562-569.

American Diabetes Association. (2013). Diabetes basis: All about cholesterol. Retrieved from, $\quad$ http://www.diabetes.org/diabetes-basics/prevention/checkupamerica/cholesterol.html

American Diabetes Association. (2013a). Diabetes basis: Prediabetes. Retrieved from, http://www.diabetes.org/diabetes-basics/prevention/pre-diabetes/ 
American Diabetes Association. (2013b). Diabetes basics: Common terms. Retrieved from, http://www.diabetes.org/diabetes-basics/common-terms/common-terms-fk.html

American Diabetes Association. (2013c). Diabetes basics: Diabetes statistics. Retrieved from, http://www.diabetes.org/diabetes-basics/diabetes-statistics/

American Diabetes Association. (2013d). Diabetes basics: Age, race, gender, \& family history. Retrieved from, http://www.diabetes.org/diabetesbasics/prevention/checkup-america/nonmodifiables.html

American Heart Association. (2012a). About high blood pressure. Retrieved from, http://www.heart.org/HEARTORG/Conditions/HighBloodPressure/AboutHighBl oodPressure/About-High-Blood-Pressure_UCM_002050_Article.jsp

American Heart Association. (2012b). Understand your risk for diabetes. Retrieved from, http://www.heart.org/HEARTORG/Conditions/Diabetes/UnderstandYourRiskfor Diabetes/Understand-Your-Risk-for-Diabetes UCM 002034 Article.jsp

American Heart Association. (2013c). Triglycerides. Retrieved from, http://www.heart.org/HEARTORG/GettingHealthy/NutritionCenter/Triglycerides UCM 306029 Article.jsp

American Psychological Association. (2014) Practice guidelines for LGB clients. Retrieved from, http://www.apa.org/pi/lgbt/resources/guidelines.aspx

American Psychological Association. (2013). Work and Well-Being Survey: American Psychological Association. Harris Interactive. American Psychological Association Center for Organizational Excellence. Retrieved from, http://www.apaexcellence.org/assets/general/2013-work-and-wellbeing-surveyresults.pdf

American Psychological Association. (2012). Stress in America: Our health at Risk. Retrieved from, https://www.apa.org/news/press/releases/stress/2011/final2011.pdf

American Psychological Association. (2014). Stress effects on the body. Retrieved from, http://www.apa.org/helpcenter/stress-body.aspx

Antuna-Puente, B., Feve, B., Fellahi, S., \& Bastard, J.P. (2008). Adipokines: The missing link between insulin resistance and obesity. Diabetes and Metabolism, 34(1), 211.

Astvik, W., \& Melen, M. (2012). Coping with the imbalance of job demands and resources: A study of different coping patterns and implications for health and quality in human service work. Journal of Social Work, 13(4), 337-360.

Baik, I., \& Shin, C. (2008). Prospective study of alcohol consumption and metabolic syndrome. American Journal of Clinical Nutrition, 87(5), 1455-1463. 
Baker, D.P., Leon, J., Smith Greenway, E.G., Collins, J., \& Movit, M. (2011). The education effect on population health: A reassessment. Population and Development Review, 37(2), 307-332.

Banerjee, M. \& Cruikshank, J.K. (2004). 'Prediabetes': Is the term useful? British Journal of Diabetes and Vascular Disease, 4(4), Retrieved from, http://www.medscape.com/viewarticle/489584

Bang, H., Edwards, A.M., Bomback, A.S., Ballantyne, C.M., Brillon, D., Callahan, M.A....Kern, L.M. (2009). Development and validation of a patient selfassessment score for diabetes risk. Annals of Internal Medicine, 151(11), 775-783.

Bardini, G., Kicembrini, I., Cresci, B., \& Rotella, C.M. (2010). Inflammation markers and metabolic characteristics of subjects with 1-h plasma glucose levels. Diabetes Care, 33(2), 411-413.

Barron, R.M., \& Kenny, D.A. (1986). The moderator-mediator variable distinction in social psychological research: Conceptual, strategic, and statistical considerations. Journal of Personality and Social Psychology, 51(6), 1173-1182.

Bastien, C.H., Villieres, A., \& Morin, C.M. (2001). Validation of the Insomnia Severity Index as an outcome measure for insomnia research. Sleep Medicine, 2(4), 297307.

Bazzano, L.A., Serdula, M., \& Lin, S. (2005). Prevention of Type 2 Diabetes by diet and lifestyle modification. Journal of the American College of Nutrition, 24(5), 310319.

Bergman, B.C., Perreault, L., Hunerdosse, D., Kerege, A., Playdon, M., Samek, A.M., \& Eckel, R.H. (2012). Novel and reversible mechanisms of smoking-induced insulin resistance in humans. Diabetes, 61(12), 3158-3166.

Biggi, N., Consonni, K., Galluzzo, V., Sogliani, M., \& Costa, G. (2008). Metabolic syndrome in permanent night workers. Chronobiology International, 25(2\&3), 443-454.

Black, P.H. (2003). The inflammatory response is an integral part of the stress response: Implications for atherosclerosis, insulin resistance, type II diabetes and metabolic syndrome. Brain, Behavior and Immunity, 17(5), 350-364.

Bloomgarden, Z.T. (2008a). American College of Endocrinology Pre-diabetes Consensus Conference: Part one. Diabetes Care, 31(10), 2062-2068.

Bloomgarden, Z.T. (2008b). American College of Endocrinology Pre-diabetes Consensus Conference: Part two. Diabetes Care, 31(11), 2222-2228.

Bloomgarden, Z.T. (2008c). American College of Endocrinology Pre-diabetes Consensus Conference: Part three. Diabetes Care, 31(12), 2404-2409. 
Brekke, H.K., Lenner, R.A., Taskinen, M-R., Mansson, J-E., Funahashi, T., Matsuzawa, Y., \& Jansson, P-A. (2004). Lifestyle modification improves risk factors in type 2 diabetes relatives. Diabetes Research and Clincial Practice, 68(1), 18-28.

Brunello, G., Fort, M., Schneeweis, N., \& Winter-Ebmer, R. (2011). The causal effect of education on health: What is the role of health behaviors? Discussion Paper. Institute for the Study of Labor, Bonn, Germany. Retrieved from, http://ftp.iza.org/dp5944.pdf

Burnner, E.J., Chandola, T., \& Marmot, M.G. (2007). Prospective effect of job strain on general and central obesity in the Whitehall II Study. American Journal of Epidemiology, 165(7), 828-837.

Buiso, T.J., Butterworth, S., \& Linden, A. (2007). A conceptual framework for targeting prediabetes with lifestyle, clinical, and behavioral management interventions. Disease Management, 10(1), 6-14.

Buxton, O.M., Pavlova, M., Reid, E.W., Wang, W., Simonson, D.C., \& Adler, G.K. (2010). Sleep restriction for 1 week reduces insulin sensitivity in health men. Diabetes, 59(9), 2126-2133.

Byberg, S., Hansen, A.L.S., Christensen, D.L., Vistisen, D. Aadahl., M., Linneberg, A., \& Witte, D.R. (2012). Sleep duration and sleep quality are associated differently with alterations of glucose homeostasis. Diabetic Medicine, 29(9), e3540360.

Byrne, B. (2006). Structural equation modeling with EQS: Basic concepts, applications, and programming. [ $\left.2^{\text {nd }} E d.\right]$ Lawrence Erlbaum Associates: Mahwah, NJ.

Byrne, B. (2012). Structural equation modeling with Mplus: Basic concepts, applications, and programming. Taylor and Francis Group: New York, NY.

Carnethon, M., Whitsel, L.P., Franklin, B.A., Kris-Etherton, P., Milani, R., Pratt, C.A., \& Wagner, G.R. (2009). Worksite wellness programs for cardiovascular disease prevention: A policy statement from the American Heart Association. Circulation, 120(17), 1725-1741.

Cawley, J., Rizzo, J.A., \& Haas, K. (2008). The association of diabetes with job absenteeism costs among obese and morbidly obese workers. Journal of Occupational and Environmental Medicine, 50(5),

Centers for Disease Control and Prevention. (1999). National Occupational Research Agenda. Retrieved from, http://www.cdc.gov/niosh/docs/99-130/

Centers for Disease Control and Prevention. (2008). National diabetes fact sheet: General information and national estimates on diabetes in the United States, 2007. Atlanta, GA: U.S. Department of Health and Human Services.

Centers for Disease Control and Prevention. (2011a). 2011 National Diabetes Fact Sheet. Estimates of prediabetes prevalence using various definition criteria. Atlanta, GA: 
U.S. Department of Health and Human Services, Centers for Disease Control and Prevention, 2011.

Centers for Disease Control and Prevention. (2011b). Healthy weight - Its not a diet, it's a lifestyle! About BMI for adults. Retrieved from, http://www.cdc.gov/healthyweight/assessing/bmi/adult_bmi/

Centers Disease Control and Prevention. (2014). Health effects of cigarette smoking. Retrieved from, http://www.cdc.gov/tobacco/data_statistics/fact_sheets/health_effects/effects_cig smoking/

Centers Disease Control and Prevention. (2014). Alcohol use and health. Retrieved from, http://www.cdc.gov/alcohol/fact-sheets/alcohol-use.htm

Centers for Disease Control and Prevention. (2014). National healthy worksite program toolkit. $\quad$ Retrieved http://www.cdc.gov/nationalhealthyworksite/join/toolkit.html

Centers for Disease Control and Prevention. (2016). Workplace health promotion. Retrieved from, http://www.cdc.gov/workplacehealthpromotion/

Chandola, T., Brunner, E., \& Marmot, M. (2006). Chronic stress at work and the metabolic syndrome: Prospective study. British Medical Journal, (7540)332, 521. doi: $10.1136 / \mathrm{bmj} .38693 .435301 .80$

Chaput, J-P., Despres, J-P., Bouchard, C., Astrup, A., \& Tremblay, A. (2009). Sleep duration as a risk factor for the development of type 2 diabetes or impaired glucose tolerance: Analyses of the Quebec Family Study. Sleep Medicine, 10(8), 919-924.

Chui, K.C., Lee, N.P., Cohan, P., \& Chuang, L-M. (2000). Beta cell function declines with age in glucose tolerant Caucasians. Clinical Endocrinology, 53(5), 569-575.

Cohen, S., Kamarck, T., \& Mermelstein, R. (1983). A global measure of perceived stress. Journal of health and social behavior, 24(4), 385-396.

Cohen, S., \& Williamson, G.M. (1988). Perceived stress in a probability sample of the United States. The social psychology of health. Sage: Newbury Park.

Colberg, S.R., Sigal, R., Fernhall, B., Regensteiner, J.G., Blissmer, B.J., Rubin, R.R.,...Braun, B. (2010). Exercise and type 2 diabetes: The American College of Sports Medicine and the American Diabetes Association: Joint position statement. Diabetes Care, 33(12), 3147-e167.

Crispim, C.A., Waterhouse, J., Damso, A.R., Zimberg, I.Z., Padilha, H.G., Oyama, L.M., Tufik, S., \& de Mello, M.T. (2011). Hormonal appetite control is altered by shift work: A preliminary study. Metabolism, 60(12), 1726-1734. 
Cullmann, M., Hilding, A., Östenson, C.-G. (2011). Alcohol consumption and risk of prediabetes and type 2 diabetes development in a Swedish population. Diabetic Medicine,29(4), 441-452.

Cutler, D.M., \& Lleras-Muney, A. (2007). Education and health. Policy Brief \#9. University of Michigan, National Poverty Center. Retrieved from, http://www.npc.umich.edu/publications/policy_briefs/brief9/

Dagogo-Jack, S., Edeoga, C., Ebenibo, S., Nyenwe, E., \& Wan, J. (2014). Lack of racial disparitiy in incident prediabetes and glycemic progression among Black and White offspring of parents with Type 2 Diabetes: The Pathobiology of prediabetes in a Biracial Cohort (POP-ABC) study. Journal of Clinical Endocrinology and Metabolism, 99(6), E1078-E1087.

Daley, M., Morin, C.M., LeBlanc, M., Gregoire, J-P, \& Savard, J. (2009). The economic burden of insomnia: Direct and indirect costs for individuals with insomnia syndrome, insomnia symptoms, and good sleepers. Sleep,32(1), 55-65.

Davila, E.P., Florez, H., Fleming, L.E., Lee, D.J., Goodman, E., LeBlanc, W.G.,..Clarke, T. (2010). Prevalence of metabolic syndrome among U.S. Workers. Diabetes Care, 33(11), 2390-2395.

deAraújo, T.M., \& Karasek, R. (2008). Validity and reliability of the job content questionnaire in formal and informal jobs in Brazil. Scandinavian Journal of Work, Environment, \& Health, Supplement(6), 52-59.

deBacquer, D., Pelfrene, E., Clays, E., Mak, R., Moreau, M., deSmet, P., ...deBacker, G. (2005). Perceived job stress and incidence of coronary events: 3-year follow-up of the Belgian Job Stress Project cohort. American Journal of Epidemiology, 161(5), 434-441.

deBacquer, D, VanRisseghem, M., Clays, E., Kittel, F., DeBacker, G., \& Braeckman, L. (2009). Rotating shift work and the metabolic syndrome: A prospective study. International Journal of Epidemiology, 28(30, 848-854.

Deedwania, P.C. \& Fonseca, V.A. (2005). Diabetes, prediabetes, and cardiovascular risk: Shifting the paradigm. The American Journal of Medicine, 118(9), 941-945.

Dhar, R.L., \& Dhar, M. (2010). Job stress, coping process and intentions to leave: A study of information technology professionals working in India. The Social Science Journal, 47(3), 560-577. doi:10.1016/j.soscij.2010.01.006

deJonge, J. Bosma, H., Peter, R. \& Siegrist, J. (2000). Job strain, effort-reward imbalance and employee well-being: A large-scale cross-sectional study. Social Science and Medicine, 50(9), 1317-1327.

deLange, A.H., Kompier, M.A.J., Taris, T.W., Geurts, S.A.E., Beckers, D.G.J., Houtman, I.D., \& Bongers, P.M. (2009). A hard day's night: A longitudinal study on the 
relationships among job demands and job control, sleep quality and fatigue. Journal of Sleep Research, 1893), 374-383.

The Diabetes Prevention Program Research Group. (2002). The diabetes prevention program: Description of lifestyle intervention. Diabetes Care, 25(12), 2165-2171.

Djousse, L., Biggs, M.L., Mukamal, K.J., \& Siscovick, D.S. (2007). Alcohol consumption and type 2 diabetes among older adults: The Cardiovascular Health Study. Obesity, 15(7), 17-58-1765.

Donahue, R.P., Rejman, K., Rafalson, L.B., Dmochowski, J., Stranges, S., \& Trevisan, M. (2007). Sex differences in endothelial function markers before conversion to pre-diabetes: Does the clock start ticking earlier among women? The Western New York Study. Diabetes care, 30(2), 354-359.

Dullart, R.P.F., deVries, R., vanTol, A., \& Slulter, W.J. (2007). Lower plasma adiponectin is a marker of increased intima-media thickness associated with type 2 diabetes mellitus and with male gender. European Journal of Endocrinology, 156(3), 387-394.

Eriksson, J.G., Groop, L.,, Isomaa, B.,, Pyykkonen, A-J, Raikkonen, K., \& Tuomi, T. (2010). Stressful life events and the metabolic syndrome: The Prevalence, Prediction, and Prevention of Diabetes Study (PPP) - Botina Study. Diabetes Care, 33(2), 378-384.

Esposito, K., Nappo, F., Marfella, R., Giugliano, G., Giugliano, F., Ciotola, M., ...., \& Giugliano, D. (2002). Inflammatory cytokine concentrations are acutely increased by hyperglycemia in humans: Role of oxidative stress. Circulation, 106(16), 2067-2072. doi: 10.1161/01.CIR.0000034509.14906.AE

Fairchild, A.J., \& MacKinnon, D.P. (2009). A general model for testing mediation and moderation effects. Prevention Science, 10(2), 87-99.

Fan, L., Blumenthal, J.A., Hinderliter, A.L., \& Sherwood, A. (2012). The effect of job strain on nighttime blood pressure dipping among men and women with high blood pressure. Scandnavian Journal of Work, Environment and Health - online first. doi: 10.5271/sjweh.3294

Faul, F., Erdfelder, E., Lang, A-G., \& Buchner, A. (2007). G*Power 3: A flexible statistical power analysis program for the social, behavioral, and biomedical sciences. Behavior Research Methods, 39(2), 175-191.

Feinstein, L., Sabates, R., Anderson, T.M., Sorhaindo, A., \& Hammond. C. (2006). What are the effects of education on health? Proceedings from the Copenhagen Symposium. Retrieved from, https://www1.oecd.org/edu/innovationeducation/37425753.pdf 
Finset, A., Steine, S., Haugli, L., Steen, E., \& Laeurm, E. (2002). The Brief Approach/Avoidance Coping Questionnaire: Development and validation. Psychology, Health, and Medicine, 7(1), 75-85.

Fonseca, R., \& Zheng, Y. (2011). The effect of education on health: Cross country evidence. Working Paper: Rand Labor and Population. Retrieved from, http://www.rand.org/content/dam/rand/pubs/working_papers/2011/RAND_WR86 $\underline{4 . p d f}$

Fransson, E.I.M., Alfredsson, L.S., deFaire, U.H., Knutsson, A., \& Westerholm, P.J.M. (2003). Leisure time, occupational and household physical activity, and risk factors for cardiovascular disease in working men and women: The WOLF study. Scandinavian Journal of Public Health, 31(5), 324-333.

Fransson, E.L., Heikkila, K., Nyberg, S.T., Zins, M., Westerlund, H., Westerholm, P., ...Kivimaki, M. (2012). Job strain as a risk factor for leisure-time physical inactivity: An individual-participant meta-analysis of up to 170,000 men and women. American Journal of Epidemiology, 176(12), 1078-1089.

Freak-Polli, R., Wolfe, R., \& Peeters, A. (2010). Risk of cardiovascular disease and diabetes in a working population with sedentary occupations. Journal of Occupational and Environmental Medicine, 52(11), 1132-1137.

Fujino, Y., Iso, H, Tamakoshi, A., Inaba, Y., Kolzumi, A., Kubo, T., ...the Japanese Collaborative Cohort Study Group. (2006). A prospective cohort study of shift work and risk of ischemic heart disease in Japanese male workers. American Journal of Epidemiology, 154(2), 128-135.

Fukunishi, I., Akimoto, M., Horikawa, N., Shirasaka, K., \& Yamazaki, T. (1998). Stress, coping, an social support in glucose tolerance abnormality. Journal of Psychosomatic Research, 45(4), 361-369.

Gadinger, M.C., Fischer, J.E >, Schneider, S., Fischer, G.C., Frank, G., \& Kromm, W. (2009). Female executives are particularly prone to sleep-disturbing effect of isolated high-strain jobs: A cross-sectional study in German-speaking executives. Journal of Sleep Research, 18(2), 229-237. doi: 10.1111/j.13652869.2008.00715.x

Gangwisch, J.E., Heymsfield, S.B., Boden-Albala, B., Bujis, R.M., Kreier, F., Pickering, T.G., ...Malaspina, D. (2007). Sleep duration as a risk factor for diabetes incidence in a large US sample, Sleep, 30(12), 1667-1673.

Gans, K.M., Riskica, P.M., Wylie-Rosett, J., Ross, E.M., Strolla, L.O., McMurray, J., \& Eaton, C.B. (2006). Development and evaluation of the nutrition component of the Rapid Eating and Activity Assessment for Patients (REAP): A new tool for primary care providers. Journal of Nutrition, Education and Behavior, 38(5), 286292. 
Gans, K.M., Ross, E., Barner, C.WQ., Wylie-Rosett, J., McMurray, J., \& Eaton, C. (2003). REAP and WAVE: New tools to rapidly assess/discuss nutrition with patients. The Journal of Nutrition133(2), 556S-62S.

Garber, A.J., Handelsman, Y., Einhorn, D., Bergman, D.., Bloomgarden, Z.T., Fonseca, V., ...Nesto, R.W. (2008). Diagnosis and management of prediabetes in the continuum of hyperglycema-When do the risks of diabetes begin? A consensus statement from the American College of Endocrinology and the American Association of Clinical Endocrinologists. Endocrine Practice, 14(7), 933-946.

Gavrila, A., Peng, C.K., Chan, J., Mietus, J.E., Goldberger, A.L., \& Mantzoros, C.S. (1998). Diurnal and ultradian dynamics of serum adiponectin in health men: Comparison with leptin, circulating soluble leptin receptor, and cortisol patterns. The Journal of Clinical Endocrinology and Metabolism, 88(6), 2838-2843. doi: 10.1210/jc.2002-021721

Gebel, E. (2011). The role of sleep in Type 2 diabetes. Diabetes Forcast: The Healthy Living Magazine, 64(5). Retrieved from, American Diabetes Association, http://forecast.diabetes.org/magazine/features/the-role-sleep-type-2-diabetes

Geiss, L.S., James, C., Gregg, E.W., Albright, A., Williamson, D.F.,\& Cowie, C. (2010). Diabetes risk reduction behaviors among U.S. adults with prediabetes. American Journal of Preventative Medicine, 38(4), 403-409.

Godin, G., \& Shepard, R.J. (1985). A simple method to assess exercise behavior in the community. Official Journal of the American College of Sports Medicine, 29(6), S36-S37.

Goldberg, R.B. (2009). Cytokine and cytokine-like inflammation markers, endothelial dysfunction, and imbalanced coagulation in development of diabetes and its complications. Journal of Clinical Endocrinology and Metabolism, 94(9), 31713182.

Grundy, S.M., Neeland, I.J., Turer, A.T., \& Vega, G.L. (2014). Ethnic and gender susceptibility to metabolic risk. Metabolic Syndrome and Related Disorders, 12(2), 110-116.

Gupta, A.K., Cornelissen, G., Pan, W., \& Halberg, F. (2010). Prediabetes is associated with abnormal circadian blood pressure variability. Journal of Human Hypertension, 22(9), 627-633.

Gunzler, D., Chen, T., Wu, P., \& Zhang, H. (2013). Introduction to mediation analysis with structural equation modeling. Shanghai Archives of Psychiatry, 25(6), 390394.

Hackworth, N., Moore, S.M., Hardie, E., Critchley, C., Buzwell, S., Crafti, N., \& Kyrios, M. (2007). A risk factor profile for pre-diabetes: Biochemical, behavioral, psychosocial, and cultural factors. Journal of Applied Psychology, 3(2), 14-26. 
Haffner, S. (2000). Obesity and the metabolic syndrome: The San Antonio Heart Study. British Journal of Nutrition, 83(Supp1), S67-S70.

Hafffner, S. (2003). Pre-diabetes, insulin resistance, and inflammation and CVD risk. Diabetes Research and Clinical Practice, 61(Supp1), S9-S18.

Haffner, S.M., Mykkanen, L., Festa, A., Burke, J.P., Stern, M.P. (1999). Insulin-resistant prediabetic subjects have more atherogenic risk factors than insulin-sensitive prediabetic subjects: Implications for preventing coronary heart disease during the prediabetic state. Circulation, 7(10), 975-980.

Hansen, A.M., Pearson, R., Garde, A.H., Karlson, B., \& Orbeck, P. (2006). Diurnal profiles of salivary cortisol on workdays among construction workers versus white collar workers. Scandnavian Journal of Work, Environment and Health, 2(Supp), 22-26.

Healthy People 2020. (2012). Occupational safety and health. Retrieved from, http://www.healthypeople.gov/2020/topicsobjectives2020/objectiveslist.aspx?topi cId $=30$

Healy, G.N., Dunstan, D.W., Salmon, J., Cerin, E., Shaw, J.E., Zimmet, P.Z., \& Owen, N. (2007). Objectively measured light-intensity physical activity is independently associated with 2-h plasma glucose. Diabetes Care, 30(6), 1364-1389.

Healy, G.N., Dunstan, D.W., Salmon, J., Cerin, E., Shaw, J., Zimmet, P.Z., \& Owen, N. (2008). Breaks in sedentary time: Beneficial associations with metabolic risk. Diabetes Care, 31(4), 661-666.

Healy, G.N., Matthews, C.E., Dunstan, D.W., Winkler, E.A.H., \& Owen, N. (2011). Sedentary itme and cardio-metabolic biomarkers in US adults: NHANES 200306. European Heart Journal, 32(5), 590-597.

Heraclides, A., Chandola, T., Witte, D.R., \& Brunner, E.J. (2009). Psychosocial stress at work doubles the risk of type 2 diabetes in middle-aged women: Evidence from the Whitehall II Study. Diabetes care, 32(12), 2230-2235.

Hernandez, L.M., \& Blazer, D.G. (2008). Genes, behavior, and the social environment: Moving beyond the nature/nurture debate. Institute of Medicine. Retrieved from, http://www.nap.edu/catalog/11693.html

Honjo, K. (2014). Social epidemiology: Definition, history, and research examples. Environmental Health and Preventive Medicine, 9(5), 193-199.

Hosmer, D.W., \& Lemeshow, S. (2000). Applied logistic regression. [2 ${ }^{\text {nd }}$ Ed.] John Wiley and Sons: Hoboken, NJ. doi: 10.1002/0471722146.fmatter

Howard, A.A., Arnsten, J.H., \& Gourevitch, M.N. (2004). Effect of alcohol consumption on diabetes mellitus: A systematic review. Annals of Internal Medicine, 140(3), 211-219. 
Hutchinson, E.D. (2011). Dimensions of human behavior: Person and environment. (4 ${ }^{\text {th }}$ Ed.). Sage Publications, Inc.: US.

Hwang, W.J.(2011). Philosophical and ethical perspectives on cardiovascular disease risk in low-wage workers. Public Health Nursing, 28(2), 168-175.

Ivengar, S. \& Chua, R.Y.J. (2011). Perceiving freedom givers: Effects of granting decision latitude on personality and leadership perceptions. Leadership Quaterly, 22(5), 863-880.

Jennings, J.R., Muldoon, M.F., Hall, M., Buyesse, D.J., \& Manuck, S.B. (2007). Selfreported sleep quality is associated with the metabolic syndrome. Sleep, 30(2), 219-223.

Jensen, C.C., Cnop, M., Hull, R.L., Fujimoto, W.Y., Kahn, S.E., \& The American Diabetes Association GENNID Study Group. (2002). $\beta$-cell function is a major contributor to oral glucose tolerance in high-risk relatives of four ethnic groups in the U.S. Diabetes, 51(7), 2170-2178.

Kahn, R. Buse, J., Ferranini, E., \& Stern, M. (2005). The metabolic syndrome: Time for a critical appraisal. Diabetes Care, 28(9), 2289-2304.

Kahn, B.B., \& Flier, J.S. (2000). Obesity and insulin resistance. The Journal of Clinical Investigations, 106(4), 473-481.

Kanuachi, M., Kanuachi, K., Inoue, T. Kimura, K., Saito, Y. (2007). Surrogate markers of insulin resistance in assessing individuals with new categories "prehypertension" and "prediabetes". Clinical Chemical Laboratory Medicine, 45(1), 35-39.

Karasek, R., \& Theorell, T. (1990). Healthy work: Stress, productivity, and the reconstruction of working life. Basic Books: New York, NY.

Karasek, R., Baker, D. Marxer, F., Ahlbom, A., \& Theorell, T. (1981). Job decision latitude, job demands, and cardiovascular disease: A prospective study of Swedish men. American Journal of Public Health, 71(7), 694-705.

Karasek, R., Brisson, C., Kawakami, N., Houtman, I., Bongers., P., \& Amik, B. (1998). The Job Content Questionnaire (JCQ): An instrument for internationally comparative assessments of psychosocial job characteristics. Journal of Occupational Health Psychology, 3(4), 322-355.

Karasek, R., Collins, S., Clays, E., Bortkeiwicz, A., \& Ferrario, M. (2010). Description of a large-scale design to assess work-stress-disease associations for cardiovascular disease. International Journal of Occupational Medicine and Environmental Health, 23(3), 293-312.

Karasek, R., Gardell, B., \& Lindell, J. (1987). Work and non-work correlates of illness and behavior in male and female Swedish white-collar workers. Journal of Occupational Behavior, 8(3), 187-207. 
Karasek, R., Pieper, C., \& Schwartz, J. (1985). Job Content Questionnaire and User's Guide. Job/Heart Project, Colombia University, New York.

Karlsson, B.H., Knutsson, A.K., Lindahl, B.O., \& Alfredsson, L.S. (2003). Metabolic disturbances in male workers with rotating three-shift work. Results of the WOLF study. International Archives of Occupational and Environmental Health, 76(6), 424-430.

Kenny, D.A. (2015). Measuring model fit. Retrieved from, http://davidakenny.net/cm/fit.htm

Kenny, D.A. (2015). Mediation. Retrieved from, http://davidakenny.net/cm/mediate.htm

Kessler, R.C., Berglund, P.A., Coulouvrat, C., Halak, G., Roth, T., Shahly, V., ...Walsh, J.K. (2011). Insomnia and the performance of US workers: Results from the American Insomnia Survey. Sleep, 34(9), 1161-1171.

Kivimaki, M., Leino-Arjas, P., Luukkonen, R., Riihimaki, H., Vahtera, J., \& Kirjonen, J. (2002). Work stress and risk of cardiovascular mortality: Prospective cohort study of industrial employees. British Medical Journal, 325(7369), 857-860.

Knudsen, H.K., Ducharme, L.J., \& Roman, P. M. (2007). Job stress and poor sleep quality: Data from an American sample of full-time workers. Social Science and Medicine, 64(10), 1997-2007.

Knutson, K.L., Ryden, A.M., Mander, B.A., \& Van Cauter, E. (2006). Role of sleep duration and quality in the risk and severity of Type 2 Diabetes Mellitus. Archives of Internal Medicine, 166(16), 1768-1774.

Knutson, K.L., Spiegel, K., Peney, P., \& Van Cauter, E. (2007). The metabolic consequences of sleep deprivation. Sleep Medicine Reviews, 11(3), 163-178.

Knutson, K.L., Van Cauter, E., Rathouz, P.J., Yan, L.L., Hulley, S.B., Liu, K., \& Lauderdale, D.S. (2009). Association between sleep and blood pressure in midlife: the CARDIA sleep study. Archives of Internal Medicine, 169(11), 10551061.

Kompier, M.A.J., Taris, T.W., \& vanVeldhoven, M. (2012). Tossing and turninginsomnia in relation to occupational stress, rumination, fatigue, and well-being. Scandinavian Journal of Work, Environment, \& Health, 38(3), 238-246.

Koppes, L.L.J., Dekker, J.M., Hendriks, H.F.J., Bouter, L.M., \& Heine, R.J. (2005). Moderate alcohol consumption lowers the risk of type 2 diabetes: A meta-analysis of prospective observational studies. Diabetes Care, 28(3), 710-725.

Kouvonen, A., Kivimaki, M., Elovainio, M., Virtanen, M., Linna, A., \& Vahtera, J. (2006). Job strain and leisure-time physical activity in female and male public sector employees. Preventive Medicine, 41(2), 532-539. 
Kouvonen, A., Kivimaki, M., Vaananen, A., Heponeimi, T., Elovainio, M., Ala-Mursula, L.,... Vahtera, J. (2007). Job strain and adverse health behaviors: The Finnish Public Sector Study. Journal of Occupational and Environmental Medicine, 49(1), 68-74.

Kouvonen, A., Vahtera, J., Oksanen, T., Pentti, J., Vaananen, A.K.P., Heponlemi, T., ...Kivimaki, M. (2012). Chronic workplace stress and insufficient physical activity: A cohort study. Occupational and Environmental Medicine, 7(1), 3-8.

Kumarasamy, S., Gopalakrishnan, K., Kim, D.H., Abraham, N.G., Johnson, W.D., Joe, B., \& Gupta, A.K. (2011). Dysglycemia induces abnormal circadian blood pressure variability. Cardiovascular Diabetology, 10(104). doi: 10.1186/14752840-10-104

Kunz-Ebrecht, S.R., Kirschbaum, C., \& Steptoe, A. (2003). Work stress, socioeconomic status and neuroendocrine activation over the working day. Social Science and Medicine, 58(8), 1523-1530.

Kuper, H., \& Marmot, M. (2003). Job strain, job demands, decision latitude, and risk of coronary heart disease within the Whitehall II Study. Journal of Epidemiology and Community Health, 57(2), 147-153.

Kutlu, M., Sonmez, A., Gene, H., Erdem, G., Tapan, S., Celebi, G., ...Erbil, M.K. (2009). Relationship between hemoglobin and CD40 ligand in prediabetes. Clinical Investigative Medicine, 37(6), E244-E250.

Landsbergis, P.A., Schnall, P.L., Pickering, T.G., Warren, K., \& Schwartz, J.E. (2003). Lower socioeconomic status among men in relation to the association between job strain and blood pressure. Scandinavian Journal of Work, Environment, and Health, 29(3), 206-215.

Landsbergis, P.A., Schnall, P.L., Belkic, K.L., Schwartz, J.E., Baker, D., \& Pickering, T.G. (2008). Work conditions and masked (hidden) hypertension - Insights into the global epidemic of hypertension. Scandinavian Journal of Work, Environment \& Health, Suppl(6), 41-51.

Lee, J.H., Hwang, S.Y., Kim, E.J., \& Kim, M.J. (2006). Comparison of risk factors between prehypertension and hypertension in Korean male industrial workers. Public Health Nursing, 23(4), 314-323.

Leka, S., Griffiths, A., \& Cox, T. (2004). Protecting workers health series no. 3. Work organization \& stress: Systematic problem approaches for employers managers and trade union representatives. World Health Organization. WHO Press: Geneva, Switzerland.

Leka, S., \& Jain, A. (2010). Health impact of psychosocial hazards at work: An Overview. World Health Organization. WHO Press: Geneva, Switzerland. 
Levi, J., Segal, L.M., St. Laurent, S., \& Kohn, D. (2011). F is for fat: How obesity threatens America's future. Robert Woods Johnson Foundation Report. Retrieved from, http://www.rwjf.org/content/dam/farm/reports/reports/2013/rwjf407528

Li, J., Jarczok, M.N., Loerbroks, A., Schollgen, I., Siegrist, J., Bosch, J., ...Fischer, J.E. (2013). Work stress is associated with diabetes and prediabetes: Cross-sectional results from the MIPH Industrial Cohort Studies. International Journal of Behavior and Medicine, 20(4), 495-503.

Lloyd, C., Smith, J., \& Weinger, K. (2005).Stress and diabetes: A review of the links. Diabetes Spectrum, 18(2), 121-127.

Lopenen, M., Hublin, C., Kalimo, R., Manttari, M., \& Tenkanen, L. (2010). Joint effect of self-reported sleep problems and three components of the metabolic syndrome on risk of coronary heart disease. Journal of Psychosomatic Research, 68(2), 149158.

MacKinnon, D.P., Fairchild, A.J., \& Fritz, M.S. (2007). Mediation analysis. Annual Review of Psychology, 58, 593-614.

Malin, S.K., Solomon, T.P., Blaszeczak, A., Finnegan, S., Filion, J., \& Kirwan, J.P. (2013). Pancreatic $\beta$-cell function increases in a linear dose-response manner following exercise training in adults with prediabetes. American Journal of Endocrinology and Metabolism, 305(10), E1248-E1254.

Mattke, S., Liu, H., Caloyeras, J.P., Huang, C.Y., VanBusum, K.R., Khodyakov, D., \& Shier, V. (2013). Workplace wellness programs study: Final report. RAND Health, RAND Corporation: Arlington, VA.

Marmot, M.G., Rose, G., Shipley, M., \& Hamilton, P.J.S. (1978). Employment grade and coronary heart disease in British civil servants. Journal of Epidemiology and Community Health, 32(4), 244-249.

Mirowsky, J. \& Ross, C.E. (2007). Creative work and health. Journal of health and social behavior, 48(4), 385-403.

Morin, C.M., Belleville, G., Belanger, L., \& Ivers, H. (2011). The Insomnia Severity Index: Psychometric indicators to detect insomnia cases and evaluate treatment response. Sleep, 24(5), 601-608.

Munir, F., Khan, H.T.A., Yarker, J., Haslam, C., Long, H., Bains, M., \& Kalawsky, K. (2009). Self-management of health-behaviors among older and younger workers with chronic illness. Patient Education and Counseling, 77(1), 109-115.

Muriff, H.J., Rothman, R.L., Byrne, D.W., \& Syngall, S. (2004). The impact of family history of diabetes. Diabetes Care, 27(9), 2247-2248.

McCraty, R., Atkinson, M., \& Tomasino, D. (2003). Impact of a workplace stress reduction program on blood pressure and emotional health in hypertensive 
employees. The Jounral of Alternative and Complementary Medicine, 9(3), 355369.

McNaughton, S.A., Dunstan, D.W., Ball, K., Shaw, J., \& Crawford, D. (2009). Dietary quality is associated with diabetes and cardio-metabolic risk factors. Journal of Nutrition, 139(4), 739-742.

Nasr, G.\& Sliem, H. (2012). Silent myocardial ischemia in prediabetics in relation to insulin resistance. Journal of Cardiovascular Disease Research, 193), 116-121.

Nathan, D.M., Davidson, M.B., DeFronzo, R.A., Heine, R.J., Henry, R.R., Pratley, R., \& Zinman, B. (2007). Impaired fasting glucose and impaired glucose tolerance. Diabetes Care, 30(3). 753-758.

National Institute of Diabetes and Diseases of the Kidney. (2012). Prediabetes, Retrieved from, http://diabetes.niddk.nih.gov/dm/pubs/statistics/\#fast

National Institute on Alcohol Abuse and Alcoholism. (2004). Recommended alcohol questions. Retrieved from, http://www.niaaa.nih.gov/research/guidelines-andresources/recommended-alcohol-questions

National Institute on Alcohol Abuse and Alcoholism. (2014). Rethinking drinking: Alcohol and your health. Retrieved from, http://rethinkingdrinking.niaaa.nih.gov

National Institutes of Health. National Institute on Alcohol Abuse and Alcoholism. (2014). Alcohol stats and statistics. Retrieved from, http://www.niaaa.nih.gov/alcohol-health/overview-alcohol-consumption/alcoholfacts-and-statistics

National Institutes of Health. (2013). What is high blood pressure? Retrieved from, http://www.nhlbi.nih.gov/health/health-topics/topics/hbp/

National Institute for Occupational Safety and Health. (2014). Stress... at work. Retrieved from, http://www.cdc.gov/niosh/docs/99-101/

National Institute for Occupational Safety and Health. (2015). Total worker health. Retrieved from, http://www.cdc.gov/niosh/twh/totalhealth.html

Computer Company. (2011). Best practices in value-based benefits. Retrieved from, http://www.tbgh.org/documents/TBGH_compendium_NI.pdf

Ndjaboué, R., Brisson, C., Vézina, M., Blanchette, C., \& Bourbonnais, R. (2014). Effort-reward imbalance and medically certified absence for mental health problems: A prospective study of white-collar workers. Occupational and Environmental Medicine, 71(1), 40-47. doi: 10.1136/oemed-2013-101375

Nedeltcheva, A.F., Kessler, L., Imperial, J., \& Penev, P.D. (2009). Exposure to recurrent sleep restriction in the setting of high caloric intake and physical inactivity results in increased insulin resistance and reduced glucose tolerance. Journal of Clinical Endocrinology and Metabolism, 94(9), 3242-3250. 
Neeland, I. J., Turner, A. T., Ayers, C.R., Powell-Wiley, T. M., Vega, G.L., FarzanehFar, R., ...Lemos, J.A. (2012). Dysfunctional adiposity and the risk of prediabetes and type 2 diabetes in obese adults. Journal of the American Medical Association, 308(11), 1150-1159.

Nishitani, N., Sakakibara, H., \& Akiyama, I. (2009). Eating behavior related to obesity and job stress in male Japanese workers. Nutrition, 25(1), 45-50.

Nunnally, J.C., \& Bernstein, I.H. (1994). Psychometric theory. $3^{\text {rd }}$ Ed. McGraw-Hill: United States.

Office of the Legislative Councel. (2010). Compilation of patient protection and affordable care act. United States House of Representatives.

Payne, N., Jones, F., \& Harris, P.R. (2005). The impact of job strain on the predictive validity of the Theory of Planned Behaviour: An investigation of exercise and healthy eating. British Journal of Healthy Psychology, 10(Pt 1), 115-131.

Perreault, L., Kahn, S., E., Christoph, C.A., Knowler, W.C., Hamman, R.F., \& Diabetes Prevention Program Research Group. (2009). Regression from pre-diabetes to normal glucose regulation in the Diabetes Prevention Program. Diabetes Care, 32(9), 1583-1588.

Petersen, K.F., \& Shulman, G.I. (2006). Etiology of insulin resistance. American Journal of Medicine, 119(Suppl 1), S10-S16.

Player, M.S., Diaz, V.A., Mainous, A.G., Gregorie, S.H., Knoll, M.E., \& Everett, C.J. (2011). Ethnic differences in the relationship of prediabetes with the presence of target-organ disease. Diabetes and Metabolism, 37(5), 403-409. doi: 10.1016/j.diab.2010.12.009

Preckel, D., vonKänel, R., Kudielka, B.M., \& Fischer, J.E. (2005). Overcommitment to work is associated with vital exhaustion. International Archives of Occupational \& Environmental Health, 78(2), 117-122.

Psaltopoulou, T., Ilias, I., \& Alevizaki, M. (2010). The role of diet and lifestyle in primary, secondary, and tertiary diabetes prevention: A review of meta-analyses. The Review of Diabetes Studies, 7(1), 26-35.

Punjabi, N.M., Shahar, E., Redline, S., Gottlieb, D.J., Givelber, R., \& Resnick, H.E. (2004). Sleep disordered breathing, glucose intolerance, and insulin resistance: The Sleep Heart Health Study. American Journal of Epidemiology, 160(6), 521630. doi: 10.1093/aje/kwh261

Pyykkönen, A-J, Räikkönen, K., Tuomi, T., Eriksson, J.G., Group, L, \& Isomaa, B. (2010). Stressful life events and the metabolic syndrome. Diabetes Care, 33(2), 378-384. doi: 10.2337/dc09-1027

Rafalson, L., Donahue, R., Stranges, S., LaMonte, M.J., Dmochowski, J., Dorn, J., \& Trevisan, M. (2010). Short sleep duration is associated with the development of 
impaired fasting glucose: The Western New York Health Study. American Epidemiology, 20.(12), 883-889

Rafferty, Y., Friend, R., \& Landsbergis, P.A. (2011). The association between job skill discretion, decision authority and burnout. Work and Stress, 15(1), 73-85.

Rao, S.S., Disraeli, P., \& McGregor, T. (2004). Impaired glucose tolerance and impaired fasting glucose. American Family Physician, 69(8), 1961-1968.

Reference. MD. (2013). Waist-hip ratio. Retrieved from, http://www.reference.md/files/D049/mD049629.html

Rhee, S.Y., Chon, S., Oh, S., Kim, S.W., Kim, J-W., Kim, Y.S., \& Woo, J. (2006). Insulin secretion and insulin resistance in newly diagnosed, drug naïve prediabetes and type 2 diabetes patients with/without metabolic syndrome. Diabetes Research and Clinical Practice, 76(3), 397-403.

Rolka, D.R., Li, Y., \& Geiss, L.S. (2008). Self-reported prediabetes and risk-reduction activities-United States, 2006. Morbidity and Mortality Weekly Report, 57(44), 1203-1205.

Rosmond, R., Dallman, M.F., \& Bjornthorp, P. (1998). Stress-related cortisol secretion in men: Relationships with abdominal obesity and endocrine, metabolic and hemodynamic abnormalities. Journal of Clinical Endocrinology and Metabolism, 83(6), 1853-1860.

Rosmond, R., Holm, G., \& Bjorntorp, P. (2000). Food-induced cortisol secretion in relation to anthropometric, metabolic, and haemodynamic variables in men. International Journal of Obesity and Related Metabolic Disorders, 24(4), 416422.

Ross, C.E., \& Mirowsky, J. (1999). Refining the association between education and health: The effects of quantity, credential, and selectivity. Demography, 36(4), 445-460.

Royston, P., \& Sauerberi, W. (2008). Multivariable model-building: A pragmatic approach to regression analysis based on fractional polynomials for modelling continuous variables. John Wiley \& Sons: Chichester, West Sussex, England.

Rubin, H.H., Witkiewitz, K., St. Andre, J., \& Reilly, S. (2007). Methods for handling missing data in the behavioral neurosciences: Don't throw the baby rat out with the bath water. The Journal of Undergraduate Neurosciences Education, 5(2), A71-A77.

Ryden, L., Stanl, E., Bartnik, M., Van den Berghe, G., Betteridge, J., de Boer, M-J, Cosentino, F., ...Thrainsdottir, I. (2007). Guidelines on diabetes, pre-diabetes, and cardiovascular diseases: Executive summary. European Heart Journal, 28(1), 88-136. 
Rydstedt, L.W., \& Lundh, M. (2010). An ocean of stress? The relationship between psychosocial workload and mental strain among engine officers in Swedish merchant fleet. International Maritime Health, 61(3), 168-175.

Saydah, S.H., Loria, C., Berhardt, M.S., \& Brancati, F.L. (2001). Subclinical states of glucose intolerance and risk of death in the U.S. Diabetes Care 24(3), 447-453.

Saltevo, J., Kautiainen, H., \& Vanhala, M. (2009). Gender differences in adiponectin and low-grade inflammation among individuals with normal glucose tolerance, prediabetes, and Type 2 diabetes. Gender Medicine, 6(3), 463-470. doi:10.1016/j.genm.2009.09.006

Santaguida, P.L., Balion, C., Hunt, D., Morrison, K., Gerstein, H., Raina, P., ...Yazdi, H. (2005). Diagnosis, prognosis, and treatment of impaired glucose tolerance and impaired fasting glucose. Evidence Report/Technology Assessment for the Agency for Healthcare and Research Quality. McMaster University: Ontario, Canada.

Sauter, S.L., Brightwell, W.S., Colligan, M.J., Hurrell, Jr., J.J., Katz, T.M., LeGrande, D.E., ...Tetrick, L.E. (2002). The changing organization of work and safety and health of working people. National Institute of Occupational Safety and Health Publication No. 2002-116.

Sauter, S.L, Colligan, M., Swanson, N., Hurrell, Jr., J., Scharf, Jr., F., Sinclair, R., ...Tisdale, J. (1999). Stress... at work. National Institute of Occupational Safety and Health Publication No. 99-101.

Schmidt, B., Bosch, J.A., Jarczok, M.N., Herr, R.M., Loerbroks, A., vanVianen, A.E.M., \& Fischer, J.E. (2015). Effort-reward imbalance is associated with the metabolic syndrome-Findings from the Mannheim Industrial Cohort Study (MICS). International Journal of Cardiology, 178, 24-28. doi: 10.1016/j.ijcard.2014.10.115

Schnall, P.L., Dobson, M., \& Rosskam, E. (2009). Unhealthy work: Causes, consequences, cures. Baywood Publishing Company: NY.

Schnall, P.L., Lansbergis, P.A., \& Baker, D. (1994). Job strain and cardiovascular disease. Annual Review of Public Health, 15, 381-411.

Segal-Isaacson, C.J., Wylie-Rosett, J., Gans, K.M. (2004). Validation of a short dietary assessment questionnaire: The Rapid Eating and Activity Assessment for Participants Short Version (REAP-S). The Diabetes Educator, 30(5), 774-781.

Sembajwe, G., Wahrendorf, M., Seigrist, J., Sitta, R., Zins, M., Goldberg, M., \& Berkman, L. (2012). Effects of job strain on fatigue: Cross-sectional and prospective views of the Job Content Questionnaire and Effort-Reward Imbalance in the GAZEL cohort. Occupational \& Environmental Medicine, 69(6), 377-384. doi: 10.1136/oem.2010.063503 
Siegrist, J. (1994). Emotions and health in occupational life: New scientific findings and policy implications. Patient Education and Counseling, 25(3), 227-236.

Siegrist, J. (1996). Adverse health effects of high-effot/low-reward conditions. Journal of Occupational Health Psychology, 1(1), 27-41.

Siegrist, J. (2012). Effort-reward imbalance at work - Theory, measurement and evidence. Department of Medical Sociology, University of Dusseldorf, Germany. Retrieved from, http://www.uniklinikduesseldorf.de/fileadmin/Datenpool/einrichtungen/institut_fuer_medizinische_soz iologie_id54/ERI/ERI-Website.pdf

Siegrist, J., Li, J., Montano, D. (2014). Psychometric properties of the Effort-Reward Imbalance Questinnaire. Department of Medical Sociology, University of Dusseldorf, Germany. Retrieved from, http://www.uniklinikduesseldorf.de/fileadmin/Datenpool/einrichtungen/institut fuer medizinische soz iologie_id54/ERI/PsychometricProperties.pdf

Seigrist, J., Peter, R., Cremer, P., \& Seidel, D. (1997). Chronic work stress is associated with atherogenic lipids and elevated fibrinogen in middle-aged men. Journal of Internal Medicine, 242(2), 149-156.

Siegrist, J., Stark, D. Chandola, T., Godin, I., Marmot, M., Neidhammer, I., \& Peter, R. (2004). The measurement of effort-reward imbalance at work: European comparisons. Social Science and Medicine, 58(8), 1483-1499.

Shetty, G.K, Economides, P.A., Horton, E.S., Mantzoros, C.S., \& Veves, A. (2004). Circulating adiponectin and resistin levels in relation to metabolic factors, inflammatory markers, and vascular reactivity in diabetic patients and subjects at risk for diabetes. Diabetes Care, 27(10), 2450-2457.

Silvertsen, B., Overland, S., Neckelmann, D., Glozier, N., Krokstad, S., Pallesen, S., ...Mykletun, S. (2006). The long-term effect of insomnia on work disability: The HUNT-2 Historical Cohort Study. American Journal of Epidemiology, 163(11), 1018-1022. doi: 10.1093/aje/kwj145

Skalamera, J., \& Hummer, R.A. (2016). Educational attainment and the clustering of health-related behavior among U.S. young adults. Preventive Medicine, 84, 83-89. doi: 10.1016/j.ypmed.2015.12.011. Epub 2015 Dec 29

Solomon, C.G. (1999). The epidemiology of polycystic ovarian syndrome. Prevalence and associated disease risks. Endocrinology and Metabolism Clinics of North America, 28(2), 247-263.

Spiegel, K., Leproult, R., \& van Carter, E. (1999). Impact of sleep debt on metabolic an endocrine function. The Lancet, 354(9188), 1435-1439.

Srikanthan, P., \& Karlamangla, A.S., (2011). Relative muscle mass is inversely associated with insulin resistance and prediabetes. Findings from the Third 
National Health and Nutrition Examination Survey. Journal of Clinical Endocrinology and Metabolism, 96(9), 2898-2903.

Su, Y., Liu, X.-M., Sun, Y.-M., Jin, H.-B., Fu, R., Wang, Y.-Y., \& Luan, Y. (2008). The relationship between endothelial dysfunction and oxidative stress in diabetes and prediabetes. International Journal of Clinical Practice, 62(6), 877-882. doi: 10.1111/j.1742-1241.2008.01776.x

Surwit, R.S., \& Schneider, M.S. (1993). Role of stress in the etiology and treatment of diabetes mellitus. Psychosomatic Medicine, 55(4), 380-393..

Suwazono, Y., Dochi, M., Oishi, M., Tanaka, K., Kobayashi, E., \& Sakata, K. (2009). Shiftwork and impaired glucose metabolism: A 14-year cohort study on 7104 male workers. Chronobiology International, 26(5), 926-941.

Szoslang, D. (2010). Shiftwork and metabolic syndrome, diabetes mellitus, and ischaemic heart disease. International Journal of Occupational Medicine and Environmental Health, 23(3), 287-291.

Taris, T.W., Ybema, J.F., Beckers., D.G.J., Verheijden, M.W., Geurts, S.A.E., \& Kompier, M.A.J. (2011). Investigating the associations among overtime work, health behaviors, and health: A longitudinal study among full-time employees. International Journal of Behavioral Medicine, 18(4), 352-360.

Taylor, L.M., Spence, J.C., Raine, K., Plotnikoff, R.C., Vallance, J.K., \& Sharma, A.M. (2010). Physical activity and health-related quality of life in individuals with prediabetes. Diabetes Research and Clinical Practice, 90(1), 15-21. doi:10.1016/j.diabres.2010.04.011

The Diabetes Prevention Program Research Group. (2002). The diabetes prevention program: Description of lifestyle intervention. Diabetes Care, 25(12), 2165-2171

Toshihiro, M., Saito, K., Takikawa, S., Takebe, N., Onoda, T., \& Satoh, J. (2008). Psychosocial factors are independent risk factors for the development of Type 2 diabetes in Japanese workers with impaired fasting glucose and/or impaired glucose tolerance. Diabetic Medicine, 25(10), 1211-1217.

Trudel, L., Vonarx, N., Simard, C., Freeman, A., Vézina, M., Brisson, C, ...Dugas, N. (2009). The adverse effects of psychosocial constraints at work: A participatory study to orient prevention to mitigate psychological distress. Work, 34(3), 345347. doi: 10.3233/WOR-2009-0933

United States Bureau of Labor and Statistics. Division of Labor Force Statistics. (2014). Labor force statistics from the Current Population Survey, Retrieved from, http://www.bls.gov/cps/industry age.htm

United States Department of Health and Human Services. National Diabetes Information Clearinghouse. (2011). National diabetes statistics, 2011. Retrieved from, http://diabetes.niddk.nih.gov/dm/pubs/statistics/\#Gestational 
United States Department of Labor. Bureau of Labor and Statistics. (2014). Employment projections: Classification and crosswalks, Retrieved from, http://www.bls.gov/emp/ep_crosswalks.htm

United States Department of Labor. Bureau of Labor and Statistics. (2014). Employment projections: Education and training data, Retrieved from, http://www.bls.gov/emp/ep_education_training_system.htm

United States Department of Labor. Bureau of Labor and Statistics. (2012). Labor force statistics from the Current Population Survey. Occupational and industry classification systems used in the Current Population Survey. Retrieved from, http://www.bls.gov/cps/cpsoccind.htm

United States House of Representatives. Office of the Legislative Counsel. (2010). Compilation of patient protection and affordable care act. Eleventh Congress, $2^{\text {nd }}$ session: Washington, DC.

Urbanavicius, V., Abraitiene, A., Vitkus, D., Borovkiene, R., \& Duciniskiene, Z.A. (2008). Adiponectin and uric acid in pre-diabetes and early type 2 diabetes mellitus. Acta Medica Lituanica, 15(3), 81-87.

Vaananen, A., Anttila, E., Turtiainen, J., \& Varje, P. (2012). Formulation of work stress in 1960-2000: Analysis of scientific works from the perspective of historical sociology. Social Science and Medicine, 75(5), 784-794.

van der Doef, M., \& Maes, S. (1999). The Job Demand-Control (-Support) Model and psychological well-being: A review of 20 years of empirical research. Work and Stress, 13(2), 87-114.

Vrijkotte, T.G.M., vonDoornen, L.J.P., deGues, E.J.C. (2000). Effects of work stress on ambulatory blood pressure, heart rate, and heart rate variability. Hypertension, 35, 880-886. doi: 10.1161/01.HYP.35.4.880

Wellen, K.E., \& Hotamisligil, G.S. (2005). Inflammation, stress, and diabetes. Journal of Clinical Investigation, 115(5), 1111-1119. doi:10.1172/JCI25102

Williams, E.D., Magliano, D.J, Tapp, R.J., Oldenbrug, B.F., \& Shaw, N.E. (2013). Psychosocial stress predicts abnormal glucose metabolism: The Australian Diabetes, Obesity, and Lifestyle (AusDiab) Study. Annals of Behavioral Medicine, 46(1), 62=72.

Taris, T.W., Ybema, J.F., Beckers, D.G.J., Verheijden, M.W., Geurts, S.A.E, \& Kompier, M.A.J. (2011). Investigating the associations among overtime work health behaviors, and health: A longitudinal study among full-time employees. International Journal of Behavior and Medicine, 18(4), 352-360.

United States Department of Health and Human Services. (2012). Test procedure for smoking status. Office of the National Coordinator for Health Information Technology.

Retrieved from, 
http://www.healthit.gov/sites/default/files/170.314a11smokingstatus 2014 tp ap provedv1.2.pdf

United States Department of Health and Human Services. (2014). The health consequences of smoking - 50 years of progress: A report of the Surgeon General. Retrieved from, http://www.surgeongeneral.gov/library/reports/50-yearsof-progress/full-report.pdf

Vedhara, K., \& Irwin, M. (2007). Human psychoneuroimmunology. Oxford University Press: New York, NY.

vonBonsdorff, M.B., Seitsamo, J., vonBonsdorff, M.E., Ilmarinen, J., Nygärd, C-H., \& Rantanen, T. (2012). Job strain among blue-collar and white-collar employees as a determinant of total mortality: A 28-year population-based follow-up. British Medical Journal Open Access, 2(2), e000860. doi: 10.1136/bmjopen-2012000860

Wagner, R. Thorand, B., Osterhoff, M.A., Muller, G., Bohm, A., Meisinger, C., ...Fritsche, A. (2013). Family history of diabetes is associated with higher risk for prediabetes: A multicentre analysis from the German Center for Diabetes Research. Diabetologia, 56(10), 2176-2180.

The White House. Office of Management and Budget. (1995). Standards for the classification of race and ethnicity. Retrived from, http://www.whitehouse.gov/omb/fedreg_race-ethnicity

Wolk, R., \& Somers, V.K. (2006). Sleep and the metabolic syndrome. Experimental physiology, 92(1), 67-78.

World Health Organization. (2007). Workers' health: Global plan of Action. Sixtieth World Health Assembly: World Health Organization.

Wu, M-C., Yan, Y-C., Wu, J-S., Wang, R-H., Lu, F-H., \& Chang, C-J. (2012). Short sleep duration is associated with a higher prevalence of metabolic syndrome in an apparently healthy population. Preventive Medicine, 55(4), 305-309.

Xu, W., Hang, J., Gao, W., Zhao, Y., Li, W., Wang, X., ...Guo, L. (2012). Association between effort-reward imbalance and glycosylated hemoglobin (HbA1c) among Chinese workers: Results from SHISO study. International Archives of Occupational and Environmental Health, 85(2), 215-220. doi: 10.1007/s00420011-0650-7

Yoo, H., \& Franke, W.D. (2011). Stress and cardiovascular disease risk in female law enforcement officers. International Archives of Occupational and Environmental Health, 84(3), 279-286. doi: 10.1007/s00420-010-0548-9

Zhang, Y., Dall, T.M., Chen, Y., Baldwin, A., Yang, W., Mann, S., Moore, V., LeNestour, W., \& Quick, W.W. (2009). Medical cost associated with prediabetes. Population Health Management, 1293), 157-163. doi: 10.1089/pop.2009.12302 


\section{Vita}

Raquel Yvonne Reynolds was born in Laredo, Texas. After completing her work at St. Augustine Catholic High School, Laredo, Texas, in 1985, Raquel entered St. Edward's University. After two years at St. Edward's University, she transferred to Murray State College, Tishomingo, Oklahoma where she completed her Associate Degree in Nursing in 1991. During the next 10 years, she worked as a registered nurse. In spring of 2001, she entered the RN to BSN program The University of Texas at Austin and completed her Bachelor of Science in Nursing (BSN) degree in 2003. Immediately after completing her BSN, Raquel entered graduate school at The University of Texas at Austin, Master of Science in Nursing (MSN) program. She completed her MSN with a public health nursing concentration in 2005. In fall of 2009, she started the doctoral program at the University of Texas at Arlington. She transferred to The University of Texas School of Nursing doctoral program fall of 2010.

Address: rreynolds@utexas.edu

This manuscript was typed by the author. 